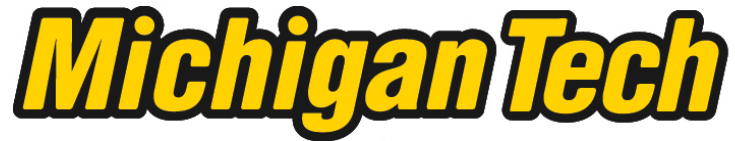 \\ Michigan Technological University Create the Future Digital Commons @ Michigan Tech
}

Dissertations, Master's Theses and Master's Reports - Open

Dissertations, Master's Theses and Master's

Reports

2010

\section{Modeling mechanical response of heterogeneous materials}

Siladitya Pal

Michigan Technological University

Follow this and additional works at: https://digitalcommons.mtu.edu/etds

Part of the Mechanical Engineering Commons

Copyright 2010 Siladitya Pal

\section{Recommended Citation}

Pal, Siladitya, "Modeling mechanical response of heterogeneous materials", Dissertation, Michigan Technological University, 2010.

https://doi.org/10.37099/mtu.dc.etds/394

Follow this and additional works at: https://digitalcommons.mtu.edu/etds

Part of the Mechanical Engineering Commons 
MODELING MECHANICAL RESPONSE OF HETEROGENEOUS MATERIALS

By

SILADITYA PAL

\author{
A DISSERTATION \\ Submitted in partial fulfillment of the requirements \\ for the degree of \\ DOCTOR OF PHILOSOPHY \\ (Mechanical Engineering-Engineering Mechanics) \\ MICHIGAN TECHNOLOGICAL UNIVERSITY
}

2010

(C) 2010 Siladitya Pal 

This dissertation, "Modeling mechanical response of heterogeneous materials", is hereby approved in partial fulfillment of the requirements for the degree of DOCTOR OF PHILOSOPHY in the field of Mechanical Engineering-Engineering Mechanics.

\section{DEPARTMENT:}

Mechanical Engineering-Engineering Mechanics

\section{Signatures:}

Dissertation Advisor

Dr. Spandan Maiti

Committee

Dr. Gregory M. Odegard

Dr. Tammy L. Haut Donahue

Dr. Yue Li

Department Chair

Dr. William W. Predebon

Date 

To my family 


\title{
Contents
}

\author{
List of Figures
}

xiii

List of Tables

XXV

List of Algorithms

xxvii

Acknowledgments

xxix

Abstract

xxxi

1 Introduction

1.1 Heterogeneous materials and their technological importance . . . . 1.1.1 Cellular materials . . . . . . . . . . . . . 3

1.1.2 Structural biomimetic materials ............. 4

1.2 Computational mechanics applied to the deformation and failure response of heterogeneous materials . . . . . . . . . . . . .

1.2.1 Literature review .................. . . . 6

1.2.1.1 Dynamic response of cellular materials . . . . . . 6

1.2.1.2 Mechanical behavior of mussel byssus thread . . . . 10

1.2.1.3 Computational complexity for the simulation of heterogeneous materials response . . . . . . . . . 13

1.2.2 Technological challenges . . . . . . . . . . . . . 16 
1.2.2.1 Computational frameworks for cellular and biological materials ................ . . 16

1.2.2.2 Efficient predictive tools for complex materials . . . . 18

1.3 Research objectives . . . . . . . . . . . . . . . . . 19

1.4 Scope of the present study \& thesis organization . . . . . . . . . . . . 21

2 Development of computational framework $\quad 23$

2.1 Corotational beam formulation .............. 24

2.1.1 Beam kinematics .................. 24

2.1.1.1 Earlier scheme and its limitation . . . . . . . 25

2.1.1.2 Proposed scheme for kinematic quantities . . . . . 27

2.1.2 Calculation of virtual internal work . . . . . . . . . . 28

2.1.3 Euler Bernoulli beam element . . . . . . . . . . . . . 30

2.1.4 Timoshenko beam element . . . . . . . . . . . . . . 31

2.1.5 Calculation of axial force and moments . . . . . . . . . . . 32

2.2 Explicit transient finite elements for the beam element . . . . . . . 33

2.3 Implicit quasi-static finite element corotational beam formulation . . 35

2.4 Simulation for the verification of developed computational scheme. . . 37

2.4.1 Deflection of elastic cantilever beam under impact load: Theory and numerical experiment ............... 37

2.4.2 Deformation of a crossed bar under impact load: demonstration of dynamic buckling . . . . . . . . . . . . . . 38

2.5 Cellular microstructure generation \& randomness quantification . . . 40

2.5.1 Voronoi tessellation . . . . . . . . . . . . . . 41

2.5.2 Numerical implementation of cellular microstructure generation 41

2.6 Experimental validation of foam dynamic response . . . . . . . 43

2.7 Concluding remarks . . . . . . . . . . . . . . . . . . . 44

3 Analysis of stress state in soft cellular materials during split Hop- 
\begin{tabular}{ll} 
kinson pressure bar (SHPB) test & 47 \\
\hline
\end{tabular}

3.1 Introduction . . . . . . . . . . . . . . . 47

3.2 Problem description . . . . . . . . . . . . . . . . . . 48

3.2.1 Setup of the computational model . . . . . . . . . . . 48

3.2.2 Extraction of dynamic stress-strain response . . . . . . . . 50

3.2.3 Theoretical verification . . . . . . . . . . . . . 51

3.2.4 A typical stress equilibration study . . . . . . . . . . 53

3.2.5 Intrinsic parameters of the model . . . . . . . . . . . 54

3.3 Result and discussions . . . . . . . . . . . . . . . 57

3.3.1 Effect of cell size $d_{a v}$ and number of cells $\bar{\kappa}$ in the specimen length 58

3.3.2 Effect of loading rate . . . . . . . . . . . . . . . 67

3.3.3 Effect of end-impedance mismatch ratio . . . . . . . . 71

3.4 Concluding remarks . . . . . . . . . . . . . . . . . . 72

4 Microscopic deformation mechanisms associated with the transient response of soft cellular materials* $\quad 75$

4.1 Introduction . . . . . . . . . . . . . . . . . 75

4.2 Problem description . . . . . . . . . . . . . . . . 76

4.3 Result and discussions . . . . . . . . . . . . . . . . 79

4.3.1 Effect of pulse rise time . . . . . . . . . . . 82

4.3.2 Effect of cell size . . . . . . . . . . . . 87

4.3.3 Effect of material properties ............. 92

4.3.3.1 Effect of Young's modulus . . . . . . . . . . 92

4.3.3.2 Effect of bulk material density . . . . . . . . 95

4.3.4 Effect of cell shape irregularities . . . . . . . . . . . . . 98

4.3.5 Effect of cell orientation . . . . . . . . . . 102

4.4 Concluding remarks . . . . . . . . . . . . . . . . . . . 104

5 Role of heterogeneity on the mechanical performance of byssus thread 107 
5.1 Introduction . . . . . . . . . . . . . . . . 107

5.2 Numerical framework . . . . . . . . . . . . . . . . . . . 108

5.2 .1 Mathematical model . . . . . . . . . . . . . . . 108

5.2 .2 Experimental validation $\ldots \ldots \ldots \ldots \ldots$

5.2 .3 Optimization formulation . . . . . . . . . . . 113

5.3 Result and discussions . . . . . . . . . . . . . . . . 115

5.3 .1 Identification of critical parameters . . . . . . . . . 119

5.3.2 Evaluation of optimal percentage of proximal thread length . . 123

5.4 Concluding remarks . . . . . . . . . . . . . . . . . 126

6 Surrogate modeling techniques applied to heterogeneous materials 129

6.1 Introduction . . . . . . . . . . . . . . . . . . . 129

6.2 Mathematical foundation of HDMR . . . . . . . . . . . . . 130

6.3 Application 1: Determination of failure envelope for heterogeneous materials $^{*} \ldots \ldots \ldots \ldots \ldots \ldots \ldots$

6.3.1 HDMR formalism for general feasibility problem . . . . . . 136

6.3.2 HDMR applied to failure envelop determination for a particulate composite system . . . . . . . . . . . . . . . 138

6.4 Application 2: Efficient multiscale analysis of nonlinear hyperelastic materials undergoing large deformation . . . . . . . . . . . 144

6.4.1 Surrogate model based multiscale framework . . . . . . . 145

6.4.1.1 Macroscale formulation . . . . . . . . . . . 145

6.4.1.2 Surrogate model of RVE using HDMR formulation . 148

6.4.1.3 Microscale formulation . . . . . . . . . . . . . 150

$6.4 .2 \quad$ Numerical examples . . . . . . . . . . . . . . . . . 152

6.4.2.1 Particulate composites . . . . . . . . . . . . . 152

6.4.2.2 Short fiber composite. . . . . . . . . . . . 158

6.4.2.3 Three dimensional particulate composite . . . . . . 160

6.4.3 Computational efficiency . . . . . . . . . . . . . . . . . . 163 
6.5 Concluding remarks . . . . . . . . . . . . . . . . 166

\begin{tabular}{lll}
7 & Conclusions and future work & 169 \\
\hline
\end{tabular}

7.1 Salient findings of the present work . . . . . . . . . . . . . 169

7.2 Future directions . . . . . . . . . . . . . . . . . . 173

A Cell level analysis of microinertia and microbuckling in soft cellular \begin{tabular}{l|l} 
materials & $\mathbf{1 7 7}$
\end{tabular}

A.1 Introduction . . . . . . . . . . . . . . . . 177

A.2 Analytical model . . . . . . . . . . . . . . . . . . 181

A.3 Result and discussions . . . . . . . . . . . . . . . . . . 188

A.3.1 Effect of impact velocity . . . . . . . . . . . . . 190

A.3.2 Effect of strut length . . . . . . . . . . . . 193

A.3.3 Effect of cell size irregularity . . . . . . . . . . . . 195

A.3.4 Effect of bulk material density . . . . . . . . . . . . . . 197

A.3.5 Effect of Young's modulus . . . . . . . . . . . . . 200

A.3.6 Effect of viscosity . . . . . . . . . . . . . 202

A.4 Concluding remarks . . . . . . . . . . . . . . . 203

$\begin{array}{ll}\text { B Voronoi cell generation for cellular microstructure } & 206\end{array}$

\begin{tabular}{ll} 
C Plasticity algorithm & 208 \\
\hline
\end{tabular}

C.1 Governing equations . . . . . . . . . . . . . 208

C.2 Numerical implementation . . . . . . . . . . . . . . . . . . 210

\begin{tabular}{ll} 
D Finite element formulation of large strain hyperelasticity & 213 \\
\hline
\end{tabular}

D.1 Linearization of equilibrium condition (Lagrangian formulation) . . . 213

D.2 Finite element implementation . . . . . . . . . . . . . 215

D.3 Newton-Raphson solution algorithm and line search method . . . . 216

D.4 Line search algorithm . . . . . . . . . . . . . . . 217 
E Genetic algorithm based optimization $\quad 219$

E.1 Optimization formulation . . . . . . . . . . . . 219

E.2 Genetic algorithm . . . . . . . . . . . . . . . 220

E.3 Numerical implementation . . . . . . . . . . . . . . . . . . . 221

F Measurement of cellular deformation $\quad 225$

\begin{tabular}{l|l} 
G Copyrights & 227 \\
\hline
\end{tabular} 


\section{List of Figures}

2.1 Schematic diagram of a 2D corotational beam element. $x-y$ is the global coordinate system while $x_{l}-y_{l}$ is the rotated local coordinate system in which local deformations are defined. . . . . . . . . . . . . .

2.2 Schematic diagram of a 2D corotational beam element undergoing large rotation. $x-y$ is the global coordinate system while $x_{l}-y_{l}$ is the rotated local coordinate system in which local deformations are defined. $x^{\prime \prime}-y^{\prime \prime}$ denotes local coordinate under large rotation. An intermediate configuration is denoted by $x_{l}^{\prime}-y_{l}^{\prime}$. . . . . . . . . . . . . . . .

2.3 Stress distribution across the depth of the beam when constitutive material is inelastic. . . . . . . . . . . . . . . . . . . . .

2.4 Cantilever beam subjected to transient force at the tip. . . . . . . . 38

2.5 (a) Variation of impact force at the tip with time, and (b) Variation of tip deflection with time. . . . . . . . . . . . 38

2.6 Schematic of crossed bar undergoing axial impact at the free end. . . 39

2.7 Deformed snapshots of the crossed bar when subjected to axial impact at the free end. . . . . . . . . . . . . . . .

2.8 Voronoi tessellation showing Voronoi sites, Voronoi regions and a Voronoi diagram. . . . . . . . . . . . . . . . . . 
2.9 (a) Numerically generated random open cellular structure with an average cell size $d_{a v}=28.4 \mu \mathrm{m}$ and standard deviation $S_{d}=0.02 d_{a v}$, and (b) resulting cell size distribution normalized with $d_{a v}$. Frequency of occurrence of a particular cell size is denoted by $N_{f}$, while $d_{c}$ stands for the size of an individual cell. . . . . . . . . . . . . . . . .

2.10 Comparison of simulated and experimental responses of a cellular spec-

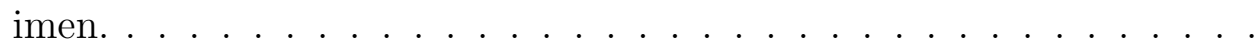

3.1 A cellular specimen of length $L\left(=13.2 d_{a v}\right)$ and depth $D$ is held between incident and transmission bars of a SHPB set up. Impedance (viscous) boundary conditions are used at left and right ends of the specimen while top and bottom boundaries are kept free. . . . . . . . . . .

3.2 Comparison of simulated stress equilibration curves for a ceramic specimen being tested in a SHPB setup with analytical results. . . . . . .

3.3 Typical stress equilibration curve $R(t)$ against normalized time $(\bar{t}=$ $\left.t / t_{0}\right)$ as obtained for a cellular material. The stress equilibration measure, as characterized by RMS value of the signal, is defined over the time period between $t_{c}$ and $t_{f}$. Inset shows the blow up of signal $R(t)$ over a time range of $t_{c}=20 t_{0}$ and $t_{f}=40 t_{0} \ldots \ldots \ldots$. . . . . .

3.4 Variation of $\bar{\eta}$ with respect to relative density of the specimen with $\bar{\kappa}=26.4$. Error bars represent the variation of $\bar{\eta}$ with respect to aspect ratio $\phi$ for each instance of the relative density $\bar{\rho}$. . . . . . . . . . .

3.5 Trapezoidal loading pulse with a velocity $v$ and rise time $t_{R}$. Final time $t_{f}$ corresponds to a time instant up to which the stress equilibration measure $R_{r m s}$ is computed. . . . . . . . . . . . . . .

3.6 Variation of relative density $\bar{\rho}$ with normalized average cell size $\bar{d}_{a v}$ and number of cells in the specimen length $\bar{\kappa}$ for a fixed strut thickness

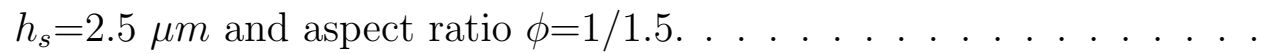


3.7 Variation of $R_{r m s}$ with number of cells along the specimen length $\bar{\kappa}$ for a range of normalized cell size $\bar{d}_{a v}$. Strut thickness $h_{s}$ is held constant of $2.5 \mu \mathrm{m}$ while aspect ratio $\phi=1 / 1.5$ for all the simulations. . . . .

3.8 Effect of aspect ratio $\phi$, represented by error bars, on $R_{r m s}$ (a) for a constant normalized average cell size $\left(\bar{d}_{a v}=15.1\right)$ but varying $\bar{\kappa}$, and (b) for a fixed number of cells $(\bar{\kappa}=26.4)$ but different $\bar{d}_{a v}$. . . . . . . . .

3.9 Stress-strain response of cellular specimens with a constant cell size $\left(\bar{d}_{a v}=15.1\right)$ but different number of normalized cells in the specimen length $(\bar{\kappa})$. Numbers in parentheses indicate actual number of layers of cells in the specimen length direction. . . . . . . . . . . . .

3.10 (a) Stress-strain curves for cellular specimen for the three cases designated in Table 3.1, and (b) corresponding stress equilibrium history. .

3.11 Snapshots of deformed specimens at selected time intervals for the three cases $(A, B$ and $C$ ) designated in Table 3.1. Degree of deformation in each cell is represented by a grey scale varying from 0 (no deformation) to 1 (full collapse). . . . . . . . . . . . . . . . . . .

3.12 RMS values of the stress equilibration measure $\left(R_{r m s}\right)$ as a function of relative density $\bar{\rho}$ and maximum applied velocity $\bar{v}$ with a constant rise time $\left(\bar{t}_{R}=25\right) \ldots \ldots \ldots \ldots \ldots \ldots \ldots \ldots \ldots \ldots \ldots \ldots \ldots \ldots \ldots \ldots$

3.13 (a) Stress-strain response, and (b) stress equilibration curves for the four cases chosen from Figure 3.12 . . . . . . . . . . . . . . . . .

3.14 Contour plot of $R_{r m s}$ for different relative density materials with $\bar{\kappa}=13.2$, fixed applied velocity $\bar{v}=0.01$, and $\bar{Z}=\bar{Z}_{I}=\bar{Z}_{T}=6$ subjected to loading pulses with different rise times $\bar{t}_{R}$. All values of $R_{r m s}$ shown are in (\%). 70

3.15 Contour plot of $R_{r m s}$ for different relative density materials subjected to different end impedance mismatch ratios $\bar{Z}=\bar{Z}_{I}=\bar{Z}_{T}$ with $\bar{\kappa}=13.2$, fixed applied loading $\left(\bar{v}=0.01\right.$ and $\left.\bar{t}_{R}=25\right)$. All values of $R_{r m s}$ shown are in $(\%) \ldots \ldots \ldots \ldots \ldots$ 
4.1 (a) A cellular specimen of length $L$ and depth $D$ is held against a rigid boundary. The specimen is subjected to an impact velocity $v_{a}$ denoted by arrows at the right end. (b) Applied velocity pulse with a magnitude $v_{a}$ and rise time $t_{R} \ldots \ldots \ldots \ldots$

4.2 Stress-strain response for two different collapse modes: uniform collapse indicated by solid line, and progressive collapse denoted by dashed line. Bulk density of the material is taken as $910 \mathrm{kgm}^{-3}$ for the first case, while that for the second case is $6825 \mathrm{kgm}^{-3}$. Young's modulus for both the materials are fixed at $0.6 \mathrm{GPa}$, while dimension of the specimen for both the cases are also held constant. . . . . . . . . .

4.3 Snapshots of deformed specimens for (a) progressive collapse and (b) uniform collapse. Degree of deformation in each cell is represented by a gray scale varying from 1 (no deformation) to 0 (full collapse). . . .

4.4 Stress-strain responses of a cellular material with different pulse rise times at (a) $\dot{\varepsilon}=1.0 \times 10^{5} s^{-1}$ and (b) $\dot{\varepsilon}=2.0 \times 10^{4} s^{-1} \ldots$. . . . . . . .

4.5 Plateau stress $\sigma_{p l}$ for different pulse rise times and strain rates. . . . .

4.6 Snapshots of deformed specimens at selected strain intervals for (a) $t_{R}=0.3 \mu \mathrm{s}$ and (b) $t_{R}=9.3 \mu \mathrm{s}$ at a strain rate $\dot{\varepsilon}=1.0 \times 10^{5} \mathrm{~s}^{-1}$. The cellular material is made of polyethylene with $d_{a v}=25.2 \mu \mathrm{m}$. Degree of deformation in each cell is represented by a gray scale varying from 1 (no deformation) to 0 (full collapse). . . . . . . . . . . . .

4.7 Stress-strain behavior of a cellular material with different cell sizes $d_{a v}$ and, equivalently, apparent densities $\rho$ at a strain rate $\dot{\varepsilon}=1.0 \times 10^{5} \mathrm{~s}^{-1}$

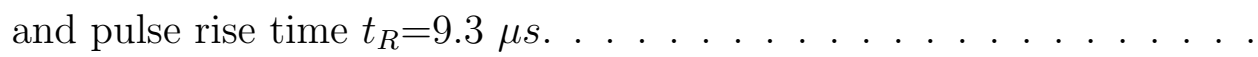

4.8 Plateau stress for cellular specimens with (a) cell sizes and (b) square of cell sizes when subjected to different strain rates. The pulse rise time is fixed at $t_{R}=9.3 \mu \mathrm{s} . \ldots \ldots \ldots$ 
4.9 Evolution of local strain $\varepsilon_{l}$ with time along specimen length for (a) $d_{a v}=37.5 \mu \mathrm{m}$ and (b) $d_{a v}=18.9 \mu \mathrm{m}$ at a strain rate $\dot{\varepsilon}=1.0 \times 10^{5} \mathrm{~s}^{-1}$ and $t_{R}=9.3 \mu \mathrm{s}$. The distance of any point along specimen length from the rigid boundary is denoted by $\xi$, where $\xi=0$ and $\xi=L$ specify support and impact ends, respectively. . . . . . . . . . . . . .

4.10 Snapshots of deformed specimens at selected time intervals for (a) $d_{a v}=37.5 \mu \mathrm{m}$ and (b) $d_{a v}=18.9 \mu \mathrm{m}$ at a strain rate $\dot{\varepsilon}=1.0 \times 10^{5} \mathrm{~s}^{-1}$ with $t_{R}=9.3 \mu \mathrm{s}$. Degree of deformation in each cell is represented by a gray scale varying from 1 (no deformation) to 0 (full collapse). . . . .

4.11 Stress-strain response of a cellular material for different Young's moduli at a strain rate $\dot{\varepsilon}=1.0 \times 10^{5} \mathrm{~s}^{-1}$ and for pulse rise time $t_{R}=9.3 \mu \mathrm{s}$. The cell size of the specimen is $d_{a v}=25.2 \mu \mathrm{m} . \ldots$. . . . . . . .

4.12 Plateau stress for cellular specimens with various Young's moduli when subjected to different strain rates. The pulse rise time is fixed at $t_{R}=9.3$ 

4.13 Snapshots of deformed specimens at selected time intervals for (a) $E=0.15 \mathrm{GPa}$ and (b) $E=1.5 \mathrm{GPa}$ at a strain rate $\dot{\varepsilon}=1.0 \times 10^{5} \mathrm{~s}^{-1}$ with $t_{R}=9.3 \mu \mathrm{s}$. Degree of deformation in each cell is represented by a gray scale varying from 1 (no deformation) to 0 (full collapse). The

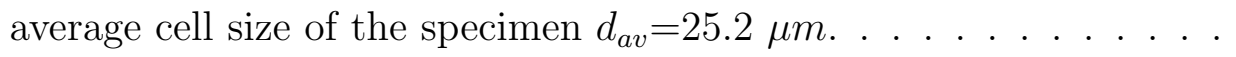

4.14 Stress strain response of a cellular material with different bulk material densities at a strain rate $\dot{\varepsilon}=1.0 \times 10^{5} \mathrm{~s}^{-1}$ and $t_{R}=9.3 \mu \mathrm{s}$. The cell size of the specimen given as $d_{a v}=25.2 \mu \mathrm{m} . \ldots$. . . . . . . . . . .

4.15 Evolution of local strain with time along specimen length for (a) $\rho_{s}=910$ $\mathrm{kgm}^{-3}$ and (b) $\rho_{s}=6825 \mathrm{kgm}^{-3}$ at a strain rate $\dot{\varepsilon}=1.0 \times 10^{5} \mathrm{~s}^{-1}$ and $t_{R}=9.3 \mu \mathrm{s}$. The distance of any point along specimen length from the support boundary is denoted by $\xi$, where $\xi=0$ and $\xi=L$ specify support and impact end respectively. . . . . . . . . . . . . . . 
4.16 Snapshots of deformed specimens at selected time intervals for (a) $\rho_{s}=910 \mathrm{kgm}^{-3}$ and (b) $\rho_{s}=6825 \mathrm{kgm}^{-3}$ at a strain rate $\dot{\varepsilon}=1.0 \times 10^{5}$ $s^{-1}$ with $t_{R}=9.3 \mu s$. Degree of deformation in each cell is represented by a gray scale varying from 1 (no deformation) to 0 (full collapse). The cell size of the specimen given as $d_{a v}=25.2 \mu \mathrm{m} . \ldots$. . . . .

4.17 Stress-strain response of a cellular material for different randomness at strain rate $\dot{\varepsilon}=1.0 \times 10^{5} \mathrm{~s}^{-1}$ and for pulse rise time $t_{R}=9.3 \mu \mathrm{s}$. The cell

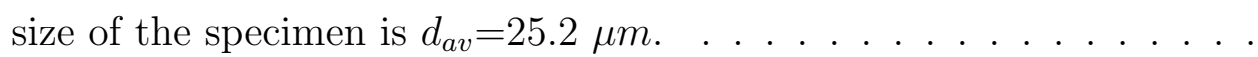

4.18 Snapshots of deformed specimens at selected time intervals for (a) $S_{d}=0$, (b) $S_{d}=0.85 \mu \mathrm{m}$ and (b) $S_{d}=3.0 \mu \mathrm{m}$ at a strain rate $\dot{\varepsilon}=1.0 \times 10^{5}$ $s^{-1}$ with $t_{R}=9.3 \mu s . \ldots \ldots \ldots \ldots \ldots$

4.19 Stress strain response of cellular specimens with different cell irregularity subjected to loading in different directions. . . . . . . . . . .

4.20 Snapshots of deformed specimens when impacted from the top for (a) perfect honeycomb $S_{d}=0.0$ and (b) highly random microstructure $S_{d}=0.3 \mu \mathrm{m}$ at a strain rate $\dot{\varepsilon}=1.0 \times 10^{5} \mathrm{~s}^{-1}$ with $t_{R}=9.3 \mu \mathrm{s} . \ldots$. . .

5.1 Schematic representation of byssus thread showing different regions (proximal thread, distal thread and adhesive plaque). The geometric and material properties of these regions are shown in the figure. The constitutive laws of distal and proximal threads have been illustrated in the top figure. Dashed line in the constitutive law represents the idealized response of the distal portion. . . . . . . . . . . . . .

5.2 (a) Force vs total thread length $(L+\Delta)$ of a mussel byssus thread of species $M$. californianus. Shaded region represents the extent of tri-phasic response predicted by the model. (b) Individual extension of the proximal $\left(\Delta_{p}\right)$ and distal $\left(\Delta_{d}\right)$ regions due to applied tensile displacement $\Delta . \ldots \ldots \ldots \ldots 112$ 
5.3 Steps of optimization formulation. Objective function of GA is evaluated through the finite element simulation. . . . . . . . . . .

5.4 (a) Variation of toughness $(U)$ of the whole thread for different percentage of proximal thread length $\xi$. (b) A map presents the condition at which maximum stress $\left(\sigma_{f}\right)$ and maximum extensibility $\left(\varepsilon_{\max }\right)$ criteria are achieved for different percentage of proximal thread length $\xi$. Types of overall deformation behavior such as tri-phasic $(A)$, bi-phasic $(B)$ and mono-phasic $(C)$ responses of the whole thread for different values of $\xi$ are also indicated. . . . . . . . . . . . . .

5.5 Variation of optimal percentage of proximal thread length $\xi^{*}$ for different ratios of Young's modulus of distal thread to proximal thread $(\chi)$ with a wide range of $\sigma_{y} / E_{p} \ldots \ldots \ldots$. . . . . . . . . .

5.6 Optimal surface of percentage of proximal thread length $\xi$ for different $\chi$ and ratios of failure stress to Young's modulus of proximal thread $\sigma_{f} / E_{p} \ldots \ldots \ldots \ldots \ldots \ldots$

5.7 Variation of optimal percentage of proximal thread length $\xi^{*}$ with $\sigma_{f} / E_{p}$ for $M$. galloprovincialis. Shaded region represents the extent of optimal percentage of proximal thread length as predicted by the model. Experimentally observed percentage of proximal thread length is also indicated on the plot. . . . . . . . . . . . .

5.8 Comparison of (a) percentage of proximal thread length and (b) maximum toughness as predicted by the model with experimentally observed values for different mussel species. Simulations are performed taking into account experimental variations of model parameters resulting in variation in the simulation results denoted by error bars. . . 
6.1 A representative design for a two phase material showing phase 1 material (epoxy matrix) in light gray and phase 2 material (A-glass particle) in dark gray. FEM mesh used for the simulations and the coordinate directions are also shown . . . . . . . . . . . . . . 140

6.2 Flow chart showing basic elements of surrogate model based feasibility analysis of the heterogeneous materials. . . . . . . . . . . .

6.3 Grid of input sample points over which HDMR component functions are evaluated by performing FEM simulations. . . . . . . . . . . . . 142

6.4 Comparison of FEM computed feasible region with the HDMR predic-

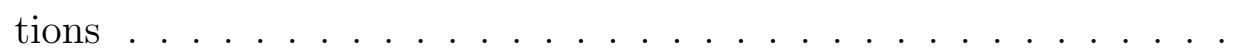

6.5 Comparison of HDMR predicted feasible region with FEM computed result (a) when $\varepsilon_{22}^{0}$ is fixed, and (b) when $\varepsilon_{11}^{0}$ is fixed. . . . . . . . . . 143

6.6 Schematics of Macro-and micro-scale boundary value problems and their coupling. . . . . . . . . . . . . . . . .

6.7 Representative volume element consisting softer matrix and harder particles of different radii as well as different stiffness. Particles are denoted by darker regions, darker shade denoting higher stiffness. . .

6.8 Stress contour of (a) ${ }^{1} P_{11}$, (b) ${ }^{1} P_{22}$, (c) ${ }^{1} P_{12}$, and (d) ${ }^{1} P_{21}$ for the RVE when subjected to macroscopic deformation gradient $\bar{F}_{11}=\bar{F}_{22}=1.3$, and $\bar{F}_{12}=\bar{F}_{21}=0.1 \ldots \ldots \ldots \ldots \ldots$

6.9 Simulated first order components of average microstress $\left({ }^{1} \bar{P}_{11}\right)$ with respect to the input variables. . . . . . . . . . . . .

6.10 Simulated second order components of average microstress $\left({ }^{1} \bar{P}_{11}\right)$ with respect to the components of macroscopic deformation gradient tensor. 156

6.11 (a) Evolution of proportionally loaded macroscopic deformation gradient. (b) Comparison of predicted first Piola-Kirchhoff (PK-I) stresses with full field finite element simulation. . . . . . . . . . . . . 157 
6.12 (a) Evolution of non-proportionally loaded macroscopic deformation gradient. (b) Comparison of predicted first Piola-Kirchhoff (PK-I) stresses with full field finite element simulation. . . . . . . . . . . . . 158

6.13 (a) Evolution of macroscopic deformation gradient with series of loading and unloading events. (b) Comparison of predicted first PiolaKirchhoff (PK-I) stresses with full field finite element simulation. . . . 159

6.14 Representative volume element consisting softer matrix and short fiber. Fiber is denoted by darker regions, darker shade denoting higher stiffness.160

6.15 Comparison of predicted first Piola-Kirchhoff (PK-I) stresses with full field finite element simulation for (a) proportional loading, (b) nonproportional loading, and (c) complex loading-unloading. . . . . . . . . . 161

6.16 Three dimensional representative volume element consisting softer matrix and a platelet. Platelet is denoted by darker regions, darker shade denoting higher stiffness. . . . . . . . . . . . . . . . . .

6.17 (a) Evolution of proportional type macroscopic deformation gradient.

(b) Comparison of predicted first Piola-Kirchhoff (PK-I) stresses with full field finite element simulation. . . . . . . . . . . . . .

6.18 (a) Evolution of macroscopic deformation gradient with series of loading and unloading events. (b) Comparison of predicted first PiolaKirchhoff (PK-I) stresses with full field finite element simulation. . . . 164

A.1 (a) Magnified view of undeformed and deformed cell of a cellular specimen made of polyethylene when subject to an impact load from top (Pal et al., 2010). (b) Left figure shows normalized lateral displacement history of end points of segment 'a'. Right figure depicts the normalized relative vertical displacement history of point $\mathrm{P}$ with respect to $\mathrm{Q}$ of the strut segment. . . . . . . . . . . . . . . . . . .

A.2 Schematic representation of deformation of strut when subjected to a vertical impact velocity $v \ldots \ldots \ldots \ldots \ldots \ldots$ 
A.3 History of normalized axial compression $(u / l)$, normalized transverse displacement $(w / l)$ and vertical force $\left(F_{v}\right)$ experienced by the strut. Phase I corresponds to the microinertial enhancement, whereas Phase II indicates the microbuckling of the strut. . . . . . . . . . . .

A.4 (a) History of normalized axial shortening $(u / l)$ (b) normalized transverse displacement $(w / l)$, and (c) vertical force-displacement responses for different impact velocities. The transition from phase I (microinertial enhancement) to phase II (microbuckling) is indicated by solid circle. The subregimes of phase I is also marked by solid squares. . .

A.5 (a) Quasi-static collapse force $F_{q s}$ for different half strut length $l$. (b) Microinertial enhancement of collapse force $\Delta F / F_{q s}$ for different impact velocities with different strut length. . . . . . . . . . . .

A.6 (a) Quasi-static collapse force $F_{q s}$ for different misalignment angles $\theta_{0}$. (b) Microinertial enhancement of collapse force $\Delta F / F_{q s}$ for different impact velocities $v$ with different misalignment angles $\theta_{0} \ldots$. . . . .

A.7 Vertical force-displacement response for different bulk material densities.198

A.8 (a) Quasi-static collapse force $F_{q s}$ for different bulk material density $\rho_{s}$. (b) Microinertial enhancement of collapse force $\Delta F / F_{q s}$ for different impact velocities $v$ with different bulk material density. . . . . . . . . 200

A.9 (a) Quasi-static collapse force $F_{q s}$ for different Young's modulus of the strut $E$. (b) Microinertial enhancement of collapse force $\Delta F / F_{q s}$ for different impact velocities with different Young's modulus. . . . . . .

A.10 (a) Effect of material viscosity $\eta$ on force-displacement response at higher impact velocity $v=1.0 \mathrm{~m} / \mathrm{s}$. (b) Microinertial enhancement of collapse force $\Delta F / F_{q s}$ for different viscosity $\eta$. . . . . . . . . . . . . 202

B.1 (a) Hexagonal lattice (unperturbed) on $x-y$ plane. (b) Constructed voronoi diagram on perturbed seeds. Window represents the actual cellular domain taken the diagram. . . . . . . . . . . . . 207 
C.1 Bilinear elastic-plastic constitutive law. . . . . . . . . . . . . . 209

C.2 Backward-Euler scheme. AB corresponds to elastic forward steps, and BC corresponds to the return map on the yield surface. . . . . . . . . 210

F.1 Zoomed undeformed and deformed shape of a cell. . . . . . . . . 226 


\section{List of Tables}

$3.1 R_{r m s}$ values for chosen combinations of $\bar{d}_{a v}$ and $\bar{\kappa} . \ldots \ldots 6$

$3.2 R_{r m s}$ values for different $\bar{v}$ and $\bar{\rho}$ as chosen from Figure 3.12 . . . . . 68

5.1 List of different GA parameters. . . . . . . . . . . . . 115

5.2 Material parameters for different mussel species. $\chi$ is evaluated considering the mean value of $E_{d}$ and $E_{p} \ldots \ldots \ldots$

6.1 Material properties .................... 153

6.2 Comparison of computational cost for each load step . . . . . . . . 165

E.1 Genetic algorithm terminology . . . . . . . . . . . . . 220 
xxvi 


\section{List of Algorithms}

1 Explicit dynamic corotational beam formulation. . . . . . . . . . 34

2 Implicit quasi-static corotational beam formulation. . . . . . . . . . . 36

3 Backward-Euler plasticity algorithm. . . . . . . . . . . . . 212 


\section{Acknowledgments}

I would like to express my deepest gratitude to my advisor Dr. Spandan Maiti for his guidance and support. I am thankful to him for this challenging research topic which helped me to learn the fundamentals of computational solid mechanics. I really appreciate the independent work environment which led my strong professional skills.

I would like to acknowledge my committee members Dr. G.M. Odegard, Dr. T.L. Haut Donahue and Dr. Yue Li for their time and valuable advice. I am thankful to Dr. T.L. Haut Donahue and Dr. G.M. Odegard for their fruitful suggestion in my proposal defense. I am grateful to Dr. Yue Li for serving as a committee member within very short period of time.

I am thankful to Dr. Ipsita Banerjee, University of Pittsburgh, Pittsburgh for her help in understanding the feasibility analysis and optimization formulations. I am also grateful to Dr. Ghatu Subhash, University of Florida, Gainesville for his insightful comments in deformation behavior of cellular materials.

I am thankful to Department of Mechanical Engineering-Engineering Mechanics for giving me the opportunity of doctoral study. I am grateful to West Engineering Computer Network (WECN) for providing computing facilities.

I would like to thank Dr. D.V. Kubair and Dr. D. Datta for their inspiration and encouragement to join doctoral studies program.

I would like to thank past and present members of our research group; Aniket, James, Siddharth, Sachin, Yinan, Swetha and Phillipe for their support and discussions and sharing wonderful moments during last four years. I would like to thank all the members of solid mechanics seminar group for conducting presentations, sharing the ideas 
and valuable comments and suggestions. I would like to thank all of my friends in Houghton; Parimal, Ananya, Patha, Dipankar, Subhasish, Swarup, Saikat, Anirudhha, Shreehari, Pavan, Jayeeta, Colina and Puspa for making my stay enjoyable and memorable.

I would like to acknowledge my family for their constant support, inspiration and encouragement till the date. I truly admire their patience during all of the past years. 


\section{Abstract}

Heterogeneous materials are ubiquitous in nature and as synthetic materials. These materials provide unique combination of desirable mechanical properties emerging from its heterogeneities at different length scales. Future structural and technological applications will require the development of advanced light weight materials with superior strength and toughness. Cost effective design of the advanced high performance synthetic materials by tailoring their microstructure is the challenge facing the materials design community. Prior knowledge of structure-property relationships for these materials is imperative for optimal design. Thus, understanding such relationships for heterogeneous materials is of primary interest. Furthermore, computational burden is becoming critical concern in several areas of heterogeneous materials design. Therefore, computationally efficient and accurate predictive tools are highly essential.

In the present study, we mainly focus on mechanical behavior of soft cellular materials and tough biological material such as mussel byssus thread. Cellular materials exhibit microstructural heterogeneity by interconnected network of same material phase. However, mussel byssus thread comprises of two distinct material phases. A robust numerical framework is developed to investigate the micromechanisms behind the macroscopic response of both of these materials. Using this framework, effect of microstuctural parameters has been addressed on the stress state of cellular specimens during split Hopkinson pressure bar test. A voronoi tessellation based algorithm has been developed to simulate the cellular microstructure. Micromechanisms (microinertia, microbuckling and microbending) governing macroscopic behavior of cellular solids are investigated thoroughly with respect to various microstructural and loading parameters. To understand the origin of high toughness of mussel byssus thread, a Genetic Algorithm (GA) based optimization framework has been developed. It is found that two different material phases (collagens) of mussel byssus thread are opti- 
mally distributed along the thread. These applications demonstrate that the presence of heterogeneity in the system demands high computational resources for simulation and modeling. Thus, Higher Dimensional Model Representation (HDMR) based surrogate modeling concept has been proposed to reduce computational complexity. The applicability of such methodology has been demonstrated in failure envelope construction and in multiscale finite element techniques. It is observed that surrogate based model can capture the behavior of complex material systems with sufficient accuracy. The computational algorithms presented in this thesis will further pave the way for accurate prediction of macroscopic deformation behavior of various class of advanced materials from their measurable microstructural features at a reasonable computational cost. 


\section{Chapter 1}

\section{Introduction}

With the advent of composite materials, heterogeneous materials are finding increasing applications in different fields of engineering and technology. Though homogeneous bulk materials such as metals and ceramics are still a major component for different mechanical applications, heterogeneous materials systems incorporating multiple laminates, second phase particles, grain boundaries and architectural details are gaining importance due to their superior strength, thermal stability, tailorability and multifunctionality. For example, commercial airplanes were made of metals up to 70 's, but now they employ composite material in their structural components to reduce the weight significantly ( 11\% weight reduction in Boeing 777 (Smith, 2003)).

Designing a heterogeneous material is however still a challenge. Myriad design philosophies can be applied to achieve a set of requisite functionalities and properties. The latest trend is to mimic the design of biological materials, that are highly heterogeneous and optimized for a specific set of performance. In this thesis we will focus on the origin of strength and toughness characteristics of cellular solids and tough biological materials. We will study and quantify the measurable features of these 
materials systems at the microscopic level, and will predict the resulting deformation and failure behavior at the macroscopic level.

\subsection{Heterogeneous materials and their technolog- ical importance}

Heterogeneous materials can be composed of different materials or phases such as composites, same material with large amount of porosity (e.g., cellular solids) or the same material in different states such as polycrystal. Such materials abound in the form of synthetic materials and in nature. Examples of synthetic heterogeneous materials include composites, cellular solids, gels and concretes. Natural heterogeneous materials comprise of polycrystals, soil, granular media, wood, bone, blood, animal and plant tissue and biological cells (Torquato, 2001; Buryanchenko, 2007), to name a few. The heterogeneous materials are known to exhibit superior properties in applications where multiple functional requirements are simultaneously expected. Different properties and advantages of various components can be fully exploited by introducing material heterogeneity. A variety of applications involving heterogeneous materials can be found in mechanical, electrical, thermal, optical, biomedical, geophysical and other fields in past 40 years. These materials are widely being used as structural components in aerospace, mechanical and civil engineering where the applications are driven by defense, space, consumer industries, aircraft, space vehicles, automobiles, building construction industries, infrastructures etc. However, these materials are highly complex due to their microstructural variations. Thus, determination of mechanical properties of these complex materials has attracted considerable research efforts in the recent past. Among different class of heterogeneous materials, cellular solids, structural biological materials and multiphase composites are finding 
increasing technological importance owing to their unique combination of mechanical properties: mainly strength, stiffness and toughness characteristics. In the following we briefly discuss these materials.

\subsubsection{Cellular materials}

Cellular materials are regarded as porous materials with very large porosity or very low solid volume fraction. Interconnected network of solid struts or faces forms the cellular architecture. These materials are ubiquitous in nature and man-made materials (Gibson and Ashby, 1997; Hilyard, 1982). Biological as well as inorganic materials can exhibit cellular architecture at different length scales, ranging from cytoskeleton networks in eukaryotic cells to cellular organization of different biomaterials (such as wood, bone and sponge), to synthetic foams for different structural applications. Cellular microstructure renders multifuctionality to the structure/system while providing a lightweight design. Cellular microstructure typically provides superior specific strength and toughness. Synthetic cellular solids (foams) are generally made by dispersing gas into liquid medium and then cooling it to solidify. Typically, cellular solids are classified as open cell and closed cell cellular material. Based on the bulk materials that are transformed to cellular solids, they are categorized as polymeric, metallic and ceramic foams. Owing to their light weight, good shock absorption capability and ease of fabrication, cellular materials are widely used as advanced structural components in aircraft and automobile industries and as shock absorbing packaging elements for electronic components. However, cellular materials are highly heterogeneous, and can exhibit a wide set of statistical variabilities. Predicting macroscopic mechanical behavior of these materials is an enormous challenge facing material designers and product developers. Development of highly energy efficient cellular structure requires in-depth understanding of energy absorption characteristics arising from its inherent 
microstructural variations especially in a high impact loading scenario.

\subsubsection{Structural biomimetic materials}

Future generation engineering materials must exhibit physical (mechanical, electrical and thermal) and chemical (adhesive, reactive) properties optimized for particular applications. In this context, biomimicry, a new paradigm in material design, provides a powerful tool for the development of advanced materials. However, biological materials are multifuctional: Design principles behind a particular functionality is extremely difficult to identify. From an engineering materials point of view, we are mostly interested in biological materials offering high strength, stiffness and toughness owing to their smart design. Biological mineralized composites such as bone, dentin, sea shells and sponge spicule offer higher values of fracture toughness although their constituent materials are brittle. A prime example is nacre, which consists of 95 vol.\% of layered aragonite $\left(\mathrm{CaCO}_{3}\right)$ platelets attached by a thin layer of organic materials, while ex-

hibiting a toughness several order higher than that of $\mathrm{CaCO}_{3}$ (Espinosa et al., 2009). Biological polymer composites such as ligaments, silk and collagen offer remarkable strength, toughness and extensibility. For example, spider dragline silk is considered as nature's high performance fiber with exceptional combination of the above properties such as Young's modulus of 10-50 GPa, elongation to break of 10-30\% and tensile strength of 1.1-1.4 GPa (van Hest and Tirrel, 2001). Mussel byssus thread, another interesting biomaterial, also provides high specific strength, toughness and extensibility to ensure its attachment with underwater surfaces (Gosline et al., 2002). Such biological filaments can be a potential candidate for biomimetic development of tough fiber and fabrics. The mussel byssus thread is composed of three distinct components, namely the proximal region, distal region and adhesive plaque. The proximal region is short and softer, while the distal region is long and much stiffer. 
The whole thread when subjected to tensile load shows a triphasic response rendering high overall toughness and extensibility. However, the distribution of the thread components behind the origin of such properties has never been addressed.

\subsection{Computational mechanics applied to the de- formation and failure response of heterogeneous materials}

Modern technology necessitates the development of advanced high performance light weight materials with remarkable strength, stiffness and toughness. However, achieving all of these properties simultaneously possesses critical challenges. As outlined before, owing to strength, toughness and light weight, the cellular and composite materials are drawing their considerable attention within the material science and engineering community. However, fundamental challenges lie in manufacturing of these complex materials with the state of art in material science. The central paradigm of material science is to design the material with desired performance with a goal of reduced process cycle (cost effective design), as processing controls microstructure and ultimately the bulk properties of the heterogeneous materials. Microstructureproperty relationship for these complex materials needs to be thoroughly understood to fulfill this purpose. Present trends aim towards computational material design modeling and simulation (e.g. finite element methods, homogenization techniques, optimization method etc.) instead of costly iterative experimental procedures. Therefore, development of synthetic materials, advanced biomimetic materials and composite materials demands in-depth understanding of the macroscopic mechanical response stemming from the complex microstructure through an integrated and efficient computational framework. 


\subsubsection{Literature review}

\subsubsection{Dynamic response of cellular materials}

Several dynamic testing procedures such as drop-weight and air gun ballistic experiments have been utilized to determine dynamic response of different materials (Viot et al., 2008; Juntikka and Hallstron, 2004; Avalle et al., 2001). However, these techniques mainly provide energy absorbing characteristics of the material but not a complete stress-strain response. Split Hopkinson pressure bar (SHPB) technique, originally developed by Kolsky (1949), has been widely used to determine dynamic properties of various engineering materials. This particular technique has an advantage over above mentioned methods in determining the point wise dynamic constitutive response of materials averaged over its volume. Recently, SHPB test setup has been suitably modified to obtain accurate dynamic properties of different class of materials including metals (Gray, 2000), shape memory alloys (Chen et al., 2001), concrete (Ross et al., 1996), ceramics (Subhash and Ravichandran, 2000a), composites (Song et al., 2003), rubbers (Song and Chen, 2003; Shim and Mohr, 2008) and biological materials (Shergold et al., 2006).

SHPB technique is based on one dimensional wave propagation theory with the assumption that a uniformly deforming specimen is subjected to a dynamically equilibrated uniaxial stress state. This assumption ensures that the dynamic strain and stress of the specimen can be computed from measuring only reflected and transmitted signals. The dynamic equilibrium stress state is assumed to be achieved when the difference of forces at both ends of specimen is insignificant. However, for a soft cellular specimen, wave velocity is typically very low, and its amplitude attenuates significantly when traveling through a thick specimen thus restraining it from achieving dynamic equilibration quickly (Song et al., 2003). Moreover, the specimen 
can exhibit significant localized deformations (Chen et al., 2002a) during the early stage of wave propagation event further limiting it from achieving a uniform state of stress. Furthermore, impedance mismatch between the bars (incident and transmission) and the specimen is a key parameter to be determined for the effective dynamic testing of soft cellular specimens. A perfect impedance match between the specimen and bars will allow the entire incident wave to propagate into the transmission bar without any reflection thus requiring longer time to equilibrate the stress in the specimen. On the other hand, a high impedance mismatch expedites the equilibrium process (Yang and Shim, 2005) due to reflection of wave with larger amplitude but the transmitted signal becomes too weak leading severe problem in measurement of stress in transmission bar (Chen et al., 1999). As a consequence of these difficulties in establishing a one dimensional stress state in the specimen, standard data reduction routines for split Hopkinson pressure bar that rely on stress equilibration cannot be used directly for the case of polymeric foams. Mousavi et al. (2005) developed SHPB procedures for viscoelastic material which do not require stress equilibrium with in specimen. These procedures are valid only when the specimen is short and specimen to bar impedance is low.

To deal with such challenges, numerous modifications have been proposed to the traditional SHPB technique (Subhash et al., 2006a; Liu and Subhash, 2006; Chen et al., 2002c; Tagarielli et al., 2008) to obtain the dynamic response of different low impedance cellular solids such as polymeric foams. Chen et al. (1999) used hollow aluminum bars to reduce the impedance mismatch at the interfaces, whereas, Subhash et al. (2006a) used polymeric bars along with a novel data reduction procedure to derive the dynamic response of polymeric foams. To alleviate the problem of achieving stress equilibration in a thick specimen, Song and Chen (2004) suggested a reduction in specimen length for low impedance materials. Along with thickness reduction, they also suggested a pulse shaping technique (Chen et al., 2002a) to achieve equilibra- 
tion for a soft material. A pulse shaper in the SHPB set up essentially increases the rise time of the loading pulse and thus facilitates to achieve homogeneous deformation of the specimen as well as dynamic stress equilibration. The above techniques have been also employed in several other class of materials (Vecchio and Jiang, 2007; Frew et al., 2006, 2005). The above modifications in the SHPB setup have also been incorporated in dynamic testing of several soft materials (Nie et al., 2009) as well as extra-soft specimen such as ballistic gelatin and biological tissues (Song et al., 2007). Furthermore, Song et al. (2008) also developed a long split Hopkinson pressure bar to characterize the soft materials at intermediate strain rates.

Energy absorption characteristics of cellular materials and their relationship to the microscopic deformation behavior of individual cells have been extensively studied in the past decade. Wu and Jiang (1997) experimentally studied the effect of cell dimension, material strength and sandwich core thickness on the crushing phenomenon of aluminum foams. They suggested that a honeycomb structure with a smaller cell size and stronger material offers better energy absorption capability. Daxner et al. (1999) investigated the effect of mesoscale inhomogeneities of cellular material density on the energy absorption behavior of aluminum foam. It was found that strong meso-inhomogeneities lead to strain localization which reduces the initial plateau stress. Yi et al. (2001) experimentally showed that yield strength as well as energy absorption of open cell aluminum foams increase with strain rate. Using the split Hopkinson pressure bar (SHPB), Chakravarty et al. (2003) found that peak stress and absorbed energy of poly-vinyl chloride (PVC) foams are dependent on foam density and strain rate. They also developed a model based on a unit cell geometry of the closed-cell foams to predict the energy absorption at high strain rates. Subhash et al. (2006b) also found similar increase in collapse stress of epoxy foam at higher strain rate. Vural and Ravichandran (2003) experimentally investigated the compressive strength, plateau stress, densification strain and energy dissipation capacity of balsa wood with different densities at high strain rates. They found that initial failure 
strength of balsa wood is very sensitive to the rate of loading while plateau stress remains unaffected by the strain rate. Subhash and Liu (2004) studied crushability characteristics of epoxy foams and developed 'crushability maps' for cellular materials. A crushability map depicts the evolution of porosity, density and energy absorption capability of foams as functions of strain, stress and porosity. Saha et al. (2005) experimentally found that energy absorption of closed cell poly-vinyl chloride (PVC) and polyurethane foam depends on their density, microstructure and applied strain rate. Hönig and Stronge $(2002 \mathrm{a}, \mathrm{b})$ studied dynamics of crush band initiation and wave trapping behavior of honeycombs under in-plane impact loading. Their simulations revealed the existence of a critical impact velocity beyond which a crushing band is initiated at the impact end and a corresponding increase in total dissipation energy. They mentioned that this fact could be attributed to the cells collapsing in a symmetric crushing mode. To understand the effect of cell irregularity along with impact velocity on deformation behavior of cellular materials, Zheng et al. (2005b) developed a deformation mechanism map. They also mentioned that relative energy absorption capacity of cellular materials can be improved by increasing their cell irregularity.

A number of mechanisms such as strain rate sensitivity of the constitutive material, effect of microinertia, microbuckling and associated bending of cell walls, and shock enhancement at high strain rate has been suggested in the literature to explain the rate effect in cellular materials as observed in dynamic experiments. Lateral inertia of a strut, known as the microinertia effect, can significantly enhance its dynamic buckling stress (Calladine and English, 1984) thus showing an overall increase in the stress response of the cellular material. On the contrary, Deshpande and Fleck (2000) suggested that microinertia effects may be negligible for foams as they exhibit a well defined plateau in their stress-strain response typical of a Type I structure. However, a single cell, when subjected to a dynamic loading, exhibits a strong softening behavior immediately after achieving the peak stress. This behavior is reminiscent 
of a Type II structure that is known to exhibit strong strain rate sensitivity. Thus, the local behavior of the cellular material at the cell level may exhibit considerable rate effect due to microinertia that may not manifest itself at the specimen level due to the complex interplay between cell wall bending, microbuckling and microinertia phenomena. The observed rate sensitivity can also be attributed to the presence of a shock front in the deforming domain at high velocity impact (Tan et al., 2002; Harrigan et al., 1999; Elnasri et al., 2007; Pattofatto et al., 2007). These researchers have also suggested that early peak load occurs in the constitutive response due to the lateral inertia of the cell walls that in turn depends on the material properties of the wall. Nemat-Nasser et al. (2007) have observed the dependence of the location of localized deformation on the impact velocity during high-rate inertial loading.

\subsubsection{Mechanical behavior of mussel byssus thread}

Byssus threads are produced by mussels to provide secure anchorage with rocks, reefs or other shelled organisms in a wave swept environment. These threads are one of the exquisitely engineered materials by nature that provide specific mechanical requirements of stiffness, strength, toughness and extensibility (Alper, 2002) in order to ensure their survival in a harsh environment. Toughness value of an intact byssus thread $\left(12.5 \mathrm{MJ} / \mathrm{m}^{3}\right)$ is higher in comparison to tendon $\left(2-5 \mathrm{MJ} / \mathrm{m}^{3}\right)$ is lower than silk (50-180MJ/m3) (Denny, 1988) and is comparable to kevlar (Gosline et al., 2002), a widely used polymer in protective engineering components. Typically, 2060 threads (Bell and Gosline, 1997) are engaged in tethering the mussel to a hard surface through their adhesive plaques. The adhesive plaque produces strong and flexible glue rapidly and allows the mussel to adhere with any surface under ambient conditions of sea water (Waite et al., 2005; Silverman and Roberto, 2007). Unique combination of strength and toughness of these threads as well as underwater adhesive properties of plaque has recently generated considerable attention in the field of 
biomimetic applications (Hearle, 2007; van Hest and Tirrel, 2001; Scheibel, 2005).

A typical byssus thread comprises of three regions: (1) proximal region consisting of corrugated surface, (2) distal region which is smoother, and (3) adhesive plaque which is a small region attached to the hard surface under water. The macroscopic structure of proximal thread is similar to a fiber reinforced composite with collagen containing fibrils and soft proteinious matrix, while the distal portion comprises of densely packed fibrous bundles. The distal region of the thread is highly stiff and less extensible, whereas the proximal region shows an elastomeric response undergoing a large extension prior to failure.

The origin of the high attachment strength of mussel byssus thread can be attributed to plaque adhesive proteins. To date, five protein families designated as Mytilus edulis foot proteins (Mefp- $N$, where $N$ indicates the chronological order of discovery) are known to be present within the adhesive plaque (Wiegemann, 2005). Mefp-1 forms a protective coating (Rzepecki et al., 1992) to the threads as well as plaque while Mefp-2 and Mefp-4 (Rzepecki et al., 1992; Vreeland et al., 1998) are attributed to a solid foam like interior of the plaque. The remaining protein such as Mefp-3 and Mefp-5 (Papov et al., 1995; Warner and Waite, 1999; Waite and Qin, 2001) function as a primer that adheres the plaque to the surface. However, Lin et al. (2007) found that a typical shape of plaques contributes to a high adhesive strength rather than due to the higher surface energy of mussel foot proteins. Consequently, the other source of the strength is thought to be from the thread itself. To explain the origin of high attachment strength of the mussel byssus thread, numerous studies examined the morphology of the thread (Coyne et al., 1997; Qin et al., 1997; Waite et al., 2002; Harrington and Waite, 2007, 2009). They found that collageneous protein; preCol$\mathrm{P}$ and preCol-D are mainly present in proximal and distal portions of the thread and are distributed in a graded fashion, whereas preCol-NG is uniformly distributed along the thread. Each preCol is a natural block copolymer sequence with three ma- 
jor domains: a central collagen domain consisting of triple helix motif Gly-Xaa-Yaa (Xaa preferably Pro or Gly residues), flanking elastic domain (soft segment), and histidine-rich terminal domain. The lateral and longitudinal assembly of triple helices either through metal-chelation or chemical bonding by virtue of disulfide bridges and diDOPA formation (Hagenau et al., 2008; Zhao et al., 2006) results in the typical macroscopic responses of proximal and distal regions as found experimentally. On the other hand, slippage or dislocation within this cross-linking or crystalline sites (Vaccaro and Waite, 2001; Harrington and Waite, 2007) results in the yielding and self-healing of distal thread. Vaccaro and Waite (2001) found that application of urea, dithiothreitol (DTT), and metal chelating agents greatly affects the modulus, yield point and the energy dissipation. Hence, understanding the manifestation of chemical composition to the mechanical properties is of utmost importance in studying byssus threads.

There have been several parallel efforts in both experimental determination of tensile properties (Bell and Gosline, 1996; Lucas et al., 2002; Braze and Carrington, 2006) of the thread with mechanistic modeling (Bertoldi and Boyce, 2007; Troncoso et al., 2008) of its deformation behavior. Experimental observations show that during tensile tests, most of byssus threads from different mussel species offer a characteristic triphasic response: an initial stiff phase, followed by a more extensible yield phase, which is further followed by a second stiff phase. According to Bell and Gosline (1996), the yielding and extensibility of mussel thread enhance its ultimate attachment strength allowing other threads to share the tensile load and thus, it secures dislodgement from the rock surface. The yielding behavior of byssus thread critically depends on its interaction with water (Aldred et al., 2007; Troncoso et al., 2008). Carrington and Gosline (2004) experimentally observed an increase in the stiffness as well as the yield stress of the thread when it was stretched under higher loading rates. The constitutive model as developed by Bertoldi and Boyce (2007) addressed the similar observation where the origin of its loading rate dependency is attributed from the entropic con- 
tribution in the ultrastructure of distal fibers. Moeser and Carrington (2006) showed that wave action is not only the contributing factors for the attachment strength. In another study, Moeser and Carrington (2006) observed that the tensile mechanical properties of the individual byssus thread and deterioration of thread quality greatly affect the attachment strength. However, the origin of strength and toughness and its dependence on the heterogeneous microstructure have not been investigated yet.

\subsubsection{Computational complexity for the simulation of heterogeneous materials response}

Understanding the contribution of heterogeneities at different scales of a heterogeneous medium is key to design advanced materials such as composites in high performance applications. The goal of material science is to develop a comprehensive understanding of microstructure-properties-function relationship in order to systematically design the material with specific desired properties. Although a multitude of homogenization techniques are available for material design, those are limited to heterogeneous materials with linear constituents, and statistically homogeneous materials (Kanoute et al., 2009). These techniques are often lacking in a complex loading scenario as well as in finite deformation regimes. A comprehensive foundation of the homogenization of heterogeneous materials are illustrated by Wills (1981), Muller (1987), Nemat-Nasser and Hori (1993), Castaneda and Suquet (1998) and Miche et al. (1999). Although these methods offer estimation or the bound of effective properties or exact results, they are not sufficient to analyze statistically heterogeneous microstructure subjected to arbitrary macroscopic loading. In case of hyperelastic media, Hasin (1985) provided some estimates for special type of loading. Ogden (1978) and Castaneda (1989) determined the bounds on the overall strain energy function of geometrically nonlinear structures. In case of finite deformation, Castaneda and Tiberio (2000) found variational estimates for the effective or 
homogenized stored energy function of hyperelastic composites through the generalization of second-order procedure as developed by Castaneda (1996). Furthermore, Lopez-Pamies and Castaneda (2004) found the bounds of hyperelastic porous material with random microstructure subjected to finite strain.

Computational or incremental homogenization techniques developed by several authors (Guedes and Kikuchi (1990), Ghosh et al. (2001), Smit et al. (1998), Feyel (2003), Michel et al. (1999), Feyel and Chaboche (2000), Terada and Kikuchi (2001), Andrade and Tu (2009), Kouznetsova et al. (2004), Miche (2003)) provide suitable approaches to analyze the macroscale model with complex microstructure. These methods depend on solving two nested boundary value problems (BVP), one in macroscale and the other in microscale concurrently. Macroscopic deformation gradient/strain tensor at macroscopic material point is used as the kinematic boundary condition of the associated representative volume element (RVE). The microstructural BVP is solved and the stress-strain relationship at every macroscopic point is determined by averaging the microscopic stress tensor. The above methods provide the following benefits: (1) detailed microstructural information, including the evolution of microstructure can be incorporated in macroscopic analysis, (2) large deformation and rotation can be analyzed both at micro and macrolevel, (3) arbitrary behavior, including physically nonlinear and time dependent behavior can be incorporated to model the microlevel. Most of the above techniques assumed that the microstructural length scale is sifficiently small compared to the characteristic macro-structural length scale. Hence, these methods are called as first-order computational homogenization techniques. Kouznetsova et al. (2004) developed a second-order approach to simulate the boundary value problem where both length scales are comparable.

Although the above techniques are robust enough to analyze complex microstructure, the associated computational cost is tremendous. To indicate the computational complexity, consider a macro-scale domain with $n_{e l}$ finite elements, $n_{g}$ Gauss points per 
element, $n_{l}$ load increments in the macro-scale, and $N_{I C}$ and $N_{I m}$ average iterations in the macro- and micro scale, respectively. Consequently, the total number of linear solver calls for a micro-problem is $n_{e l} n_{g} n_{l} N_{I C} N_{I m}$, a large number of computation when the degrees-of-freedom of the RVE is substantial. Moreover, the data necessary for incremental resolutions to be stored for each problem demand a large amount hard drive space.

Two approaches have been proposed to overcome the above computational difficulties. First, the microlevel RVE simulations can be distributed on massively parallel computer nodes (Feyel and Chaboche, 2000). As the RVE simulations at given iteration can be performed independently, significant cost reduction is possible through this approach. Another approach is the reduction of microscopic model order by replacing the original RVE with a lower order approximation. A significant progress in lower order approximation has been made through voronoi cell method (Ghosh and Moorthy, 1995), Fourier transform (Moulinec and Suquet, 1998, 1994), spectral method (Aboudi, 1982), transformation field analysis based methods (Dvorak, 1992; Bahei-El-Din et al., 2004), network approximation method (Beryland and Kolpakov, 2001) and mathematical homogenization with eigenstrain (Fish et al., 1997, 1999; Fish and Shek, 1999). Recently developed eigendeformation based reduction methodologies proposed by Oskay and Fish (2007) and Oskay (2009) provide effective means to analyze the nonlinear heterogeneous systems through the model reduction. Also, McVeigh et al. (2006) developed an alternative approach based on multiresolution continuum theory to take into account nonhomogeneous deformation at different scales.

A novel approach proposed by several authors to solve a large nonlinear model is based on the proper orthogonal decomposition (POD) technique. This method is showing great promise in computing very large models resonably accurately without a great demand on computing resources. POD is a powerful and elegant method for data analysis, aimed at obtaining low dimensional approximate description of a 
higher dimensional process. Using this idea, Yvonnet and He (2007) proposed a reduced model multiscale method (R3M) for homogenization of nonlinear hyperelastic heterogeneous material at finite strain. Ryckelynck (2009) offered an improved saving of computational cost through the hyper-reduction of elastic-plastic model of a simple structure. Similarly, Niroomandi et al. (2010) developed a model reduction technique for hyperelastic materials with large strain where tangent stiffness matrices do not need to be updated. Proper orthogonal decomposition based reduced order modeling frameworks are widely used to analyze dynamics of nonlinear solids (Krysl et al., 2001; Lall et al., 2003) as well as robust design optimization (Weickum et al., 2009), where a repetitive evaluation of full model is required at each time step. Although the above methods are considered as moderately efficient approach, a significant computational cost reduction is still required to analyze the macroscopically heterogeneous media efficiently. Towards this end, Yvonnet et al. (2009) proposed a completely different approach where a look-up table of effective RVE response is generated over a grid of possible macroscopic loading space. The effective response of nonlinear heterogeneous material evaluated any point is constructed by interpolating the nodal effective responses via appropriate interpolation functions and their derivatives. Similarly, response surface methodology based on constructing global approximation of system behavior evaluated at various points in the design space is often used for structural optimization (Roux et al., 1998).

\subsubsection{Technological challenges}

\subsubsection{Computational frameworks for cellular and biological materials}

As discussed earlier, extraction of an accurate dynamic behavior in SHPB depends on the state of stress in the specimen. Thus, the condition at which either stress 
equilibrium or non-equilibrium prevails needs to be understood thoroughly. Prior knowledge of such a stress state will assist to determine whether a standard data reduction routine for a SHPB test or special methodologies, e.g., evaluation of SHPB response under the condition of non-equilibrium (Mousavi et al., 2005) stress state is applicable. The stress state may be highly dependent on the microstructural, material, and loading parameters. However, to date, no analysis is available to correlate microstructural parameters of the cellular solid to be tested (intrinsic to the material) such as cell size, cell wall thickness, and number of cells in the specimen length direction to the stress state of the specimen. In addition, other experimental parameters extrinsic to the test specimen such as initial loading rate, pulse shape, and boundary impedance mismatch between the specimen and the bars are known to influence SHPB test results significantly. Understanding the stress state with respect to these parameters either requires extensive experimental enumeration, or is impossible with the current state of art. Therefore, the present study aims to evaluate the effect of these parameters on the stress state of a cellular specimen in a SHPB setup from a computational viewpoint.

Furthermore, it can be found that strain rate sensitivity and the resulting effect on the energy absorption capability of cellular materials subjected to a dynamic loading depends on a number of intertwined mechanisms. Experimental evaluation of the effect of these mechanisms is extremely difficult due to variabilities in processing conditions during manufacture, variation in material and microstructural properties and the strong specimen size effect exhibited by these cellular materials. Moreover, observation of the effect of these phenomena separately is extremely difficult due to their strong interdependence. Simplified theoretical analyses may shed light on some of these mechanisms in an idealized setting. However, complexity of the microstructure and deformation behavior of these materials calls for sophisticated computational models. Our goal is to study the effect of different micromechanisms and microscopic geometric features on observed deformation behavior and constitutive response from 
a computational perspective.

As discussed earlier, mussel byssus thread provides superior combination of strength, toughness and extensibility owing to its structural heterogeneity, i.e., the combination of stiff distal thread and soft proximal thread. However, what percentage of distal or proximal thread length contributes such superior properties has never been investigated. Biomimetic development of tough thread requires a thorough understanding of such distribution of distal and proximal regions along the thread. Therefore, toughness characteristics of mussel byssus thread needs to be evaluated for a wide range of heterogeneity distribution. Moreover, the optimal distribution to achieve specific properties must be identified. Hence, a computational framework comprising of optimization methodology and mechanistic representation of mussel byssus thread is essential.

\subsubsection{Efficient predictive tools for complex materials}

Computational material models are typically extremely complex involving high computational cost. Advanced material design involves optimization of microstructures to the specific performance requirements, sensitivity analysis of constituents on its macroscopic behavior, feasibility analysis of the specific design under certain constraints, and multiscale approaches. All of these situations require multiple model simulations with a reasonable computational cost. However, associated computational complexity is still intractable. For all these situations, a surrogate model that will map the input and output space of the actual model accurately but at a fraction of original computational cost is highly desired. As found in literature, a class of methods involving model dimension reduction contributed towards this end. However, these methods invariably result in loss of information due to their representation in a reduced dimension. Therefore, a significant improvement in existing methodology 
is required to reduce the computational efforts arise in predicting the behavior of heterogeneous material design. The High Dimensional Model Representation (HDMR) technique, introduced by (Rabitz et al., 1998; Rabitz and Alis, 1999) is a promising alternate strategy designed to alleviate complex models of their computational burden by systemic sampling procedures to map out relationships between sets of input and output model variables. The HDMR technique is principally based on an alternate method of model representation, which preserves the dimensionality of the original problem, yet achieves drastic reduction in computational burden. However, the application of HDMR methodology in construction of surrogate mechanical model is quite new in the area of computational mechanics. The potential of such methodology needs to be examined in analyzing complex material systems towards an alternative avenue to develop highly accurate yet computationally tractable models.

\subsection{Research objectives}

In the present study, we envisage heterogeneity is the key at different length scales to achieve superior mechanical properties of complex materials. Correspondingly, there are three primary goals of the present study. First, we aim to understand the structure-property relationship of cellular materials through a computational framework. The model will be robust enough to accurately capture deformation kinetics and constitutive response of a representative cellular microstructure under impact loading. Mechanisms responsible for the origin of macroscopic behavior will be understood from its microstructural parameters. Next, we focus on the underlying design principle (structure-property relationship) governing the origin of the high toughness of mussel byssus thread. The primary interest is to identify the key material and geometric parameters through a computational approach. The proposed methodology comprises an optimization technique and a mechanistic representation of the thread. 
Last, we attempt to develop a novel methodology to efficiently predict the structureproperty relationship of highly complex heterogeneous materials in a computationally cheap manner. The main concern is to introduce surrogate model based approach that demands less amount of response evaluation but accurately predicts the behavior of a complex system. Towards these goals, several research tasks have been identified, as described below.

\section{Task I: Deformation mechanisms of soft cellular material for energy ab- sorption:}

- Development of finite element framework to simulate transient response of cellular microstructures.

- Computer generation of cellular microstructures and quantification of their randomness.

- Extension of numerical framework to simulate the testing of cellular specimen under split Hopkinson pressure bar (SHPB) setup.

- Identification of key micromechanism and their contribution towards energy absorption property.

- Investigation of mechanical behavior of cellular structural element for their microstructural parameters.

\section{Task II: Deformation mechanisms of tough biological thread:}

- Development and validation of computational model to characterize quasi-static deformation behavior of tough biological threads. 
- Incorporation of novel optimization technique along with mechanistic framework

- Determination of key thread parameters contributing towards toughness.

- Comparison of model predicted key parameters of the thread with its experimental counterpart.

Task III: Surrogate model based predictive tool for effective quantification of structure-property relationship in heterogeneous materials:

- Development of surrogate model based framework for efficient prediction of mechanical behavior of a complex material system.

- Development of computationally efficient feasibility analysis of heterogeneous materials.

- Development of efficient multiscale framework for heterogeneous material with hyperelastic constituents subjected to large deformation.

\subsection{Scope of the present study \& thesis organiza- tion}

The goal of the present study is to understand the micromechanics of two types of heterogeneous materials, namely cellular materials and mussel byssus thread, though a computational approach. Therefore, in Chapter 2, we present a numerical framework to study the dynamic response of the cellular materials and quasi-static response of heterogeneous filaments. A corotational beam formulation is adopted to accurately 
capture the large deformation kinetics. An explicit finite element scheme is developed to analyze the transient response of the cellular materials. The explicit dynamic corotational formulation has been validated against a known analytical solution. Next, we illustrated the detail steps to generate cellular microstructure based on voronoi tessellation. The randomness of cellular microstructure has been quantified. In Chapter 3, we mention the challenges involved in extracting the experimental response of a soft cellular material during impact loading. Using the corotational beam based explicit dynamic formulation, we delineate the effect of intrinsic and extrinsic parameters on the stress state of a cellular specimen during SHPB test. Furthermore, the corotational beam based formulation has been used in Chapter 4 to study the contribution of different micromechanisms on the overall behavior of cellular material under dynamic impact. The effect of cellular microstructural parameters on these micromechanisms is thoroughly investigated. In Chapter 5, a methodology is proposed to investigate the principle behind the origin of high toughness of mussel byssus thread. This methodology is based on an optimization framework along with mechanistic representation of mussel byssus thread. An implicit corotational beam framework has been used to find the response of the byssus thread. The Genetic Algorithm (GA) based optimization framework provides us the key parameters responsible for maximum toughness. In Chapter 6, we proposed Higher Dimensional Model Representation (HDMR) based surrogate model to reduce computational burden arising in analysis of heterogeneous materials. The applicability of such model has been demonstrated for construction of failure envelop of a heterogeneous material performing a few finite element simulations. Furthermore, the HDMR based surrogate model has been applied in two scale finite element framework for heterogeneous material with nonlinear hyperelastic constituents undergoing large deformation. Finally, in Chapter 7, we concluded our findings and demonstrated the future directions of the present research work. 


\section{Chapter 2}

\section{Development of computational framework}

Main objective of the present analysis is to study the deformation characteristics of open cell cellular solids and biological filaments. Both can be computationally represented by a nonlinear beam at the microlevel. For open cell cellular solids, we are specifically interested on the effect of microstructural parameters on its dynamic response. Furthermore, we intend to investigate the contribution of material heterogeneity on strength, toughness and extensibility criteria of tough biological filament. Towards that end, a computational scheme that accounts for microstructural, inertial and finite kinematic effects during dynamic deformation of cellular solids has been developed. Next, this computational scheme is further modified to study the mechanical behavior of mussel byssus filament subjected to quasi-static loading. Therefore, the key ingredients of the proposed scheme are (1) highly accurate finite deformation beam formulation that captures correct deformation of cellular structural components, (2) finite element framework to simulate dynamic deformation characteristics of cellular solids, (3) finite element framework to simulate quasi-static response of 
biological filament, and (4) computer generation of open cell cellular microstructure.

\subsection{Corotational beam formulation}

Open cell soft cellular solids can exhibit complex deformation behavior as the struts undergo large displacements as well as rotations resulting in a geometrically nonlinear stress-strain response. Therefore, finite element modeling of this class of materials calls for a geometrically nonlinear formulation. During elastomeric deformation of a polymeric foam, although displacements and rotations of the struts can be quite large, constituent material response itself can still be in the small strain regime. Thus, these struts behave as highly flexible beams with a low bending rigidity. A corotational formulation (Belytschko and Hsieh, 1973; Crisfield, 1991; Belytschko et al., 2000) is particularly suited for this situation. The main idea behind this formulation is to decompose the motion of a body into a rigid body rotation and a pure deformation, akin to polar decomposition of deformation gradient, by introducing a local coordinate system that continuously rotates and translates with the body. In the present study, we model the cellular microstructure as a network of flexible 2D corotational beams thus incorporating finite kinematic effect. Basic features of the corotational beam formulation are described below.

\subsubsection{Beam kinematics}

Rigid rotation must be evaluated accurately in order to find the actual angular deformation at the corotated frames. Earlier methods (Belytschko and Hsieh, 1973;

Crisfield, 1991; Belytschko et al., 2000) restrict the evaluation of such quantity when rigid rotation is extremely high. Hence, a modified approach has been proposed and 
discussed in subsequent section.

\subsubsection{Earlier scheme and its limitation}

The kinematic quantities are evaluated on a local frame attached to the deformed beam. In Figure 2.1, $x-y$ denotes the global coordinate system, whereas $x_{l}-y_{l}$ denotes the local coordinate system at $t=t_{n}$ associated with a two noded beam element. Global

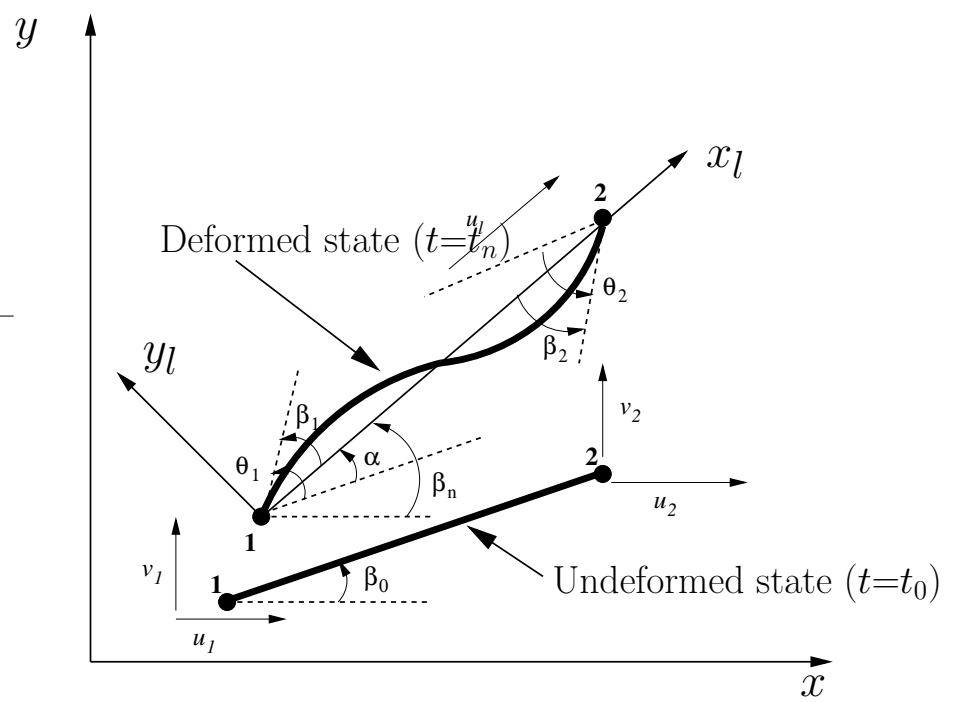

Figure 2.1: Schematic diagram of a $2 \mathrm{D}$ corotational beam element. $x-y$ is the global coordinate system while $x_{l}-y_{l}$ is the rotated local coordinate system in which local deformations are defined.

displacement vector $\mathbf{d}_{g}$ for this element is defined by,

$$
\mathbf{d}_{g}=\left\{\begin{array}{llllll}
u_{1} & v_{1} & \theta_{1} & u_{2} & v_{2} & \theta_{2}
\end{array}\right\}^{T}
$$

where $u_{i}$ and $v_{i}, i=1,2$, are global nodal displacements in $x$ and $y$ directions, respectively, and $\theta_{i}, i=1,2$, are global nodal rotations. Initial and current lengths of the beam element, denoted by $l_{0}$ and $l_{n}$ respectively. Local coordinate axes are so chosen 
that local displacement vector $\mathbf{d}_{l}$ has only three components (Figure 2.1);

$$
\mathbf{d}_{l}=\left\{\begin{array}{lll}
u_{l} & \beta_{1} & \beta_{2}
\end{array}\right\}^{T},
$$

with $u_{l}$ as the axial deformation of the beam and $\beta_{1}$ and $\beta_{2}$ as nodal angular deformations in the corotated coordinate system. Components of the vector $\mathbf{d}_{l}$ can be computed as

$$
\begin{aligned}
& u_{l}=l_{n}-l_{0}, \\
& \beta_{1}=\theta_{1}-\alpha_{n}, \\
& \beta_{2}=\theta_{2}-\alpha_{n},
\end{aligned}
$$

where $\alpha_{n}$ is the rigid body rotation given by

$$
\alpha_{n}=\beta_{n}-\beta_{0} .
$$

In the above equation, $\beta_{n}$ is the current inclination of the local frame with respect to the global coordinate description, and $\beta_{0}$ is the initial inclination of the element (Figure 2.1). The current inclination $\left(\beta_{n}\right)$ of the frame $\left(t=t_{n}\right)$ can be computed as following. The cosine and sine components of the current inclination $\left(\beta_{n}\right)$ are given as

$$
\begin{aligned}
& c^{n}=\cos \beta_{n}=\frac{1}{l_{n}}\left(x_{2}+u_{2}-x_{1}-u_{1}\right), \\
& s^{n}=\sin \beta_{n}=\frac{1}{l_{n}}\left(y_{2}+v_{2}-y_{1}-v_{1}\right) .
\end{aligned}
$$

So the current inclination $\beta_{n}$ can be found by applying quadrant rule as 


$$
\begin{aligned}
& \beta_{n}=\sin ^{-1}\left(s^{n}\right) \quad \text { if } \quad \sin \left(\beta_{n}\right) \geq 0 \quad \text { and } \quad \cos \left(\beta_{n}\right) \geq 0, \\
& \beta_{n}=\cos ^{-1}\left(c^{n}\right) \quad \text { if } \quad \sin \left(\beta_{n}\right) \geq 0 \quad \text { and } \quad \cos \left(\beta_{n}\right)<0, \\
& \beta_{n}=\sin ^{-1}\left(s^{n}\right) \quad \text { if } \quad \sin \left(\beta_{n}\right)<0 \quad \text { and } \quad \cos \left(\beta_{n}\right) \geq 0, \\
& \beta_{n}=-\cos ^{-1}\left(c^{n}\right) \quad \text { if } \quad \sin \left(\beta_{n}\right)<0 \quad \text { and } \quad \cos \left(\beta_{n}\right)<0 .
\end{aligned}
$$

The rotation can be calculated by finding out which quadrant the beam lies. The above relationships hold for only when $\left|\beta_{n}\right|<\pi$.

\subsubsection{Proposed scheme for kinematic quantities}

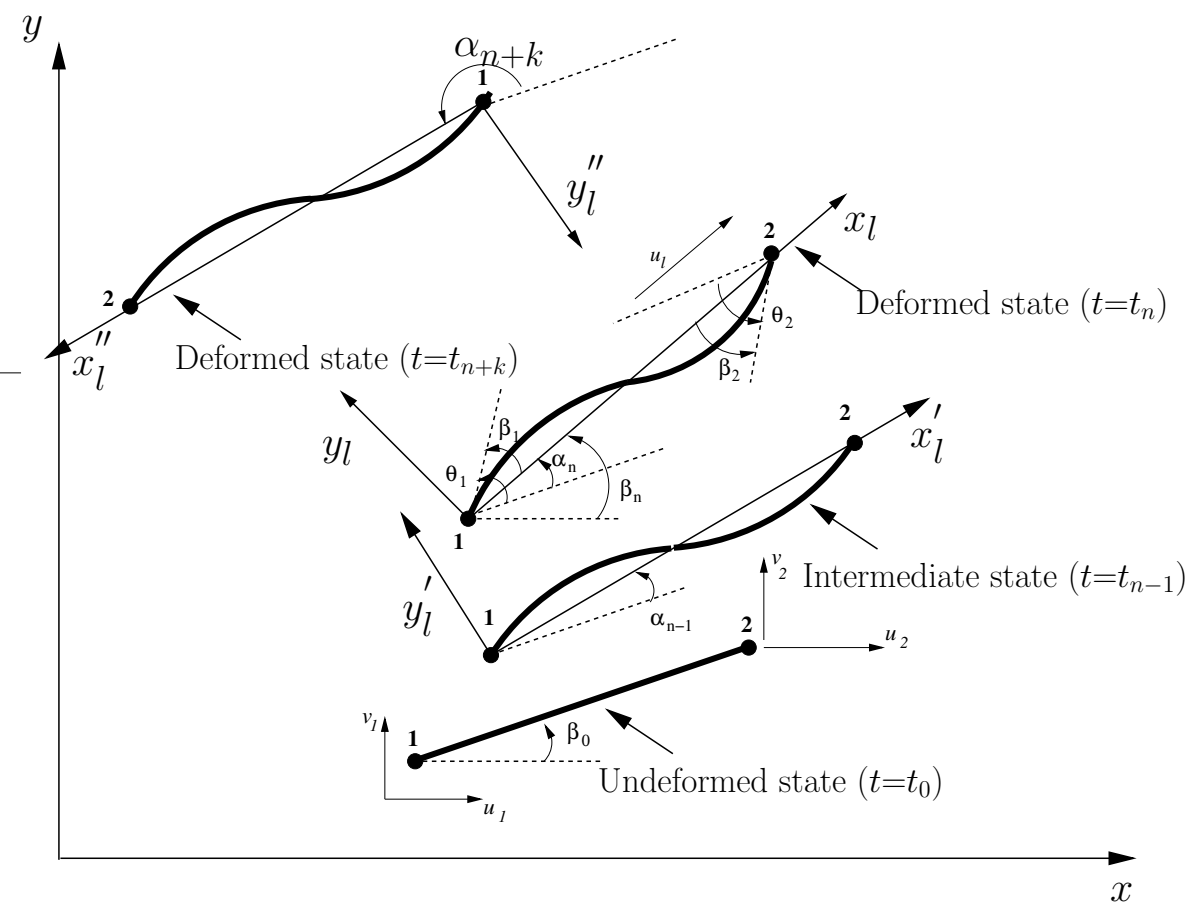

Figure 2.2: Schematic diagram of a $2 \mathrm{D}$ corotational beam element undergoing large rotation. $x-y$ is the global coordinate system while $x_{l}$ - $y_{l}$ is the rotated local coordinate system in which local deformations are defined. $x^{\prime \prime}-y^{\prime \prime}$ denotes local coordinate under large rotation. An intermediate configuration is denoted by $x_{l}^{\prime}-y_{l}^{\prime}$.

In case of soft cellular material, the strut may undergo large rotation $(>\pi)$. The deformed strut at $t=t_{n+k}$ in Figure 2.2 represents such a situation. The earlier approach 
fails to evaluate such a large rotation of the strut. Hence, an alternative approach has been suggested in this dissertation to evaluate the current inclination of the corotated frame. In this novel approach, the inclination at the current configuration $\left(t^{n}\right)$ is evaluated with respect to the previouly known deformed configuration $\left(t^{n-1}\right)$. The change of the inclination at the current state $\Delta \beta_{n}$ from previously deformed state $\left(t^{n-1}\right)$ can be computed as following:

$$
\begin{aligned}
& \cos \left(\Delta \beta_{n}\right)=c^{n} c^{n-1}+s^{n} s^{n-1}, \\
& \sin \left(\Delta \beta_{n}\right)=s^{n} c^{n-1}-c^{n} s^{n-1} .
\end{aligned}
$$

In the above equation $c^{n}$ and $s^{n}$ are cosine and sine components of the current inclination while $c^{n-1}$ and $s^{n-1}$ denote the same for the previous step. These components are evaluated according to Equation 2.5. It can be assumed that change of inclination for any given step is such that $\left|\Delta \beta_{n}\right|<\pi$. Therefore, the quadrant rule can be applied to evaluate $\Delta \beta_{n}$. Hence, the current inclination can be updated as

$$
\beta_{n}=\beta_{n-1}+\Delta \beta_{n}
$$

Thus, the inclination of the frame can be found precisely without facing any numerical difficulties as mentioned earlier. Therefore, the kinematic quantities can be evaluated accurately by extracting the rigid rotation of the corotated frame.

\subsubsection{Calculation of virtual internal work}

The virtual work of the corotational beam in the local and global system can be found as

$$
\delta W_{i n t}=N \delta u_{l}+M_{1} \delta \beta_{1}+M_{2} \delta \beta_{2}=\delta \mathbf{d}_{l}^{T} \mathbf{F}_{l}^{i n t}=\delta \mathbf{d}_{g}^{T} \mathbf{F}_{g}^{i n t}
$$


Local internal force vector, $\mathbf{F}_{l}^{\text {int }}$ has only three components corresponding to the three components of local displacement vector $\mathbf{d}_{l}$ (Equation (2.3)) and can be expressed as

$$
\mathbf{F}_{l}^{\text {int }}=\left[\begin{array}{lll}
N & M_{1} & M_{2}
\end{array}\right]^{T},
$$

where $N$ is the axial load while $M_{1}$ and $M_{2}$ are moments at node 1 and 2 of the element. $\mathbf{F}_{g}^{\text {int }}$ denotes global force vector. Variation of local kinematic quantities $\delta \mathbf{d}_{l}$ can be obtained through the differentiation of Equation (2.3)

$$
\begin{aligned}
\delta u_{l} & =c^{n}\left(\delta u_{2}-\delta u_{1}\right)+s^{n}\left(\delta v_{2}-\delta v_{1}\right), \\
& =\left\{\begin{array}{llllll}
-c^{n} & -s^{n} & 0 & c^{n} & s^{n} & 0
\end{array}\right\}^{T} \delta \mathbf{d}_{l} \\
\delta \beta_{1} & =\delta \theta_{1}-\delta \alpha_{n}=\delta \theta_{1}-\delta \beta_{n}, \\
\delta \beta_{2} & =\delta \theta_{2}-\delta \alpha_{n}=\delta \theta_{2}-\delta \beta_{n} .
\end{aligned}
$$

After mathematical manipulation, variation of current inclination $\delta \beta_{n}$ can be related to global virtual kinematic quantities $\delta \mathbf{d}_{g}$ as

$$
\delta \beta_{n}=\left\{\begin{array}{llllll}
-s^{n} / l_{n} & c^{n} / l_{n} & 0 & s^{n} / l_{n} & -c^{n} / l_{n} & 0
\end{array}\right\}^{T} \delta \mathbf{d}_{g} .
$$

The local virtual kinematic quantity $\delta \mathbf{d}_{l}$ can be related to global quantity as $\delta \mathbf{d}_{l}=$ $\mathbf{B}^{T} \delta \mathbf{d}_{g}$, where the transformation matrix $\mathbf{B}$, can be obtained as

$$
\mathbf{B}=\left\{\begin{array}{cccccc}
-c^{n} & -s^{n} & 0 & c^{n} & s^{n} & 0 \\
-s^{n} / l_{n} & c^{n} / l_{n} & 1 & s^{n} / l_{n} & -c^{n} / l_{n} & 0 \\
-s^{n} / l_{n} & c^{n} / l_{n} & 0 & s^{n} / l_{n} & -c^{n} / l_{n} & 1
\end{array}\right\}
$$

From the Equation (2.9), the global internal load vector can be written as

$$
\mathbf{F}_{g}^{i n t}=\mathbf{B}^{T} \mathbf{F}_{l}^{i n t}
$$


Depending on the beam definition and material constitutive relationship, the local internal force vector $\mathbf{F}_{l}^{\text {int }}$ is calculated and hence the global internal force vector $\mathbf{F}_{g}^{\text {int }}$.

\subsubsection{Euler Bernoulli beam element}

Based on classical linear beam theory, the Euler Bernoulli element is considered here. A linear interpolation for the axial displacement $u$ and a cubic one for vertical displacement $v$ results

$$
\begin{aligned}
& u=\frac{x}{L} u_{l}, \\
& v=x\left(1-\frac{x}{L}\right)^{2} \beta_{1}+\frac{x^{2}}{L}\left(\frac{x}{L}-1\right) \beta_{2} .
\end{aligned}
$$

Considering the assumption of Euler-Bernoulli beam definition the curvature $\kappa$ and strain $\varepsilon$ can be found as

$$
\begin{aligned}
& \kappa=\frac{\partial^{2} v}{\partial x^{2}}=\left(-\frac{4}{L}+6 \frac{x}{L^{2}}\right) \beta_{1}+\left(-\frac{2}{L}+6 \frac{x}{L^{2}}\right) \beta_{2}, \\
& \varepsilon=\frac{\partial u}{\partial x}-\kappa z=\frac{\bar{u}}{L}+z\left[\left(\frac{4}{L}-6 \frac{x}{L^{2}}\right) \beta_{1}+\left(\frac{2}{L}-6 \frac{x}{L^{2}}\right) \beta_{2}\right] .
\end{aligned}
$$

The virtual internal work can be expressed as

$$
\delta W_{i n t}=\int_{\Omega} \sigma \delta \varepsilon d \Omega=N \delta u_{l}+M_{1} \delta \beta_{1}+M_{2} \delta \beta_{2}
$$

where components of $\mathbf{F}_{l}^{\text {int }}$, such as $N, M_{1}$ and $M_{2}$ can be found as

$$
\begin{aligned}
N & =\int_{\Omega} \frac{\sigma}{L} d V \\
M_{1} & =\int_{\Omega} \sigma z\left(\frac{4}{L}-6 \frac{x}{L^{2}}\right) d V, \\
M_{1} & =\int_{\Omega} \sigma z\left(\frac{2}{L}-6 \frac{x}{L^{2}}\right) d V .
\end{aligned}
$$




\subsubsection{Timoshenko beam element}

Timoshenko beam definition (Crisfield, 1991) which will account possible shear deformations of the short cell wall is considered in the local coordinate system. For the two noded element, shape function is given as $\mathbf{N}=\{1-x / L \quad x / L\}$ for the displacement and angular deformation

$$
\begin{aligned}
u & =\frac{x}{L} u_{l}, \\
w & =0, \\
\theta & =N_{1} \beta_{1}+N_{2} \beta_{2},
\end{aligned}
$$

The curvature $\kappa$, shear deformation $\gamma$ and axial strain $\varepsilon$ can be obtained as

$$
\begin{aligned}
\kappa & =\frac{\partial \theta}{\partial x}=\frac{\beta_{2}-\beta_{1}}{l_{n}}, \\
\gamma & =\frac{\partial w}{\partial x}-\theta=-N_{1} \beta_{1}-N_{2} \beta_{2}, \\
\varepsilon & =\frac{\partial u}{\partial x}-\kappa z=\frac{u_{l}}{l_{n}}-\frac{\beta_{2}-\beta_{1}}{l_{n}} z .
\end{aligned}
$$

The virtual internal work can be expressed as

$$
\delta W_{i n t}=\int_{\Omega}(\sigma \delta \varepsilon+\tau \delta \gamma) d \Omega=N \delta u_{l}+M_{1} \delta \beta_{1}+M_{2} \delta \beta_{2}
$$

where $N, M_{1}$ and $M_{2}$ can be obtained as

$$
\begin{aligned}
N & =\int_{\Omega} \frac{\sigma}{L} d V \\
M_{1} & =\int_{\Omega}\left[\frac{\sigma}{L} z-\tau\left(1-\frac{x}{L}\right)\right] d V, \\
M_{2} & =\int_{\Omega}\left[-\frac{\sigma}{L} z-\tau \frac{x}{L}\right] d V .
\end{aligned}
$$


In the above equation $\sigma$ and $\tau$ are axial and shear stresses in the beam, respectively, while $x \in[0 L]$, and $z \in\left[-h_{s} / 2 h_{s} / 2\right]$ with $h_{s}$ as the beam thickness.

\subsubsection{Calculation of axial force and moments}

Typically a Gauss-Quadrature rule is used to evaluate the axial force as well as moments as given in Equation (2.18) and (2.22). In case of inelastic materials, a discontinuous stress distribution arises across the depth as shown in the Figure 2.3. Gauss-Quadrature is not suitable for inelastic material since the quadrature schemes are based on higher order polynomial which by default assume the smoothness in the data under consideration. The trapezoidal rule is more suitable as it is more effective for less smooth function. Assuming a rectangular cross-section beam with thickness
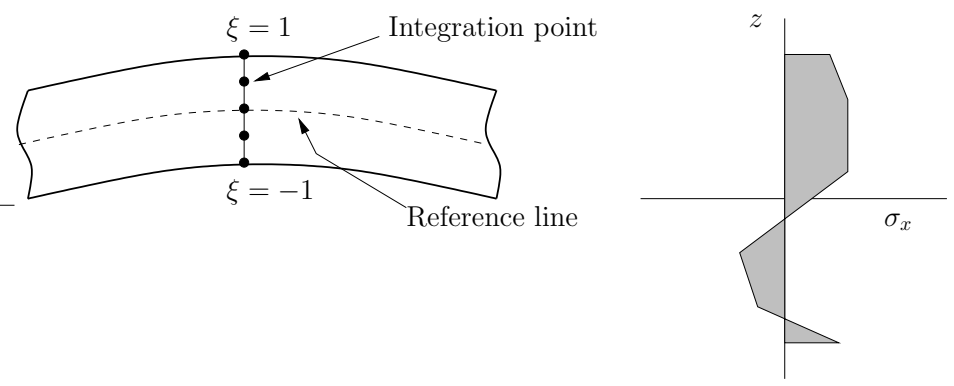

Figure 2.3: Stress distribution across the depth of the beam when constitutive material is inelastic.

$h_{s}$ and width $b$, axial force can be evaluated as

$$
N=\int_{-h_{s} / 2}^{h_{s} / 2} \sigma(z) d A=\int_{-1}^{1} \sigma(\xi) J b d \xi
$$

where $\xi=2 z / h_{s}$, and $J=d z / d \xi$. The moment $M_{1}$ in Equation (2.22) can be evaluated as

$$
M_{1}=\int_{-h_{s} / 2}^{h_{s} / 2} \sigma(z) z d A-\frac{l_{0}}{2} \int_{-h_{s} / 2}^{h_{s} / 2} \tau(z) d A=\int_{-1}^{1} f(\xi) J b d \xi-\frac{l_{0}}{2} \int_{-1}^{1} \tau(\xi) J b d \xi
$$


where $f(\xi)=\sigma(\xi) h_{s} \xi / 2$. The moment $M_{2}$ can be evaluated in similar manner. The detail steps to evaluate the stress for a rate independent plastic material is described in Appendix C.

\subsection{Explicit transient finite elements for the beam element}

A transient finite element method with an explicit time stepping scheme is used to simulate the dynamic response of the soft cellular solids. Condition for equilibration at the element level can be derived from the principle of virtual work, and can be stated as

$$
\delta W_{k i n}+\delta W_{i n t}-\delta W_{e x t}=\mathbf{0}
$$

where the variation of kinetic energy, internal energy and external work done can be written as

$$
\begin{aligned}
\delta W_{k i n} & =\int_{\Omega} \delta \mathbf{u} \cdot \rho \ddot{\mathbf{u}} d V+\int_{\Omega} \delta \boldsymbol{\theta} \cdot I \ddot{\boldsymbol{\theta}} d V=\delta \mathbf{d}_{g}^{T} \mathbf{M} \ddot{\mathbf{d}}_{g}, \\
\delta W_{i n t} & =\delta \mathbf{d}_{g}^{T} \mathbf{F}_{g}^{i n} \\
\delta W_{\text {ext }} & =\delta \mathbf{d}_{g}^{T} \mathbf{F}_{g}^{e x t}
\end{aligned}
$$

Substituting individual virtual quantities in Equation (2.25), a discretized equation of motion can be obtained as

$$
\mathbf{M} \ddot{\mathbf{d}}_{g}=\mathbf{F}_{g}^{e x t}-\mathbf{F}_{g}^{i n t}
$$

Mass matrix $\mathbf{M}$ in the above equation depends on the element description and mass density $\rho$. We have used a lumped mass matrix using HRZ lumping scheme (Cook et al., 
2002). $\mathbf{F}_{g}^{e x t}$ denotes the external load vector while internal load vector $\mathbf{F}_{g}^{\text {int }}$ can be evaluated from Equation (2.14). Equations of motion (2.27) are now integrated explicitly in time domain using a central difference scheme (Belytschko et al., 1976) to compute global velocity $\dot{\mathbf{d}}_{g}$ and displacement $\mathbf{d}_{g}$ as

$$
\begin{aligned}
& \dot{\mathbf{d}}_{g}^{n+1}=\dot{\mathbf{d}}_{g}{ }^{n}+\frac{1}{2} \Delta t\left[\ddot{\mathbf{d}}_{g}{ }^{n}+\ddot{\mathbf{d}}_{g}{ }^{n+1}\right] \\
& \mathbf{d}_{g}{ }^{n+1}=\mathbf{d}_{g}{ }^{n}+\frac{1}{2} \Delta t \dot{\mathbf{d}}_{g}{ }^{n}+\frac{1}{2} \Delta t^{2} \ddot{\mathbf{d}}_{g}{ }^{n}
\end{aligned}
$$

where $(\cdot)^{n+1}$ represents the quantities at time step $t=t^{n+1}$ while $(\cdot)^{n}$ denotes same quantities at time step $t=t^{n}$.

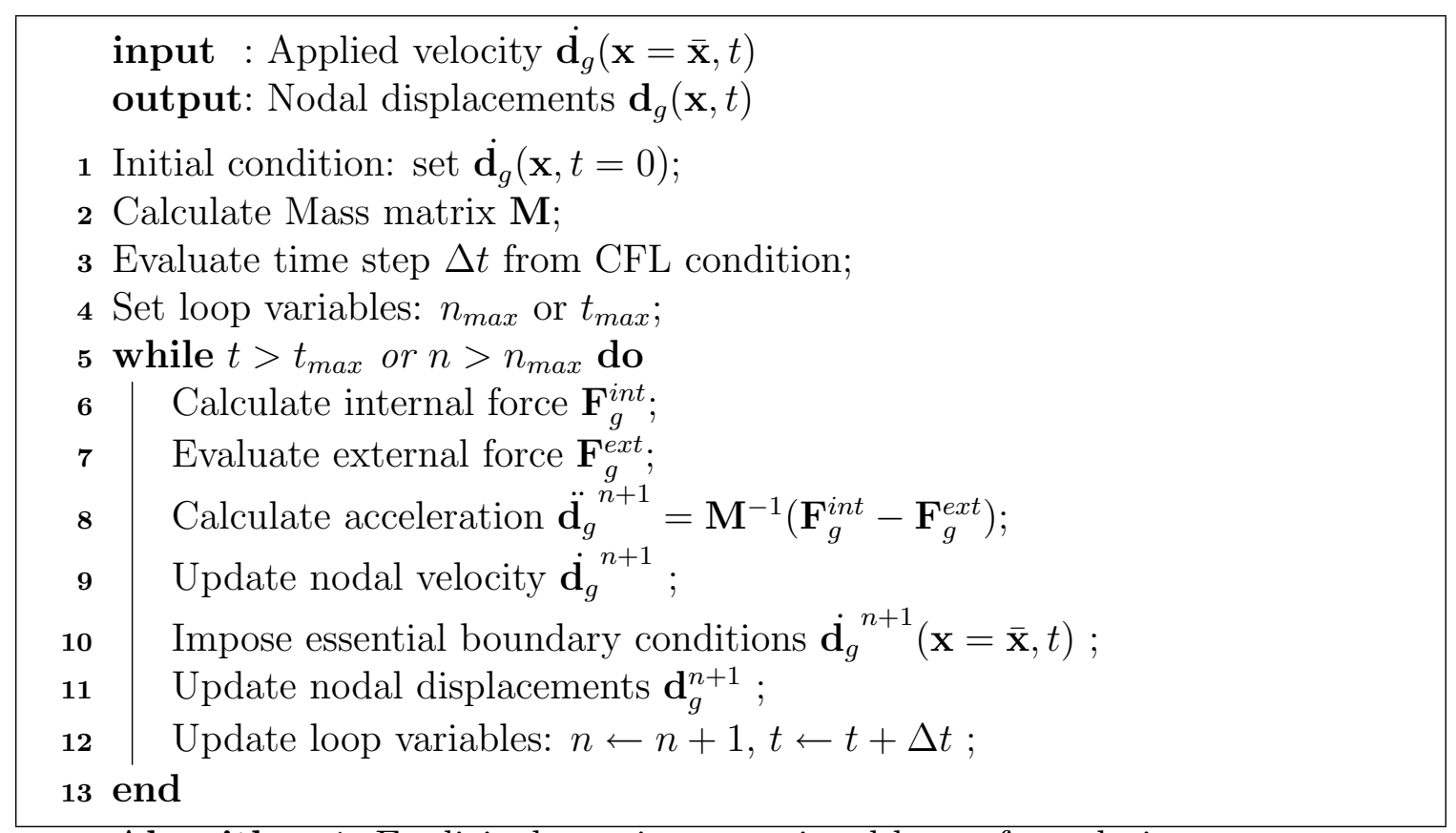

Algorithm 1: Explicit dynamic corotational beam formulation.

Explicit time stepping algorithms are conditionally stable such that time step size must be less than a critical time step size. For example, if a wave is travelling in an element, then the critical time step is equal to the time for wave to travel from one end to another end of the element. The time step size for the explicit time-marching 
schemes is determined by Courant-Friedrichs-Lewy (CFL) condition as

$$
\Delta t=\psi \frac{\Delta x}{c_{d}}
$$

where $\psi$ is the Courant number, $\Delta x$ is the minimum element length and $c_{d}$ is the dilatational wave speed in the element. For the simulations presented in this paper, we have chosen $\psi=0.1$ so that the solutions remain well within the stable regime. Numerical implementation of explicit dynamic corotational formulation can be performed through Algorithm 1.

\subsection{Implicit quasi-static finite element corotational beam formulation}

To find the quasi-static response of the flexible beam an implicit corotational beam formulation has been developed.

$$
\mathbf{R}\left(\delta \mathbf{d}_{g}\right):=\delta W_{i n t}-\delta W_{e x t}=\mathbf{0}
$$

where, $\mathbf{R}$ defined as residual vector. The above equation can be linearized for a given increment of global displacement vector $\Delta \mathbf{d}_{g}$ as

$$
\mathbf{R}\left(\mathbf{d}_{g}+\Delta \mathbf{d}_{g}\right)=\mathbf{R}\left(\mathbf{d}_{g}\right)+\frac{\partial \mathbf{R}}{\partial \mathbf{d}_{g}} \Delta \mathbf{d}_{g} \approx \mathbf{0}
$$

Using Equation (2.30) in above equation, the linearized equations can be restated as

$$
\mathbf{K}_{g}^{i} \Delta \mathbf{d}_{g}^{i+1}=\mathbf{F}_{g}^{e x t^{i}}-\mathbf{F}_{g}^{i n t^{i}}
$$


where tangent stiffness matrix $\mathbf{K}_{g}=\partial \mathbf{R} / \partial \mathbf{d}_{g}=\partial \delta W_{\text {int }} / \partial \mathbf{d}_{g}$. In the above equation $\mathbf{F}_{g}^{\text {int }}$ is the internal load vector can be evaluated from corotational beam definition as given in Equation (2.14) along with correct beam definition. An iterative procedure is applied to solve Equation (2.32) where $i$ denotes the index of iteration. For a given load step $(n)$, the displacement vector is updated as

$$
\mathbf{d}_{g}^{i+1}=\mathbf{d}_{g}^{i}+\Delta \mathbf{d}_{g}^{i+1},
$$

and the iteration continues until increment of displacement reaches a convergence criteria specified as $\left\|\Delta \mathbf{d}_{g}^{i+1}\right\|_{L_{2}}<\epsilon\left\|\mathbf{d}_{g}^{i}\right\|_{L_{2}}$, where $\epsilon$ is the tolerance. Numerical implementation of implicit corotational formulation for quasi-static analysis can be performed through Algorithm 2.

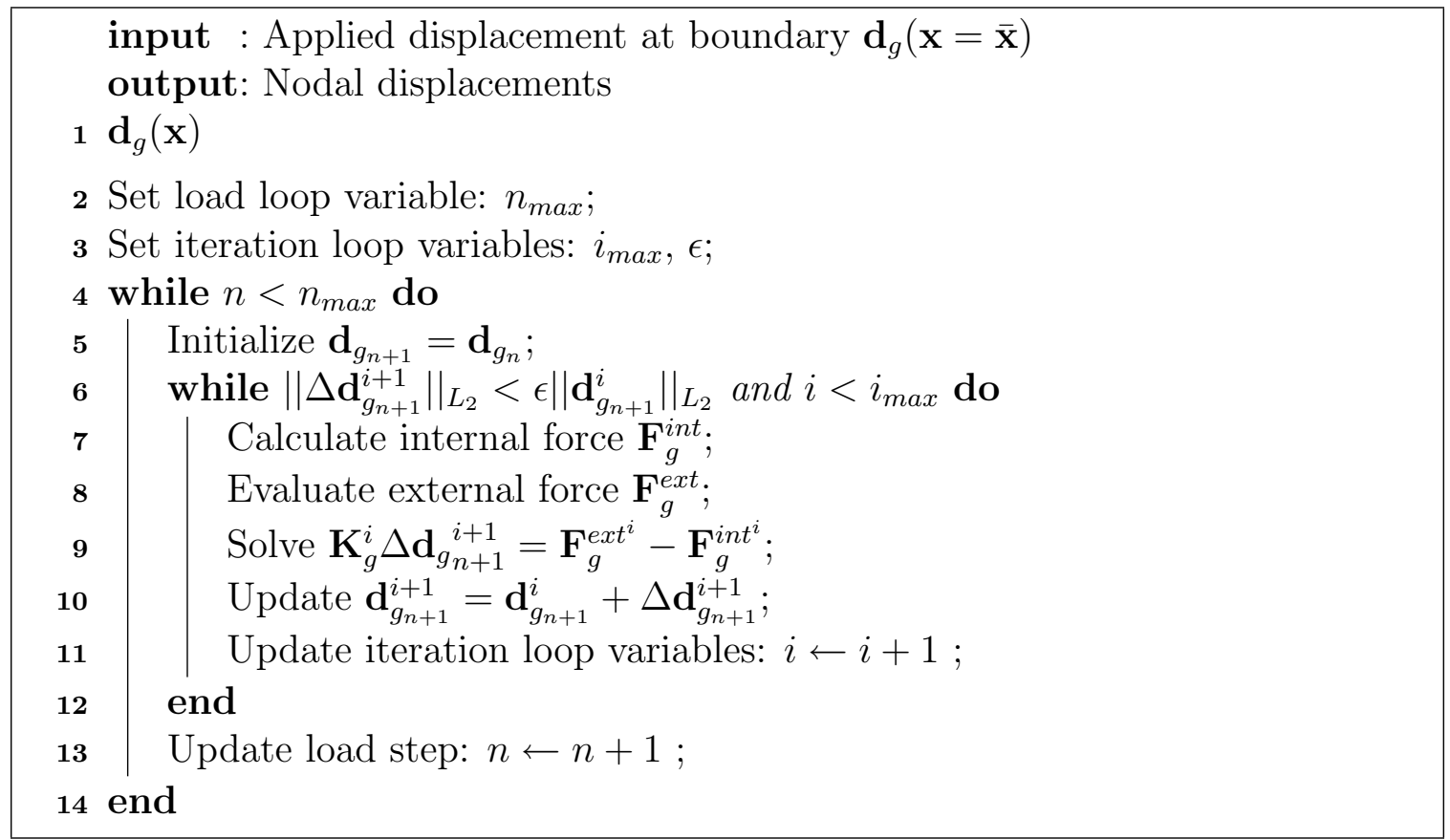

Algorithm 2: Implicit quasi-static corotational beam formulation. 


\subsection{Simulation for the verification of developed co- mputational scheme.}

\subsubsection{Deflection of elastic cantilever beam under impact load: Theory and numerical experiment}

A cantilever beam of uniform cross-section as shown in Figure 2.4 subjected to a transient force $\operatorname{Pf}(t)$ at the tip is considered for the verification purpose. The force $P f(t)$ is a half-sine pulse as shown in Figure 2.5(a)

$$
f(t)= \begin{cases}\sin \pi t / T & 0 \leq t \leq T \\ 0 & t>T\end{cases}
$$

where $T$ is the period of the fundamental mode of vibration of the cantilever. According to (Warburton, 1976) the tip deflection can be given as

$$
v(l, t)= \begin{cases}\frac{P l^{3}}{E I}[0.441 \sin \pi t / T-0.216 \sin 2 \pi t / T], & 0 \leq t \leq T \\ \frac{P l^{3}}{E I}\left[-0.432 \sin \left(\omega_{1}(t-T)\right)\right], & t>T,\end{cases}
$$

To simulate the above problem, the beam is discretized with two noded EulerBernoulli corotational beam element. Using the explicit dynamic formulation, the tip deflection is obtained and plotted against the analytical solution in Figure 2.5(b) for different number of discretization $(N)$. It can be observed that simulated response is similar to the analytical results. Furthermore, as the number of element increases, the simulated responses converges to the analytical solution as given in Equation (2.34). Thus, the above explicit dynamic corotational formulation is vali- 
dated and can be used as predictive tool to simulate dynamic behavior of complex structures containing networks of beams such as open cell cellular solids.

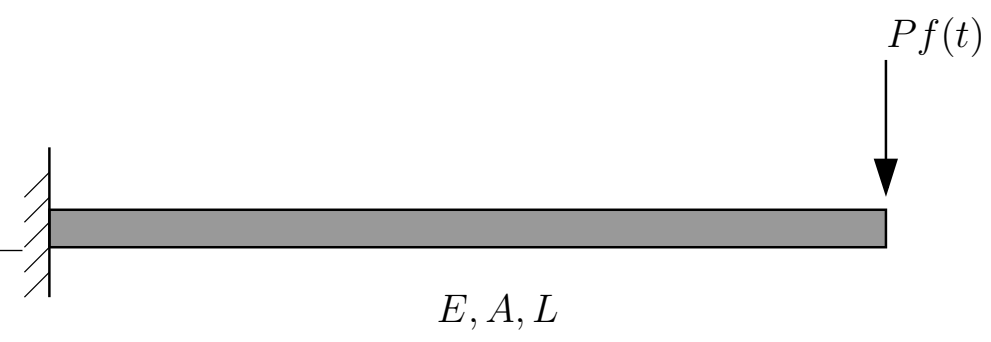

Figure 2.4: Cantilever beam subjected to transient force at the tip.

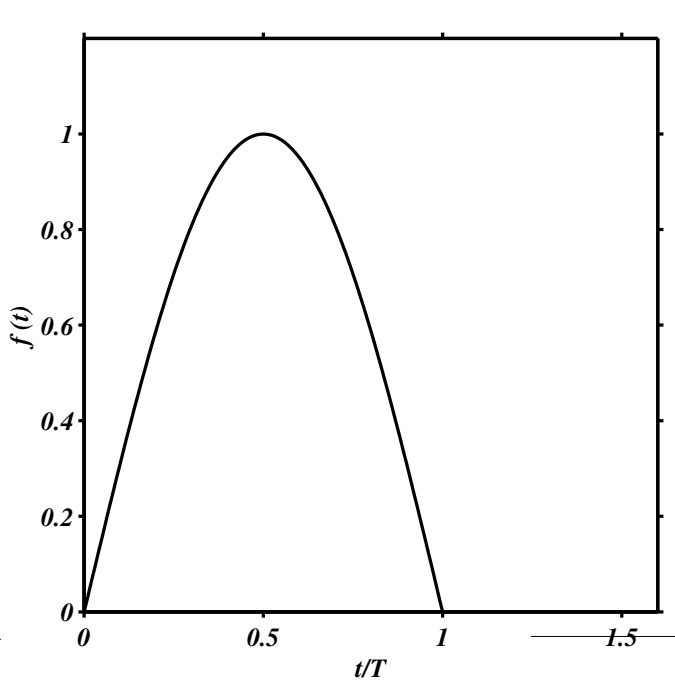

(a)

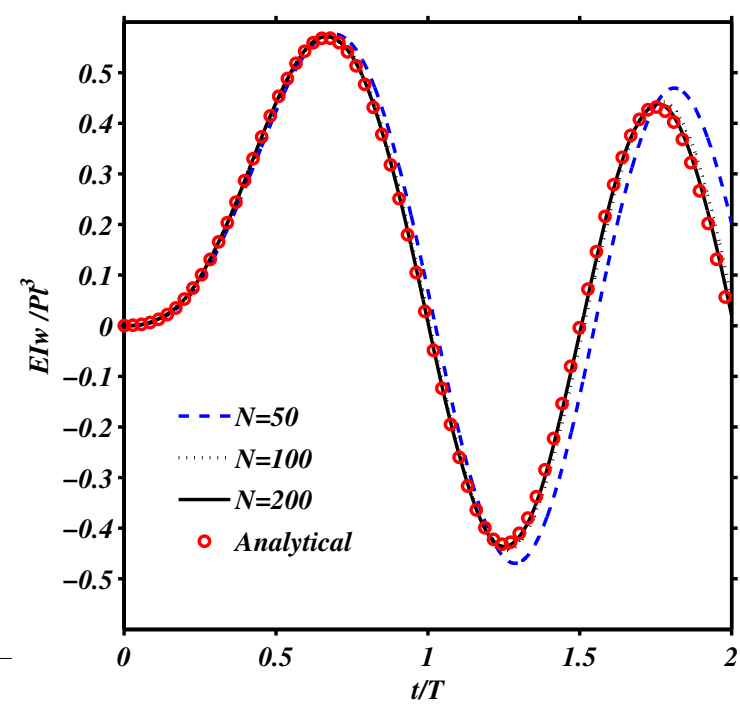

(b)

Figure 2.5: (a) Variation of impact force at the tip with time, and (b) Variation of tip deflection with time.

\subsubsection{Deformation of a crossed bar under impact load: demon- stration of dynamic buckling}

In this example, a crossed bar as shown in Figure 2.6 is subjected to axial impact at free end with velocity $v$. Constituent material of the crossed bar taken as lin- 
early elastic. The beam is discretized with Timoshenko beam elements. Using the explicit dynamic corotational formulation, the deformation snaps at different times are obtained and illustrate and plotted in Figure. 2.7. At the initial stage $t=4.5 \mu s$,

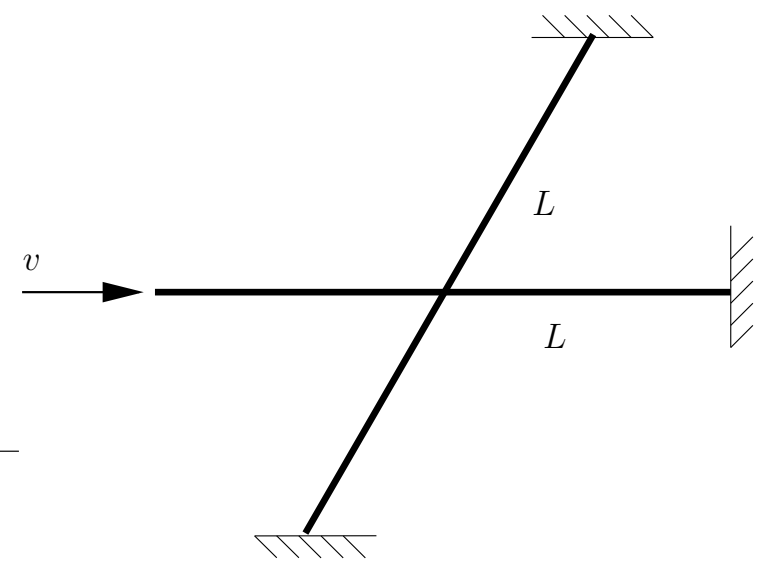

Figure 2.6: Schematic of crossed bar undergoing axial impact at the free end.

the horizontal beam undergoes axial compression while the inclined beam undergoes bending deformation. As time proceeds, $t=7.0 \mu s$ the horizontal beam further undergoes axial deformation with little bending while the inclined beam undergoes significant bending deformation. The large deformation of inclined beam and the axial deformation of the right horizontal segment offer significant resistance at the strut junction. Hence, no further movement of the junction point is possible due to the application of impact velocity. Consequently, at $t=9.5 \mu \mathrm{s}$, the horizontal beam undergoes significant amount of buckling to accommodate the applied displacement. Such crossed bar can roughly be thought the building blocks of open cell cellular material and this deformation characteristics is important for the macroscopic behavior of cellular materials. 


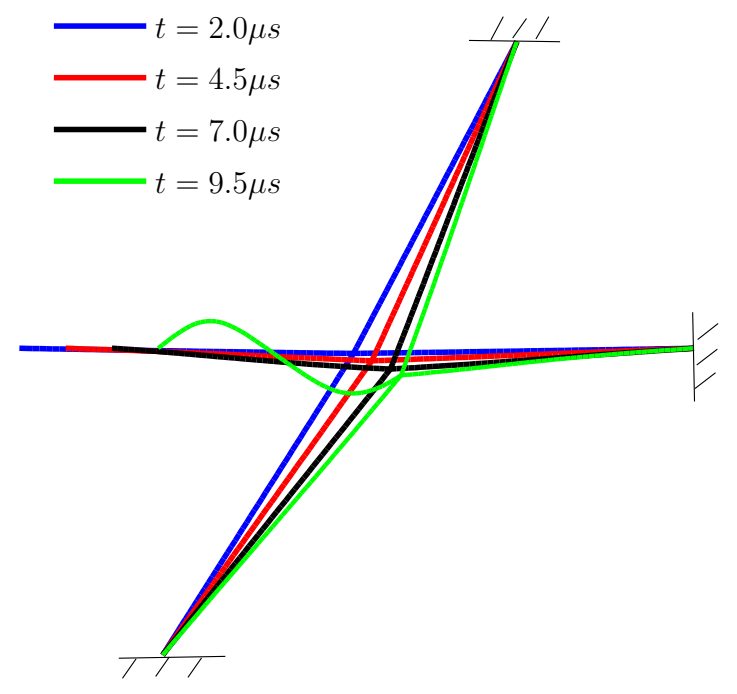

Figure 2.7: Deformed snapshots of the crossed bar when subjected to axial impact at the free end.

\subsection{Cellular microstructure generation \& random- ness quantification}

An accurate representation of the cellular microstructure is necessary to investigate the effect of cellular topological features as well as material properties on deformation characteristics. We employ Voronoi tessellation, a widely used cellular microstructure generation technique having space filling properties similar to the actual foam, for the purpose of our simulations. In the following we will describe the properties of voronoi tessellation, and its implementation to cellular microstructure generation. 


\subsubsection{Voronoi tessellation}

Voronoi tessellation is the decomposition of a space with $n$ points to convex polygons. Let $P=\left\{p_{1}, p_{2}, \ldots, p_{n}\right\}$ be a set of points in the two dimensional Euclidean plane referred to as sites. The voronoi region $V\left(p_{i}\right)$ of site $p_{i}$ is defined as

$$
V\left(p_{i}\right)=\left\{x:\left|p_{i}-x\right| \leq\left|p_{j}-x\right|, \forall j \neq i\right\}
$$

The Voronoi region $V\left(p_{i}\right)$ thus consists all points that are nearer to site $p_{i}$ than any other site $p_{j}$. The set of all points having more than one nearest neighbor constitutes the Voronoi diagram $\mathbb{V}(P)$ of set $P$ (Figure 2.8). It can also be shown that for $n$ sites, the number of Voronoi vertices and edges is $O(n)$. Let us assume the perpendicular bisector of the line segment $p_{i} p_{j}$ by $B_{i j}$. Also, let $H\left(p_{i}, p_{j}\right)$ be a closed half-plane with boundary $B_{i j}$ and containing the site $p_{i}$. It can be shown that

$$
\mathbb{V}\left(p_{i}\right)=\bigcap_{i \neq j} H\left(p_{i}, p_{j}\right)
$$

Note that the voronoi regions are always convex and contain more than three edges.

\subsubsection{Numerical implementation of cellular microstructure generation}

Voronoi tessellation technique has been implemented to generate cellular microstructures. Voronoi seeds, when distributed on a regular triangular lattice, result in a honeycomb cellular structure. Disorder in this regular structure can be simulated by introducing a random perturbation to the seed positions. The detail steps to generate cellular microstructure is described in Appendix B. Microstructures thus generated 


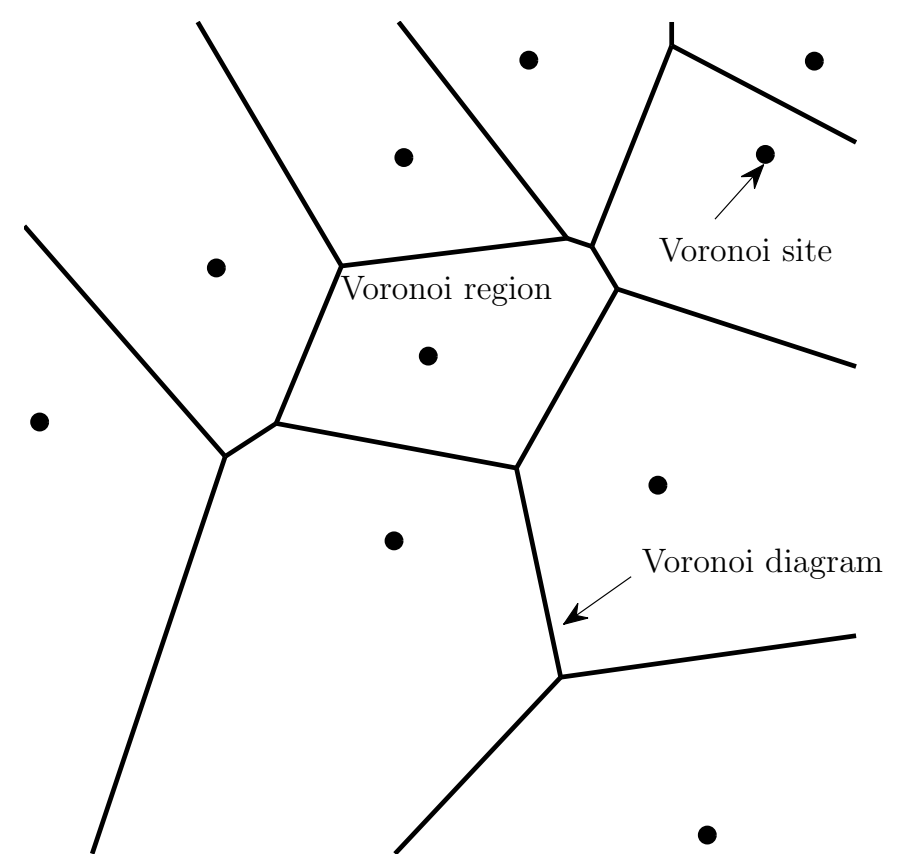

Figure 2.8: Voronoi tessellation showing Voronoi sites, Voronoi regions and a Voronoi diagram.

are characterized by the mean cell size $d_{a v}$ and standard deviation $S_{d}$ of the cell size distribution (Figure 2.9(b)). In the present analysis, we will consider the randomness only in the microstructure. With a uniform strut thickness of $h_{s}$ for all the cells, relative density of a cellular structure can be defined as

$$
\bar{\rho}=\frac{\rho}{\rho_{s}}=\frac{\sum_{i=1}^{n_{s}} l_{i} \kappa h_{s}^{2}}{L D h_{s}}
$$

where $\rho$ is the apparent density of cellular structure, $\rho_{s}$ is the bulk material density of constituent material of the cellular specimen, $n_{s}$ is the number of struts, $l_{i}$ are the length of struts, and $\kappa$ is a dimensionless parameter depending upon the shape of the strut cross-section. Each strut (cell wall) is further subdivided into a number of two noded beam elements to perform the finite element analysis described next. 


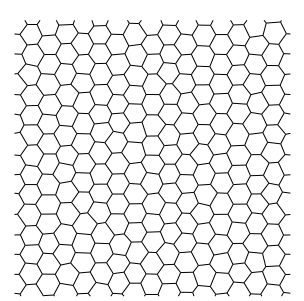

(a)

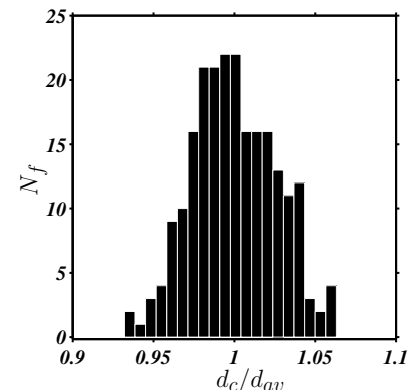

(b)

Figure 2.9: (a) Numerically generated random open cellular structure with an average cell size $d_{a v}=28.4 \mu \mathrm{m}$ and standard deviation $S_{d}=0.02 d_{a v}$, and (b) resulting cell size distribution normalized with $d_{a v}$. Frequency of occurrence of a particular cell size is denoted by $N_{f}$, while $d_{c}$ stands for the size of an individual cell.

\subsection{Experimental validation of foam dynamic re- sponse}

For the validation purpose, a cellular microstructure with length $L=2 \mathrm{~mm}$, thickness $D=2 \mathrm{~mm}$, average cell size $d_{a v}=151 \mu \mathrm{m}$ and struct thickness $h_{s}=14 \mu \mathrm{m}$ is generated. The bulk material is considered as polyvinyl chloride (PVC) whose Young's modulus $E=3.0 \mathrm{GPa}$, and density $\rho=1400 \mathrm{Kg} / \mathrm{m}^{3}$. Using transient explicit corotational formulation, the dynamic response of the cellular specimen is obtained and compared with experimental response (Thomas et al., 2002). It should be noted that, due to extreme variabilities in microstructure and microscopic properties, an exact match with the experiment is impossible to simulate. Our objective is to examine whether the numerical framework developed can capture the experimental outcome in an average sense, and salient features of experimental results are well predicted.

It can be observed from Figure 2.10 that simulated response offers almost similar deformation behavior. Initial slope, peak stress as well as plateau region are predicted well. For example, experimentally observed average plateau stress is $3.4 M P a$, while 


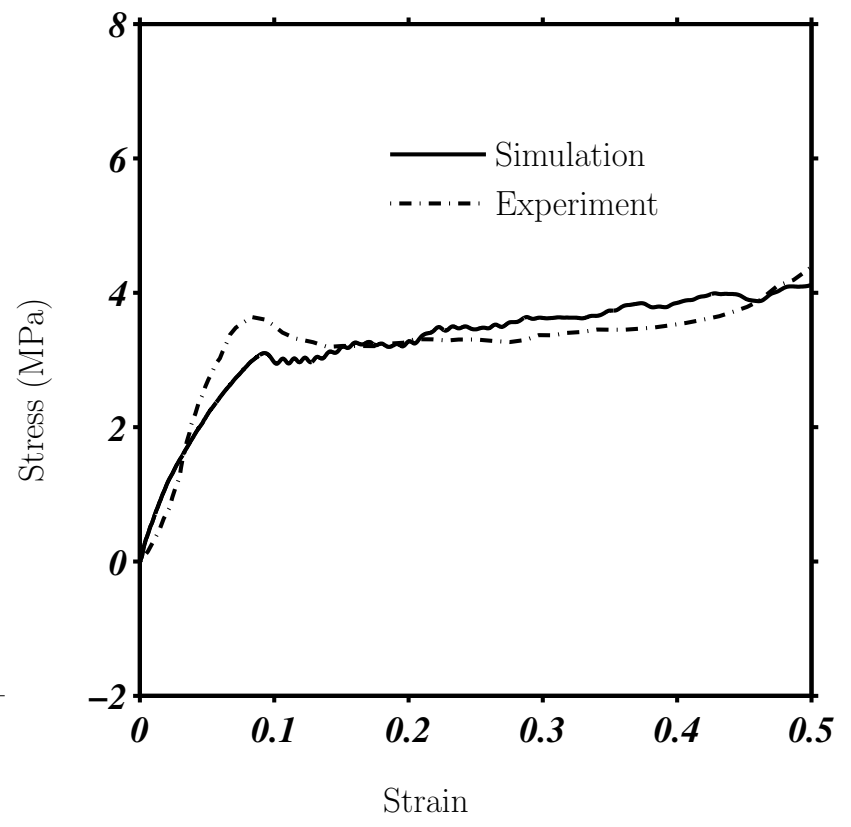

Figure 2.10: Comparison of simulated and experimental responses of a cellular specimen.

numerical simulation offers an average pleatue stress 3.6 $M P a$. Relative error in such prediction is $5.8 \%$. The discrepancies between responses can be attributed from the microstructure variabilities that has not been taken into account.

\subsection{Concluding remarks}

In this chapter, detail steps of corotational beam formulation have been outlined. A new approach has been proposed to avoid the numerical difficulties arising from the large rotation. An explicit dynamic finite element has been implemented to simulate the dynamic behavior of a flexible beam. The nodal internal load is evaluated based on corotational beam formulation. Hence, an accurate nodal force can be calculated though the strut undergoes large rotation. An implicit formulation has also been illustrated to obtain the quasi-static behavior of the flexible elements. The numerical framework is validated against available analytical results. Furthermore, deforma- 
tion of crossed beam is obtained for dynamic impact load. It is found that numerical framework is capable to simulate complex strut subjected to axial load. Therefore, the explicit corotational beam formulation will enable us to study complex deformation characteristic of cellular specimen. Furthermore, using the implicit corotational beam formulation, the deformation behavior of tough biological filament will be obtained. Next, the detail procedures to generate cellular microstructure have been illustrated. The randomness of cellular microstructure have also been characterized through the distribution of cell sizes. Moreover, dynamic response of numerically generated cellular structure has been simulated and compared with experimental observation. 


\section{Chapter 3}

\section{Analysis of stress state in soft cellular materials during split Hopkinson pressure bar (SHPB) test}

\subsection{Introduction}

The developed computational framework has been utilized to study the stress state in a soft cellular sample during SHPB test. Moderately randomized cellular microstructures with different average cell size have been generated computationally to represent the specimen. In order to simulate dynamic test conditions under SHPB setup, a parametric study encompassing intrinsic variables (e.g., specimen aspect ratio and cellular microstructural features) and extrinsic variables (e.g., loading rate and boundary impedance mismatch) has been undertaken. The focus of the present 
work is to study the effect of specimen thickness and impedance mismatch on the dynamic response of soft polymeric cellular materials using the split Hopkinson pressure bar technique. State of stress (equilibrium or non-equilibrium) will be studied thoroughly for a set of geometric and material parameters.

This chapter is organized as follows: In Section 3.2, the computational domain along with boundary conditions is described. Parameters used to characterize the cellular microstructure and describe the dynamic equilibration process are also detailed in this section. In Section 3.3, we present a thorough discussion of a parametric study obtained through the computational framework outlined in Chapter 2. Of special interest is the effect of microstructural features and thickness of the specimen, loading rate, and end impedance mismatch on the dynamic equilibration process. Finally, we close the discussion by drawing conclusions from the current study in Section 3.4.

\subsection{Problem description}

\subsubsection{Setup of the computational model}

In the current study, we mimic SHPB test setup as closely as possible by using a computational domain representing the specimen with proper boundary conditions. Figure 3.1 shows a cellular specimen of length $L$ and depth $D$ with an aspect ratio $\phi=L / D$ held between an incident bar to the left and a transmission bar to the right. Stress wave enters the specimen through the incident bar interface (left) and propagates towards the transmission bar interface (right). Incident and transmitted signals are collected at respective interfaces, and the axial strain in the specimen is measured from the relative displacement of these interfaces. Note that a certain portion of the incident wave gets reflected from the incident bar-specimen interface while the same 


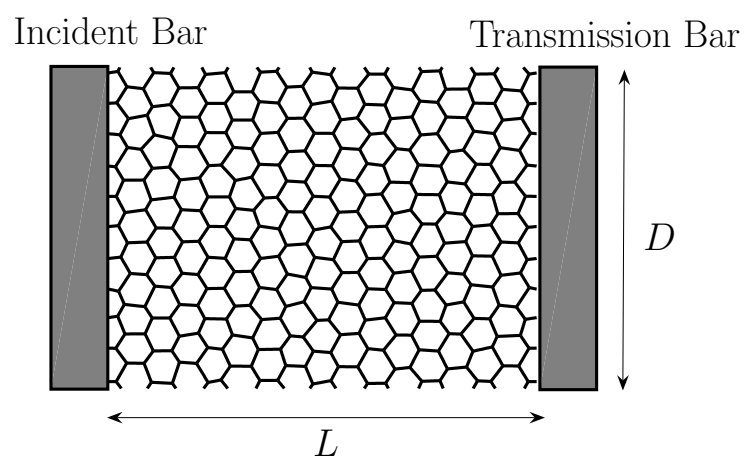

Figure 3.1: A cellular specimen of length $L\left(=13.2 d_{a v}\right)$ and depth $D$ is held between incident and transmission bars of a SHPB set up. Impedance (viscous) boundary conditions are used at left and right ends of the specimen while top and bottom boundaries are kept free.

happens also at the transmission bar-specimen interface due to an impedance mismatch between the specimen and the bars. For a realistic computational simulation of the SHPB technique, this impedance mismatch has to be taken into account.

The incident and transmission bars are replaced by equivalent boundary condition in our computational framework. For this purpose, we have incorporated viscous boundary conditions on the left as well as on the right ends. Viscous dampers have been attached to all the struts at the incident and transmission interfaces with the specimen. Force exerted by such viscous dampers for an interface velocity $v$ can be related as $F_{v}=\rho_{m} c_{m} A_{m} v$, with $\rho_{m}, c_{m}$ and $A_{m}$ are the bulk material density, the dialatational wave speed and cross-sectional area of the external medium, respectively. Impedance mismatch ratio of each strut at the incident bar-specimen interface $\bar{Z}_{I}$ and the transmission bar-specimen interface $\bar{Z}_{T}$ are defined as

$$
\bar{Z}_{I}=\frac{\rho_{I} c_{I} A_{I}}{\rho_{s} c_{d} A}, \quad \bar{Z}_{T}=\frac{\rho_{T} c_{T} A_{T}}{\rho_{s} c_{d} A}
$$

with $\rho_{s}$ as the mass density of bulk cellular material and $c_{d}$ as the dialatational wave 
speed. Also, $\rho_{I}, c_{I}$ are the mass density and dilatational wave speed of the incident bar, whereas, $\rho_{T}, c_{T}$ represent the same quantities at the transmission end. Additionally, $A$ denotes the area of the specimen while $A_{I}$ and $A_{T}$ are cross-sectional area of the incident and transmission bars, respectively. While describing a cellular material, it should be noted that relative density $\bar{\rho}$ of the material is a better parameter to quantify the impedance of the specimen. But $\bar{\rho}$ is microstructure dependent and may vary as the deformation progresses in a cellular solid. So, we have chosen the quantity $\rho_{s}$, actual bulk density of the specimen, instead of $\bar{\rho}$ to avoid this microstructure dependence in the description of the impedance mismatch.

\subsubsection{Extraction of dynamic stress-strain response}

In this computational setup, time dependent stress at the incident end $\sigma_{I}(t)$ and transmission end $\sigma_{T}(t)$ can be found by computing reactions at corresponding ends. Homogenized state of deformation at which dynamic properties of the specimen should be evaluated can be characterized by a measure of dynamic stress equilibration. Using the method of characteristics, Ravichandran and Subhash (1994) have analyzed stress equilibration in a ceramic specimen while being loaded in SHPB. They established a dynamic stress equilibration measure as

$$
R(t)=\frac{|\Delta \sigma(t)|}{\left|\sigma_{m}(t)\right|} \times 100 \%
$$

where $\Delta \sigma(t)=\sigma_{T}(t)-\sigma_{I}(t)$ is the difference in stresses between transmission and incident interfaces and $\sigma_{m}(t)=\left(\sigma_{T}(t)+\sigma_{I}(t)\right) / 2$ is the mean of these values. Stress equilibrium of the specimen is assumed to have been achieved when $R(t) \leq 5 \%$ for ceramic specimens. After ensuring the stress equilibration, the dynamic stress and 
strain can be evaluated in a SHPB setup as

$$
\dot{\varepsilon}=-\frac{2 c_{d}}{L} \varepsilon_{r}(t), \quad \text { and } \quad \sigma(t)=\frac{A_{0}}{A_{s}} E \varepsilon_{t}(t) .
$$

In the above equations, $L$ and $A_{s}$ are the original sample length and cross-sectional area; $c_{d}, E$ and $A_{0}$ are the dilatational wave speed, Young's modulus and crosssectional area of the bars, respectively; $\varepsilon_{r}(t)$ and $\varepsilon_{t}(t)$ are measured reflected and transmitted strain signals. To evaluate the dynamic stress-strain response analogous to SHPB setup, the strain rate of the specimen in the present framework is computed as

$$
\dot{\varepsilon}(t)=\frac{v_{T}-v_{I}}{L},
$$

where $v_{T}$ and $v_{I}$ are the velocities of the interfaces at the transmission and the incident ends, respectively. It can be shown that SHPB relationship 3.3(a) can be deduced from Equation (3.4). Now, axial strain $\varepsilon$ can be obtained as

$$
\varepsilon(t)=\int_{0}^{t} \dot{\varepsilon}(t) d t .
$$

Our numerical model can provide the transmitted stress $\sigma_{T}(t)$ directly, measured at the specimen and transmission bar interface.

\subsubsection{Theoretical verification}

In order to verify the proposed computational scheme, our simulation results for a one-dimensional specimen are compared to the results for the stress equilibration in a typical ceramic specimen. Impedance mismatch at both the incident bar-specimen and the transmission bar-specimen interfaces has been kept same, i.e. $\bar{Z}=\bar{Z}_{I}=\bar{Z}_{T}$. Using our scheme, the stress equilibration curves of an AlN specimen ( $E=315 G P a$ and $\rho_{s}=3226 \mathrm{Kg}^{-3}$ ) with a length of $9.52 \mathrm{~mm}$ subjected to different $\bar{Z}$ are ob- 


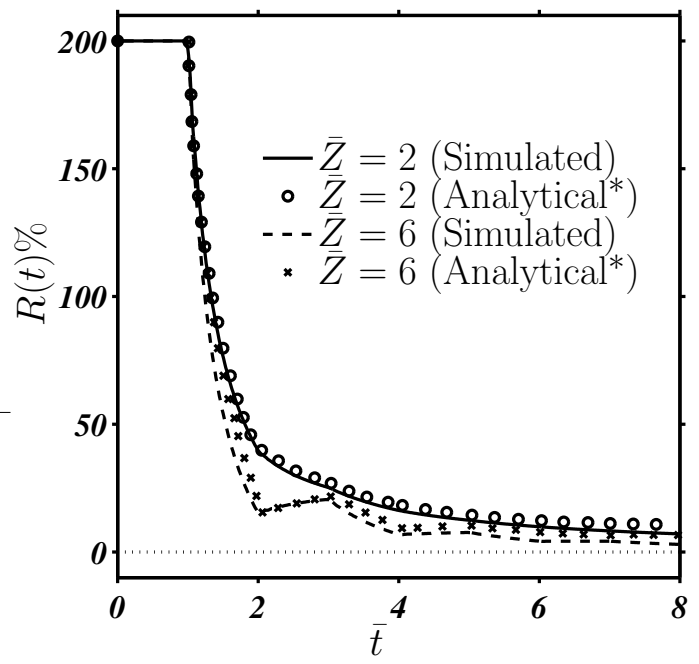

Figure 3.2: Comparison of simulated stress equilibration curves for a ceramic specimen being tested in a SHPB setup with analytical results.

tained against number of wave reflection $\bar{t}=t / t_{0}$. In the present study, all the time quantities are normalized with $t_{0}=L / c_{d}$ which represents the time required by the dilatational wave $\left(c_{d}\right)$ to travel the bulk specimen length $(L)$. Figure 3.2 compares the simulated stress equilibration curves with analytical results obtained by Ravichandran and Subhash (1994). It can be found that our results are in good agreement with the one-dimensional stress wave propagation through a long slender bar studied by these authors.

One of the prime issues associated with the testing of soft cellular structures in SHPB is that it is difficult to ensure stress equilibration in the specimen due to low impedance with respect to the bars and the attendant attenuation of stress wave as it travels through the specimen. Note that $R(t)$ defined in Equation (3.2) is a pointwise measure of stress equilibration at a particular time instant. It is a workable measure for ceramic specimens as the stress equilibration history for these materials in a SHPB set up shows a monotonic decrease after initial transients for all the cases of impedance mismatch (Figure 3.2). But for soft materials, due to possible deformation localization

\footnotetext{
* Ravichandran and Subhash (1994)
} 
(cell collapse), $R(t)$ can fluctuate even after a large number of wave reflections in the specimen.

\subsubsection{A typical stress equilibration study}

As an example, we present the stress equilibration history for a cellular specimen with $L=750 \mu m, D=1.5 L$, and $\bar{Z}=\bar{Z}_{I}=\bar{Z}_{T}=6$ in Figure 3.3 . The specimen is made

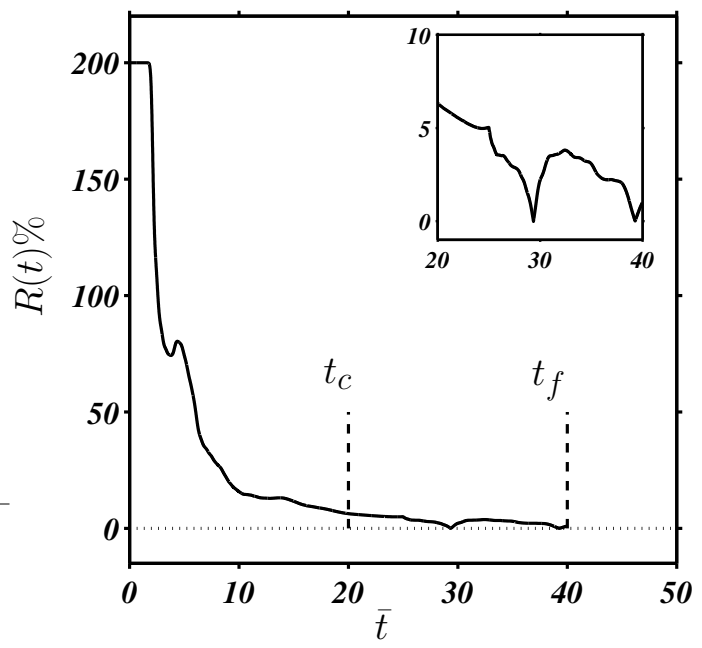

Figure 3.3: Typical stress equilibration curve $R(t)$ against normalized time $\left(\bar{t}=t / t_{0}\right)$ as obtained for a cellular material. The stress equilibration measure, as characterized by RMS value of the signal, is defined over the time period between $t_{c}$ and $t_{f}$. Inset shows the blow up of signal $R(t)$ over a time range of $t_{c}=20 t_{0}$ and $t_{f}=40 t_{0}$.

of polyethylene with $E=0.15 \mathrm{GPa}, \rho_{s}=910 \mathrm{kgm}^{-3}$ and $\nu=0.3$, and is subjected to an impact velocity $v=0.75 \mathrm{~m} / \mathrm{s}$. Notice that, it takes about 20 reflections for $R(t)$ to come down within $5 \%$ while only 8 reflections were sufficient for ceramic specimen (Figure 3.2). Moreover, even after 20 reflections, $R(t)$ shows significant oscillations (shown in inset of Figure 3.3) although overall trend is monotonically decreasing. To capture this general trend, a time averaged measure for stress equilibration is proposed in this paper. We introduce $R_{r m s}$, the root mean square value of $R(t)$ calculated over 
some prescribed time span $t_{c}$ to $t_{f}$ as

$$
R_{r m s}=\sqrt{\frac{1}{t_{f}-t_{c}} \int_{t_{c}}^{t_{f}} R(t)^{2} d t} .
$$

Calculation of $R_{r m s}$ is started at the time instant $t_{c}$ when $R(t)$ is within a certain bound chosen by the user (Figure 3.3), while $t_{f}$ is chosen in a way such that enough number of stress wave reflections have occurred to achieve the stress equilibration. When the RMS value of the stress difference becomes $R_{r m s} \leq 5 \%$, it is assumed that the specimen has reached stress equilibration. In order to find suitable values of $t_{c}$ and $t_{f}$, several simulations are performed for different cellular materials with a wide range of relative densities and loading range but with a fixed impedance mismatch $\bar{Z}_{I}=\bar{Z}_{T}=6$. It was found that a minimum of 20 wave reflections are required for the soft cellular structures under considerations to reach an acceptable state of dynamic equilibration. Furthermore, all simulations are stopped at 40 reflections which is sufficient to evaluate the RMS value of $R(t)$. Henceforth, in the present analysis we have adopted $t_{c}=20 t_{0}$ and $t_{f}=40 t_{0}$. Finally, it should be noted here that for highly localized deformations, $R(t)$ may show significant oscillations, its overall trend may not be decreasing, and stress equilibration can never be achieved.

\subsubsection{Intrinsic parameters of the model}

In the literature, it can be found that the mechanical properties of cellular materials

are represented in terms of their relative density (Gibson and Ashby, 1997). Relative density combines all the microstructural features (such as cell size, cell wall thickness etc.) with bulk material properties (material density). For a fixed value of material density $\rho_{s}$, a low relative density signifies a cellular structure containing large pores and/or thin struts, whereas higher relative density cellular materials contain smaller 
cells and/or higher strut thickness.

With a uniform strut thickness of $h_{s}$ for all the cells, relative density of a cellular structure can be defined as

$$
\bar{\rho}=\frac{\rho}{\rho_{s}}=\frac{\sum_{i=1}^{n_{s}} l_{i} \xi h_{s}^{2}}{L D h_{s}},
$$

where $\rho$ is the apparent density of cellular structure, $\rho_{s}$ is the bulk material density of the cellular specimen, $n_{s}$ is the number of struts, $l_{i}$ are the length of struts and the parameter $\xi$ is a dimensionless parameter depending upon the shape of the strut cross-section. Introducing aspect ratio $\phi=L / D$ and another dimensionless parameter $\bar{\kappa}=L / d_{a v}$ that represents average number of cells in the specimen length direction and substituting in Equation (3.7), $\bar{\rho}$ can be recast as

$$
\bar{\rho}=\frac{\sum_{i=1}^{n_{s}} l_{i} \xi}{d_{a v}} \frac{h_{s}}{d_{a v}} \frac{\phi}{\bar{\kappa}^{2}} .
$$

From this equation, relative density $\bar{\rho}$ can be expressed as a function of normalized parameters $\bar{\eta}, \bar{d}_{a v}, \phi$ and $\bar{\kappa}$ as

$$
\bar{\rho}=f\left(\bar{\eta}, \bar{d}_{a v}, \phi, \bar{\kappa}\right)
$$

In the above equation, $\bar{\eta}$ is defined as $\bar{\eta}=\sum l_{i} / N_{c} d_{a v}$, with $N_{c}$ representing total number of cells in the specimen while $\bar{d}_{a v}=d_{a v} / h_{s}$ is the average cell size normalized with strut thickness $h_{s}$. It should be noted that $\bar{\eta}$ represents the ratio of one half perimeter to mean diameter of each cell (in a perfectly regular array) in the domain and hence depends on the shape of the cells. The value of $\bar{\eta}$ remains constant even in the presence of moderate randomization in cell shape and size, as long as the average cell shape is maintained. For example, for a perfect honeycomb microstructure, $\bar{\eta}$ is 1.66. We have generated a number of cellular microstructures with different $d_{a v}$ but 
a constant standard deviation of $0.02 d_{a v}$ for a domain with different values of $\phi$ while keeping strut thickness $h_{s}$ constant. Resulting variation of $\bar{\eta}$ with $\bar{\rho}$ has been plotted

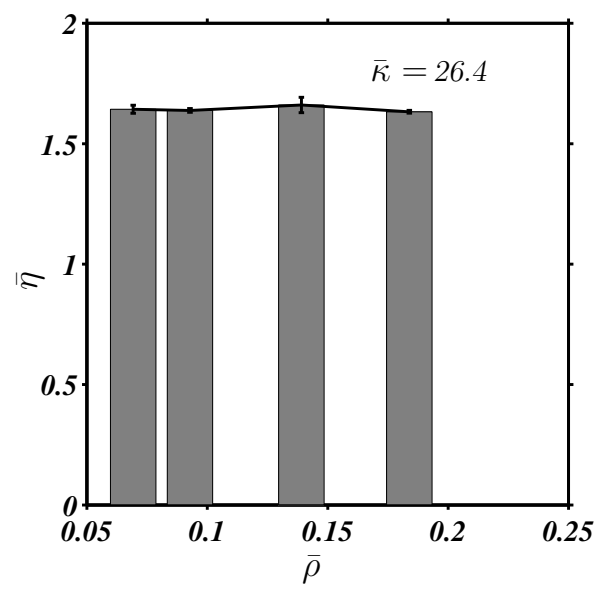

Figure 3.4: Variation of $\bar{\eta}$ with respect to relative density of the specimen with $\bar{\kappa}=26.4$. Error bars represent the variation of $\bar{\eta}$ with respect to aspect ratio $\phi$ for each instance of the relative density $\bar{\rho}$.

in Figure 3.4. It can be observed that, for different relative densities (corresponding to different $\bar{d}_{a v}$ and a fixed $\left.\bar{\kappa}\right), \bar{\eta}$ remains almost constant. Also, the effect of aspect ratio $\phi$ on $\bar{\eta}$, shown as error bars in Figure 3.4, is negligible. So, it can be inferred that, even in the presence of a small randomization in cell size, parameter $\bar{\eta}$ does not affect the relative density of the cellular specimen appreciably. But a change in shape of cells or a large variation in cell size in the microstructure will affect $\bar{\eta}$ and will be reflected in the relative density and subsequent dynamic behavior of the material. For the current study, we have used only nearly hexagonal cell shapes with $\bar{\eta}$ close to 1.66 for all the cases. So we will not consider the effect of $\bar{\eta}$ in subsequent simulations. In the following, the effect of two major parameters, the number of cells in specimen length $\bar{\kappa}$ and the normalized cell size $\bar{d}_{a v}$, which exhibit a strong effect on the relative density and hence on the overall dynamic behavior of cellular materials, will be characterized in detail for different extrinsic parameters such as loading rate and impedance mismatch. We will also examine the effect of specimen aspect ratio $\phi$ on the stress equilibration process in this class of materials. 


\subsection{Result and discussions}

Using the numerical scheme described in Chapter 2, dynamic stress equilibration process in a split Hopkinson pressure bar setup is studied for a wide range of cellular structures. A slightly disordered honeycomb microstructure (Figure 2.9(a)), which is essentially two dimensional, has been used for all the simulations outlined in this paper. Cell size distribution of these microstructures can be described by the mean cell diameter $d_{a v}$ and a standard deviation $S_{d}$, as discussed in Chapter 2. It is assumed that the distribution of the cell size arises from minor processing induced irregularities in the microstructure, and accordingly, a small $S_{d}=0.02 d_{a v}$ is used to characterize all the specimens in the current study. Specimen cell walls (struts) are assumed to be square in cross-section leading to $\xi=1$ in Equation (3.7). Polyethylene, a polymer whose material properties are given by $E=0.15 \mathrm{GPa}, \rho_{s}=910 \mathrm{kgm}^{-3}$ and $\nu=0.3$, is chosen as the constituent phase of the cellular specimen (Gibson and Ashby, 1997) used in this study. It is assumed that the material remains elastic throughout the simulation process though it can exhibit considerable geometrically nonlinear response. As the incident stress signal is proportional to striker velocity in a SHPB setup, a

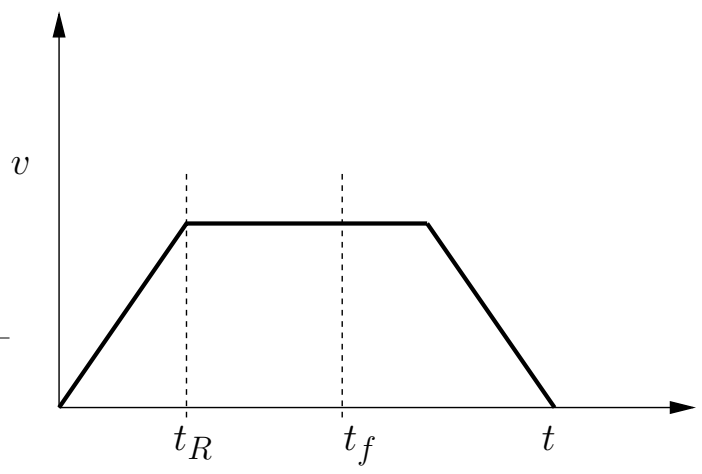

Figure 3.5: Trapezoidal loading pulse with a velocity $v$ and rise time $t_{R}$. Final time $t_{f}$ corresponds to a time instant up to which the stress equilibration measure $R_{r m s}$ is computed.

velocity pulse is considered here instead of stress input. A typical velocity pulse in SHPB is described in Figure 3.5 where $v$ is the magnitude of the applied velocity 
and $t_{R}$ is the rise time of the pulse, both of which are systematically varied in our simulations. Applied velocity is normalized with dilatational wave speed $c_{d}$ of the cellular bulk material (polyethylene) as $\bar{v}=v / c_{d}$. Normalized rise time is expressed as $\bar{t}_{R}=t_{R} / t_{0}$ while final time $\bar{t}_{f}=t_{f} / t_{0}$ corresponds to the time instant at which the calculation of $R_{r m s}$ is terminated, $t_{0}$ being the travel time of the dilatational wave through the bulk specimen length. Incident and transmission bars are made of the same material in a typical SHPB set up (Subhash et al., 2006a). Accordingly, in the present analysis, impedance mismatch ratios of the incident bar as well as the transmission bar with the specimen are kept the same, and are denoted as $\bar{Z}_{I}$ and $\bar{Z}_{T}$, respectively. In the ensuing discussion, we detail the effects of various intrinsic parameters such as average cell size, specimen dimension (in terms of number of cells in specimen length) and extrinsic parameters such as, loading rate and impedance mismatch systematically. These effects are quantified through various nondimensional parameters, $\bar{d}_{a v}, \phi$, and $\bar{\kappa}$, as introduced in Section 3.3 .

\subsubsection{Effect of cell size $d_{a v}$ and number of cells $\bar{\kappa}$ in the spec- imen length}

In this section, we examine the effect of number of cells in the specimen length $\bar{\kappa}$ for a range cell size $d_{a v}$ varied systematically. In order to do that, specimens containing different number of cells in the specimen length $(\bar{\kappa}=3.3$ to 26.4) for a range of cell sizes varying from $d_{a v}=28.4$ to $75.4 \mu \mathrm{m}$ are generated keeping the strut thickness $h_{s}=2.5 \mu \mathrm{m}$ and specimen aspect ratio $\phi=1 / 1.5$ for all the cases. Resulting relative density variation is given in Figure 3.6 for different normalized average cell size $\bar{d}_{a v}$. It can be observed that relative density $\bar{\rho}$ decreases nonlinearly as the normalized cell

size $\bar{d}_{a v}$ increases for a fixed strut thickness and aspect ratio. Overlapping curves for different $\bar{\kappa}$ signify that the relative density does not depend on number of cells in the 


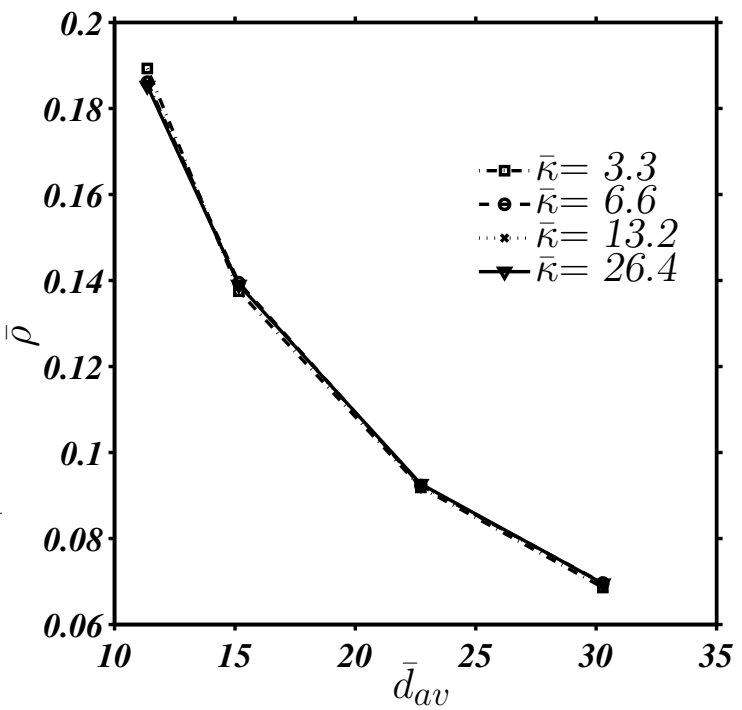

Figure 3.6: Variation of relative density $\bar{\rho}$ with normalized average cell size $\bar{d}_{a v}$ and number of cells in the specimen length $\bar{\kappa}$ for a fixed strut thickness $h_{s}=2.5 \mu \mathrm{m}$ and aspect ratio $\phi=1 / 1.5$.

length directions for a given cell size $\bar{d}_{a v}$ with fixed aspect ratio $\phi$ and strut thickness $h_{s}$. For all the specimens as mentioned above, a trapezoidal loading pulse with an applied maximum loading velocity $\bar{v}=0.01$ (resulting in a strain rate $\dot{\varepsilon}=5 \times 10^{3} \mathrm{~s}^{-1}$ ) and a normalized rise time $\bar{t}_{R}=25$ is employed. Such a rise time offers enough wave reflection within the specimen thus ensuring the stress equilibrium of specimen. The effect of rise time on stress equilibration will be demonstrated in the subsequent discussions. Simulations are run up to 40 wave reflections in the specimen resulting in a normalized final time $\bar{t}_{f}=40$. Specimens are subjected to equal impedance ratios on both the ends with $\bar{Z}=\bar{Z}_{I}=\bar{Z}_{T}=6$.

Figure 3.7 depicts the variation of stress equilibration measure $R_{r m s}$ with normalized cell size $\bar{d}_{a v}$ and number of cells in the specimen length $\bar{\kappa}$. Observe from this figure that $R_{r m s}$ decreases rapidly with decreasing $\bar{d}_{a v}$ for a fixed value of $\bar{\kappa}$, and consequently, stress equilibration is better achieved at a final given time $\bar{t}_{f}$. This trend can be observed for all values of $\bar{\kappa}$. As it will be seen later, localized deformations that cause oscillations in the stress equilibration process (Figure 3.10(b)) are delayed or 


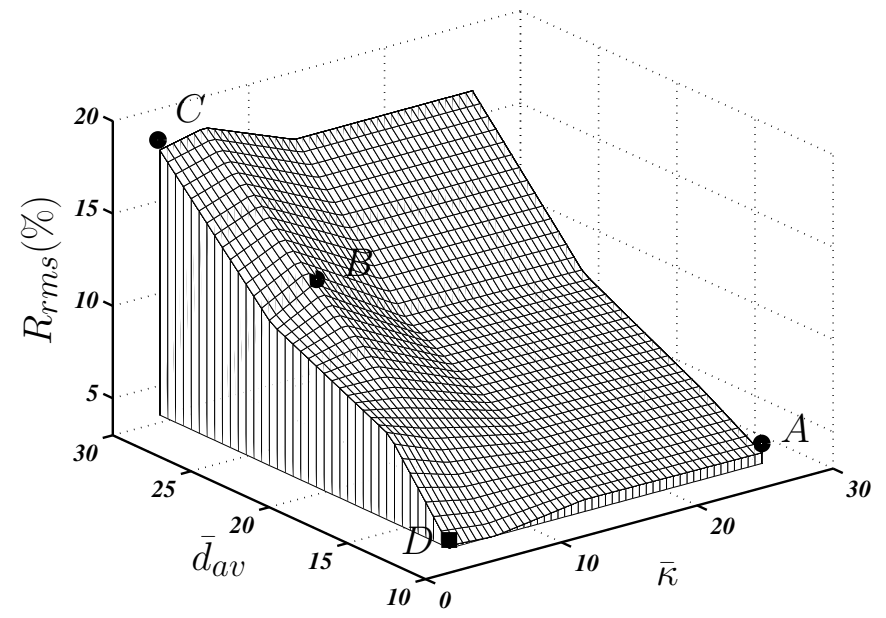

Figure 3.7: Variation of $R_{r m s}$ with number of cells along the specimen length $\bar{\kappa}$ for a range of normalized cell size $\bar{d}_{a v}$. Strut thickness $h_{s}$ is held constant of $2.5 \mu \mathrm{m}$ while aspect ratio $\phi=1 / 1.5$ for all the simulations.

altogether eliminated at low $\bar{d}_{a v}$ (high relative density) resulting in a low value of $R_{r m s}$. Note from Figure 3.7 that achieving a low value of $R_{r m s}$ is difficult at higher $\bar{d}_{a v}$ even if there are a large number of cells in the length direction. On the other hand, stress equilibration can be achieved with just a few cells $(\bar{\kappa}=3.3)$ in the length direction (marked by square $D$ in Figure 3.7) if normalized cell size is sufficiently low (higher relative density). But too few cells may not give a representative (converged) stress-strain response, as will be shown later in this section, and should be avoided.

The observations described above are based on normalized average cell size $\bar{d}_{a v}$ and number of cells in specimen length $\bar{\kappa}$ with an aspect ratio of $1 / 1.5$. In order to examine the effect of specimen geometry on the stress equilibration process, two sections (parallel to $\bar{d}_{a v}$ and $\bar{\kappa}$ axes respectively) corresponding to $\bar{d}_{a v}=15.1$ and $\bar{\kappa}=26.4$ are taken from Figure 3.7 and the aspect ratio $\phi$ is varied from $1 / 0.5$ to $1 / 1.75$. Error bars in Figure 3.8(a) show the deviation of $R_{r m s}$ for the above range of aspect ratios for selected values of $\bar{\kappa}$ with a constant normalized average cell size $\left(\bar{d}_{a v}=15.1\right)$. Similarly in Figure 3.8(b), the effect of aspect ratio, again represented by error bars, is presented for different $\bar{d}_{a v}$ but for a fixed number of cells along the specimen length 
$(\bar{\kappa}=26.4)$. Observe from these figures that $R_{r m s}$ remains largely unaffected by the

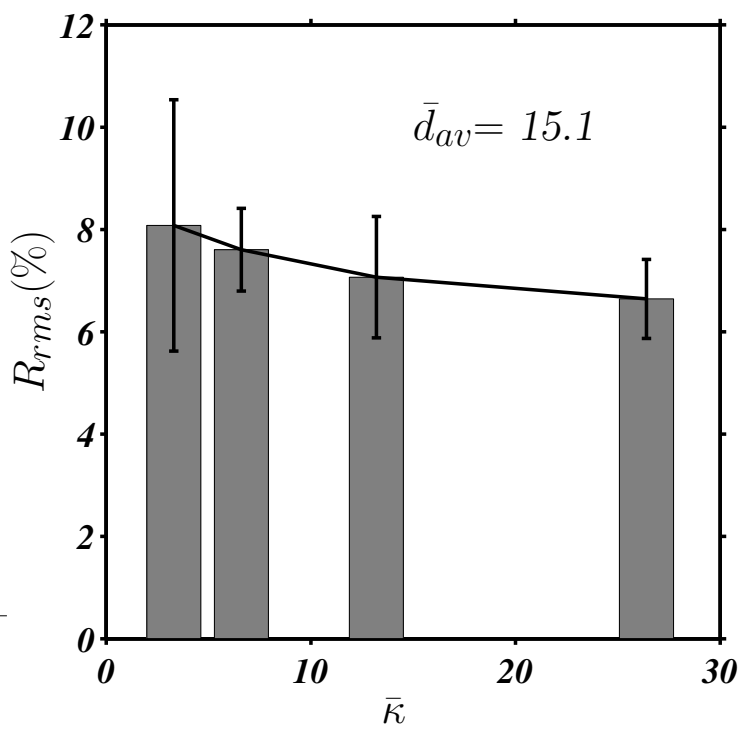

(a)

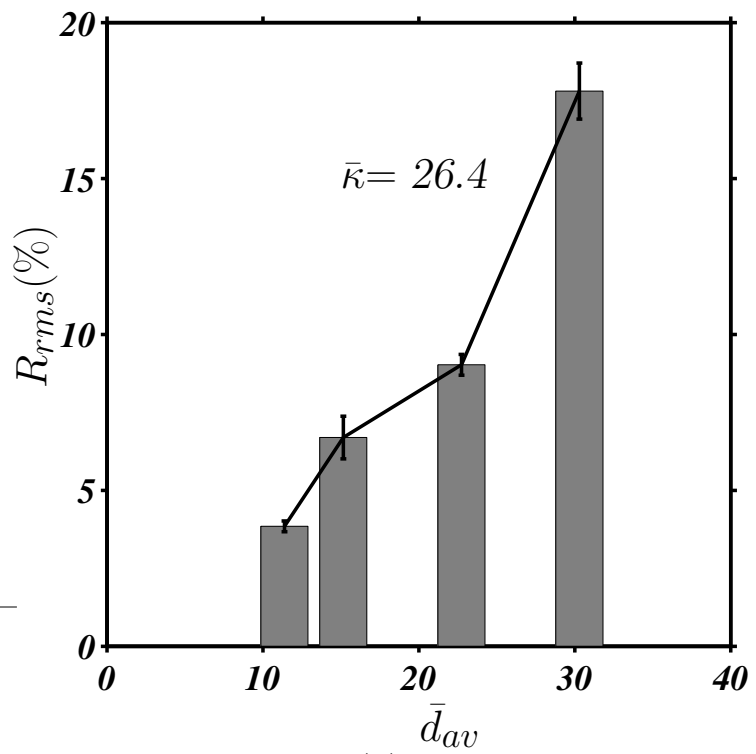

(b)

Figure 3.8: $\quad$ Effect of aspect ratio $\phi$, represented by error bars, on $R_{r m s}$ (a) for a constant normalized average cell size $\left(\bar{d}_{a v}=15.1\right)$ but varying $\bar{\kappa}$, and (b) for a fixed number of cells $(\bar{\kappa}=26.4)$ but different $\bar{d}_{a v}$.

variation of specimen aspect ratio $\phi$ except for the very low value of $\bar{\kappa}$, where the variations are the maximum. So it can be concluded that, at least for the range of 
$\phi$ chosen in this study, stress equilibration process is not influenced by the specimen length to width ratio when sufficient number of cells are present in the length direction. As expected, stress equilibration is strongly affected by the average cell size in the specimen (Figure 3.8(b)). For a fixed number of cells in the length direction $(\bar{\kappa}=26.4)$, larger the cell size, more difficult it is for stress equilibration to occur (i.e. higher $R_{r m s}$ value).

Average number of cells present in the specimen length, represented by $\bar{\kappa}$, does not affect the stress equilibration process significantly as found in Figures 3.7 and 3.8(a). But the stress-strain response of the cellular structure, as extracted from the SHPB test, may depend on the average number of cells in the specimen length. Even though a specimen with a low value of $\bar{\kappa}$ provides an equilibrium stress state for a given loading scenario, (Figures 3.7 and 3.8(a)), number of cells in the specimen length may not be statistically significant to provide actual cellular structure constitutive response. To elucidate this point, $\bar{\kappa}$ is varied from 3.3 to 26.4 for a specimen with a fixed cell size $\bar{d}_{a v}=15.1(\bar{\rho}=0.139)$. From Figure 3.9, it can be found that for only three layers of cells in the length direction $(\bar{\kappa}=3.3)$, the constitutive response is reminiscent of the single cell stress-strain response (?) rather than the average cellular material response sought. As $\bar{\kappa}$ increases, the stress-strain response converges towards the actual constitutive response of a soft cellular specimen. Thus, it can be concluded that although the number of cells (in specimen length) required for stress equilibration could be small for higher relative density cellular materials, for effective stress-strain response, at least 15 layers of cells $(\bar{\kappa}=13.2)$ are required. It should be mentioned that the least value of $\bar{\kappa}$ for other choices of material properties, cell geometries and loading conditions can be different from this value. But, just a few cells in length direction will never sufficiently capture the dynamic response of the material.

As discussed in Section 3, to characterize the stress equilibration, we have introduced an average value of the stress equilibration measure, $R_{r m s}$, over a finite interval of 


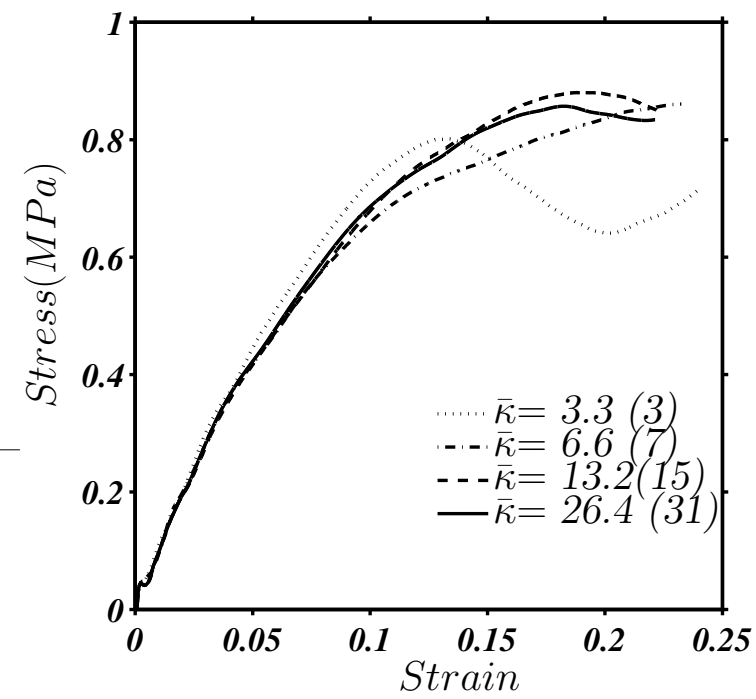

Figure 3.9: Stress-strain response of cellular specimens with a constant cell size $\left(\bar{d}_{a v}=15.1\right)$ but different number of normalized cells in the specimen length $(\bar{\kappa})$. Numbers in parentheses indicate actual number of layers of cells in the specimen length direction.

time. Motivation behind this averaging process was to extract equilibration information in the presence of oscillations in the stress equilibration history (Figure 3.3). Recall that it requires a large number of wave reflections at the interfaces to reach stress equilibration in the entire cellular specimen. Ravichandran and Subhash (1994) used an $R(t)$ value of $5 \%$ as an acceptable measure for stress equilibration. This resulted in eight wave reflections within the ceramic specimen after which the stress-strain response was considered valid. For cellular structures, this value may be unrealistic to achieve due to reasons stated earlier. In the ensuing discussion, we study the actual evolution of $R(t)$ for various cellular structures and its relation to the extracted constitutive response. To accomplish this task, three points $(A, B$ and $C)$ from $\bar{d}_{a v^{-}} \bar{\kappa}$ space in Figure 3.7 are selected such that two points $(A$ and $C$ ) represent the extreme situations (high $\bar{d}_{a v}$ and low $\bar{\kappa}$, and vice versa) and the third point $(B)$ lies in between them. These three cases correspond to $\bar{d}_{a v}=11.3,22.7$ and 30.3 with $\bar{\kappa}=26.4,6.6$ and 3.3, respectively, and $R_{r m s}$ values at these points are given in the Table 3.1.

Stress-strain responses corresponding to these three points are shown in Figure $3.10(\mathrm{a})$ 


\begin{tabular}{llllll}
\hline \hline & Points & $\bar{d}_{a v}$ & $\bar{\kappa}$ & $\bar{\rho}$ & $R_{r m s}(\%)$ \\
\hline Case I & $A$ & 11.3 & 26.4 & 0.189 & 4.1 \\
Case II & $B$ & 22.7 & 6.6 & 0.09 & 12.5 \\
Case III & $C$ & 30.3 & 3.3 & 0.07 & 18.2 \\
\hline
\end{tabular}

Table 3.1: $R_{r m s}$ values for chosen combinations of $\bar{d}_{a v}$ and $\bar{\kappa}$.

and corresponding stress equilibrium curves are given in Figure 3.10(b) as a function of number of wave reflections $\bar{t}=t / t_{0}$ in the specimen, where $t$ is the elapsed time. Points $\bar{t}_{i}$ for $i=1$ to 4 represent $\bar{t}=10,20,30$ and 40 , respectively, in these figures. It can be found from the stress equilibration curves that the specimen with cell size $\bar{d}_{a v}=11.3$ $(\bar{\rho}=0.189)$ maintains a lower stress difference $(R(t)<5 \%)$, and hence better stress equilibration between two ends when $\bar{t}>20$. Number of cells in the length direction also being high $(\bar{\kappa}=26.4)$, the stress-strain response presented in Figure $3.10(\mathrm{a})$ for this case can be taken as the representative dynamic response of the material. Note that, reasonable stress equilibration has been achieved within 25 reflections for this case. For Case II (point $B$ ) with an intermediate $\bar{d}_{a v}$ of 22.7 and $\bar{\kappa}=6.6$ (shown by dash-dot line in Figure 3.10(b)), there are severe oscillations throughout $R(t)$ history, but they are limited to less than $R(t)=10 \%$ for $\bar{t}>25$. This fact is reflected by an $R_{r m s}$ value of $12.5 \%$ for this case (Table 3.1). With the acceptable limit of $R_{r m s}$ fixed at, say $5 \%$, the stress equilibration could not be achieved and maintained throughout the test process for this case. However, if this condition is relaxed to, say $10 \%$, the equilibration condition can be satisfied for $\bar{t}>25$ in an average sense. However for Case III (point $C$ ), the low relative density coupled with very few number of cells in the specimen length direction fails to establish stress equilibration at all except for small intervals. This fact is clearly reflected in Figure 3.10(b). Corresponding stressstrain curve (Figure 3.10(a), dotted line) is therefore not a valid constitutive response for this case. The above discussion clearly highlights the dependence of stress-strain response on the number of cells in the specimen length direction. With increased number of cells, it is easier to establish stress equilibration during the test and obtain a valid stress-strain response that is representative of the cellular structures. 


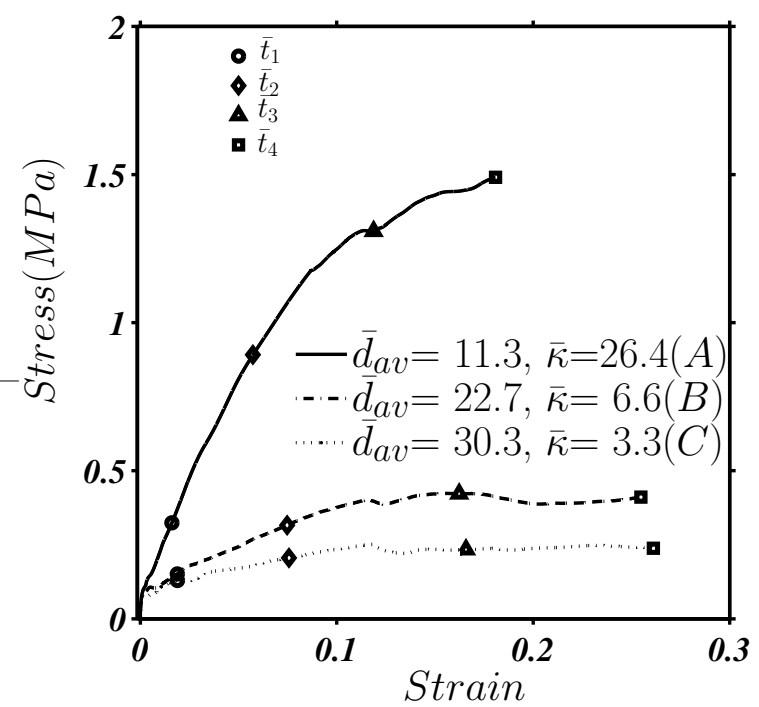

(a)

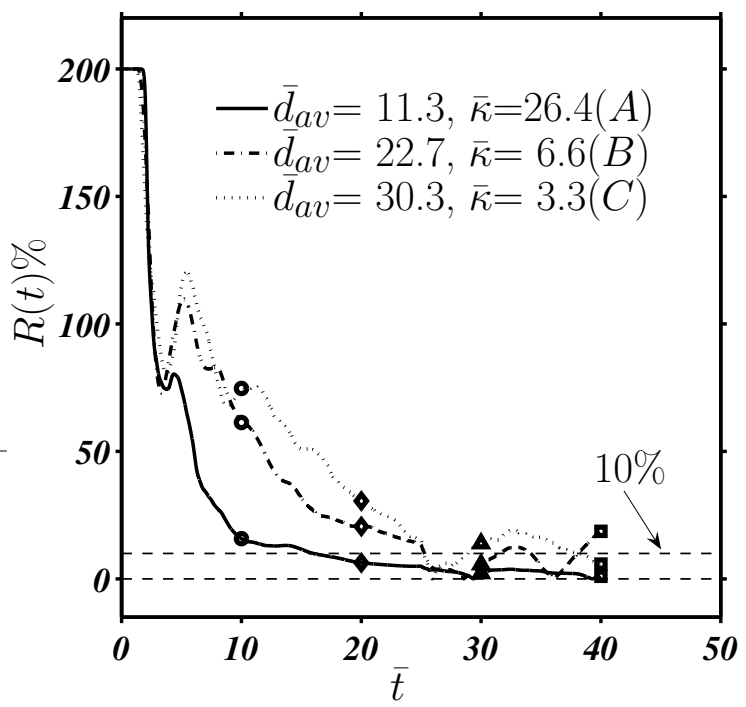

(b)

Figure 3.10: (a) Stress-strain curves for cellular specimen for the three cases designated in Table 3.1, and (b) corresponding stress equilibrium history.

To identify the deformation features of the above mentioned cellular structures, snapshots of deformed structure at selected intervals $\bar{t}_{i}, i=1,4$, are shown in Figure 3.11. For clarity, the degree of deformation in each cell is defined by $A_{c}^{n} / A_{c}^{o}$, where $A_{c}^{o}$ and $A_{c}^{n}$ are the cell areas at $t=0$ and $t=t_{n}$, respectively, and is represented by a grey 


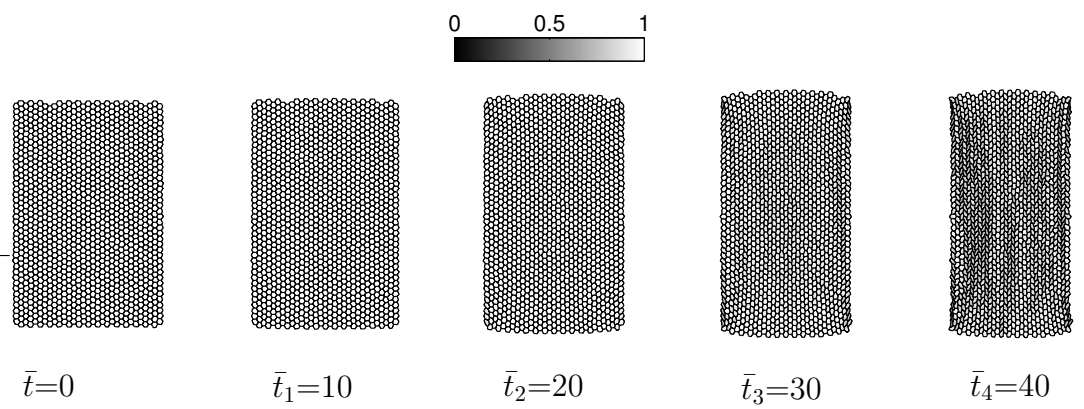

(a) $\bar{d}_{a v}=11.3$ and $\bar{\kappa}=26.4$

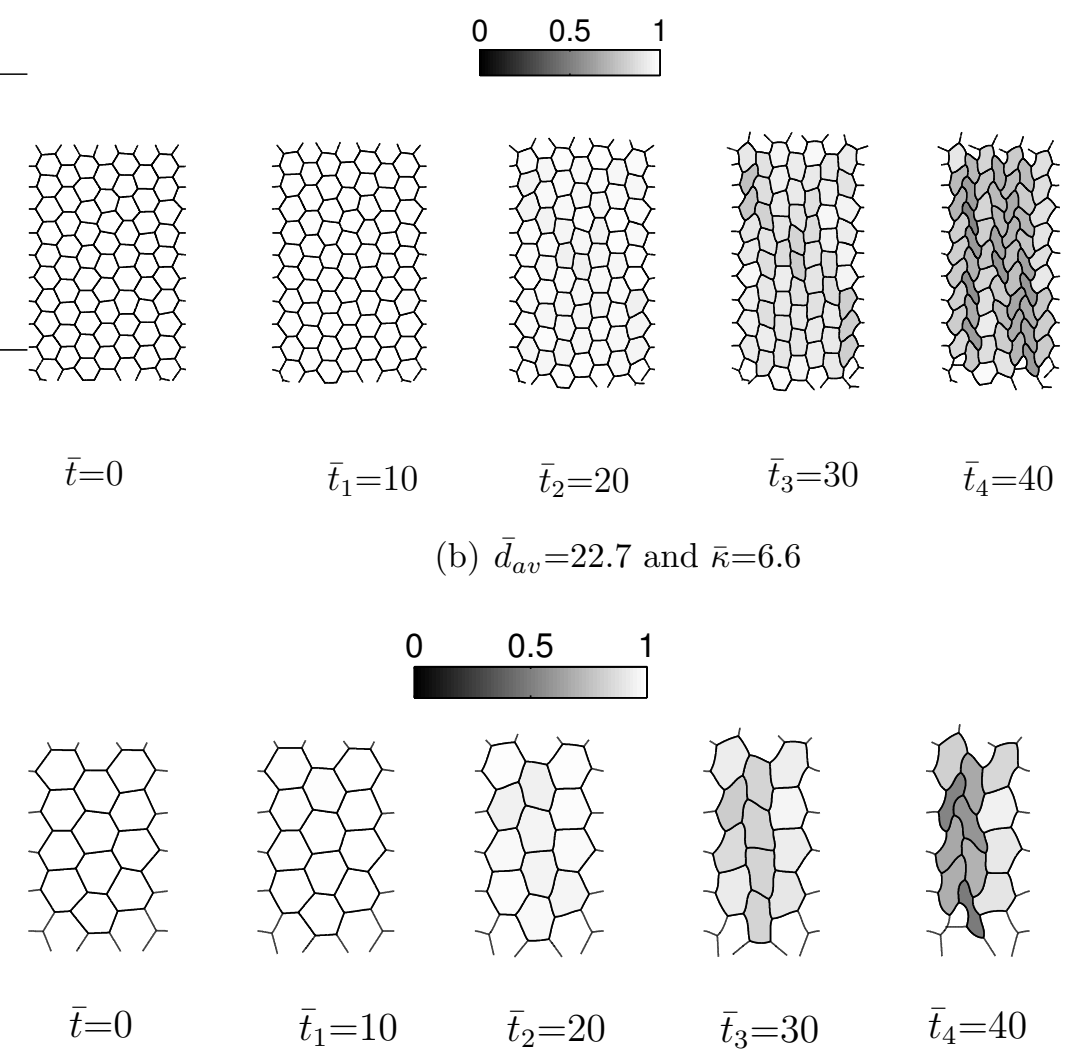

(c) $\bar{d}_{a v}=30.3$ and $\bar{\kappa}=3.3$

Figure 3.11: Snapshots of deformed specimens at selected time intervals for the three cases $(A, B$ and $C$ ) designated in Table 3.1. Degree of deformation in each cell is represented by a grey scale varying from 0 (no deformation) to 1 (full collapse).

scale with $A_{c}^{n} / A_{c}^{o}=1$ representing the undeformed cell (colored white) and $A_{c}^{n} / A_{c}^{o}=0$ indicating a fully collapsed cell (colored dark). However, we did not assign a grey scale for incomplete cells at the boundaries, and consequently, they show in white 
in the figure. It can be seen from the Figure 3.11(a) that for the specimen with the smallest cell size (Case I), deformation gradients are not very localized; they are rather spread throughout the domain. For Case II (Figure 3.11(b)), a deformation gradient over the domain can be observed which could be the result of lower bending stiffness due to larger cell size (lower relative density). In Figure 3.11(c), specimen with largest cell size (Case III) shows a higher deformation gradient in the domain compared to the other cases. Such localized deformation features cause oscillations in the stress equilibration curves (Figure 3.10(b)) and result in unequal stress values at the two ends of the specimen. Stress-strain response extracted in these situations are not representative for the actual cellular materials; they rather contain the effects of these localized regions.

\subsubsection{Effect of loading rate}

So far in the discussion, the effect of cell size and number of cells along the specimen length are analyzed. It is well known that applied loading rate also plays a significant role in dynamic response of cellular materials. As mentioned earlier, understanding of stress equilibration in the specimen is a necessary prerequisite for correct evaluation of material response by SHPB using proper data reduction method. Towards this end, we will first focus on the effect of loading rate on stress equilibration. As discussed in Section 3.4, loading rate is characterized by two parameters: rise time of the pulse $t_{R}$ and maximum applied velocity $v$ (Figure 3.5) at the incident bar-specimen interface. Initially, maximum normalized applied loading velocity $\bar{v}$ is varied while keeping the normalized pulse rise time $\bar{t}_{R}$ and average number of cells in the specimen length $\bar{\kappa}$ constant. Accordingly, we adopted $\bar{\kappa}=13.2$ while cell size $d_{a v}$ is varied from 28.2 to $75.7 \mu \mathrm{m}$ with a strut thickness $h_{s}=2.5 \mu \mathrm{m}$ and an aspect ratio $\phi=1 / 1.5$. This set of parameters resulted in specimens with varying relative density $\bar{\rho}$ from 0.19 to 0.07 , 


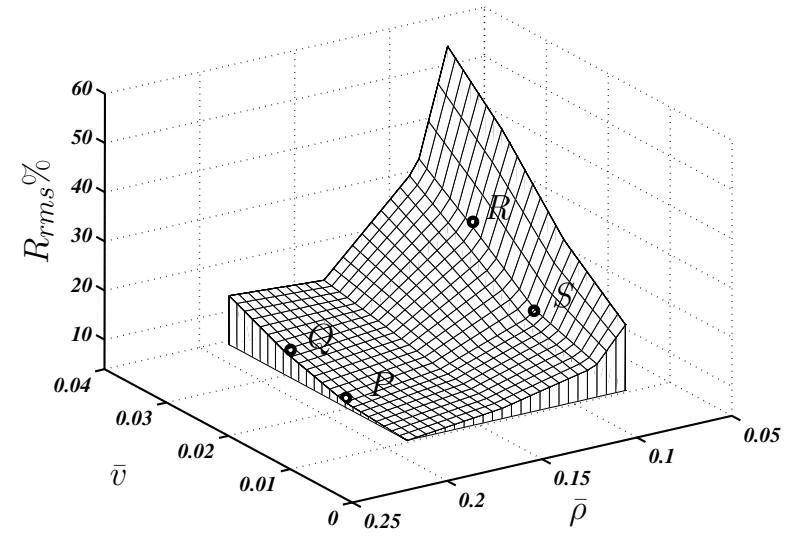

Figure 3.12: RMS values of the stress equilibration measure $\left(R_{r m s}\right)$ as a function of relative density $\bar{\rho}$ and maximum applied velocity $\bar{v}$ with a constant rise time $\left(\bar{t}_{R}=25\right)$.

respectively (Figure 3.6). All the specimens are subjected to an equal impedance mismatch ratio $\bar{Z}=\bar{Z}_{I}=\bar{Z}_{T}=6$ at the ends. The variation of $R_{r m s}$ is calculated as a function of the above range of relative density $\bar{\rho}$ and the maximum applied velocity $\bar{v}$ for a trapezoidal loading pulse with a rise time of $\bar{t}_{R}=25$. Figure 3.12 shows that higher relative density and lower amplitude of applied velocity leads to a quick establishment of the stress equilibration (i.e., lower $R_{r m s}$ ). On the other hand, stress equilibration is most difficult to achieve when relative density is low and magnitude of the applied velocity is simultaneously high.

In order to characterize the influence of loading rate on the stress-strain response and stress equilibration in cellular specimens, four points $(P, Q, R$ and $S)$ from the $\bar{v}-\bar{\rho}$ space in Figure 3.12 are chosen.

\begin{tabular}{llll}
\hline \hline Points & $\bar{\rho}$ & $\bar{v}$ & $R_{r m s}(\%)$ \\
\hline$P$ & 0.19 & 0.02 & 5.5 \\
$Q$ & 0.19 & 0.03 & 9.2 \\
$S$ & 0.09 & 0.02 & 15.5 \\
$R$ & 0.09 & 0.03 & 24.9 \\
\hline
\end{tabular}

Table 3.2: $R_{r m s}$ values for different $\bar{v}$ and $\bar{\rho}$ as chosen from Figure 3.12 . 
These cases correspond to a low density specimen $(\bar{\rho}=0.09)$ as well as a higher density one $(\bar{\rho}=0.19)$, subjected to two different applied velocities, $\bar{v}=0.02$ and 0.03 , respectively and their $R_{r m s}$ values are given in Table 3.2. Figure 3.13 shows the stress-strain responses as well as the state of dynamic stress equilibration histories for these four cases. It can be seen that, even for a small increase in loading velocity, the specimen

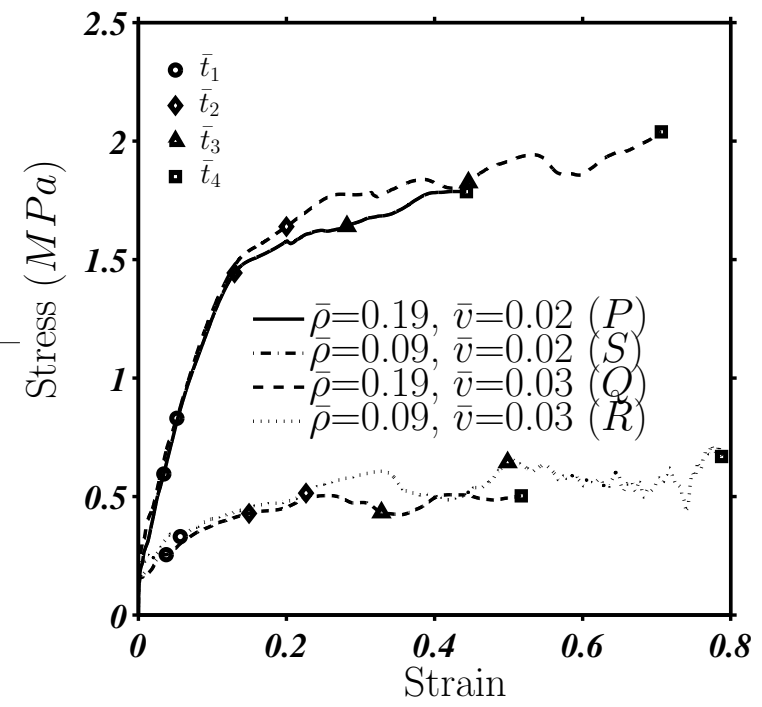

(a)

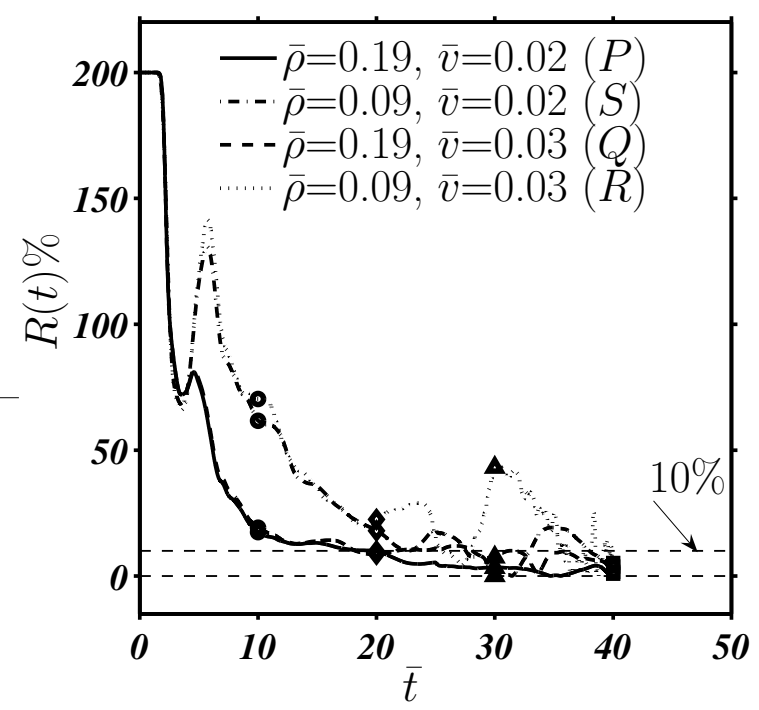

(b)

Figure 3.13: (a) Stress-strain response, and (b) stress equilibration curves for the four cases chosen from Figure 3.12 . 
with a higher relative density $(\bar{\rho}=0.19$, point $Q)$ starts to deviate from the equilibration (e.g. point $P$ ) and gives rise to an oscillatory stress-strain response (dashed line in Figure 3.13(a)). An equal increase in loading velocity for a lower relative density specimen $(\bar{\rho}=0.09$, point $R)$ affects the stress equilibration more severely (dotted line in Figure $3.13(\mathrm{~b})$ ) with an $R_{r m s}$ of $24.9 \%$, and leads to an invalid stress-strain response for the specimen.

Loading rate can also be controlled by changing the rise time of the pulse. Influence of pulse shapes with different pulse rise times $t_{R}$ and constant magnitude of applied velocity is also investigated. Similar to previous analysis, specimens with $\bar{\kappa}=13.2$ and varying cell size $d_{a v}$ (from 28.2 to $75.7 \mu \mathrm{m}$ ) with strut thickness $h_{s}=2.5 \mu \mathrm{m}$ and aspect ratio $\phi=1 / 1.5$ are subjected to an applied loading velocity of $\bar{v}=0.01$ with different normalized pulse rise times $\bar{t}_{R}$. Figure 3.14 shows the contour plots of RMS values of stress equilibration measure $R_{r m s}$ for a range of relative densities (from 0.19 to 0.07 ) and pulse rise times as mentioned earlier. It can be seen that for selected range, the

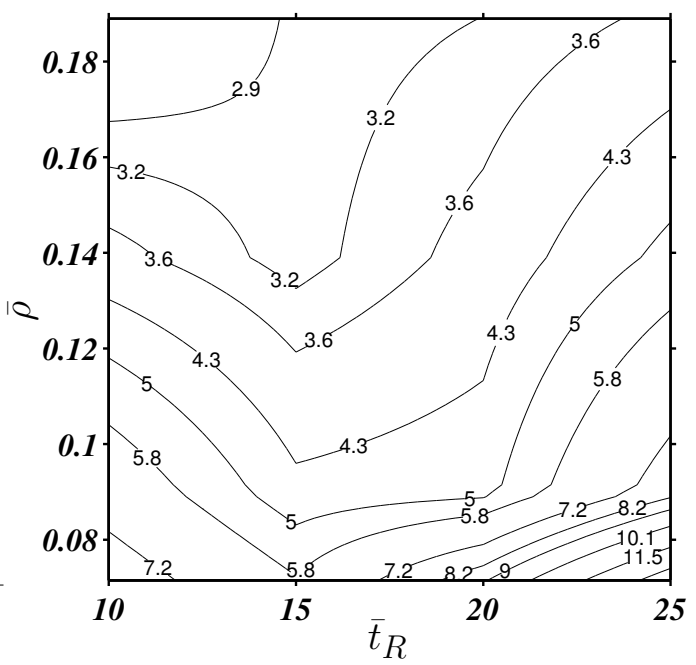

Figure 3.14: Contour plot of $R_{r m s}$ for different relative density materials with $\bar{\kappa}=13.2$, fixed applied velocity $\bar{v}=0.01$, and $\bar{Z}=\bar{Z}_{I}=\bar{Z}_{T}=6$ subjected to loading pulses with different rise times $\bar{t}_{R}$. All values of $R_{r m s}$ shown are in $(\%)$.

pulse rise time $\bar{t}_{R}$ has a less pronounced effect on the stress equilibration as $R_{r m s}$ 
varies only within a small range of 2 to $12 \%$. As expected, for higher relative density cellular specimens $(\bar{\rho}=0.19)$, the $R_{r m s}$ value is low in the selected range of $\bar{t}_{R}$ indicating that stress equilibration is easily reached for the parameters considered. For low relative density ( $\bar{\rho}=0.07)$ specimen, $R_{r m s}$ is marginally higher $(7-12 \%)$ indicating slightly increased difficulty to achieve the stress equilibration.

\subsubsection{Effect of end-impedance mismatch ratio}

Impedance mismatch ratio at both the interfaces of specimen and bars also plays a crucial role in stress equilibration and consequently the extraction of dynamic behavior of the specimen material. We have selected a wide range of impedance mismatch ratios in our simulations to study this effect. As mentioned earlier, these ratios at the incident face $\left(\bar{Z}_{I}\right)$ as well as the transmission face $\left(\bar{Z}_{T}\right)$ are kept at same value for all the simulations described, i.e. $\bar{Z}=\bar{Z}_{I}=\bar{Z}_{T}$. Similar to the previous discussion, specimens with $\bar{\kappa}=13.2$ and a range of cell size $d_{a v}$ from 28.2 to $75.7 \mu \mathrm{m}$ with strut thickness $h_{s}=2.5 \mu \mathrm{m}$ and aspect ratio $\phi=1 / 1.5$ are considered in this study. The specimens are subjected to a fixed loading pulse with a maximum applied velocity $\bar{v}=0.01$

and a pulse rise time $\bar{t}_{R}=25$. The contours in Figure 3.15 represent $R_{r m s}$ values of the stress equilibration measure for a range of relative densities from 0.19 to 0.07 , and impedance mismatch ratios $\bar{Z}$ from 1 to 8 . It can be seen that for a specimen with a fixed relative density, $R_{r m s}$ value decreases with increasing impedance mismatch ratios. For higher relative density specimens, even lower values of impedance mismatch ratios are sufficient to achieve satisfactory stress equilibration $(\approx 5 \%$ in our case), whereas, a low relative density specimen requires a higher impedance mismatch at the interfaces. A higher value of impedance mismatch causes a substantial portion of the stress pulse to be reflected from the interfaces, thus assisting to achieve stress 


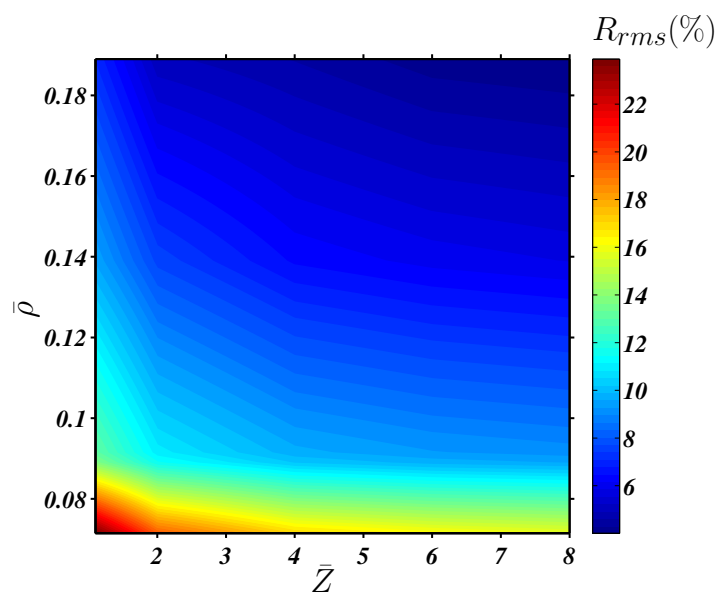

Figure 3.15: Contour plot of $R_{r m s}$ for different relative density materials subjected to different end impedance mismatch ratios $\bar{Z}=\bar{Z}_{I}=\bar{Z}_{T}$ with $\bar{\kappa}=13.2$, fixed applied loading $\left(\bar{v}=0.01\right.$ and $\left.\bar{t}_{R}=25\right)$. All values of $R_{r m s}$ shown are in $(\%)$.

equilibration faster. Although the higher value of impedance expedites the stress equilibration process, it may cause the transmitted signal to become weak resulting in difficulty in measurement. A higher $\bar{v}$ cannot always be used to alleviate this problem as this may cause localized deformations and associated lack of stress equilibration in the specimen. Thus, one has to strike a balance between the stress equilibration time and the amplitude of the transmitted signal in the SHPB experiment for a given set of bar material impedance mismatch.

\subsection{Concluding remarks}

In this chapter, we have addressed the challenges involved in extracting the response of soft cellular materials in split Hopkinson pressure bar testing set up. Using explicit dynamic corotational beam formulation along with suitable boundary conditions, the state of stress of the cellular specimen has been examined. The effect of intrinsic and extrinsic parameters of cellular specimen are illustrated, and following conclusion can be drawn from the present analysis: 
1. A cellular structure with lower cell size (higher relative density) attains equilibrium stress state quickly and also offers a uniform deformation throughout the specimen. A larger cell size (lower relative density) specimen not only takes longer time for stress equilibration but also fails to maintain equilibrium stress state during the test process.

2. The average number of cells in the specimen length does not dominate the stress equilibration process but, affects the convergence of the stress-strain response. Higher the number of cells in specimen length for a particular relative density, more representative is the dynamic response of a cellular structure. It has been found that a minimum ratio of specimen length to cell size equal to 13.2 offers a representative dynamic stress-strain response. Previously, Gibson and Ashby (1997) suggested a minimum ratio of specimen length to cell size equal to 20 to evaluate the quasi-static elastic behavior of the specimen. Several authors (Chung and Waas, 2002a,b; Zheng et al., 2005a; Li et al., 2007) also adopted the presently found requirement of minimum ratio of specimen length to cell size in their numerical and experimental observations on the dynamical behavior of cellular materials.

3. Higher loading rate severely affects the stress equilibration of the lower relative density cellular structures.

4. Lower impedance mismatch ratio enables higher relative density specimen to achieve the stress equilibration faster whereas, higher impedance mismatch is required for lower relative density specimen. 


\section{Chapter 4}

\section{Microscopic deformation}

\section{mechanisms associated with the}

\section{transient response of soft cellular materials*}

\subsection{Introduction}

The explicit dynamic finite element framework as described in Chapter 2 has been used to simulate the high strain rate response of cellular materials. We have excluded from our model any rate sensitivity the constituent material might possess. Also, we have considered only large deflection and rotation of cell walls as possible deformation mechanisms (characteristic of elastomeric materials) such that the rate effects result-

$*$ Reprinted from Effect of microscopic deformation mechanisms on the dynamic response of soft cellular materials, Vol 42, Issue 2, Siladitya Pal, Spandan Maiti, Ghatu Subhash, Mechanics of Materials, Pages No 118-133, Copyright 2010, with permission from Elsevier (see Appendix G) 
ing from strut bending and buckling can be studied independent of other strain rate sensitive mechanisms. We perform a detailed parametric study to elaborate the effect of loading pattern, cell wall material properties, average cell size and randomness in microstructure, and cell orientation with respect impact directions on the overall dynamic response of this class of materials. A voronoi tessellation technique is employed to generate a variety of cellular structures with a specified randomness in cell size. Average cell size of these microstructures is varied to investigate the effect of cell size on the dynamic response of cellular materials. One end of the specimen is subjected to an axial impact velocity whereas the other end is held against a rigid boundary. Our numerical framework can produce time snapshots of different quantities of interest, such as strain or velocity, for the entire domain thus enabling the investigation of the connection between local deformation response and global constitutive behavior.

The chapter is organized as follows: in Section 4.2, the computational domain along with boundary conditions are described. A thorough discussion of the parametric study is presented in Section 4.3. Of special interest is the effect of pulse rise time, average cell size, cell irregularity, cell orientation and material properties on the deformation evolution in this class of materials. Finally, we close the discussion by drawing conclusions from the current study in Section 4.4 .

\subsection{Problem description}

In the current study, we model the axial impact of the cellular material by considering a two dimensional computational domain representing the specimen. Figure 4.1(a) shows a cellular specimen of length $L$ and depth $D$ with an aspect ratio $\phi=L / D$. This domain is subjected to velocity boundary condition at the impact end, while the velocity at the other end (support end in Figure 4.1(a)) is kept at zero throughout 
the simulations. Traction free boundary condition has been applied on both the top and bottom edges. Impact velocity $v_{a}$ is applied to all the struts at impact end of

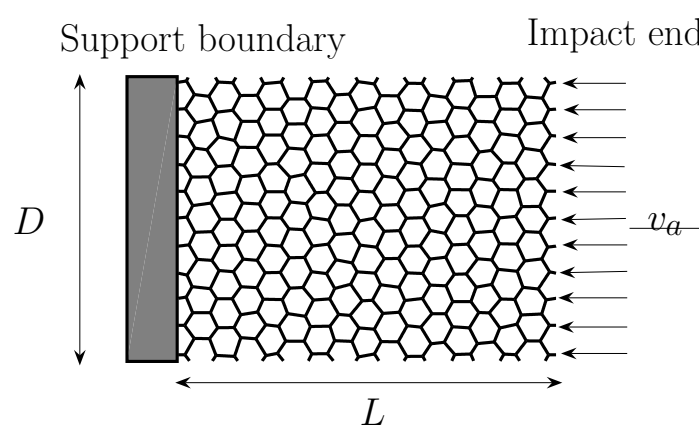

(a)

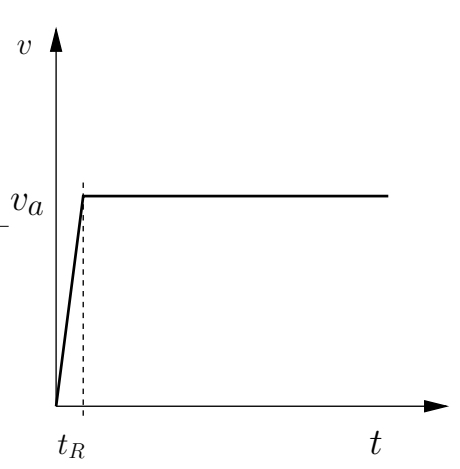

(b)

Figure 4.1: (a) A cellular specimen of length $L$ and depth $D$ is held against a rigid boundary. The specimen is subjected to an impact velocity $v_{a}$ denoted by arrows at the right end. (b) Applied velocity pulse with a magnitude $v_{a}$ and rise time $t_{R}$.

the specimen as shown in the Figure 4.1(a). A typical loading pulse is described in Figure 4.1(b) where $v_{a}$ is the magnitude of the applied velocity and $t_{R}$ is the rise time of the pulse. Strain rate in the specimen can be defined as

$$
\dot{\varepsilon}(t)=\frac{v_{b}-v_{a}}{L},
$$

and nominal strain $\varepsilon$ can be obtained as

$$
\varepsilon(t)=\int_{0}^{t} \dot{\varepsilon}(t) d t
$$

where $v_{b}$ is the velocity of the interface at the support boundary and the specimen. The nominal stress $\sigma$ is calculated from the nodal reactions at the support boundary at the back wall (left end in Figure 4.1(a)). In the following discussion, the terms "stress" and "strain" refer to the nominal stress and nominal strain, respectively. 
Using the numerical scheme described in Chapter 2, deformation behavior of cellular solids is studied for a wide range of microstructures, material properties and loading conditions. A slightly disordered honeycomb microstructure (Figure 2.9(a)), which is essentially two dimensional, has been used for all the simulations presented in this work. Cell size distribution of these microstructures can be described by the mean cell diameter $d_{a v}$ and a standard deviation $S_{d}$, as discussed in Section 2.5. It is assumed that the distribution of the cell size arises from minor processing induced irregularities in the microstructure, and accordingly, a small $S_{d}=0.02 d_{a v}$ is used to characterize all the specimens in the current study (Figure 2.9(b)) except where noted. A specimen of length $L=500 \mu m$ with an aspect ratio $\phi=1$ is chosen for all the subsequent simulations. The simulation domain is so chosen that the ratio of specimen length to cell size varies from 13 to 33. Although, Gibson and Ashby (1997) suggested a minimum ratio of specimen length to cell size equal to 20 to describe the quasi-static elastic behavior of the specimen, the above range of number of cells along the specimen length has been adopted in several numerical and experimental observations on the dynamical behavior of cellular materials (Chung and Waas, 2002a,b; Zheng et al., 2005b; Li et al., 2007). In the present study, it has been found through extensive simulations that specimens with a ratio of specimen length to cell size higher than 13 show similar deformation signatures and associated stress-strain responses. However, for most of the simulations we have kept the cell size fixed at $25.2 \mu \mathrm{m}$, and with a conservative estimate of 20 cells in the length direction, a specimen size $(L)$ of $500 \mu \mathrm{m}$ (and correspondingly, $D=500 \mu \mathrm{m}$ ) is considered as a representative domain for the current study. Specimen cell walls (struts) are assumed to be square in cross-section. The strut thickness is given as $h_{s}=2.5 \mu \mathrm{m}$ and mean cell size $d_{a v}=25.2 \mu \mathrm{m}$ resulting in a relative density (Equation 2.37) of $\bar{\rho}=0.2$. Polyethylene, a polymer whose material properties are given by $E=0.6 \mathrm{GPa}, \rho_{s}=910 \mathrm{kgm}^{-3}$ and $\nu=0.3$ (Gibson and Ashby, 1997), is chosen as the constituent phase of the cellular specimen. It is assumed that the material remains elastic throughout the simulation process though it can exhibit 
considerable geometric nonlinearity in its deformation response. No strain rate effect in the material properties has been assumed in this paper so that we can study the rate effects arising from microinertia and microbuckling only on the dynamic behavior of these materials. Average cell size $d_{a v}$ is varied from 15.1 to $37.5 \mu \mathrm{m}$, while bulk material properties such as Young's modulus and density of constituent material of cellular specimen are also varied systematically.

\subsection{Result and discussions}

Primary goal of the current article is to correlate macroscopic stress-strain response of soft cellular materials with deformation patterns arising from the variation in microstructural as well as material parameters. Investigation of the relative role of microbuckling and microinertia on the evolving deformation pattern is also of special interest. Main results of this study are summarized together in Figure 4.2 and Figure 4.3. In the simulated dynamic stress-strain responses for two different situations shown in Figure 4.2, solid curve is associated with the uniform collapse of cells whereas the dotted curve corresponds to the nonuniform collapse (or progressive collapse) of the cellular specimen, as shown in Figure 4.3. While geometric properties are kept constant for both the specimens, bulk density $\left(\rho_{s}\right)$ for the first case is chosen as 910 $\mathrm{kgm}^{-3}$ and that for the second case is $6825 \mathrm{kgm}^{-3}$ to simulate these two effects in the deformation response. Young's modulus $(E)$ has been fixed at $0.6 \mathrm{GPa}$ for both the simulations.

Observe from Figure 4.2 that for progressive collapse (shown by dashed line in the figure), stress-strain response exhibits an initial high peak followed by a sharp fall in nominal stress. Several experimental observations have shown a similar response (Lee et al., 2006; Vural and Ravichandran, 2003). The initial strength en- 
hancement upto point $P$ in Figure 4.2 occurs due to the effect of microinertia of the struts in the first few layers at the impact end as shown in Figure 4.3(a). This figure clearly shows that deformation is localized in only first one or two layer of cells, and thus, the applied load is borne by the specimen chiefly through its lateral inertia showing an apparent increase in strength that is typical for a dynamic loading scenario. However, due to discrete nature of the microstructure, microbuckling occurs in the struts aligned in the loading direction and starts to rotate the cells at the impact layer (Figure 4.3(a)). This realignment of load bearing elements washes out the inertial stress enhancement leading to a sharp drop in stress (indicated by point $Q)$. Correspondingly, sudden collapse of first few layers of cells due to microbuck-

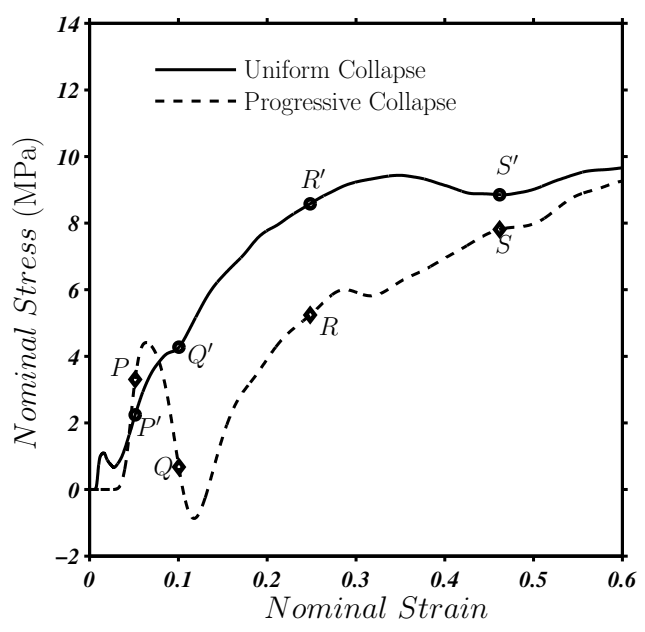

Figure 4.2: Stress-strain response for two different collapse modes: uniform collapse indicated by solid line, and progressive collapse denoted by dashed line. Bulk density of the material is taken as $910 \mathrm{kgm}^{-3}$ for the first case, while that for the second case is $6825 \mathrm{kgm}^{-3}$. Young's modulus for both the materials are fixed at $0.6 \mathrm{GPa}$, while dimension of the specimen for both the cases are also held constant.

ling can be observed. Further increase in external loading progressively recruits more cells from next layers in the deformation zone to resist the loading (points $R$ and $S)$. Stiffening behavior of already collapsed cells, which start to behave like a solid material, along with the softening response associated with the collapse of new rows of cells further from the impact end are responsible for the continued increase in stress 
during the rest of the deformation. No actual plateau region can be observed in the "constitutive behavior" for this case as more and more cell layers are drawn into the severely localized deformation zone as shown in Figure 4.3(a).

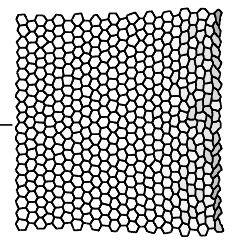

$P$

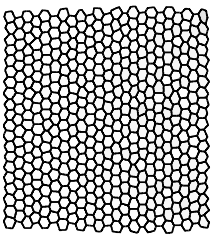

$P^{\prime}$

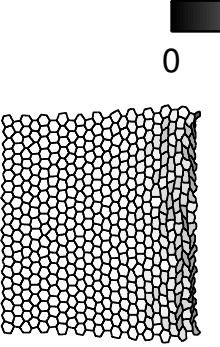

$Q$
$0.5 \quad 1$

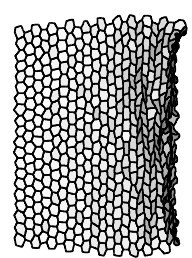

$R$

(a)

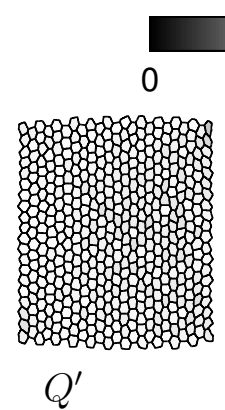

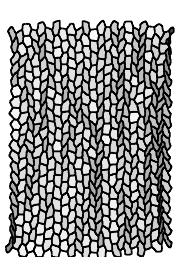

$R^{\prime}$

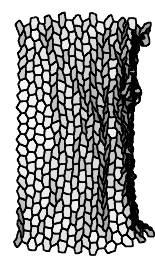

$S$

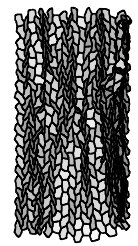

$S^{\prime}$

(b)

Figure 4.3: Snapshots of deformed specimens for (a) progressive collapse and (b) uniform collapse. Degree of deformation in each cell is represented by a gray scale varying from 1 (no deformation) to 0 (full collapse).

On the other hand, in case of uniform deformation (shown by solid line in Figure 4.2), no collapse of cells can be observed in the immediate vicinity of the impact end (Figure 4.3(b)) for most of the deformation history. In contrast to the first case, all the cells in the specimen deforms simultaneously giving rise to the gradually increasing stress response as indicated by points $Q^{\prime}$ and $R^{\prime}$ in Figure 4.2. However, at point $S^{\prime}$, all the cell layers in the specimen starts collapsing together due to the combined effect of microbending and microbuckling of cell walls so that no further increase in stress carrying capacity is possible. This situation gives rise to the plateau region (point 
$\left.S^{\prime}\right)$ in the nominal stress-strain response. Although the above features are seen for two different foam bulk densities, deformation signatures and associated stress-strain responses are also dependent on microstructural features, other material properties and loading parameters as will be discussed in subsequent sections.

\subsubsection{Effect of pulse rise time}

In this section, the influence of rise time $t_{R}$ (see Figure 4.1(b)) of the applied velocity on the deformation behavior of a cellular specimen is discussed. The specimen is held against a rigid boundary and the other end is subjected to a wide range of impact velocities varying from $v_{a}=2.5$ to $125 \mathrm{~ms}^{-1}$ resulting in strain rates ranging from $\dot{\varepsilon}=5.0 \times 10^{3}$ to $2.5 \times 10^{5} \mathrm{~s}^{-1}$. For each loading case, the rise time $t_{R}$ is varied from 0.3 to $9.3 \mu \mathrm{s}$.

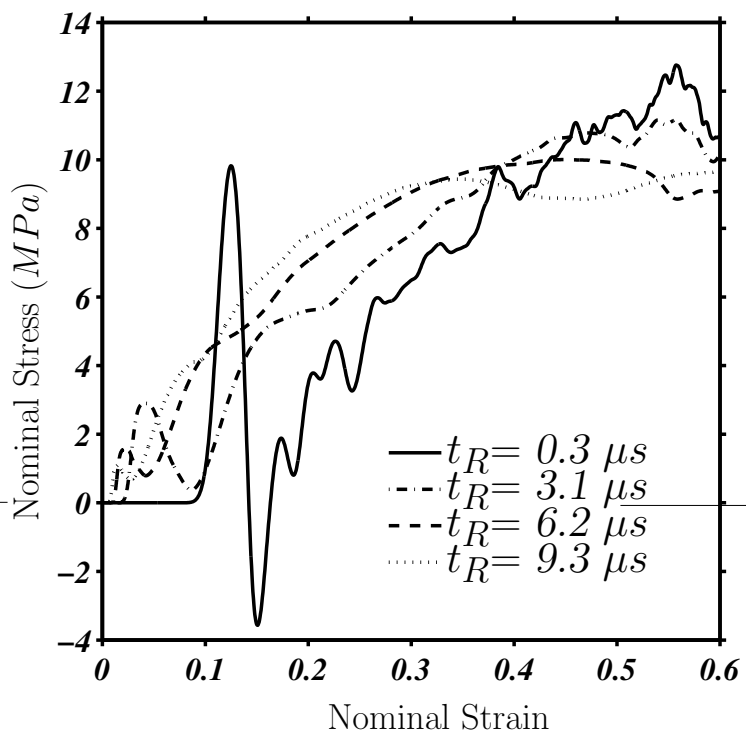

(a)

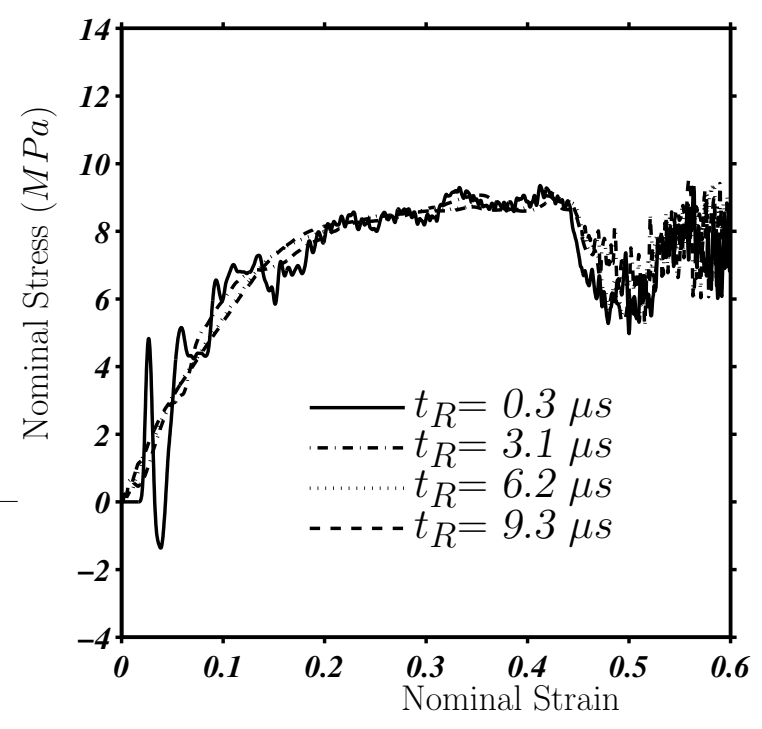

(b)

Figure 4.4: Stress-strain responses of a cellular material with different pulse rise times at (a) $\dot{\varepsilon}=1.0 \times 10^{5} \mathrm{~s}^{-1}$ and (b) $\dot{\varepsilon}=2.0 \times 10^{4} \mathrm{~s}^{-1}$. 
The nominal stress-strain responses of the cellular specimens under study at a strain rate $\dot{\varepsilon}=1.0 \times 10^{5} \mathrm{~s}^{-1}$ and different pulse rise times are depicted in Figure $4.4(\mathrm{a})$. This figure reveals that when the pulse rise time is short (as example, $t_{R}=0.3 \mu s$ ), an early spike is observed in the stress-strain response. Such a stress overshoot is associated with the microinertial effect as explained earlier (Figure 4.2) that arises due to a sudden application of the impact velocity $v_{a}$. Sharp fall in stress following this overshoot is accompanied by the local collapse of front layer of cells at the impact end due to microbuckling of struts. However, gradual increase in pulse rise time results in a gradual reduction of the magnitude of the initial stress spike as seen Figure 4.4(a). This observation can be attributed to the decrease in the inertial force due to lower acceleration associated with increased $t_{R}$. It can also be noticed that stress overshoot occurs at decreasing nominal strain levels as the rise time increases. This behavior is due to the fact that the cells at the impact end of the specimen undergoes highly localized deformation thus delaying the arrival of stress wave at the back (support) wall where the stress is measured. Also note that the specimen with the shortest rise time shows a highly oscillatory stress-strain response. However, these fluctuations reduce as the rise time increases, a phenomenon also observed by Hönig and Stronge (2002a), and can be explained to be arising from local cell collapse.

The cellular material subjected to a lower strain rate $\left(\dot{\varepsilon}=2.0 \times 10^{4} s^{-1}\right)$ shows a different stress-strain response, see Figure 4.4(b). The response at the shortest rise time, i.e., $t_{R}=0.3 \mu \mathrm{s}$, shows early oscillations but of significantly lower amplitude than those associated with the same rise time in Figure 4.4(a). This observation can be attributed to the fact that, initial acceleration associated with the previous case is appreciably higher than that for the current case due to a higher final velocity applied in the former case. This increased acceleration of the applied velocity solicits a higher inertial response resulting in much increased oscillations in the stress-strain curve. This argument can be further reinforced from the fact that all other responses shown in Figure 4.4(b) overlay on each other indicating that the stress-strain curves 
are not significantly altered by the pulse rise time at the lower strain rate regime due to low initial acceleration. Such superposed stress-strain responses for different pulse rise times also indicate that the cellular specimen experiences similar modes of deformation for all these cases. Observe that there is no delay associated with stress signal (measured on the backwall of the specimen) as was found previously for the shortest rise time at a higher strain rate (i.e., for $t_{R}=9.3 \mu \mathrm{s}$ in Figure 4.4(a)). This fact implies that the stress wave reaches the backwall before any appreciable local deformation has occurred at the impact end.

Figure 4.5 illustrates the variation of plateau stress $\sigma_{p l}$ for different strain rates and rise times discussed above. The plateau stress $\sigma_{p l}$ is calculated as the stress at a strain $\varepsilon=0.4$. It can be found that for the strain rates below $2.5 \times 10^{5} \mathrm{~s}^{-1}$, the plateau stress remains essentially constant for different rise times while increasing with strain rate for a given rise time. However, at $\dot{\varepsilon}=2.5 \times 10^{5} \mathrm{~s}^{-1}$, the plateau stress exhibits a gradual increase with rise time while mostly staying below other cases.

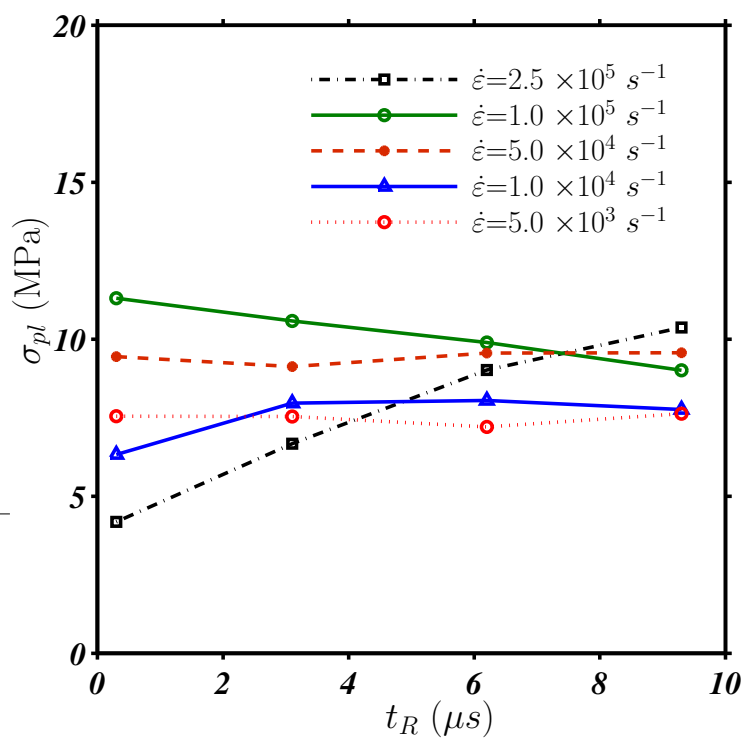

Figure 4.5: Plateau stress $\sigma_{p l}$ for different pulse rise times and strain rates.

To understand the origin of such behavior, as well as the oscillations in stress-strain 


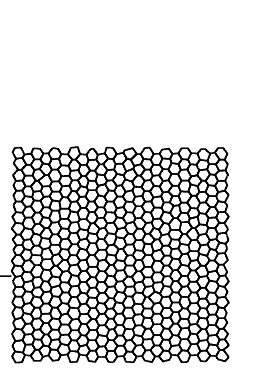

$\varepsilon=0$

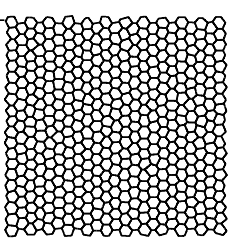

$\varepsilon=0$

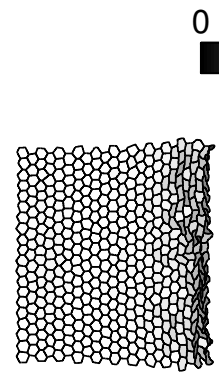

$\varepsilon=0.1$

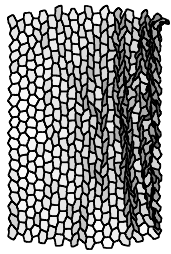

$\varepsilon=0.3$

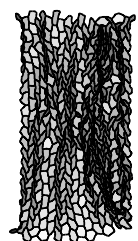

$\varepsilon=0.47$

(a)

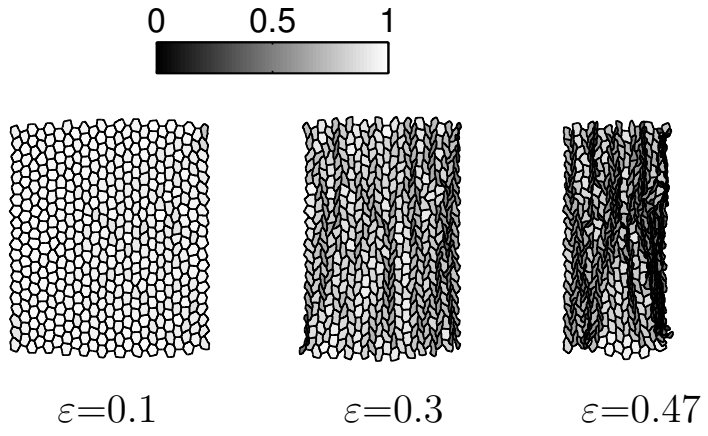

(b)

Figure 4.6: Snapshots of deformed specimens at selected strain intervals for (a) $t_{R}=0.3 \mu \mathrm{s}$ and (b) $t_{R}=9.3 \mu \mathrm{s}$ at a strain rate $\dot{\varepsilon}=1.0 \times 10^{5} \mathrm{~s}^{-1}$. The cellular material is made of polyethylene with $d_{a v}=25.2 \mu \mathrm{m}$. Degree of deformation in each cell is represented by a gray scale varying from 1 (no deformation) to 0 (full collapse).

curves shown in Figure 4.4(a), we focus our attention towards the "snapshots" of the deformed specimen at different times. Figure 4.6 shows snapshots of the deformation evolution at selected strain intervals in a cellular specimen for a strain rate of $\dot{\varepsilon}=1.0 \times 10^{5} \mathrm{~s}^{-1}$ and for pulse rise times $t_{R}=0.3 \mu \mathrm{s}$ and $t_{R}=9.3 \mu \mathrm{s}$, respectively. For clarity, the degree of deformation in each cell is represented by a linear gray scale with ' 1 ' representing the undeformed cell (colored white) and ' 0 ' indicating a fully collapsed cell (colored dark). However, we did not assign a gray scale for incomplete cells at the boundaries, and consequently, they show in white in all the figures. For 
$t_{R}=0.3 \mu \mathrm{s}$, it can be seen from Figure $4.6(\mathrm{a})$ that a collapse band is initiated at an early stage of $\varepsilon=0.1$ at the impact end whereas the rest of the domain remains undeformed. With such a short pulse rise time, the cell walls along first few layers at the impact end almost immediately reach the critical buckling load of the struts (preceded by microinertial stress enhancement and associated peaks in stress-strain curves as seen in Figure 4.4). This local deformation occurs well before the stress wave can propagate to the support boundary where it is measured. Therefore, no significant stress can be detected at the back end although the specimen has undergone appreciable amount of deformation at the impact end. After the initiation of the narrow collapsed zone, the localized deformation zone progressively extends towards the support boundary. Such a nonuniform mode of deformation along with the progressive collapse of layers of cells in the cellular specimen leads to an oscillatory stress-strain response as was observed in Figure 4.4(a). On the other hand, with a longer rise time of $t_{R}=9.3 \mu \mathrm{s}$, different deformation characteristics emerge for the same specimen as seen in Figure 4.6(b) where no localized bands originates at the early stage of impact $(\varepsilon=0.1)$. This fact can be attributed to a lower initial velocity due to long rise time and corresponding low stress rise in the struts. The deformation of the specimen is chiefly governed by microbending of cell walls rather than microbuckling. At $\varepsilon=0.3$, unlike the highly deformed bands at the impact end in case (a) of Figure 4.6, the deformed bands with lesser intensity are seen throughout the specimen, i.e., the deformation is more uniform. Such a uniform deformation mode produces a smoother stress-strain response compared to the localized collapse seen for the previous case. However, for still larger deformation, i.e., at $\varepsilon=0.47$, both the deformation snapshots for $t_{R}=0.3 \mu \mathrm{s}$ and $t_{R}=9.3 \mu \mathrm{s}$ are similar. Thus, although the deformation histories are different, the final deformation patterns are almost similar and accordingly the average plateau stresses $\sigma_{p l}$ are equal above a nominal strain of 0.4 for both the cases as shown in Figure 4.4(a). This fact is also reflected in Figure 4.5 where the plateau stress remains essentially constant with rise time for strain 
rates lower than $1.0 \times 10^{5} \mathrm{~s}^{-1}$. However, at still higher strain rates, more number of layers collapse immediately after the impact as the rise time decreases resulting in a lower overall plateau stress. It should be noted here that the rise time of the loading pulse affects the stress equilibration of soft materials such as polymeric foams in split Hopkinson pressure bar test. Similar to the above observation, a short rise time of loading pulse leads to a nonhomogeneous deformation whereas a longer rise time leads to a more uniform deformation during SHPB testing of polymeric materials (Chen et al., 2002b). A nonhomogeneous deformation restricts the specimen from attaining the stress equilibration and leads to an inaccurate "stress-strain" response in a classical SHPB set up. In order to circumvent the above difficulties, a pulse shaping techniques are generally used which essentially increase the rise time of the incident pulse (Frew et al., 2002; Subhash and Ravichandran, 2000b).

\subsubsection{Effect of cell size}

To study the effect of cell size on the deformation behavior of cellular materials, average cell size $d_{a v}$ of the specimen is varied from 15.1 to $37.5 \mu \mathrm{m}$ while cell wall (strut) thickness $h_{s}$ is kept constant at $2.5 \mu \mathrm{m}$. Such a variation results in a range of relative density $\bar{\rho}$ varying from 0.35 to 0.14 . As the bulk material density of constituent material remains constant, i.e., $\rho_{s}=910 \mathrm{kgm}^{-3}$, the apparent density of the cellular specimen $\rho$ (defined by Equation 2.37) varies from 317.0 to $126.1 \mathrm{kgm}^{-3}$. The specimen is supported against a rigid boundary and is subjected to different axial impact velocities ranging from $v_{a}=2.5$ to $125 \mathrm{~ms}^{-1}$ (with attendant strain rates of $5.0 \times 10^{3}$ to $2.5 \times 10^{5} \mathrm{~s}^{-1}$ ) with a fixed pulse rise time $t_{R}=9.3 \mu \mathrm{s}$. Stress-strain responses of cellular specimens with different average cell sizes at a strain rate of $\dot{\varepsilon}=1.0 \times 10^{5} \mathrm{~s}^{-1}$ are illustrated in Figure 4.7. Corresponding apparent densities are also noted in the figure legend. It can be noticed that with decreasing cell size, the 


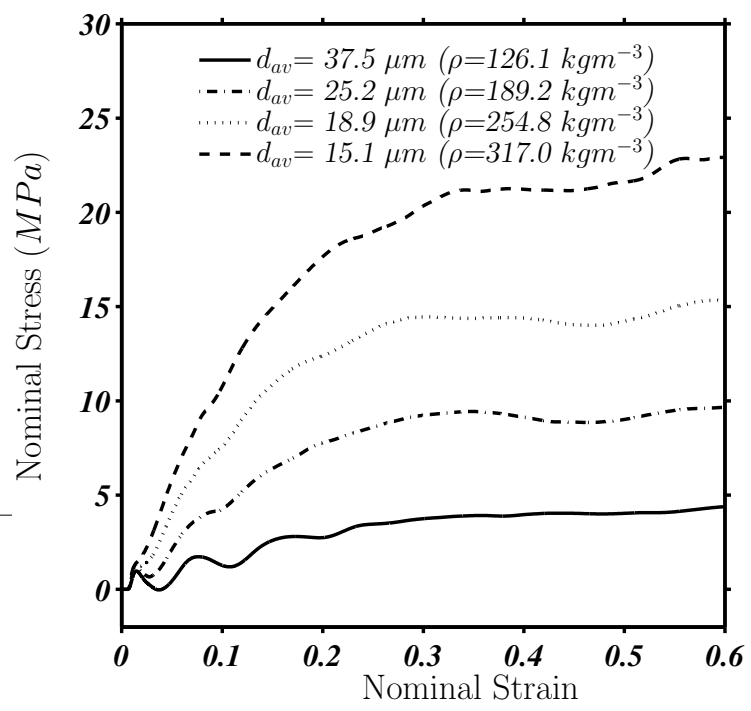

Figure 4.7: Stress-strain behavior of a cellular material with different cell sizes $d_{a v}$ and, equivalently, apparent densities $\rho$ at a strain rate $\dot{\varepsilon}=1.0 \times 10^{5}$ $s^{-1}$ and pulse rise time $t_{R}=9.3 \mu s$.

specimen exhibits a stiffer stress-strain response and a higher plateau stress. As the cell size increases, the bending as well as buckling strength of the struts decreases rapidly due to an increase in effective length of these structural elements. This decrease in strength results in early collapse of cells and manifests as an increasingly compliant stress-strain response with reduced plateau stress. A similar dependency of plateau stress on the density of cellular specimen has also been observed experimentally by Vural and Ravichandran (2003) and Saha et al. (2005).Variation of plateau stress with respect to cell size $d_{a v}$ and square of cell size $d_{a v}^{2}$ (which is measure of cell area) are illustrated in the Figure 4.8. It can be seen that as the cell size increases, the plateau stress decreases rapidly. Brezny and Green (1990) also experimentally found that the crushing strength of a brittle reticulated vitreous carbon foam varied inversely with cell size $d_{a v}$. Our analysis indicates that the plateau stress obeys the inverse relationship more accurately with the cell area (Figure 4.8(b)) rather than the cell size as found by these authors. Also observe from Figure 4.8 that the scatter between the points at a particular cell size is high at low cell size, while there is almost no scatter at higher cell sizes. This observation leads to the fact that, the effect of strain rate 


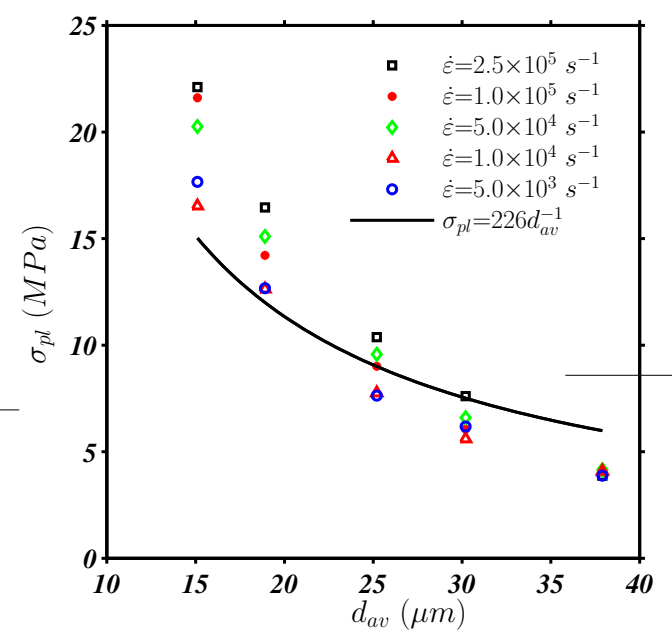

(a)

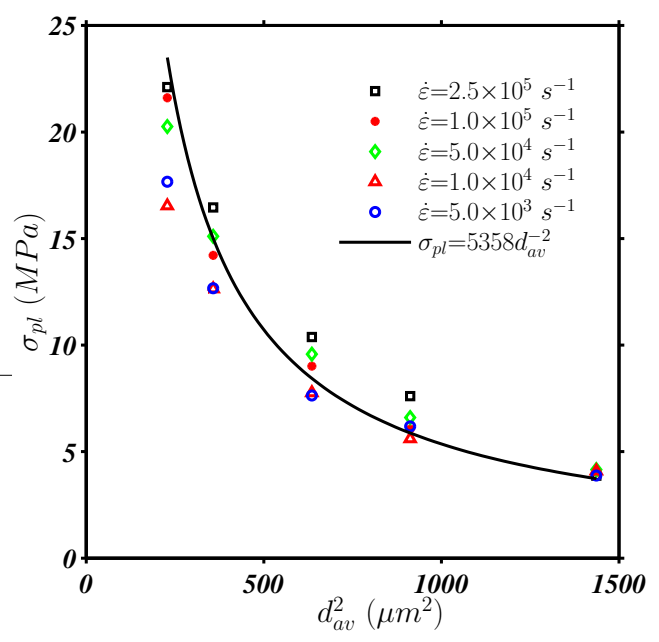

(b)

Figure 4.8: Plateau stress for cellular specimens with (a) cell sizes and (b) square of cell sizes when subjected to different strain rates. The pulse rise time is fixed at $t_{R}=9.3 \mu \mathrm{s}$.

is more pronounced at low cell sizes. Our study suggest that as the density increases (cell size decreases), plateau stress increases linearly with the strain rate on a logarithmic scale. An experimental investigation conducted by Tedesco et al. (1993) on polyurethane foams also confirms a similar trend for different strain rates. Saha et al. (2005), Vural and Ravichandran (2003) and Subhash et al. (2006b) also found low strain rate sensitivity of $\sigma_{p l}$ at low density and high rate sensitivity of $\sigma_{p l}$ at high density of foams, consistent with our simulation results. It can be inferred that, at least a part of this rate sensitivity stems from the microstructural properties of the cellular materials that manifests itself through the inertial effects as well as buckling and bending of the cell wall at the microstructure level.

It can be concluded from the previous discussion that plateau stress depends greatly on the cell size (or density) of the specimen. In order to correlate the deformation initiation and propagation with the plateau behavior, local strains are calculated over time. In the present study, the deformation patterns remain almost similar along specimen depth $D$ (see Figure 4.1(a)). Hence, local strain is calculated on a narrow 
window extended along the length $L$ in the middle of the cellular specimen. Figure 4.9 shows the evolution of local strain along the specimen length with time for two different cellular materials with $d_{a v}=37.5 \mu \mathrm{m}$ and $d_{a v}=18.9 \mu \mathrm{m}$. The parameter $\xi$ denotes the distance along the specimen, with support and impact ends represented by $\xi=0$ and $\xi=L$, respectively. Local strain in the specimen is defined as $\varepsilon_{l}=1-A_{c} / A_{0}$, where $A_{c}$ and $A_{0}$ are the current and initial areas of a cell, respectively. A similar technique was also introduced by Voit et al. (2007) to experimentally measure the local strain of polymeric foam under impact loading. Both the specimens depicted in Figure 4.9

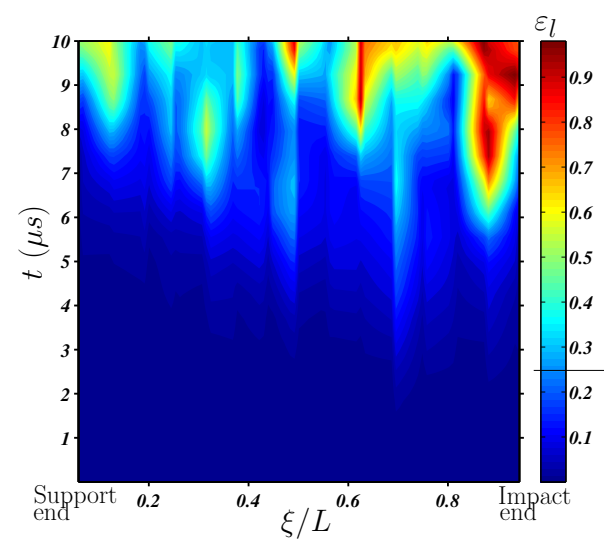

(a)

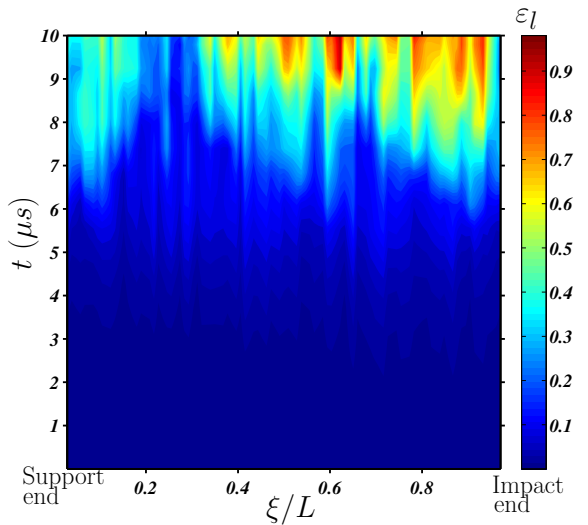

(b)

Figure 4.9: Evolution of local strain $\varepsilon_{l}$ with time along specimen length for (a) $d_{a v}=37.5 \mu \mathrm{m}$ and (b) $d_{a v}=18.9 \mu \mathrm{m}$ at a strain rate $\dot{\varepsilon}=1.0 \times 10^{5} \mathrm{~s}^{-1}$ and $t_{R}=9.3 \mu \mathrm{s}$. The distance of any point along specimen length from the rigid boundary is denoted by $\xi$, where $\xi=0$ and $\xi=L$ specify support and impact ends, respectively.

show that the deformation localization occurs quite late in the loading history (after $7 \mu s$ for both the cases). While an almost uniform deformation localization can be observed for case (a), case (b) exhibits a narrow localized zone at the impact end.

The above modes of deformation evolution for two different cell sizes can be further illustrated with the help of deformation snapshots. Figure 4.10 illustrates the snapshots of two specimens with cell size $d_{a v}=37.5 \mu \mathrm{m}$ and $d_{a v}=18.9 \mu \mathrm{m}$. At $t=6.7 \mu \mathrm{s}$ $(\varepsilon=0.25)$, although deformed cells can be found throughout the domain, a few layers 

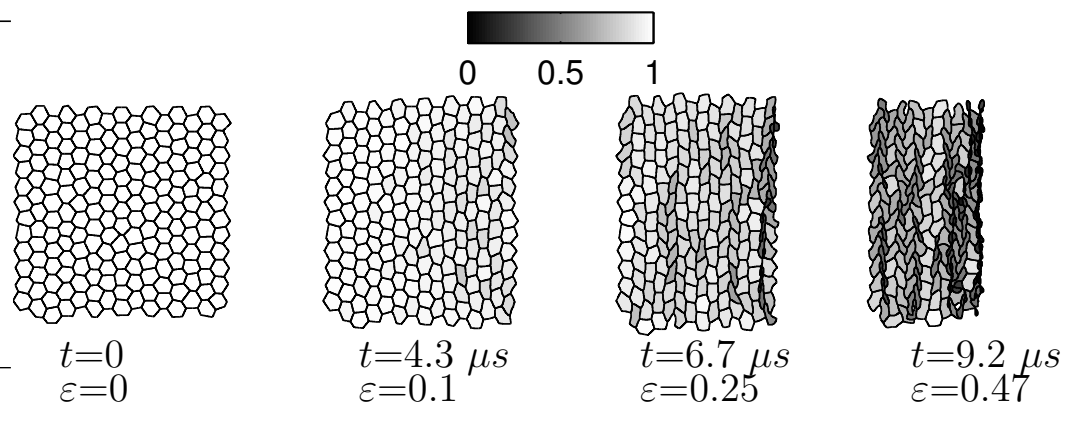

(a)
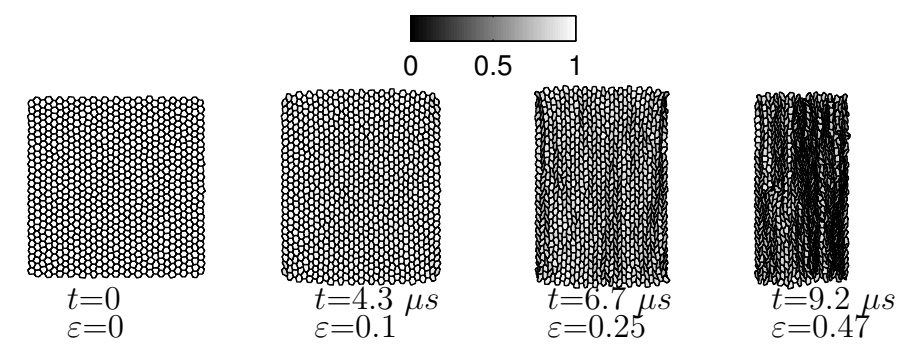

(b)

Figure 4.10: Snapshots of deformed specimens at selected time intervals for (a) $d_{a v}=37.5 \mu \mathrm{m}$ and (b) $d_{a v}=18.9 \mu \mathrm{m}$ at a strain rate $\dot{\varepsilon}=1.0 \times 10^{5} \mathrm{~s}^{-1}$ with $t_{R}=9.3 \mu \mathrm{s}$. Degree of deformation in each cell is represented by a gray scale varying from 1 (no deformation) to 0 (full collapse).

of cells have almost collapsed at the impact end for the specimen with $d_{a v}=37.5 \mu \mathrm{m}$ (Figure 4.10(a)) whereas the smaller cell size specimen shows a more uniformly distributed deformed zone throughout the specimen (Figure 4.10(b)). A larger cell size is more susceptible to buckling compared to smaller cell size owing to its lower buckling strength. Note that specimen with smaller cell size exhibits predominant bending but negligible buckling of cell walls upto a strain of 0.25 . Moreover, microinertia effect for all the cases (see Figure 4.7) is almost similar as the lateral inertia (mass of the struts) does not vary significantly with average cell size. This observation explains the localized failure behavior as seen from Figure 4.10, low plateau stress (Figure 4.7) for low density specimens, as well as the presence of inertial stress enhancement of almost similar magnitude for all the cases studied. In conclusion, the current study clearly reveals that localized deformation band initiation and propagation under high strain rate loading greatly depends on the average cell size of cellular solids, and the 
underlying mechanisms may be microbuckling and microbending of cell walls.

\subsubsection{Effect of material properties}

The effect of material properties are studied in this section through a systematic variation of Young's modulus and bulk density of the constituent material. The specimen is subjected to impact velocities ranging from $v_{a}=2.5$ to $125 \mathrm{~ms}^{-1}$ with a pulse rise time $t_{R}=9.3 \mu \mathrm{s}$ resulting in strain rate variation between $\dot{\varepsilon}=5.0 \times 10^{3}$ to $2.5 \times 10^{5} s^{-1}$.

\subsubsection{Effect of Young's modulus}

For all the simulations in this section, density of the bulk material $\rho_{s}$ is kept constant at $910 \mathrm{kgm}^{-3}$ whereas the Young's modulus $E$ is varied from 0.15 to $1.5 \mathrm{GPa}$. Figure 4.11 shows the corresponding stress-strain responses at a strain rate $\dot{\varepsilon}=1.0 \times 10^{5}$ $s^{-1}$ and pulse rise time $t_{R}=9.3 \mu s$. Observe that, as the Young's modulus of the cellular material increases, the specimen offers increasingly stiffer stress-strain response. For all the cases except $E=1.5 G P a$, a well defined plateau region is achieved after the initial oscillations due to the inertial effect. Nominal strain corresponding to the initiation of the plateau region also increases with the Young's modulus. However, stress-strain response for the case of $E=1.5 \mathrm{GPa}$ exhibits a continuously stiffening behavior without a well defined plateau region.

Figure 4.12 shows the variation of plateau stress for different Young's moduli at various strain rates. It can be seen that plateau stress increases linearly with Young's modulus for all strain rates. Plateau stress is almost the same at all strain rates for lower modulus specimens considered in this study. However, the strain rate effect 


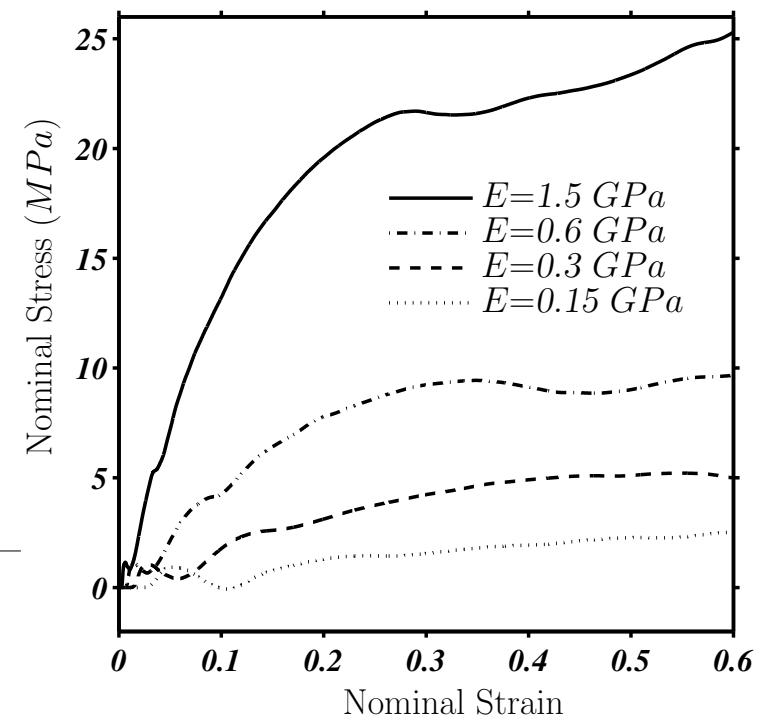

Figure 4.11: Stress-strain response of a cellular material for different Young's moduli at a strain rate $\dot{\varepsilon}=1.0 \times 10^{5} \mathrm{~s}^{-1}$ and for pulse rise time $t_{R}=9.3$ $\mu \mathrm{s}$. The cell size of the specimen is $d_{a v}=25.2 \mu \mathrm{m}$.

becomes apparent at higher Young's modulus, and a clear increase in plateau stress corresponding to an increase in strain rate can be observed from this figure.

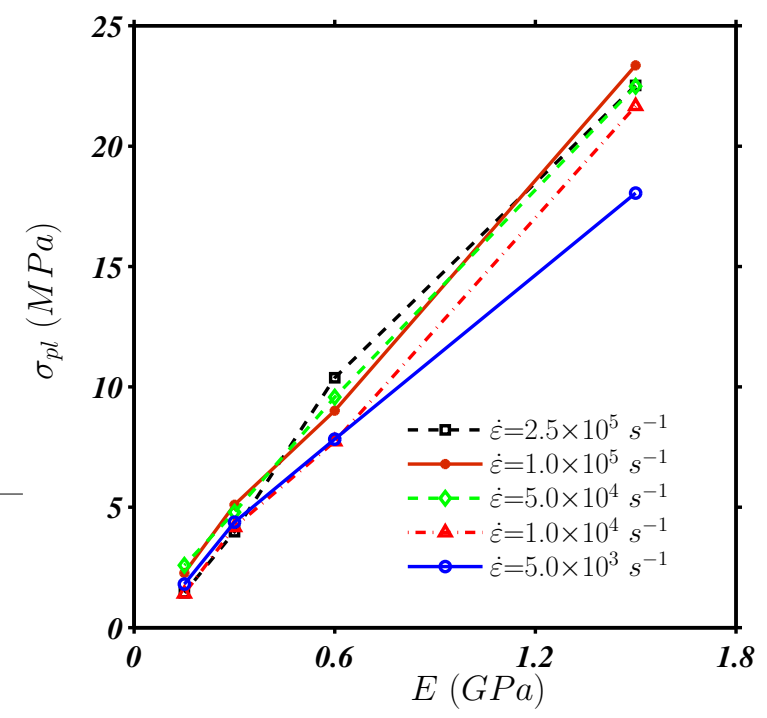

Figure 4.12: Plateau stress for cellular specimens with various Young's moduli when subjected to different strain rates. The pulse rise time is fixed at $t_{R}=9.3 \mu \mathrm{s}$.

Figure 4.13 compares deformed snapshots of the two specimens with different Young's 

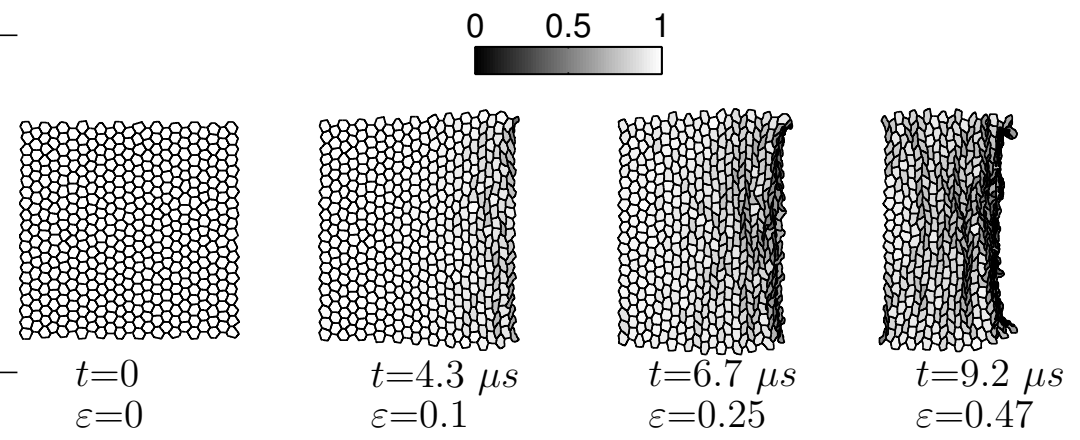

(a)
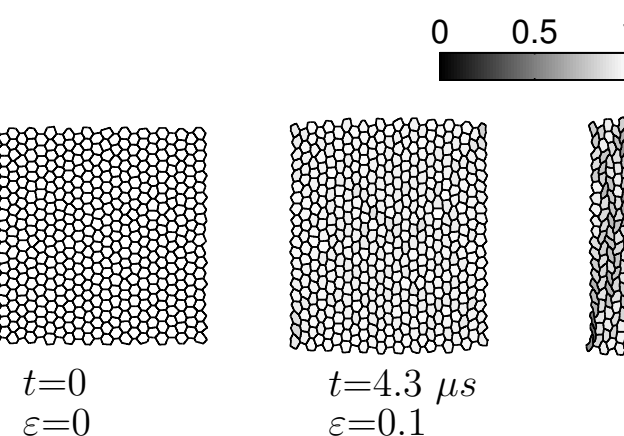

$t=0$

$\varepsilon=0$

$\varepsilon=0.1$
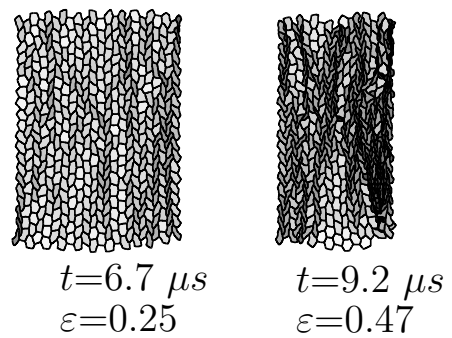

(b)

Figure 4.13: Snapshots of deformed specimens at selected time intervals for (a) $E=0.15 \mathrm{GPa}$ and (b) $E=1.5 \mathrm{GPa}$ at a strain rate $\dot{\varepsilon}=1.0 \times 10^{5} \mathrm{~s}^{-1}$ with $t_{R}=9.3 \mu \mathrm{s}$. Degree of deformation in each cell is represented by a gray scale varying from 1 (no deformation) to 0 (full collapse). The average cell size of the specimen $d_{a v}=25.2 \mu \mathrm{m}$.

moduli. At $t=4.3 \mu \mathrm{s}(\varepsilon=0.1)$, the figure shows the presence of collapsed cells only at the impact end for the specimen with $E=0.15 \mathrm{GPa}$ (Figure 4.13(a)) whereas no significant deformed bands can be found in the domain for $E=1.5 \mathrm{GPa}$ (Figure 4.13(b)). For a fixed strut geometry, the critical buckling load is proportional to $E$. Consequently, a cellular material with lower modulus possesses a lower critical load causing early collapse at the impact end. Due to the higher buckling load, no collapse can be found for cellular material with $E=1.5 \mathrm{GPa}$. At $t=6.7 \mu s(\varepsilon=0.25)$, when several wave reflections have occurred in the specimen, the cellular material with higher modulus shows deformation distributed throughout the entire domain. The deformation occurs mainly due to the microbending of the struts. It can be concluded here 
that Young's modulus plays an important role in determining microbuckling and microbending events in the cellular specimen thus affecting overall deformation behavior and corresponding nominal stress-strain response.

\subsubsection{Effect of bulk material density}

In this section, effect of the bulk material density $\rho_{s}$ on deformation response is characterized. Bulk material density $\rho_{s}$ is varied from 910 to $9100 \mathrm{kgm}^{-3}$ for a fixed cell size specimen $\left(d_{a v}=25.2 \mu \mathrm{m}\right)$ while Young's modulus $E$ of the material is kept constant at $0.6 \mathrm{GPa}$. Figure 4.14 shows the stress-strain response at a strain rate $\dot{\varepsilon}=1.0 \times 10^{5} \mathrm{~s}^{-1}$ and for $t_{R}=9.3 \mu \mathrm{s}$. It is interesting to note that a cellular specimen with lower material density experiences stress throughout the specimen quickly with a small initial bump and then stabilizes with strain. As the density of the material increases, there is a larger delay before the stress is sensed at the support end of the specimen. The amplitude of the initial peak also increases dramatically with density but the stress level after these peaks decreases rapidly with increasing density. Also note that the lower density specimen shows a smooth rise in stress whereas a higher density specimen shows an oscillatory response. The reasons behind such responses will be presented with the help of deformation evolution snapshots in the subsequent discussion.

Figure 4.15 presents the evolution of local strain along the specimen length for $\rho_{s}=910$ $\mathrm{kgm}^{-3}$ and $\rho_{s}=6825 \mathrm{kgm}^{-3}$. It can be seen from Figure 4.15(a) that uniform local strain initiates for lower density cellular material and continues till the end of the simulation (nominal strain $=0.6$ ). On the other hand, for the higher density cellular material, localized crushing (strongest among all the cases studied earlier in this section) occurs at the impact end (Figure 4.15(b)). It is also evident that local strain commences much earlier in time for a higher density specimen. Also, the impact 


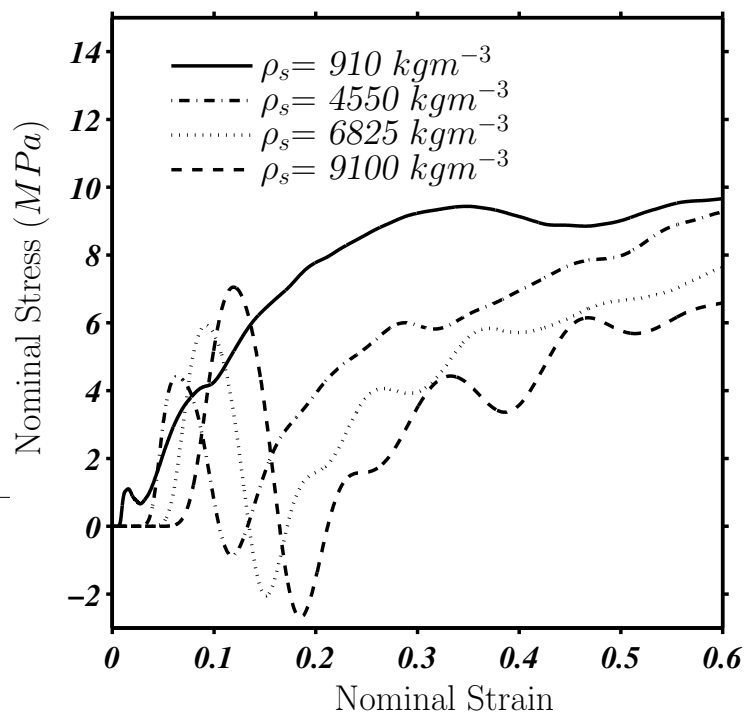

Figure 4.14: Stress strain response of a cellular material with different bulk material densities at a strain rate $\dot{\varepsilon}=1.0 \times 10^{5} \mathrm{~s}^{-1}$ and $t_{R}=9.3 \mu \mathrm{s}$. The cell size of the specimen given as $d_{a v}=25.2 \mu \mathrm{m}$.

end deforms substantially before stress wave reaches the other boundary causing the delay in the stress-strain response in Figure 4.14. The presence of highly localized

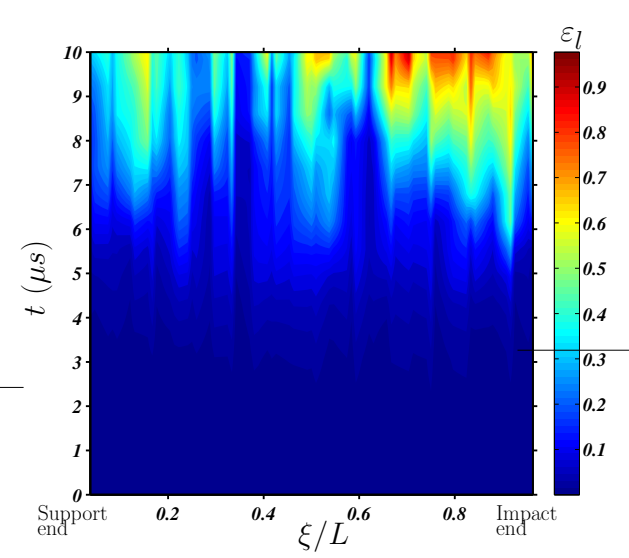

(a)

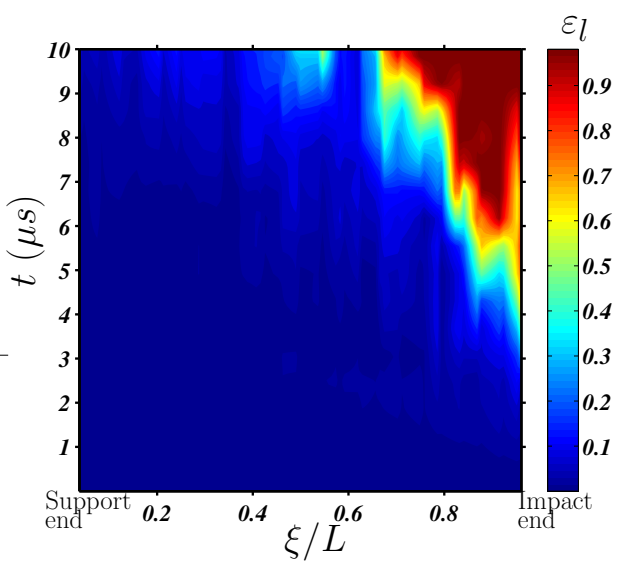

(b)

Figure 4.15: Evolution of local strain with time along specimen length for (a) $\rho_{s}=910 \mathrm{kgm}^{-3}$ and (b) $\rho_{s}=6825 \mathrm{kgm}^{-3}$ at a strain rate $\dot{\varepsilon}=1.0 \times 10^{5} \mathrm{~s}^{-1}$ and $t_{R}=9.3 \mu \mathrm{s}$. The distance of any point along specimen length from the support boundary is denoted by $\xi$, where $\xi=0$ and $\xi=L$ specify support and impact end respectively.

deformation for higher density cellular material leads to an oscillatory stress-strain 
response as found in Figure 4.14. In contrast, for a lower density specimen, a homogeneously distributed strain prevails along the specimen length giving rise to a smooth stress-strain response. It is also evident from the stress-strain response that delay in stress signal at the backwall is much lower for lower density specimens.

Figure 4.16 compares deformed snapshots of the above two cellular materials. Figure 4.16(b) clearly shows the early presence of a row of collapsed cells at the impact end for the higher density cellular material. The cellular material with higher density offers higher microinertial resistance to the deformation causing a high stress enhancement (an initial peak in the stress-strain response) as explained before in Figure 4.2. After the initial stress enhancement, the microbuckling of the struts at the impact end takes place reducing the stress value immediately to zero followed by an increasing stress response with continued loading. However, the lower density cellular material does not exhibit such localized deformation at this stage $(\mathrm{t}=4.3 \mu \mathrm{s}$ or $\varepsilon=0.1)$. At $\mathrm{t}=6.7 \mu \mathrm{s}(\varepsilon=0.25)$, the narrow collapse band continues to grow at the impact end for the higher density specimen while the cells at the support end remain undeformed. On the contrary, for the lower density specimen, it appears that the deformation is distributed uniformly throughout the specimen. Even at $\mathrm{t}=9.2 \mu \mathrm{s}$ $(\varepsilon=0.47)$, the deformation is still highly localized at the impact end for the higher density specimen, but scattered localized bands are found throughout the domain in the lower density specimen. Because buckling and bending strengths remain unaltered in both specimens, (as Young's modulus, cross section of struts and average cell size remain constant throughout), it can be inferred that the deformation localization in high density cellular material is primarily due to microinertial effect. Also, these simulations conclusively reveal that microinertial effect alone accounts for the strong initial stress enhancement in the dynamic "constitutive response" of cellular materials. 

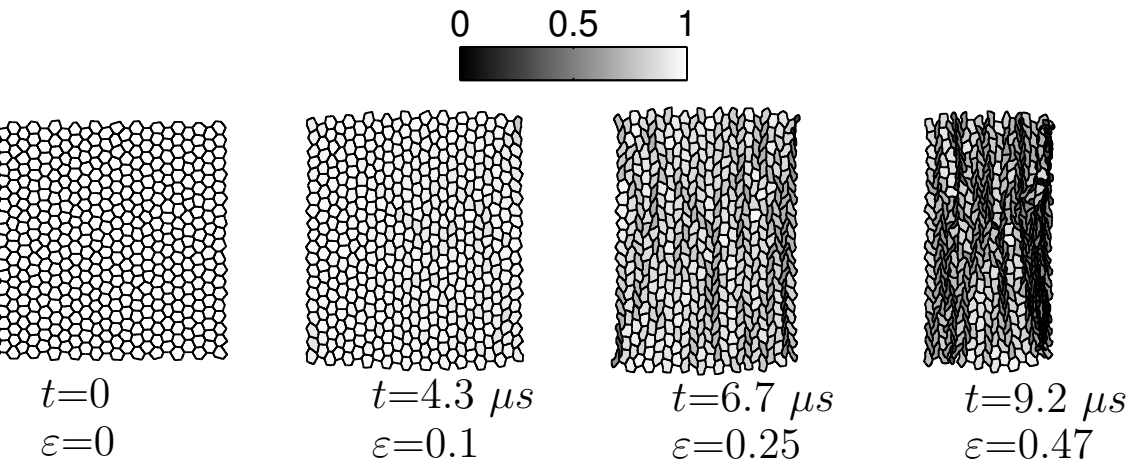

(a)
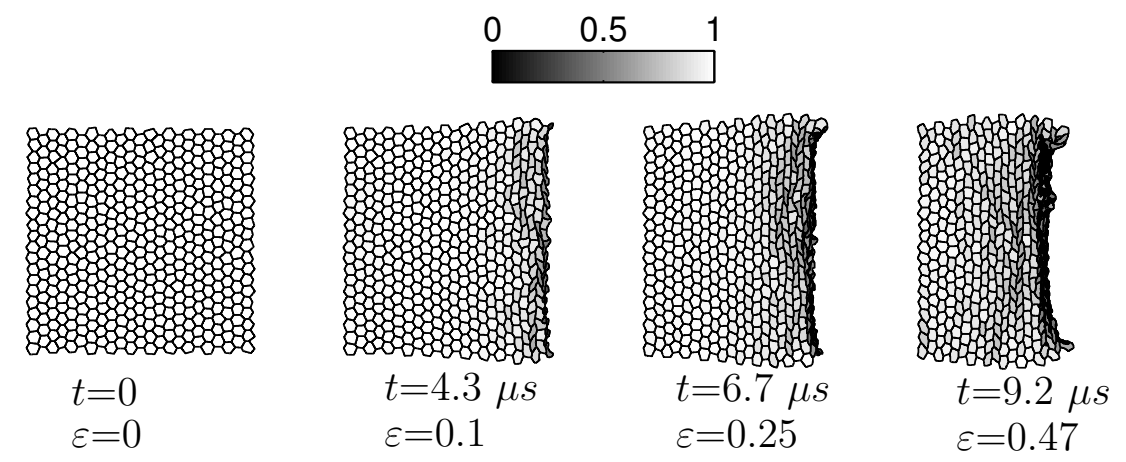

(b)

Figure 4.16: Snapshots of deformed specimens at selected time intervals for (a) $\rho_{s}=910 \mathrm{kgm}^{-3}$ and (b) $\rho_{s}=6825 \mathrm{kgm}^{-3}$ at a strain rate $\dot{\varepsilon}=1.0 \times 10^{5}$ $s^{-1}$ with $t_{R}=9.3 \mu \mathrm{s}$. Degree of deformation in each cell is represented by a gray scale varying from 1 (no deformation) to 0 (full collapse). The cell size of the specimen given as $d_{a v}=25.2 \mu \mathrm{m}$.

\subsubsection{Effect of cell shape irregularities}

In this section the effect of cell shape irregularities over stress-strain response has been studied. In order to conduct a parametric study, different random cellular structures have been generated keeping the average cell size $d_{a v}=25.2 \mu \mathrm{m}$ constant. The randomness is characterized by the standard deviation $S_{d}$ of the cell size as described in Figure 2.9(b). In order to characterize the effect of randomness on the stress-strain response and associated deformation, a wide variety of cellular specimens from perfect honeycomb to highly irregular cellular specimens has been generated. The standard deviation $\left(S_{d}\right)$ in cell size $d_{a v}$ is varied over a wide range from $0 \mu m$ 
to $3.0 \mu \mathrm{m}$. All the specimens are fastened against a rigid support and are subjected to an impact velocity $v_{a}=50 \mathrm{~ms}^{-1}$ with a pulse rise time $t_{R}=9.3 \mu \mathrm{s}$ resulting in a strain rate of $\dot{\varepsilon}=1.0 \times 10^{5} \mathrm{~s}^{-1}$. Figure 4.17 illustrates the stress-strain responses of

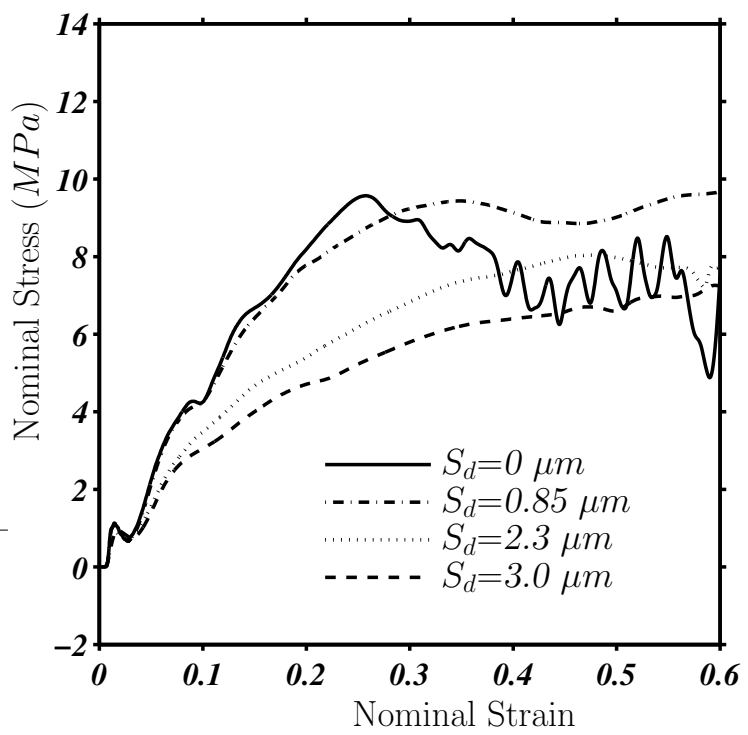

Figure 4.17: Stress-strain response of a cellular material for different randomness at strain rate $\dot{\varepsilon}=1.0 \times 10^{5} \mathrm{~s}^{-1}$ and for pulse rise time $t_{R}=9.3 \mu \mathrm{s}$. The cell size of the specimen is $d_{a v}=25.2 \mu \mathrm{m}$.

the cellular specimens with different randomness in the cell shape. It can be clearly seen that as the irregularities in the cell shape increases (i.e. $S_{d}=0.85$ to $3.0 \mu \mathrm{m}$ ), the plateau stress gets reduced appreciably. The effect of randomness on plateau stress has been studied numerically by Li et al. (2007) who also observed a similar trend. Interestingly, perfect honeycomb structure $\left(S_{d}=0\right)$ shows a different stressstrain response with a highly stiffening behavior upto a strain $\varepsilon=0.25$ followed by a softening behavior. The stress value after the softening period becomes almost comparable to the highly irregular cell $S_{d}=3.0 \mu \mathrm{m}$. The above responses of different irregular specimens will be presented with the help of deformed snapshots in the subsequent discussion.

Figure 4.18 shows the deformation pattern of cellular specimens with different cell irregularities. Three different specimens such as a perfect honeycomb $\left(S_{d}=0\right)$, a mod- 


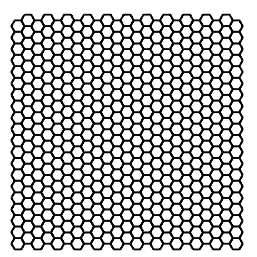

$t=0$

$\varepsilon=0$

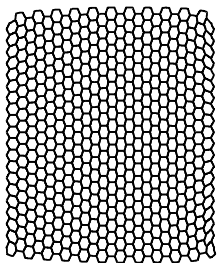

$t=4.3 \mu \mathrm{s}$

$\varepsilon=0.1$
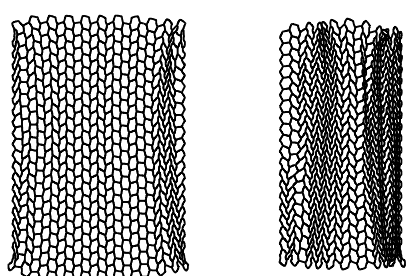

$t=6.7 \mu \mathrm{s}$

$\varepsilon=0.25$ $t=9.2 \mu \mathrm{s}$

$\varepsilon=0.47$

(a)
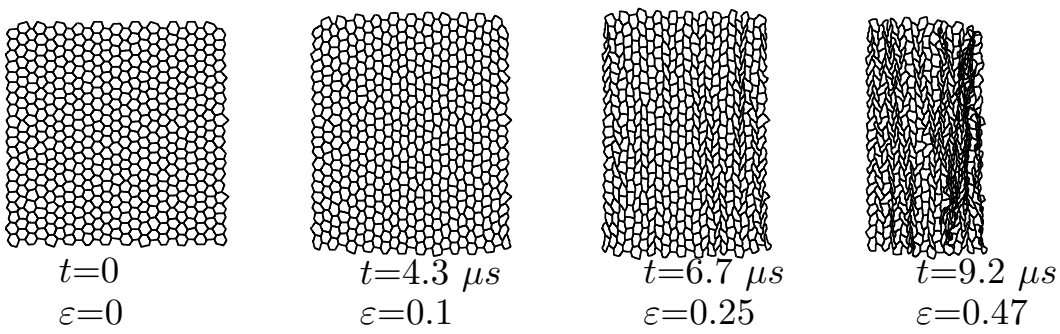

(b)
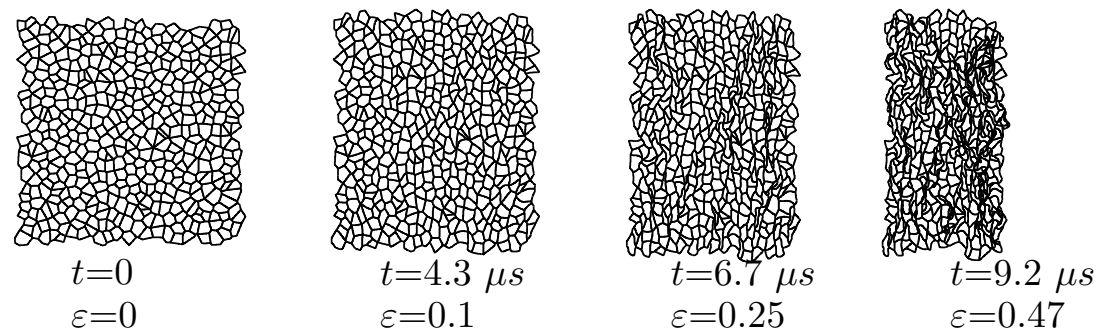

(c)

Figure 4.18: Snapshots of deformed specimens at selected time intervals for (a) $S_{d}=0$, (b) $S_{d}=0.85 \mu \mathrm{m}$ and (b) $S_{d}=3.0 \mu \mathrm{m}$ at a strain rate $\dot{\varepsilon}=1.0 \times 10^{5}$ $s^{-1}$ with $t_{R}=9.3 \mu s$.

erate irregular cellular specimen $\left(S_{d}=0.85 \mu \mathrm{m}\right)$ and a highly randomized specimen $\left(S_{d}=3.0 \mu \mathrm{m}\right)$ have been considered here. All the specimens show almost uniform deformation at $t=4.3 \mu \mathrm{s}(\varepsilon=0.1)$ and no presence of a collapsed zone at the impact end. Effect of microinertia could be expected to be almost similar for all the cases 
considered above and is also manifested by similar stress-strain responses at very early stage of deformation $(\varepsilon=0.05)$ in Figure 4.17. At $t=6.7 \mu s$, a larger number of collapsed cells throughout the domain can be found for the highly irregular cell shape specimen $\left(S_{d}=3.0 \mu \mathrm{m}\right)$. For other two cases, deformed cells are arranged in well defined bands. Due to the presence of weak sites throughout the domain for the most irregular case $\left(S_{d}=3.0 \mu \mathrm{m}\right)$, localization zones initiate early and randomly. Such early initiation of localization allows the irregular cell size specimen to crush at a lower stress level as found in stress-strain response. These weak sites in a sample with increasing randomness can be attributed to the initial misalignment of struts with respect to the loading direction. With increased randomness, the struts tend to possess increased initial inclination with respect to the loading direction thus making bending mode of deformation preferable. While buckling strength varies with the inverse of the square of the strut length, bending stiffness can be equated to the inverse of the third power of length. Thus rotation of struts commences at a lower load when bending mode of deformation is also present. Accordingly, cells collapse earlier in this scenario reducing the plateau stress of the cellular specimen. Perfect honeycomb as well as cellular specimens with moderate randomness show almost similar deformation pattern at $t=6.7 \mu \mathrm{s}$ and hence the stress-stress responses are quite similar upto a strain $\varepsilon=0.25$. For a perfect honeycomb and a moderate irregular specimen, most of the struts are almost aligned with the impact direction and hence exhibit a microbuckling dominated deformation mode resulting in a delayed collapse. At $t=9.2$ $\mu s$, the specimen with higher randomness continues to collapse at almost constant level of stress. At this stage, two different interesting deformation signatures can be found for perfect honeycomb and the cellular specimen with moderate randomness. The specimen with lower randomness shows a microbuckling as well as microbending dominated deformation zones distributed throughout the domain. In case of perfect honeycomb structure, layers of localized 'I' bands develop which reduces its strength appreciably. Thus it shows a lower plateau stress compared to irregular cellular spec- 
imen in Figure 4.18.

\subsubsection{Effect of cell orientation}

In this section we investigate the effect of cell orientation with respect to loading direction on the macroscopic behavior of cellular materials. Similar to previous analysis, two cellular specimens; a perfect honeycomb and highly irregular foam are subjected to impact velocity $v_{a}=50 \mathrm{~ms}^{-1}$ with a pulse rise time $t_{R}=9.3 \mu \mathrm{s}$ from the left and from the top. It can be observed from Figure 4.19 that perfect honeycomb offers much stiffer response when loaded from left direction than top. It is also observed that per-

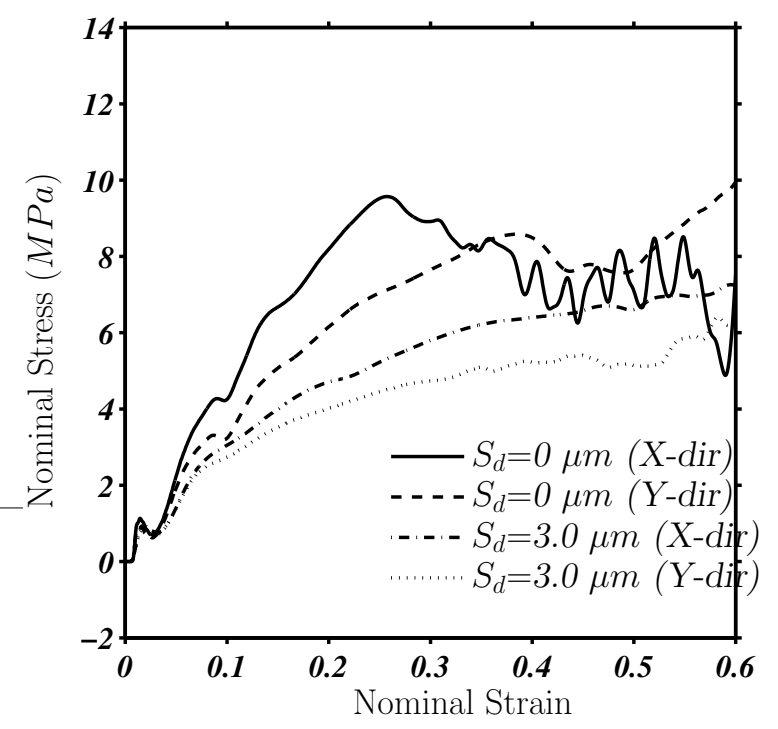

Figure 4.19: Stress strain response of cellular specimens with different cell irregularity subjected to loading in different directions.

fect honeycomb exhibits higher collapse strength when loaded from left. The irregular cellular structure shows almost similar stress-strain signatures when impacted from left as well as top.

To correlate the origin of such macroscopic response with the deformation behavior 
of cellular specimen, deformed snapshots of cellular specimens being impacted from top is depicted in Figure 4.20(a). It can be seen that perfect honeycomb when loaded from top, offers no deformation localization up to strain $\varepsilon=25 \%$. Hence, the stressstrain response exhibits no collapse. The applied deformation is accommodated by the cellular structure only due to microbending of the struts. No microbuckling occurs at this stage. Therefore, ' $\mathrm{X}$ ' type of deformation band emerges throughout the cellular domain. Therefore, the cellular structure offers a smooth response. On the other hand, for a perfect honeycomb the struts are aligned to the impact direction. It offers stiffer response as the axial stiffness dominates than the bending stiffness when loaded from top. However, it shows early collapse due to microbuckling of struts (presence of ' $\mathrm{I}$ ' bands). At higher strain (>25\%), perfect honeycomb exhibits an oscillatory

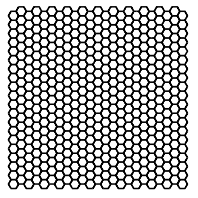

$\varepsilon=0$

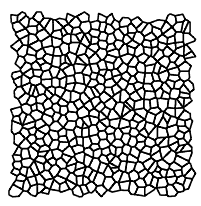

$\varepsilon=0$

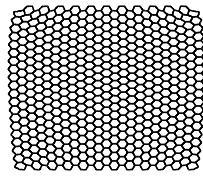

$\varepsilon=0.1$

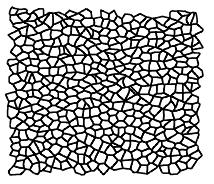

$\varepsilon=0.1$

(a)

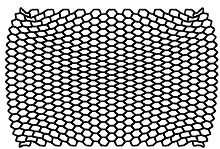

$\varepsilon=0.25$

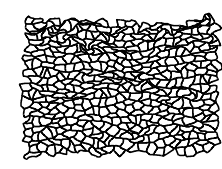

$\varepsilon=0.25$

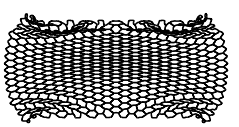

$\varepsilon=0.47$

(b)

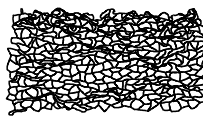

$\varepsilon=0.47$

Figure 4.20: Snapshots of deformed specimens when impacted from the top for (a) perfect honeycomb $S_{d}=0.0$ and (b) highly random microstructure $S_{d}=0.3 \mu \mathrm{m}$ at a strain rate $\dot{\varepsilon}=1.0 \times 10^{5} \mathrm{~s}^{-1}$ with $t_{R}=9.3 \mu \mathrm{s}$.

plateau response as it evolves multiple collapse band in the domain. The random cellular structure shows almost similar deformation signatures when loaded from left as well as top (Figure 4.20(b)). The difference in the magnitude of plateau stress as 
observed in Figure 4.19 can be originated from the emergence of distributed collapse zones due presence of microstructural variations.

\subsection{Concluding remarks}

In this study, we delineate the regimes of influences of microinertia and microbuckling on the high strain rate deformation behavior of cellular specimen. We computationally generated the initial stress enhancement and subsequent plateau region in the constitutive response, and studied the micromechanisms involved in it. Also, through a parametric study, we altered the stress enhancement, and subsequent plateau stress thus predicting the role of each micromechanism. We precisely observed different modes of cell collapse namely progressive and uniform, and discussed geometric, material and loading parameters that are responsible for the macro-observation. Our numerical simulations can predict experimental observation in several situations, and provides further insight into the problem. As we can map the deformation and stress state of the entire sample, we can relate these mechanical states with the overall deformation behavior and stress-strain curve. Thus, our computational framework gives a way to study the dynamic phenomena occurring within the specimen, and corresponding evolution of deformation and stress-strain response. Some of the salient findings are listed below.

1. Microinertia of the struts and the associated increase in the dynamic buckling strength is responsible for early stress enhancement, and is typically followed by the microbuckling and associated collapse of a few layers of cells at a time. This microbuckling event manifests itself by a rapid drop in the macroscopic stress strain response. In the absence of inertial enhancement, microscopic deformation is chiefly dominated by bending of cell walls at an angle to the loading 
axis, and is distributed uniformly throughout the domain. This phenomenon can be followed by microbuckling if the critical buckling stress for the struts is also achieved later in the loading history.

2. At higher strain rates, short pulse rise time leads to higher microinertial enhancement resulting in higher dynamic microbuckling strength. Consequently, the cells collapse at the impact end with varying degree of intensity depending on the rise time. However, the deformation patterns for all rise times considered here become similar after the initial transients and hence exhibit almost equal magnitude of plateau stress.

3. The effect of microinertia is less and almost similar for different cell sizes, and consequently deformation response is similar at the early stage. In the later stage of deformation, a larger cell size cellular specimen shows more localized deformation at the impact end compared to a smaller cell size specimen. This result is due to the lower buckling strength of the struts in the larger cell size specimen. Our observations reveal that plateau stress varies inversely with cell area (square of the cell size), in contrast to the earlier observations of inverse cell size dependence. The strain rate affects the plateau stress considerably at higher foam density (or lower cell size).

4. Variation in Young's modulus does not produce any significant microinertial enhancement as to be expected. However, a low value of Young's modulus results in a lower critical microbuckling load, and consecutively a progressive collapse mode is favored for lower Young's modulus specimens. A higher Young's modulus cellular material undergoes a rather homogeneous deformation throughout the specimen. The plateau stress varies linearly with Young's modulus of the specimen. The effect of strain rate is apparent only at very high values of it. Plateau stress does not show any sensitivity to it at low strain rate regimes. 
5. Bulk density of constituent material significantly alters the microinertial effect and resulting initial stress enhancement. Also, increased microbuckling phenomenon is apparent with higher density resulting in highly collapsed zone at the impact end.

6. Cell irregularity offers lower microinertial effect and hence no stress enhancement at early stages of deformation. Specimen with higher cell irregularities exhibits lower plateau stress due to early evolution of random collapsed zones in the entire specimen. A perfect honeycomb structure gives higher stress at the early stage of deformation; later it shows a lower plateau stress due to the initiation and immediate collapse of 'I' bands.

7. Cell alignment to the direction of impact significantly alters the stress-strain responses. The perfect honeycomb shows ' $\mathrm{X}$ ' type deformation band when loaded from top and offers higher collapse strength. The deformation pattern for random cellular specimen is almost independent of loading directions. The random structure exhibits similar macroscopic stress-strain response when loaded from left and top. 


\section{Chapter 5}

\section{Role of heterogeneity on the mechanical performance of byssus}

thread

\subsection{Introduction}

In this chapter we have undertaken a rigorous analysis of the composition-property relationship of the mussel byssus thread. The present study focuses on computationally obtaining maximum toughness of byssus thread and compares those against the experimentally reported values for different species. Using a Genetic Algorithm (GA) based combinatorial optimization technique; we evaluate the optimal percentage of proximal thread length for maximum toughness. To evaluate the toughness of a single thread, a mechanistic representation of byssus thread is developed that can capture the characteristic deformation features of a mussel byssus thread accurately. Moreover, using this optimization formulation, we categorize the contributing nondi- 
mensional parameters towards the optimal percentage of proximal thread length. Furthermore, by extracting those significant nondimensional parameters for different species, the optimal percentage of proximal thread length and associated maximum toughness are evaluated, and are compared with experimentally observed values. Our results substantiate that mussel threads arrange their chemical constitution to optimize a specific goal: achieve maximum toughness under tensile displacement. Such an extensive analysis of byssus thread may prove to be an ideal model for biomimetic design of tough threads.

This chapter is organized as follows: the detail of mathematical framework is presented in Section 5.2. In Section 5.3, a thorough discussion of effect of structural heterogeneity on the toughness characteristics is carried out. Of special interest is the effect of length percentage of proximal regions on the maximum toughness characteristics of different species. Finally, we end our discussion by drawing conclusions from the present study in Section 5.4.

\subsection{Numerical framework}

\subsubsection{Mathematical model}

A modeling framework is developed in this section to characterize the optimal functionality of byssus thread in terms of its energy absorption capability (toughness) along with specific requirements of strength and extensibility. Based on the experimental observation, a mechanical model is developed which will be robust enough to mimic the characteristic deformation features of byssus thread. To estimate the maximum toughness, an optimization formulation is incorporated which can explicitly account for all the material and geometrical properties through the mechanistic 


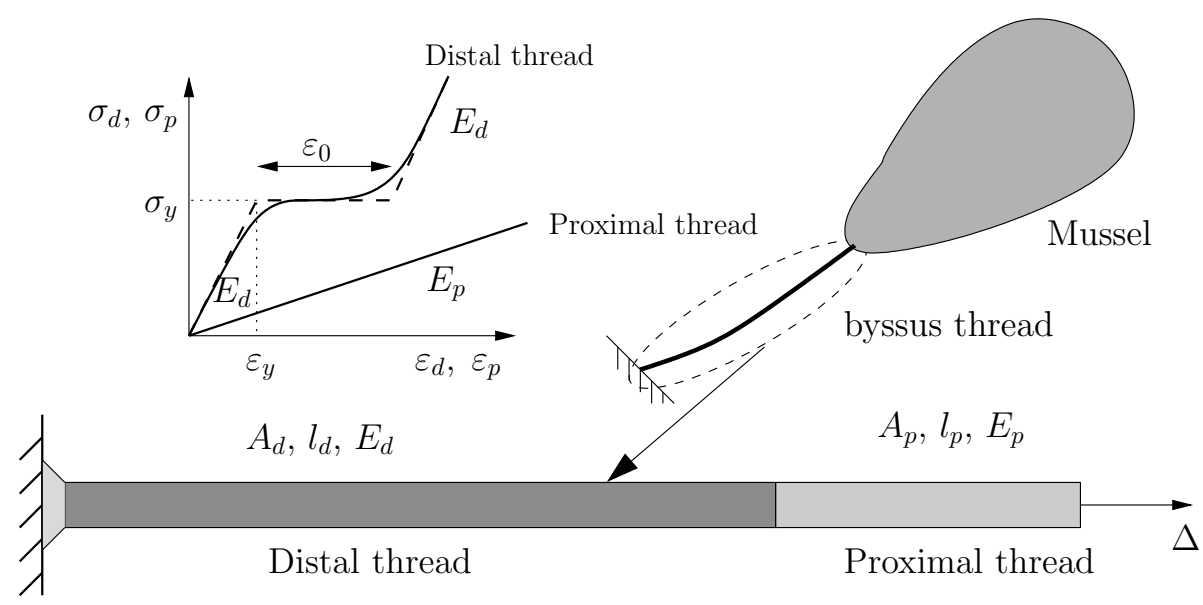

Adhesive plaque

Figure 5.1: Schematic representation of byssus thread showing different regions (proximal thread, distal thread and adhesive plaque). The geometric and material properties of these regions are shown in the figure. The constitutive laws of distal and proximal threads have been illustrated in the top figure. Dashed line in the constitutive law represents the idealized response of the distal portion.

representation of byssus thread. A detail description of mechanical model of the byssus is presented here. Figure 5.1 depicts a fully mechanistic representation of a typical byssus showing proximal and distal regions along with the adhesive plaque. Lengths of the distal and proximal portions of the thread are denoted by $l_{d}$ and $l_{p}$, respectively, whereas $L=l_{p}+l_{d}$, denotes the total length of the thread. We assume that the thread is of uniform cross-section. In the present study, we specifically interested to the species whose distal region shows a tri-phasic stress-strain response while proximal region offers approximately an elastomeric response. Correspondingly, an idealized constitutive material law represented by dashed line in Figure 5.1 has been assumed for the distal region. In the constitutive response, initially, the stress varies linearly with strain followed by a yielding at a constant stress, and finally it regains its initial stiffness by the virtue of its self-healing capability. $E_{d}, \varepsilon_{0}$ and $\sigma_{y}$ represent the Young's modulus, amount of plastic strain and yield stress, respectively. On the other hand, a purely linear elastic constitutive response is assumed for proximal region. The Young's modulus of the proximal region is represented by $E_{p}$. As most of 
the experimental observations show that the plaque is the critical zone of the overall thread failure consequently, in the present study we assume failure to be restricted in the adhesive plaque. Henceforth, we model plaque as a rigid-plastic point element with a failure strength $\sigma_{\text {pla }}$. Note that, a complete tri-phasic response is possible if plaque stress is higher than the yield stress of the distal thread $\left(\sigma_{p l a}>\sigma_{y}\right)$ and the distal thread must undergo the self-healing phase $\left(\varepsilon_{d}>\varepsilon_{y}+\varepsilon_{0}\right)$. The overall mechanical response of the composite thread when subjected to an applied displacement can be found by solving the following boundary value problem (BVP). The govering equations are given as

$$
\begin{aligned}
\nabla \boldsymbol{\sigma} & =\mathbf{0} \\
\boldsymbol{\varepsilon} & =\frac{1}{2}\left(\nabla u+\nabla u^{T}\right), \\
\boldsymbol{\sigma} & =\mathbb{C}: \boldsymbol{\varepsilon},
\end{aligned}
$$

subjected to boundary condition

$$
\mathbf{u}=\mathbf{u}_{0}
$$

The constitutive relations for proximal and distal regions are given as

$$
\begin{aligned}
& \sigma_{p}= E_{p} \varepsilon_{p}, \\
& \sigma_{d}= \begin{cases}E_{d} \varepsilon_{d}, & \text { if } \varepsilon_{d} \leq \varepsilon_{y}, \\
\sigma_{y}, & \text { if } \varepsilon_{y} \leq \varepsilon_{d}<\varepsilon_{y}+\varepsilon_{0}, \\
\sigma_{y}+E_{d}\left(\varepsilon_{d}-\varepsilon_{0}-\varepsilon_{y}\right), & \text { if } \varepsilon_{d} \geq \varepsilon_{y}+\varepsilon_{0} .\end{cases}
\end{aligned}
$$

where, $\varepsilon_{p}$ and $\varepsilon_{d}$ are strains in proximal and distal region, respectively. The plastic deformation of the distal region is described by the yield function as

$$
f:=|\sigma|-\sigma_{y}=0 .
$$


The above BVP is solved using the finite element scheme as developed earlier in Chapter 2. The force extension response is evaluate for byssus thread for different mussel species. Later, the finite element framework will be incorporated in the optimization formulation to evaluate the maximum energy absorption characteristic of the byssus thread under tensile displacement.

\subsubsection{Experimental validation}

To validate the present modeling framework, the deformation of a whole thread is evaluated for a mussel species $M$. californianus and is compared with experimentally observed response reported in literature. The geometric and material properties of this thread have been taken from Bell and Gosline (1996) and are represented in the form of meants.e.m. The length of thread is taken as $L=22 \mathrm{~mm}$ where length of the distal region $l_{d}=17 \mathrm{~mm}$, and length of the proximal region $l_{p}=5 \mathrm{~mm}$. The characteristic area of the thread is given as $a_{t}=5.89 \times 10^{-8} \pm 0.68 \times 10^{-8} \mathrm{~m}^{2}$. Young's modulus $E_{d}=868.6 \pm 181.2 \mathrm{MPa}$, yield stress $\sigma_{y}=15 \pm 1.0 \mathrm{MPa}$, and amount of plastic strain of distal thread before its self-healing $\varepsilon_{0}=0.4$. Young's modulus of proximal thread is given as $E_{p}=15.6 \pm 1.3 M P a$. The failure stress of the plaque is given as $\sigma_{f p l a}=32.25 \pm 3.9 \mathrm{MPa}$. It can be noticed that the experimentally observed material properties involve wide statistical variation about its mean value. Such a large variation of properties among threads appears due to condition of mussel health, thread age, equipment limitations and precise separation of threads into distal and proximal portions. Considering these material and geometric properties along with their variations, the force-displacement relationship is simulated and is presented as the shaded region in Figure 5.2(a). The experimentally observed response during tensile displacement is also illustrated for comparison purpose. A wide shaded region representing the extent of model response arises due to presence of large variations in material and 
geometric properties of byssus thread. It can be seen that the experimental observation lies within the range as predicted by the model. The model response clearly depicts the three significant phases of deformation of the byssus thread under tensile displacement. To explain deformation characteristic in different phases, we also find

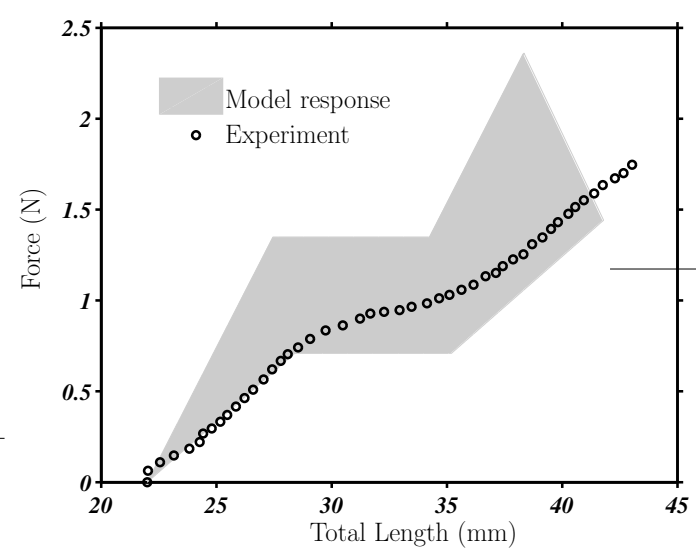

(a)

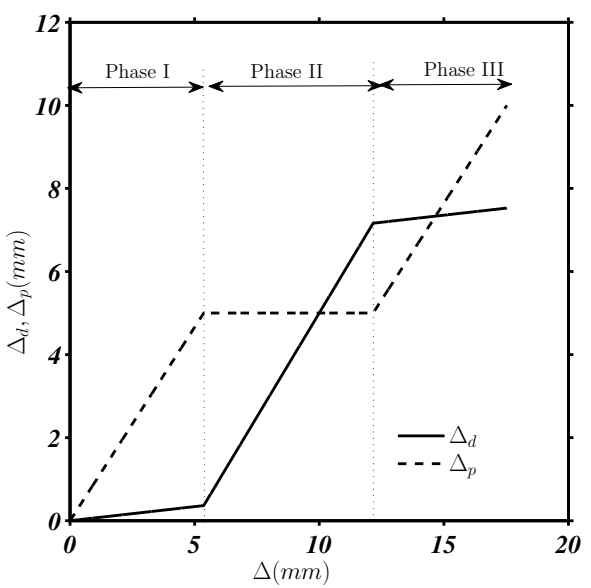

(b)

Figure 5.2: (a) Force vs total thread length $(L+\Delta)$ of a mussel byssus thread of species $M$. californianus. Shaded region represents the extent of tri-phasic response predicted by the model. (b) Individual extension of the proximal $\left(\Delta_{p}\right)$ and distal $\left(\Delta_{d}\right)$ regions due to applied tensile displacement $\Delta$.

the individual extension of distal $\left(\Delta_{d}\right)$ and proximal $\left(\Delta_{p}\right)$ regions due to applied tensile displacement $\Delta$. Initially, the force-extension response shows a stiffening behavior where proximal region is extended more (Figure 5.2(b)) compared to the distal region due less resistance offered by it. It the next phase, the distal region is extended due to yielding which is reflected through no increase in force in the force-extension behavior. Finally, combined deformation of distal and proximal regions contributes the last phase of the force-extension behavior. Similar to the initial phase, the proximal region undergoes a large deformation than the distal region. 


\subsubsection{Optimization formulation}

The objective of the current study is to identify the governing principle behind the heterogeneous distribution of the proximal and distal components of the byssus thread. Since the purpose of the byssus thread is to provide anchorage to the mussel, we hypothesize that primary design principle is in maximizing toughness of the thread. To test this hypothesis we have formulated an optimization formulation with the objective of determining the optimal proximal and distal length required in maximizing toughness. The optimization formulation can be written as

$$
\begin{gathered}
\text { Maximize } \quad U=\int_{\varepsilon} \sigma d \varepsilon, \\
\text { Subjected to: } \quad 0 \leq \xi \leq 1 .
\end{gathered}
$$

The objective in the above formulation is to maximize toughness, represented as $\int \sigma d \varepsilon$ and interpreted as the area under the stress-strain plot. The design variable which needs to be optimized is the distribution of the proximal and the distal portion of the byssal thread of total length $L$, represented in dimensionless form as $\xi=l_{p} / L$. The intricate nature of constitutive response of the whole thread offers a complex variation objective function $\int \sigma d \varepsilon$ with respect to the optimization variable $\xi$. Thus, evaluation of derivative of objective function is not amenable. Hence we adopted a combinatorial optimization technique, the Genetic Algorithm (GA), in solving the optimization formulation. Primary advantage of this technique is it relies on function evaluation only and does not need derivative evaluation.

GA (see Appendix E.2) represents a class of search and optimization procedures that are patterned after the biological process of natural selection and are extensively used 
in the solution of wide range of optimization problems. While solving an optimization problem using GA, each optimization variable is typically encoded as string of bits, and all strings are appended together to form a chromosome. Each individual in a population has a particular chromosome value that can be decoded to evaluate the parameter values and objective function, also called the fitness function. Populations are evolved through several generations until the objective function cannot be improved any further. The current formulation has a single optimization variable, $\xi$, which is represented by 10 bits, which constitutes the chromosome.

Unlike classical search and optimization methods, GA begins its search with a random set of solutions instead of just one solution. The detail steps of implementation of optimization problem are illustrated in Figure 5.3. The first step of GA procedure

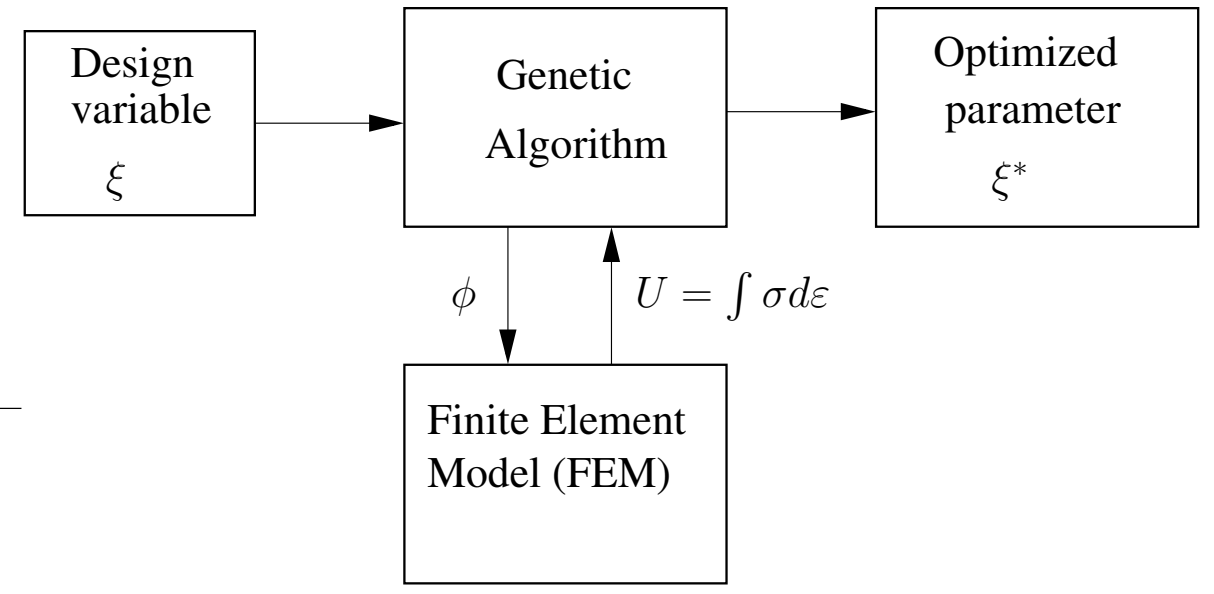

Figure 5.3: Steps of optimization formulation. Objective function of GA is evaluated through the finite element simulation.

(see Appendix E.2) thus involves the random generation of a population of binary strings, where each member of the population represents a value of $\xi(0 \leq \xi \leq 1)$. For each value of $\xi$ the fitness function is evaluated by performing the FEM simulation. The FEM simulation calculates the toughness for a given set of system parameters $\phi=\left\{\xi, E_{d}, E_{p}, \sigma_{y}, \varepsilon_{0}, \varepsilon_{\max }, \sigma_{p l a}\right\}$, which contains the design variable and other fixed variables. The generated population of solutions is modified by the GA operators 
(reproduction, crossover, mutation) to create new and better populations. This procedure is repeated until a pre-determined termination criterion is satisfied. While the primary advantage of using GA in this problem is to avoid gradient evaluation, it also poses better chance of determining the global optima. The above formulation of the non-linear programming problem is complex in nature and hence is likely to be non-convex with multiple local minima. Application of classical gradient-based solution techniques for such a problem will be strongly dependent on the initial guess of the starting solution, which has to be modified to move towards the global solution. However, even in classical techniques there will be no guarantee of global solution for the non-convex problem. Although guarantee of global solution cannot be provided in GA as well, it has the advantage of working with multiple starting points or populations, which are simultaneously converged, thereby eliminating possible local minimas in the process. On the other hand, however, the optimal solution obtained by GA depends heavily on the termination criteria predetermined by the user, which can be a limitation. In the present study, the simulation was allowed to run sufficient number of times until no significant change in the objective function is observed. Details of the parameters used for current simulation is elaborated in Table 5.1.

\begin{tabular}{lll}
\hline \hline Parameter & Description & Value \\
\hline$N_{\max }$ & Maximum number of generations & 50 \\
$N_{\text {pop }}$ & Number of population & 10 \\
$n b i t s$ & Number of bits & 10 \\
$p_{c}$ & Crossover probability & 0.5 \\
$p_{m}$ & Mutation probability & 0.02 \\
\hline
\end{tabular}

Table 5.1: List of different GA parameters.

\subsection{Result and discussions}

Detailed analyses are performed in this section to test our hypothesis that there exists a particular percentage of proximal length in byssus thread which enables it to offer 
the maximum energy absorption capability under applied tensile displacement. As found in literature, the plaque strength $\left(\sigma_{p l a}\right)$ plays a crucial role for the survivability of mussel under the wave swept environment. Moreover, it is apparent that higher plaque strength is always preferable to ensure its attachment. On the other hand, extensibility offered by the byssus thread under a tensile stretching is also a major parameter towards the overall mechanical performance of mussel in a wave swept environment. As the different amount of slackness can be assumed to be involved with each byssus thread to a particular load direction arising from breaking water waves, higher extensibility allows other byssus threads (Bell and Gosline, 1996) to align in the loading direction for redistributing the load among them. Hence, through this mechanism thread reduces the probability of the attainment of failure strength $\sigma_{f}\left(=\sigma_{p l a}\right)$ and thus ensures its attachment. So, it can be inferred that both failure strength $\sigma_{f}$ and maximum extensibility, which is defined as maximum failure strain exhibited by the whole thread, $\varepsilon_{\max }$ are the main constraints of the present scheme for optimal performance.

Considering above constraints, the toughness $\left(U=\int \sigma d \varepsilon\right)$ of the byssus thread of $M$. californianus is obtained for different proximal lengths. Using similar material and geometric parameters as described in the previous section, percentage of the proximal length $\xi$ is varied from 0 to 1 . The thread becomes a completely distal thread when $\xi=0$, whereas a fully proximal thread is described by $\xi=1$. Consequently, $0<\xi<1$ indicates a thread composed of both proximal and distal regions. The maximum extensibility of the whole thread is considered as $\varepsilon_{\max }=0.9$. Figure $5.4(\mathrm{a})$ shows that initially toughness increases as the proximal length increases and reaches its maximum at $\xi^{*}=l_{p}^{*} / L$. A further increase of $\xi$ rapidly reduces the toughness. To describe this fact in more detail, a map is constructed in Figure 5.4(b) that shows how the constraints are satisfied under tensile displacement for different values of $\xi$. The figure illustrates the normalized stress, normalized strain of the thread, and modes of deformation such as phase I (initial stiffening), phase II (yielding of distal 


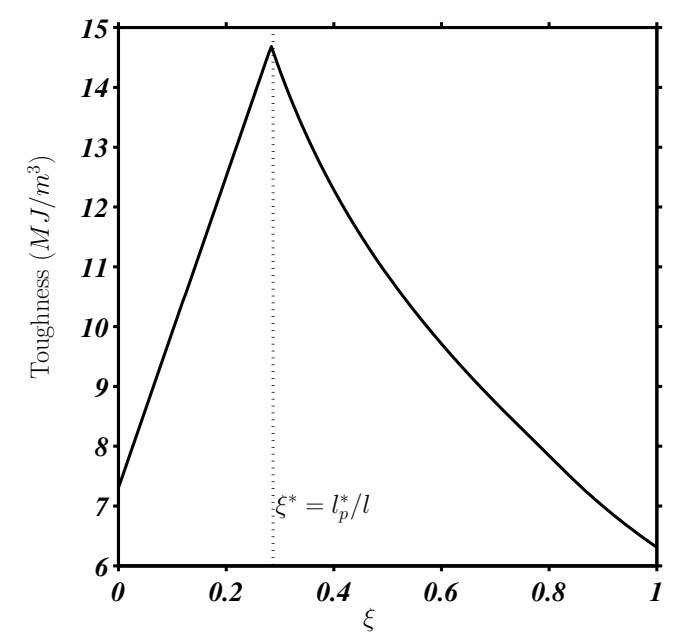

(a)

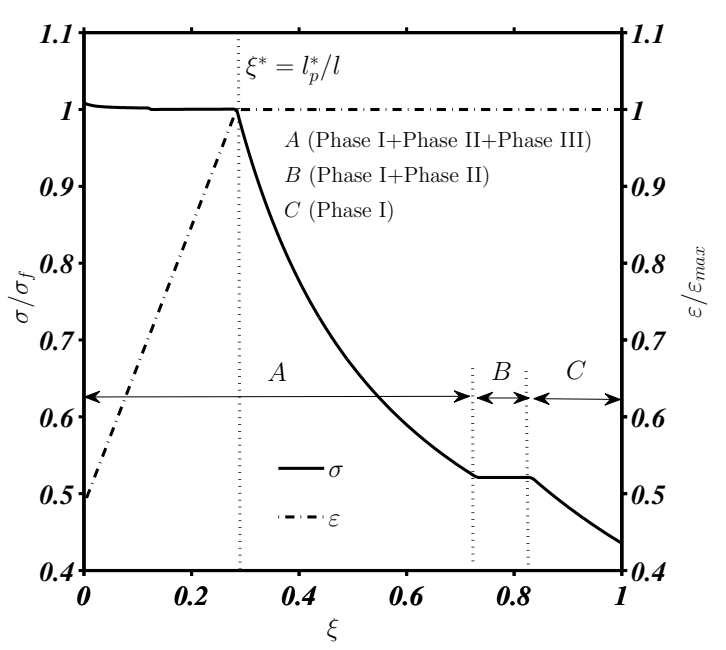

(b)

Figure 5.4: (a) Variation of toughness $(U)$ of the whole thread for different percentage of proximal thread length $\xi$. (b) A map presents the condition at which maximum stress $\left(\sigma_{f}\right)$ and maximum extensibility $\left(\varepsilon_{\max }\right)$ criteria are achieved for different percentage of proximal thread length $\xi$. Types of overall deformation behavior such as tri-phasic $(A)$, bi-phasic $(B)$ and mono-phasic $(C)$ responses of the whole thread for different values of $\xi$ are also indicated.

thread) and phase III (self-healing of distal thread), it has undergone. For $\xi<\xi^{*}$, the stress reaches to the plaque strength (maximum stress) and the thread separates from the surface. Hence, it allows byssus thread to absorb less amount of energy prior 
to detachment. It can also be noticed from Figure 5.4(b) that the composite thread undergoes a complete tri-phasic deformation (phase I, phase II, and phase III). In case of $\xi>\xi^{*}$, thread offers maximum extensibility without reaching the plaque strength. Thus, it engages other threads to sustain the load and hence confirms its survivability. As proximal length increases the energy absorption at phase II and phase III reduces which decreases the overall toughness. As the contribution of distal portion reduces with higher $\xi$, according to Figure $5.4(\mathrm{~b})$ at higher values of $\xi \approx 0.725$, thread response switches from a tri-phasic response to a bi-phasic response (phase I and phase II). As the distal thread is not stretched enough to undergo its self-healing phase, a biphasic response of whole thread emerges. However, this bi-phasic response exists for a small interval of $0.725 \leq \xi<0.8$ and transforms to a completely mono-phasic response (phase I) for $\xi \geq 0.8$. A mono-phasic response appears as the distal thread offers no yielding. Thus, the deformation of distal thread controls the overall of mode of deformation. It can be noticed that, at $\xi=\xi^{*}$, the thread provides optimized strength as well as extensibility, and consequently offers the maximum toughness. Note that, the predicted $\xi^{*}=0.28$ for $M$. californianus is comparable to experimentally observed percentage of proximal length $(\xi=0.21 \pm 0.03)$. The above results clearly show the significance of $\xi$ in determining the material properties of the byssus thread including strength, extensibility and toughness. In order to investigate the contribution of $\xi$ in a structured way, we have formulated an optimization problem (Equation 5.6) which determines the optimal value of $\xi$ required to achieve maximum toughness. Hence, the percentage of proximal thread length $\xi=l_{p} / L$ is considered as the free variable in the optimization formulation as given in Equation (5.6). Using the optimization formulation as described in Section 5.2 , the optimal proximal length fraction $\xi^{*}$ will be determined for different species. However, different mussel species possess significant differences in material parameters (see Table 5.2). Thus before embarking upon determining the optimal design principle, we will first attempt to identify the critical inter-species parameters. 


\subsubsection{Identification of critical parameters}

As evident from the mathematical model, evaluation of stress-strain relationship requires numerous material parameters such as $E_{d}, \sigma_{y}, \varepsilon_{0}, E_{p}$ and $\sigma_{f}$. While these parameters are unique for species, they have significant inter-species variations. Hence, our first task will be to determine the effect of variations of these material properties on the optimal value of $\xi$ as determined from the solution of the optimization problem. The purpose of this analysis is to determine the sensitivity of the optimal solution to the individual material property, with the final aim of determining the most critical one. Note that, we have already assigned the percentage of proximal thread length $\xi$ as the most critical parameter to be evaluated and kept it as a free variable. Initially, we will construct a set of nondimensional parameters and will systematically examine the effect of those parameters on the optimal percentage of proximal thread length using the GA based optimization formulation. Therefore, a set of contributing nondimensional parameters will be identified which will enable designer to select the constituent materials easily for efficient biomimetic design of byssus thread.

First we will analyze the effect of the ratio of the Young's modulus of distal thread to proximal thread, defined as $\chi=E_{d} / E_{p}$, along with yield stress of distal thread $\sigma_{y}$ on the optimal solution $\xi$. To evaluate that, the optimization problem is solved to determine the evolution of $\xi^{*}$ over the entire range of range of $\chi$ from 5 to 75 as well as $\sigma_{y} / E_{p}$ from 0.5 to 1.5 . During these simulations the parameters which are kept fixed are: the plastic strain of the distal part $\varepsilon_{0}=0.4$, the maximum extensibility exhibited by the whole thread is assumed $\varepsilon_{\max }=0.9$, the ratio of maximum strength (plaque strength) to proximal modulus is taken as $\sigma_{f} / E_{p}=2.0$ since many of the reported species it is observed to be the range of 1.8-2.5. Figure 5.5 plots the variation of $\xi^{*}$ with $\chi$ at different values of $\sigma_{y} / E_{p}$. It is observed that $\sigma_{y} / E_{p}$ has no effect on the optimal solution of $\xi^{*}$. Moreover, Figure 5.5 shows that $\xi^{*}$ rapidly increases for 


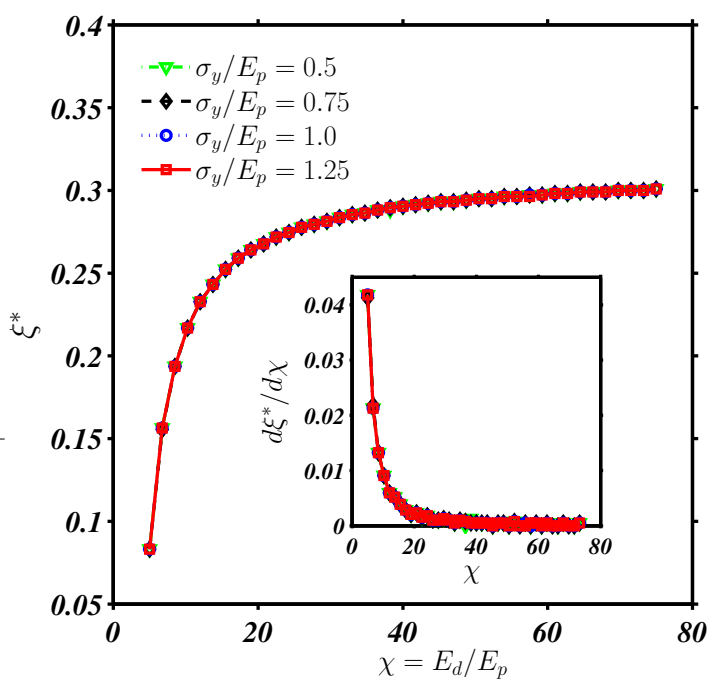

Figure 5.5: Variation of optimal percentage of proximal thread length $\xi^{*}$ for different ratios of Young's modulus of distal thread to proximal thread $(\chi)$ with a wide range of $\sigma_{y} / E_{p}$.

higher values of $\chi$ and does not show any considerable change when it reaches to a limiting value, defined as $\chi^{*}$. In order to estimate $\chi^{*}$, we plot $d \xi^{*} / d \chi$ for different values of $\xi$ in inset of Figure 5.5. It can be seen that at $\chi>40, d \xi^{*} / d \chi$ reduces to zero hence we assign $\chi^{*} \approx 40$. As toughness does not increase for $\chi^{*}>40$, it can be thought as design criterion for proximal and distal threads to fabricate tough composite thread with optimal material consumption. Interestingly, several species (Table 5.2) except $M$. californianus follow the similar requirement i.e., $\chi<\chi^{*}$. We think, a higher value of $\chi$ for $M$. californianus species is assumed to occur due to the presence of more rigid distal thread compared to the other species. Higher

\begin{tabular}{lllll}
\hline \hline Sepecies & $E_{d}(M P a)$ & $E_{p}(M P a)$ & $\sigma_{f}(M P a)$ & $\chi=E_{d} / E_{p}$ \\
\hline M. californianus $^{*}$ & $868.6 \pm 181.2$ & $15.6 \pm 1.3$ & $32.25 \pm 3.9$ & $55.6 \pm 14$ \\
M. edulis $^{\dagger}$ & $532 \pm 39$ & $77.5 \pm 11.6$ & $142.8 \pm 11$ & $6.9 \pm 1.1$ \\
M. galloprovincialis $^{\dagger}$ & $565 \pm 55$ & $50.5 \pm 3.8$ & $123.4 \pm 14$ & $11.2 \pm 1.3$ \\
\hline
\end{tabular}

Table 5.2: Material parameters for different mussel species. $\chi$ is evaluated considering the mean value of $E_{d}$ and $E_{p}$.

Young's modulus $E_{d}$ of $M$. californianus can be thought as a result of close packing

\footnotetext{
* Bell and Gosline (1996)

$\dagger$ Lucas et al. (2002)
} 
of its large number of load bearing segments (collagen fibers) in its ultrastructure. It essentially signifies further material (collagen molecule preCol-D) consumption in distal thread and correspondingly, associated fabrication cost is increased. However, no significant change in the optimal performance can be achieved with higher value of $\chi$. Thus, a higher $\chi$ can be thought as a consequence of over-designed thread without any appreciable gain in the target mechanical performance in terms of maximum toughness.

Interestingly, it can also be found from Figure 5.5 that the ratio of $\xi^{*}=l_{p}^{*} / L$ is independent of $\sigma_{y} / E_{p}$ which indicates that the yield stress of distal part does not affect the optimum proximal length criterion. For an optimal percentage of proximal thread length $\xi^{*}$, it can be seen that the thread can absorb significant amount of energy in the phase II with higher yield stress $\left(\sigma_{y}\right)$ of distal thread. Thus, a higher yield stress of the distal thread greatly influences the overall toughness of the byssus thread. The yield stress of distal thread mainly depends on the slippage or dislocation within the cross-linking or crystalline sites (Vaccaro and Waite, 2001; Harrington and Waite, 2007). Manipulating the resistance in slippage mechanisms of load bearing units in the ultrastructure of distal thread will lead to the development of thread with enhanced toughness. However, fabrication of this thread does not require any change to the optimal percentage of the proximal thread length.

Similarly, to find the effect of maximum failure strength (plaque strength) on the optimum performance of the byssus thread, the percentage of proximal length is determined for $\sigma_{f} / E_{p}$ along with $\chi$. Figure 5.6 represents the entire surface of the variation of $\xi$ with respect to $\sigma_{f} / E_{p}$ and $\chi$. Again, plastic strain of the distal thread $\left(\varepsilon_{0}\right)$ and maximum strain of the thread $\left(\varepsilon_{\max }\right)$ remain constant similar to previous analysis. The ratio of yield stress to Young's modulus of the proximal region is taken as $\sigma_{y} / E_{p}=0.75$. It can be observed that the percentage of proximal length $\xi^{*}$ greatly reduces with $\sigma_{f} / E_{p}$ for a fixed value of $\chi$. Analogous to the previous 


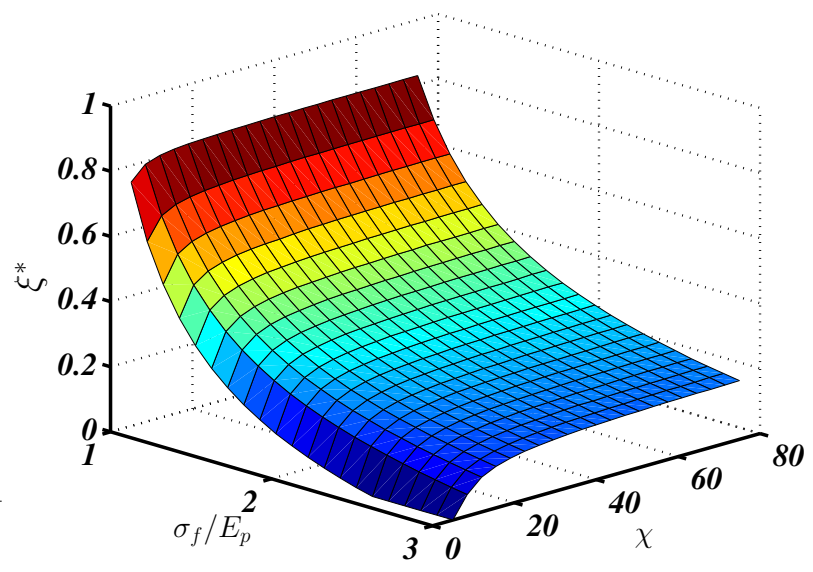

Figure 5.6: Optimal surface of percentage of proximal thread length $\xi$ for different $\chi$ and ratios of failure stress to Young's modulus of proximal thread $\sigma_{f} / E_{p}$.

analysis, the surface of $\xi^{*}$ shows a significant dependence on $\chi$ until, $\chi \approx \chi^{*}$. Thus, failure stress plays a crucial role to alter the percentage of proximal thread length to achieve maximum toughness. Therefore, the plaque strength can be considered as a contributing parameters which controls the optimal design of thread to achieve maximum toughness. Consequently, for biomimetic design of this thread, one needs to consider the maximum stress (plaque strength) as a critical design parameter. Note that the map of $\xi^{*}$ in Figure 5.6 may provide a design chart for development of a novel composite thread with maximum toughness characteristics combined with enhanced strength and extensibility. Henceforth, in the subsequent section, we will evaluate the effect of this parameters on the percentage of proximal thread length for different species.

In the present study, we assume that the optimum percentage of proximal thread length depends on the other nondimensional parameters through a complex functional form as

$$
\xi^{*}=\frac{l_{p}^{*}}{L}=f\left(\frac{\sigma_{f}}{E_{p}}, \chi, \frac{\sigma_{y}}{E_{p}}, \varepsilon_{0}, \varepsilon_{\max }\right) .
$$

As the previous analyses suggest that $\sigma_{y} / E_{p}$ ratio does not contribute towards the 
optimal percentage of proximal thread length, for a fixed plastic strain of distal thread $\left(\varepsilon_{0}\right)$ and maximum extensibility $\left(\varepsilon_{\max }\right)$ offered by thread, the above functional form can be further reduced to

$$
\xi^{*}=f\left(\frac{\sigma_{f}}{E_{p}}, \chi\right) .
$$

Finally, it can be concluded that the optimal percentage of proximal length ratio $\xi^{*}$ mainly depends on the two nondimensional parameters found as $\sigma_{f} / E_{p}$ and $\chi$. So, the a map of $\xi^{*}$ as found in Figure 5.6 for different $\sigma_{f} / E_{p}$ and $\chi$ will enable us to find $\xi^{*}$ for different species. In the following, we will determine the $\xi^{*}$ for different species and will compare with the experimentally reported values. As the experimentally reported byssus thread parameters involve large statistical variations, one should account the effect of this deviation on the estimation of optimal percentage of proximal length $\xi^{*}$ for achieving optimal performance.

\subsubsection{Evaluation of optimal percentage of proximal thread length}

To establish the hypothesis as stated earlier, we finally estimate the optimal percentage of proximal thread length $\xi^{*}$ for different species by extracting their nondimensional parameters. We have already investigated that the nondimensional parameter $\sigma_{y} / E_{p}$ does not contribute to the optimal percentage of proximal length $\xi^{*}$ however it alters the magnitude of the maximum toughness. Considering the variation of $\chi$ which arises due to presence of large scatter of $E_{d}$ and $E_{p}$ along with plastic strain of distal $\varepsilon_{0}=0.4 \pm 0.02$ as well as maximum extensibility $\varepsilon_{\max }=0.9 \pm 0.02$, the optimal percentage of proximal thread lengths $\xi^{*}$ are predicted in Figure 5.7 for a wide range $\sigma_{f} / E_{p}$ corresponding to mussel species $M$. galloprovincialis. The shaded region indicates the extent of $\xi^{*}$ attributed due to presence of statistical variation of $\varepsilon_{0}, \varepsilon_{\max }$ and $\chi$. Figure 5.7 shows $\xi^{*}$ reduces rapidly along with $\sigma_{f} / E_{p}$. The ex- 


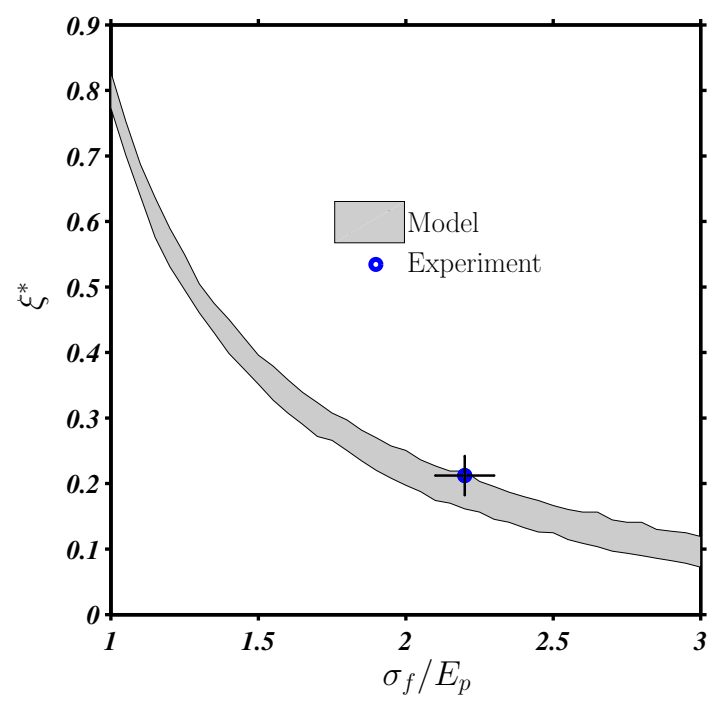

Figure 5.7: Variation of optimal percentage of proximal thread length $\xi^{*}$ with $\sigma_{f} / E_{p}$ for M. galloprovincialis. Shaded region represents the extent of optimal percentage of proximal thread length as predicted by the model. Experimentally observed percentage of proximal thread length is also indicated on the plot.

perimentally observed percentage of proximal thread length is also marked on the same figure. The horizontal error bar associated with the experimental data signifies the associated variation of $\sigma_{f}$ as well as $E_{p}$. It can be noticed that for the species M. galloprovincialis, the model prediction is close to experimentally observed value. We have already found that predicted percentage of proximal thread length for $M$. californianus is comparable to its experimental findings. Next, we will compare the percentage of proximal thread length and the maximum toughness of different species along with their experimental findings.

Figure 5.8(a) compares predicted optimal percentage of proximal thread length $\xi^{*}$ and toughness with experimentally observed values for different species. It can be noticed that the predicted optimal percentage of proximal thread lengths $\xi^{*}$ are within the limit of experimentally observed values. Such an agreement is supported by the overlapping error bars in predicted values and experimental findings. Similarly, we also compare the maximum toughness predicted by the present model with experi- 
mentally obtained toughness for different species in Figure 5.8(b). Interestingly, it

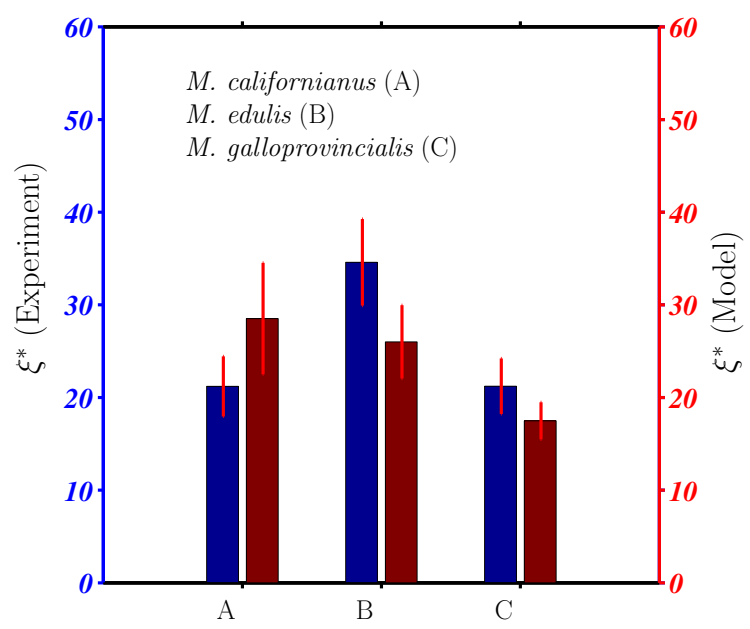

(a)

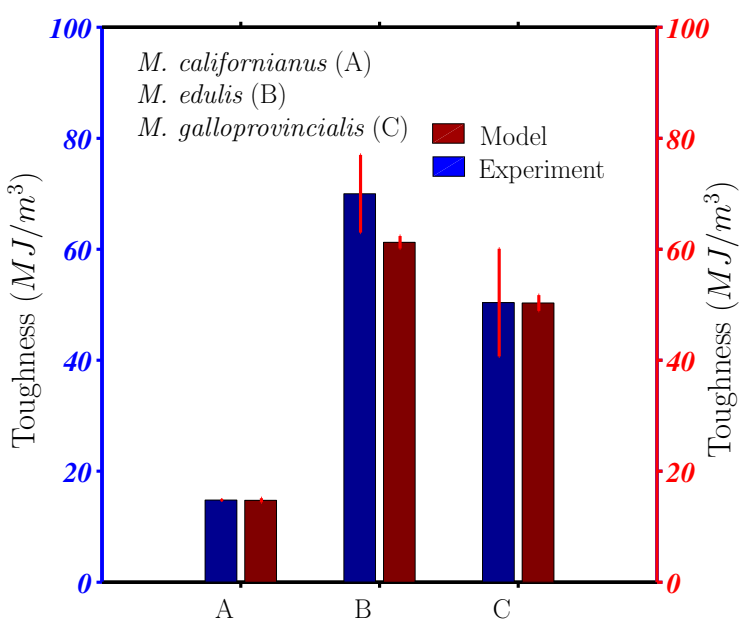

(b)

Figure 5.8: Comparison of (a) percentage of proximal thread length and (b) maximum toughness as predicted by the model with experimentally observed values for different mussel species. Simulations are performed taking into account experimental variations of model parameters resulting in variation in the simulation results denoted by error bars.

can be found that maximum toughness values are almost equal to the experimentally reported values. Hence, it can easily be inferred that toughness offered by a byssus thread during tensile stretching experiment is the maximum toughness for 
that thread. Furthermore, we have already investigated that the maximum toughness associated with different species originates due to the presence of a particular percentage of proximal thread lengths $\left(\xi^{*}\right)$ which are also comparable to experimentally observed values. Although, different material properties exist in the distal and proximal threads for different species, still they meet a common specific requirement, i.e., to achieve maximum toughness. Therefore, it can be inferred that collagen molecules mainly preCol-P and preCol-D are optimally arranged in the ultrastructure of the thread of different species to meet the above requirement. Moreover, this optimal arrangement is manifested at macroscale in terms of two different regions of thread such as proximal and distal threads. Therefore, the extraordinary toughness along with higher strength and extensibility of mussel byssus thread as seen in nature emerges from the presence of macroscale structural heterogeneity of byssus thread in terms of percentage of proximal thread length. Thus, the present findings confirm the hypothesis as stated earlier.

\subsection{Concluding remarks}

In this chapter, the origin of the high strength, toughness and extensibility of mussel byssus thread is investigated through an optimization framework along with mechanistic representation of thread. It is hypothesized that composite nature of the thread of all species aims to achieve maximum toughness which is an important criterion for their survivability in harsh environment. Using the proposed methodologies, the contributing nondimensional parameters are identified. Next, the percentage of proximal length and maximum toughness are predicted and compared with the experimental counterpart. The following conclusions can be drawn from the present analysis.

- Optimal percentage of proximal thread length highly depends on the ratio of 
failure strength to the Young's modulus of proximal thread and ratio of Young's modulus of distal thread to proximal thread. The higher value the ratio of Young's modulus of distal thread to proximal thread does not alter the optimal percentage of proximal thread length.

- Yield strength of the distal region has no impact on the optimal percentage proximal region. However, it makes the thread tougher.

- Different types of collagen (preCol-P and proCol-D) are optimally distributed along the thread which is manifested as stiff distal thread and soft proximal thread targeting to achieve high toughness along with high extensibility. 


\section{Chapter 6}

\section{Surrogate modeling techniques}

\section{applied to heterogeneous materials}

\subsection{Introduction}

Quite often in engineering computations we encounter the issue of computational cost. While advanced numerical techniques along with increased model resolution has enabled accurate mechanistic simulations, such advancement has introduced the burden of increased computational demand. This problem is commonly encountered across the borders of science and engineering: In the areas of design optimization, multi-scale modeling, turbulent combustion and fracture mechanics, to name a few. While details of the problem definition is specific to the system under study, the approach to alleviate the problem follows a similar principle across the discipline: replacing the detailed expensive model by a reduced order surrogate model. Two desired properties of these surrogate modeling techniques are that (1) they must be computationally efficient, and (2) they should represent the original model sufficiently well. Typical methods 
employed are proper orthogonal decomposition (POD), response surface method and look-up tables as discussed in Section 1.2.1.3. In the present study, we propose the Higher Dimensional Model Representation (HDMR) based surrogate model to reduce computational burden in several areas of computational material design such as construction of failure envelope of highly heterogeneous microstructure and multiscale analysis of nonlinear heterogeneous materials.

This chapter is organized as follows: the mathematical foundation of HDMR technique in given in Section 6.2. In Section 6.3, its potential application in the areas of failure envelope construction for heterogeneous materials is outlined. We propose surrogate model based multiscale framework for nonlinear heterogeneous materials in Section 6.4. Finally, we summarize our findings in Section 6.5.

\subsection{Mathematical foundation of HDMR}

Higher Dimensional Model Representation (HDMR) is an efficient approach to capture the high dimensional input-output (IO) behavior of a complex system. The fundamental principle behind HDMR representation is that the impact of multiple input variables on the output can be independent and cooperative. Let $f(\mathbf{Z})$ be a real valued function, which depends on the a $N$-dimensional random vector as $\mathbf{Z}=\left(Z_{1}, Z_{2}, \ldots, Z_{N}\right) \in \Re^{N}$. The HDMR of $f(\mathbf{Z})$ can be represented as a hierarchical correlated function expansion as

$$
\begin{aligned}
f(\mathbf{Z}) & =f_{0}+\sum_{i=1}^{n} f_{i}\left(Z_{i}\right)+\sum_{i_{1}<i_{2}}^{N} f_{i_{1} i_{2}}\left(Z_{i_{1}}, Z_{i_{2}}\right)+\sum_{i_{1}<i_{2}<i_{3}}^{N} f_{i_{1} i_{2} i_{3}}\left(Z_{i_{1}}, Z_{i_{2}}, Z_{i_{3}}\right) \\
& +\ldots+f_{12 \ldots N}\left(Z_{1}, Z_{2}, \ldots, Z_{N}\right) .
\end{aligned}
$$

In this expansion, 
- $f_{0}$ is the zeroth-order effect which is constant,

- $f_{i}\left(Z_{i}\right)$ represents the component function describing the independent action of variable $Z_{i}$ on the output,

- $f_{i_{1} i_{2}}\left(Z_{i_{1}}, Z_{i_{2}}\right)$ offers the co-operative effects of the variables $Z_{i_{1}}$ and $Z_{i_{2}}$ on the output,

- $f_{12 \ldots N}\left(Z_{1}, Z_{2}, \ldots, Z_{N}\right)$ gives any residual dependence on all variables in a cooperative way to influence the output.

While this expansion is an exact representation of the model output, HDMR is based on the fundamental principle that correlations between the independent variables affecting the system's output diminishes rapidly. Traditional statistical analysis of model behavior has revealed that only low order correlations can describe the process dynamics with sufficient accuracy. Based on this observation, second order HDMR expression should be sufficient in accurately representing high dimensional systems (Rabitz and Alis, 1999). The expression for second order HDMR takes the form:

$$
f(\mathbf{Z}) \approx f_{0}+\sum_{i=1}^{n} f_{i}\left(Z_{i}\right)+\sum_{i_{1}<i_{2}}^{N} f_{i_{1} i_{2}}\left(Z_{i_{1}}, Z_{i_{2}}\right)
$$

The HDMR component functions, $f_{0}, f_{i}\left(Z_{i}\right), f_{i_{1} i_{2}}\left(Z_{i_{1}}, Z_{i_{2}}\right), \ldots$ are found by minimizing the error functional as

$$
\int\left[f(\mathbf{Z})-f_{0}-\sum_{i=1}^{N} f_{i}\left(Z_{i}\right)-\sum_{i_{1}<\ldots<i_{s}} f\left(Z_{i_{1}}, Z_{i_{2}}, \ldots, Z_{i_{M}}\right)\right]^{2} d \rho(\mathbf{Z})
$$

under suitable specified orthogonality conditions which guarantee that all the component functions are determined step by step. Let us define $\mathcal{D}:=\{1,2, \ldots, N\}$ and let $\mathbf{u}:=\left\{i_{1}, i_{2}, \ldots, i_{M}\right\}$ denote the set of the coordinate indices, where $\mathbf{u} \subseteq \mathcal{D}$. The dimension of $\mathbf{u}$ is given as $M=\operatorname{dim}(\mathbf{u})$. The projection operator $\mathcal{P}: \Re^{N} \rightarrow \Re^{M}$ 
constructed as

$$
\mathcal{P}^{\mathbf{u}} \equiv \mathcal{P}^{i_{1} i_{2} \ldots i_{M}}:=\int_{\Re^{N-M}} f(\mathbf{Z}) d \rho_{\mathcal{D} / \mathbf{u}}(\mathbf{Z})
$$

where the measure $d \rho_{\mathcal{D} / \mathbf{u}}(\mathbf{Z})$ given as,

$$
d \rho_{\mathcal{D} / \mathbf{u}}(\mathbf{Z}):=\prod_{i \notin \mathbf{u}} d \rho\left(Z_{i}\right)
$$

The component functions of HDMR are evaluated through the application of suitably defined set linear operators $\mathcal{L}_{0}, \mathcal{L}_{i}, \mathcal{L}_{i_{1} i_{2}}, \ldots$ such that

$$
\begin{aligned}
f_{0} & \equiv \mathcal{L}_{0} f(\mathbf{Z})=\mathcal{P} f(\mathbf{Z}) \\
f_{i}\left(Z_{i}\right) & \equiv \mathcal{L}_{i} f(\mathbf{Z})=\mathcal{P}^{i} f(\mathbf{Z})-\mathcal{L}_{0} f(\mathbf{Z}) \\
f_{i_{1} i_{2}}\left(Z_{i_{1}}, Z_{i_{2}}\right) & \equiv \mathcal{L}_{i_{1} i_{2}} f(\mathbf{Z})=\mathcal{P}^{i_{1} i_{2}} f(\mathbf{Z})-\mathcal{L}_{i_{1}} f(\mathbf{Z})-\mathcal{L}_{i_{2}} f(\mathbf{Z})-\mathcal{L}_{0} f(\mathbf{Z}) \\
f_{i_{1} \ldots i_{M}}\left(Z_{i_{1}}, Z_{i_{2}}, \ldots Z_{i_{M}}\right) & \equiv \mathcal{L}_{\mathbf{u}} f(\mathbf{Z})=\mathcal{P}^{\mathbf{u}} f(\mathbf{Z})-\sum_{\mathbf{v} \subset \mathbf{u}} \mathcal{L}_{\mathbf{v}} f(\mathbf{Z})
\end{aligned}
$$

where, $\mathbf{v}:=\left\{i_{1}, i_{2}, \ldots, i_{M}-1\right\}$. Therefore, the variational problem (Equation 6.3) can be restated as

$$
\int\left[f(\mathbf{Z})-f_{R}(\mathbf{Z})\right]^{2} d \rho(\mathbf{Z})
$$

is minimized with

$$
f_{R}(\mathbf{Z})=\left(\mathcal{L}_{0}+\sum_{i} \mathcal{L}_{i}+\sum_{i_{1}<i_{2}} \mathcal{L}_{i_{1} i_{2}}+\ldots+\sum_{i_{1}<\ldots<i_{M}} \mathcal{L}_{i_{1} \ldots i_{M}}\right) f(\mathbf{Z})
$$

The linear projection operators $(\mathcal{L})$ must have following properties

1. Idempotency:

$$
\mathcal{L}_{\mathbf{u}}^{2}=\mathcal{L}_{\mathbf{u}}
$$


2. Orthogonality:

$$
\mathcal{L}_{\mathbf{u}} \mathcal{L}_{\mathbf{v}}=0, \mathbf{v} \neq \mathbf{u}
$$

3. Resolution of the identity:

$$
\mathcal{L}_{0}+\sum_{i} \mathcal{L}_{i}+\sum_{i_{1}<i_{2}} \mathcal{L}_{i_{1} i_{2}}+\ldots+\sum_{i_{1}<\ldots<i_{M}} \mathcal{L}_{i_{1} \ldots i_{M}}=\mathbf{1}
$$

ANOVA-HDMR and Cut-HDMR can be used to find the component functions. Both the ANOVA-HDMR and Cut-HDMR satisfies the properties of linear operator as stated in Equations (6.9)-(6.11). Typically in ANOVA-HDMR, measure $\rho$ is taken as the ordinary Lebesgue measure as

$$
d \rho(\mathbf{Z})=d(\mathbf{Z})=\prod_{i=1}^{N} d Z_{i} .
$$

Correspondingly, the projection operator $\left(\mathcal{P}^{\mathbf{u}}\right)$ is given as

$$
\mathcal{P}^{\mathbf{u}}:=\int_{\Re^{N-M}} f(\mathbf{Z}) d \mathbf{Z}_{\mathcal{D} / \mathbf{u}},
$$

which requires $(N-M)$ dimensional integration. The component functions can be evaluated as

$$
\begin{aligned}
f_{0} & =\int_{\Re^{N}} f(\mathbf{Z}) d \mathbf{Z}, \\
f_{i}\left(Z_{i}\right) & =\int_{\Re^{N-1}} f(\mathbf{Z}) \prod_{j \neq i} d Z_{j}-f_{0}, \\
f_{i_{1} i_{2}}\left(Z_{i_{1}}, Z_{i_{2}}\right) & =\int_{\Re^{N-2}} f(\mathbf{Z}) \prod_{k \notin\left(i_{1}, i_{2}\right)} d Z_{k}-f_{i_{1}}\left(Z_{i_{1}}\right)-f_{i_{2}}\left(Z_{i_{2}}\right)-f_{0}, \\
\ldots &
\end{aligned}
$$


Finding each component functions are computationally intensive as one needs to perform high dimensional integration, e.g., Monte-Carlo integration which requires large number of function evaluations. Such a computational complexity can be reduced using the Cut-HDMR. In Cut-HDMR, the measure $\rho$ is taken as Dirac measure located at reference point $\overline{\mathbf{Z}}:=\left\{\bar{Z}_{1}, \bar{Z}_{2}, \ldots, \bar{Z}_{N}\right\}$ given as

$$
d \rho(\mathbf{Z})=\prod_{i=1}^{N} \delta\left(Z_{i}-\bar{Z}_{i}\right) d Z_{i} .
$$

Correspondingly, the projection operator is described as

$$
\mathcal{P}^{\mathbf{u}}:=\left.f(\mathbf{Z})\right|_{\mathbf{Z}=\left(\mathbf{Z}_{\mathbf{u}}, \overline{\mathbf{Z}}\right)},
$$

which is $M$ dimensional function. The component functions are evaluated at reference (cut) point, cut-lines, cut-planes and hyperplanes and are given as

$$
\begin{aligned}
f_{0} & =\left.f(\mathbf{Z})\right|_{\mathbf{z}=\overline{\mathbf{Z}}}, \\
f_{i}\left(Z_{i}\right) & =\left.f(\mathbf{Z})\right|_{\mathbf{z}=\left(Z_{i}, \overline{\mathbf{Z}}\right)}, \\
f_{i_{1} i_{2}}\left(Z_{i_{1}}, Z_{i_{2}}\right) & =\left.f(\mathbf{Z})\right|_{\mathbf{Z}=\left(Z_{i_{1}}, Z_{i_{2}}, \overline{\mathbf{Z}}\right)}-f_{i_{1}}\left(Z_{i_{1}}\right)-f_{i_{2}}\left(Z_{i_{2}}\right)-f_{0} .
\end{aligned}
$$

In the above equation,

$$
\begin{aligned}
\overline{\mathbf{Z}} & =\left\{\bar{Z}_{1}, \bar{Z}_{2}, \ldots, \bar{Z}_{N}\right\}, \text { (Cut-point) } \\
\left(Z_{i}, \overline{\mathbf{Z}}\right) & =\left\{\bar{Z}_{1}, \ldots, \bar{Z}_{i-1}, Z_{i}, \bar{Z}_{i+1}, \ldots, \bar{Z}_{N}\right\}, \text { (Cut-lines) } \\
\left(Z_{i_{1}}, Z_{i_{2}}, \overline{\mathbf{Z}}\right) & =\left\{\bar{Z}_{1}, \ldots, \bar{Z}_{i_{1}-1}, Z_{i_{1}}, \bar{Z}_{i_{1}+1}, \ldots, \bar{Z}_{i_{2}-1}, Z_{i_{2}}, \bar{Z}_{i_{2}+1}, \ldots, \bar{Z}_{N}\right\}, \text { (Cut-plane) }
\end{aligned}
$$

Hence, for a Cut-HDMR, the input sampling domain can be defined as

$$
\Omega^{s} \equiv\left\{\overline{\mathbf{Z}},\left(Z_{i}, \overline{\mathbf{Z}}\right),\left(Z_{i_{1}}, Z_{i_{2}}, \overline{\mathbf{Z}}\right)\right\}
$$


For $s$ number of input sampling points of each input variable, the required number of function evaluations (model run)

$$
N_{f}=1+N(s-1)+\frac{N(N-1)(s-1)^{2}}{2}
$$

which increases polynomically with $N$ and $s$. In the present study, the Cut-HDMR formulation is adopted for the construction of surrogate model to reduce the computational complexity in the situations where repetitive finite element simulations are required. Therefore, the HDMR based surrogate model has been applied in construction of failure envelop of heterogeneous microstructure and two scale finite element analysis of nonlinear hyperelastic composites.

\subsection{Application 1: Determination of failure enve- lope for heterogeneous materials*}

The construction of the failure envelop of a heterogeneous material is taken as an illustrative example. Failure envelop of a microstructure offers feasibility of that material model against catastrophic failure while in operation. To construct the envelop for heterogeneous materials, feasibility function must be tested over the entire input parameter space. For complex heterogeneous materials, this boils down to multiple costly finite element simulations. We pose this problem as a feasibility analysis problem (Banerjee et al., 2010). Surrogate modeling will alleviate the necessity for multiple full field finite element simulations. We first describe the surrogate modeling methodology for generic feasibility analysis. Then we present the application of HDMR technique in the construction of feasible strain space for a heterogeneous

$*$ Reprinted from Computationally efficient black-box modeling for feasibility analysis, article in press, Ipsita Banerjee, Siladitya Pal, Spandan Maiti, Computers \& Chemical Engineering, Copyright 2010, with permission from Elsevier (see Appendix $G$ ) 
materials systems.

\subsubsection{HDMR formalism for general feasibility problem}

The problem definition of feasibility analysis, whether in process systems or in material design, is to determine for a given design whether the system is feasible to operate over the expected range of variation of the uncertain parameters. Given a nominal value of the uncertain parameter $\theta^{N}$, and the expected deviation $\Delta \theta^{+}, \Delta \theta^{-}$, the flexibility test problem (Halemane and Grossmann, 1983) for a given design $d$ consists of determining whether the inequalities $f_{j}(d, z, \theta) \leq 0, j \in J$, holds for all

$\theta \in T=\left[\theta \mid \theta^{L} \leq \theta \leq \theta^{U}\right]$. This problem is posed as a standard optimization problem by defining a scalar variable $u$, such that

$$
\begin{aligned}
\psi(d, \theta) & =\min _{z, u} u \\
\text { subject to } \quad f_{j}(d, z, \theta) & \leq u \quad j \in J
\end{aligned}
$$

If $\psi(d, \theta) \leq 0$ for all $\theta \in T$, the process is deemed feasible in the parameter range of interest: $T=\left[\theta \mid \theta^{L} \leq \theta \leq \theta^{U}\right]$. To determine design feasibility without relying on process model, HDMR is applied considering Problem (6.21) as a black box model. The evaluation of HDMR component function is performed according to Equation (6.2) resulting in the following expansion for a fixed value of $d$ :

$$
\begin{aligned}
f_{0} & =\psi(\bar{\theta}) \\
f_{i}\left(\theta_{i}\right) & =\psi\left(\bar{\theta}^{i}, \theta_{i}\right)-f_{0} \\
f_{i j}\left(\theta_{i}, \theta_{j}\right) & =\psi\left(\bar{\theta}^{i j}, \theta_{i}, \theta_{j}\right)-f_{i}\left(\theta_{i}\right)-f_{j}\left(\theta_{j}\right)-f_{0}
\end{aligned}
$$


The second order HDMR expression is then given by:

$$
\hat{\psi}(d, \theta)=f_{0}+\sum_{i=1}^{n} f_{i}\left(\theta_{i}\right)+\sum_{1 \leq i<j \leq n} f_{i j}\left(\theta_{i}, \theta_{j}\right)
$$

In summary, while applying HDMR methodology in addressing feasibility problems, the following steps are employed:

1. The uncertain parameters are identified and model formulation is written in terms of the uncertain parameters

2. HDMR table is created by solving the actual model at discrete parameter values and evaluating the functions given by Equation (6.22) as described below:

- The $f_{0}$ term is determined with a single model run of Problem (6.21) with all the uncertain parameters $(\theta)$ at their nominal values $\left(\theta^{N}\right)$.

- The first order function, $f_{i}\left(\theta_{i}\right)$, is calculated from $\psi\left(\bar{\theta}^{i}, \theta_{i}\right)$ by setting all the input variables except $\theta_{i}$ to their nominal values $\left(\theta^{N}\right)$ and performing a series of model runs with the input value of $\theta_{i}$ varying over its uncertain range $\left(\theta^{L} \leq \theta \leq \theta^{U}\right)$. The $f_{0}$ term is then subtracted from each model output to produce the function $f_{i}\left(\theta_{i}\right)$ as shown in Equation (6.22).

- The second order function, $f_{i, j}\left(\theta_{i}, \theta_{j}\right)$ is calculated by setting all the input variables, except $\theta_{i}$ and $\theta_{j}$, at their nominal values and obtaining the response of the model for different values of $\theta_{i}$ and $\theta_{j}$ covering the input surface space.

3. Once the table is created, the entire parameter space can be reconstructed by interpolation. The output response at any desired point $\mathbf{x}$ is obtained by first 
interpolating each of the HDMR expansion terms in the look-up tables with respect to the input query point; then summing up the interpolated function values from zeroth to the highest required order. For second order expansion the estimated output will be given by:

$$
\zeta_{\text {predicted }}=f_{0}+\sum_{i=1}^{n} f_{i}\left(\theta_{i}\right)+\sum_{1 \leq i<j \leq n} f_{i j}\left(\theta_{i}, \theta_{j}\right)
$$

To evaluate the performance of this technique, the interpolation results are compared with the actual model runs at the corresponding parameter value, and an error value is computed as follows:

$$
\xi=\frac{1}{N} \sqrt{\sum_{i=1}^{N}\left(\frac{\zeta_{\text {predicted }}^{i}-\zeta_{\text {actual }}^{i}}{\zeta_{\text {actual }}^{i}}\right)^{2}}
$$

where, $\mathrm{N}$ is the number of interpolation points, $\zeta_{\text {predicted }}^{i}$ is the value obtained using HDMR prediction and $\zeta_{\text {actual }}^{i}$ is the actual solution of the feasibility analysis problem.

\subsubsection{HDMR applied to failure envelop determination for a particulate composite system}

In the example, we demonstrate the application of HDMR technique for the feasibility analysis of a complex material model. The model is based on the design of a twophase heterogeneous material with circular particles of different shape and size (phase 2) inserted in a matrix (phase 1), as illustrated in Figure 6.1. The objective here is to determine the range of externally applied strain over which a particular materials design retains feasibility without failing. The model will be represented by a complex set of partial differential equations as elaborated below. The design parameter vector 
$\mathbf{d}$ contains material properties of the two phases as well as the geometric features, such as size and arrangement of particles. Given specific values of the design parameters $\mathbf{d}$ for this model, we want to investigate the range of the input parameters (externally applied strain in this case) over which the design does not fail. The uncertain input parameters are thus the three components of the strain tensor:

$$
\theta_{1}:=\varepsilon_{11}^{0} ; \quad \theta_{2}:=\varepsilon_{22}^{0} ; \quad \theta_{3}:=\varepsilon_{12}^{0} .
$$

The criterion for feasibility of the described model will be to satisfy the following failure condition (Fung (1965)):

$$
f(\mathbf{d}, \theta)=\sqrt{\sigma_{1}^{2}+\sigma_{2}^{2}-\sigma_{1} \sigma_{2}}-\sigma_{f} \leq 0
$$

where

$$
\begin{aligned}
& \sigma_{1}=\frac{\sigma_{11}+\sigma_{22}}{2}+\sqrt{\left(\frac{\sigma_{11}-\sigma_{22}}{2}\right)^{2}+\sigma_{12}^{2}}, \\
& \sigma_{2}=\frac{\sigma_{11}+\sigma_{22}}{2}-\sqrt{\left(\frac{\sigma_{11}-\sigma_{22}}{2}\right)^{2}+\sigma_{12}^{2}},
\end{aligned}
$$

with $\sigma_{i j}, i, j=1,2$ as the stress tensor components, and $\sigma_{f}$ as the failure strength of the designed material.

Output quantities are related to the input quantities through the following equations everywhere within the domain (Sadd, (2005)):

$$
\begin{array}{r}
\sigma_{i j}=\lambda u_{k, k} \delta_{i j}+\mu\left(u_{i, j}+u_{j, i}\right), \\
\mu u_{i, k k}+(\lambda+\mu) u_{k, k i}+F_{i}=0, \quad i, j=1,2
\end{array}
$$

and the input strain

$$
\varepsilon_{i j}^{0}=\left(u_{i, j}^{0}+u_{j, i}^{0}\right) / 2 . \quad i, j=1,2
$$


In the above equations, $\lambda$ and $\mu$ are constants, $u_{i}$ are the displacement components, while quantities with a superscript 0 denote boundary quantities. These equations need to be solved using advanced numerical techniques such as finite element method (FEM). Determining the feasible region will require multiple solutions of the problem mapping the entire range of the uncertain input parameter, which will be computationally extremely expensive. Instead, we present the use of HDMR methodology to extract similar information but with largely reduced model runs. The elements of the proposed methodology is represented by flow chart in Figure 6.2. The design examined here consists of a square domain of dimension $100 \mu m \times 100 \mu m$. The particle diameters are given as $30 \mu \mathrm{m}, 35 \mu \mathrm{m}$ and $25 \mu \mathrm{m}$. Phase 1 has the properties of epoxy with $\lambda^{(1)}=2.85 G P a$ and $\mu^{(1)}=1.22 G P a$, while phase 2 is considered to be A-glass with $\lambda^{(2)}=17.31 G P a$ and $\mu^{(2)}=20.33 G P a$. The failure strength of the design is taken as $\sigma_{f}=100 \mathrm{MPa}$.

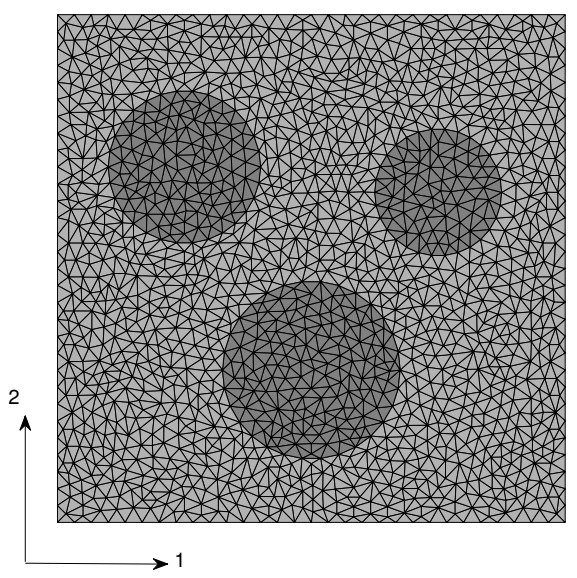

Figure 6.1: A representative design for a two phase material showing phase 1 material (epoxy matrix) in light gray and phase 2 material (A-glass particle) in dark gray. FEM mesh used for the simulations and the coordinate directions are also shown

To obtain the feasible domain for the input strain space, the parameter range for normal strains is taken as $\varepsilon_{11}^{0}=\varepsilon_{22}^{0} \in\left[\begin{array}{ll}-0.025 & 0.025\end{array}\right]$ and for shear strain as $\varepsilon_{12}^{0} \in$ $\left[\begin{array}{ll}-0.01875 & 0.01875\end{array}\right]$. Nominal point for the parameter space is given as $\varepsilon_{11}^{N}=\varepsilon_{22}^{N}=\varepsilon_{12}^{N}=0$. 


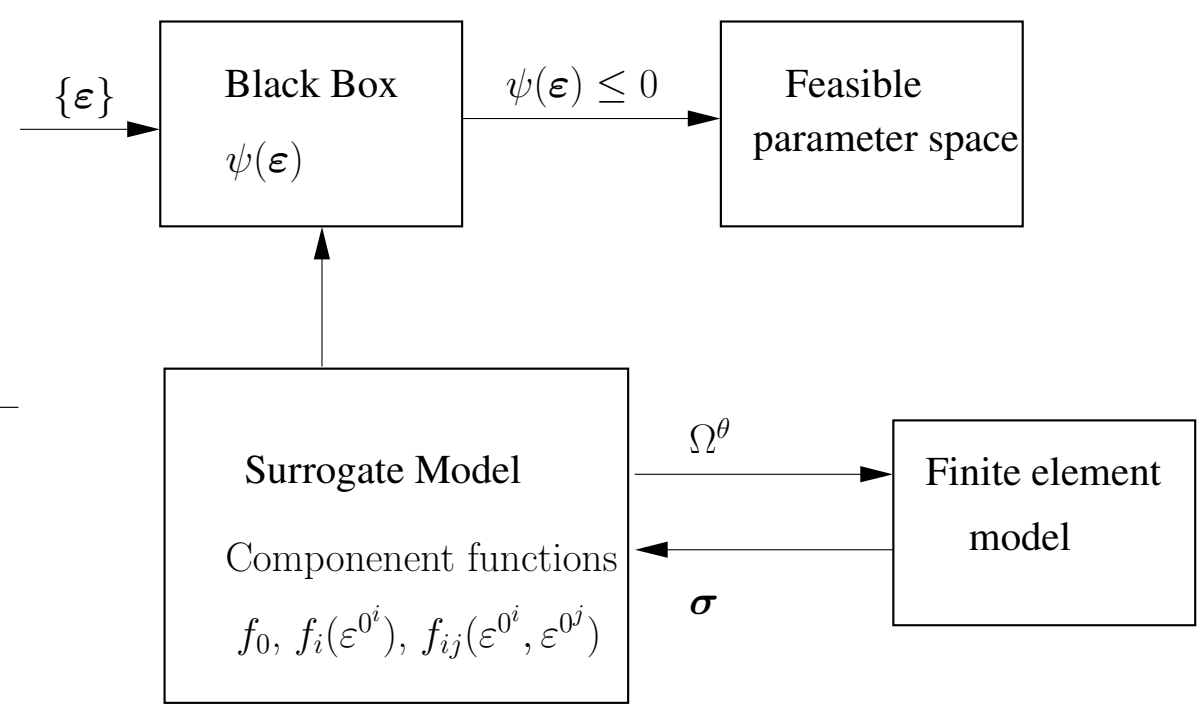

Figure 6.2: Flow chart showing basic elements of surrogate model based feasibility analysis of the heterogeneous materials.

The surrogate model (component functions) is constructed using the cut-HDMR approach. As mentioned earlier, for cut-HDMR the component functions are evaluated at the cut point, cut lines and cut surfaces. Correspondingly, the input parameter space $\Omega^{\theta}$ can be given as

$$
\begin{aligned}
\text { Cut Point }: & =\left(\varepsilon_{11}^{0^{N}}, \varepsilon_{22}^{0^{N}}, \varepsilon_{12}^{0^{N}}\right) \\
\text { Cut Lines } & :=\left(\varepsilon_{11}^{0^{i}}, \varepsilon_{22}^{0^{N}}, \varepsilon_{12}^{0^{N}}\right),\left(\varepsilon_{11}^{0^{N}}, \varepsilon_{22}^{0^{i}}, \varepsilon_{12}^{0^{N}}\right),\left(\varepsilon_{11}^{0^{N}}, \varepsilon_{22}^{0^{N}}, \varepsilon_{12}^{0^{i}}\right) \\
\text { Cut Planes }: & =\left(\varepsilon_{11}^{0^{i}}, \varepsilon_{22}^{0^{j}}, \varepsilon_{12}^{0^{N}}\right),\left(\varepsilon_{11}^{0^{N}}, \varepsilon_{22}^{0^{i}}, \varepsilon_{12}^{0^{j}}\right),\left(\varepsilon_{11}^{0^{i}}, \varepsilon_{22}^{0^{N}}, \varepsilon_{12}^{0^{j}}\right)
\end{aligned}
$$

An example of cut plane is described in Figure 6.3 which contains the cut point as well as two cut lines. The finite element simulations are performed over the above input parameter space. Each input parameter is discretized over 11 equal intervals. Thus, a total 331 finite element simulations were performed to construct second order HDMR component functions. We have only performed 2nd order HDMR calculations as it was shown to be very accurate in the previous example. Each finite element run takes $1146 \mathrm{cpu} s$ and the construction of the look up table by HDMR methodology thus requires $105.368 \mathrm{cpu}$ hrs. 


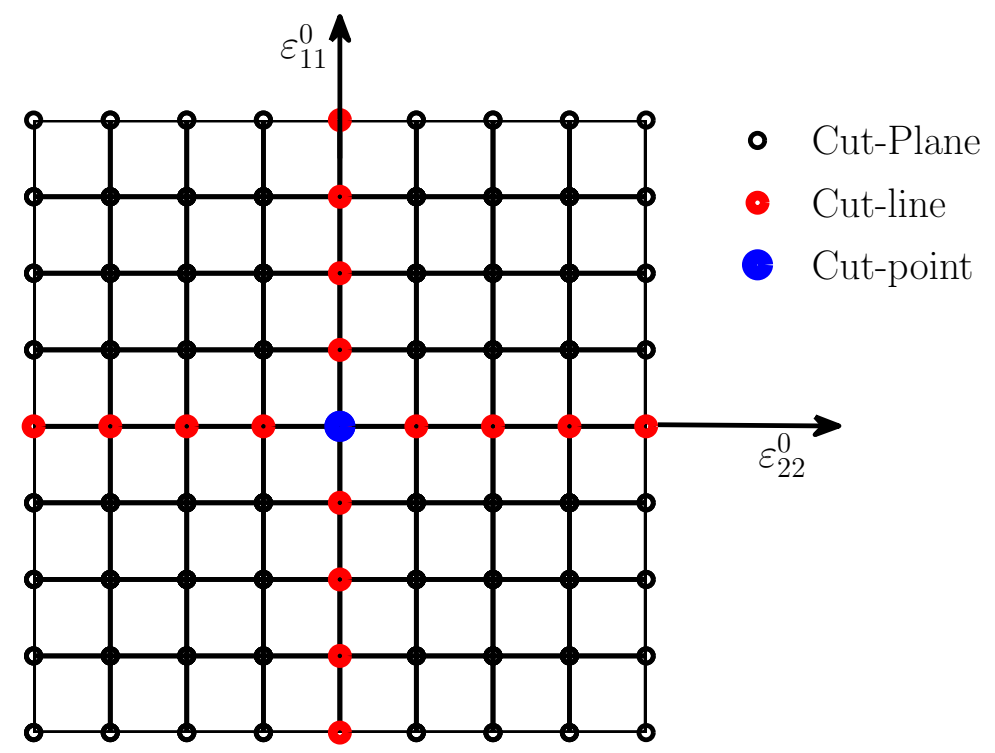

Figure 6.3: Grid of input sample points over which HDMR component functions are evaluated by performing FEM simulations.

Upon completion of the table generation computations, the model response over the entire 3-dimensional input parameter space is reconstructed by interpolation of the component functions at the query points on a $25 \times 25 \times 25$ grid that requires further $0.1525 \mathrm{cpu} s$. However, construction of the feasible space by actual finite element simulations on that grid requires $4974 \mathrm{cpu}$ hrs. Thus HDMR methodology can obtain the feasible region 47.2 times faster than the conventional model evaluation technique. This example shows the enormous computational savings to be accrued from the proposed methodology, particularly when the model complexity is high.

Figure (6.4) shows the predicted feasible region for $\varepsilon_{11}^{0}=\varepsilon_{22}^{0} \in\left[\begin{array}{ll}-0.025 & 0.025\end{array}\right]$ for a given shear strain $\varepsilon_{12}^{0}=0.00675$. The boundary of the feasible region (solid line) for this particular plane was calculated by solving the full FEM model on a $25 \times 25$ grid requiring $198.97 \mathrm{cpu}$ hrs. As illustrated in the figure, the HDMR method demonstrates excellent accuracy in capturing the actual feasible region of the given materials design. The error in prediction by Equation (6.25) is only $2.028 \times 10^{-4}$, denoting the 
high accuracy of our prediction. Thus, this particular example shows the computational efficiency and prediction accuracy of the proposed technique even with complex model formulations.

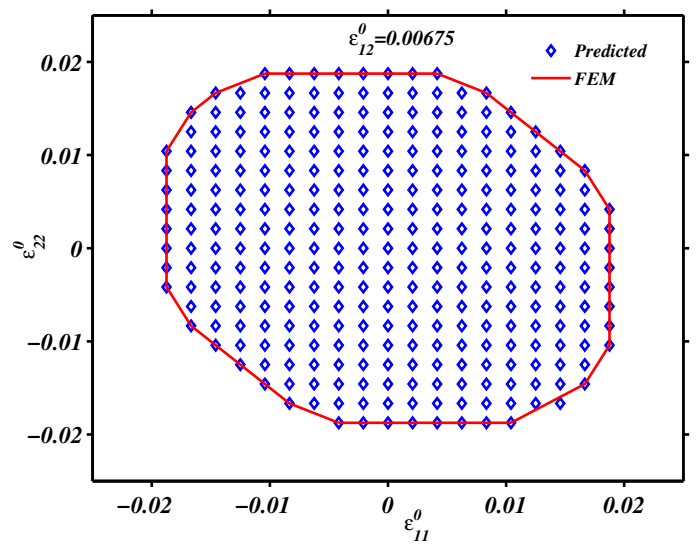

Figure 6.4: Comparison of FEM computed feasible region with the HDMR predictions

Two more projections from the 3-D feasible space are shown in Figure (6.5). Figure $6.5(\mathrm{a})$ corresponds to the feasible region for $\varepsilon_{12}^{0}$ and $\varepsilon_{11}^{0}$ keeping $\varepsilon_{22}^{0}=-0.018$, while Figure $6.5(\mathrm{~b})$ is for $\varepsilon_{12}^{0}$ and $\varepsilon_{22}^{0}$ keeping $\varepsilon_{11}^{0}=-0.016$. It can be noted from this figure that our prediction is highly accurate for all these planes.

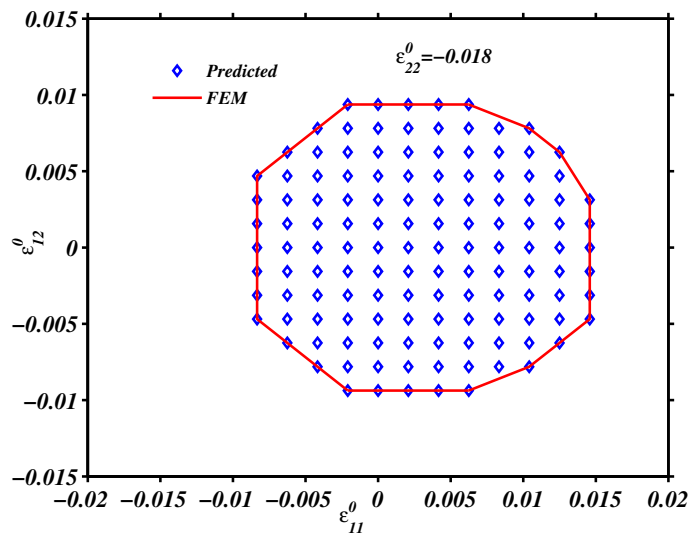

(a)

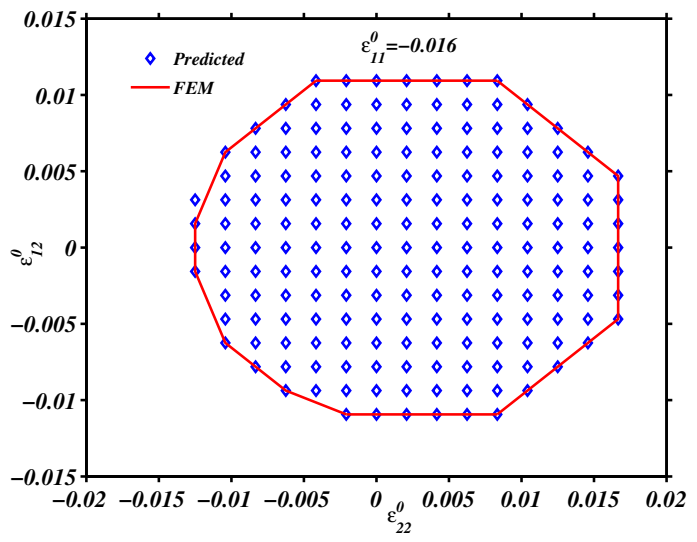

(b)

Figure 6.5: Comparison of HDMR predicted feasible region with FEM computed result (a) when $\varepsilon_{22}^{0}$ is fixed, and (b) when $\varepsilon_{11}^{0}$ is fixed. 


\subsection{Application 2: Efficient multiscale analysis of nonlinear hyperelastic materials undergoing la- rge deformation}

The two scale finite element method is widely used to find macroscopic behavior of a heterogeneous material. At the upper scale, the constitutive response at macroscopic Gauss point is evaluated concurrently performing finite element simulation (lower scale) of representative volume element attached to that point. Therefore, two scale finite element methods are computationally intensive especially when the material contains nonlinear hyperelastic constituents and undergoing large deformations. Furthermore, the multiple RVEs need to be solved for highly complex statistically heterogeneous microstructures. The main focus of the present analysis is to apply the HDMR based surrogate model to extract the constitutive behavior of the heterogeneous materials for a given deformation gradient at a macroscopic Gauss point by replacing the lower scale simulation. This surrogate model is constructed considering the representative volume element as a black box where the components of the macroscopic deformation gradient are viewed as input variables while average microscopic stress tensor as output. Applying homogeneous deformation boundary condition to the RVE and performing nonlinear large deformation finite element simulation over the input parameter space, we evaluate the HDMR component functions. Next, the average microscopic stress tensor for the arbitrary macroscopic deformation gradient is predicted from look-up table with minimal computation. To test the accuracy of such prediction, a full field finite element simulation is performed for the similar evolution of the macroscopic deformation gradient tensor. 


\subsubsection{Surrogate model based multiscale framework}

The purpose of the present multiscale model is to determine the locally averaged global constitutive behavior of nonlinear, possibly statistically heterogeneous material subjected to finite strain. In contrast to the concurrent multiscale framework, the present framework is based on two steps approach as shown in Figure. 6.6. Step-1 is the construction of a surrogate model. This can be achieved through extracting the average microscopic behavior over a sampled input variable space as dictated by Cut-HDMR expansion. Step-2 predicts the behavior from surrogate model for any arbitrary macroscopic deformation gradient at any macroscopic point resulting due to applied macroscale loading. Consequently, the primary components of this novel multiscale framework are given as (1) formulation of macroscale boundary value problem, (2) construction of surrogate model, and (3) formulation of microscopic boundary value problem. Large deformation kinematics are considered at both the scales. A periodic heterogeneous microstructure is considered. No energy dissipation mechanisms such as debonding between the material interfaces are allowed at both the scales.

\subsubsection{Macroscale formulation}

Let $\Omega_{0} \subset \Re^{n}$ be a $n$ dimensional macroscopic domain in its reference configuration, and $\partial \Omega_{0}$ denote the boundary of $\Omega_{0}$. The current configuration and its boundary are denoted as $\Omega$ and $\partial \Omega$, respectively. We assume that $\partial \Omega_{0}=\partial \Omega_{0 u} \cup \partial \Omega_{0 \sigma}$, and $\partial \Omega_{0 u} \cap$ $\partial \Omega_{0 \sigma}=\varnothing$ with subset $\Omega_{0 u}$ and $\Omega_{0 \sigma}$ indicate the portion where macroscopic displacement (u) and macroscopic traction (t) are prescribed, respectively. The material points at any instant is indicated as $\mathbf{X} \in \Re^{n}$. For a given macroscopic displacement field $\overline{\mathbf{u}}(\mathbf{X}) \in H_{1}\left(\Omega_{0}\right)$, the current position vector $\overline{\mathbf{x}}$ is given as $\overline{\mathbf{x}}(\mathbf{X})=\mathbf{X}+\overline{\mathbf{u}}(\mathbf{X})$. The 


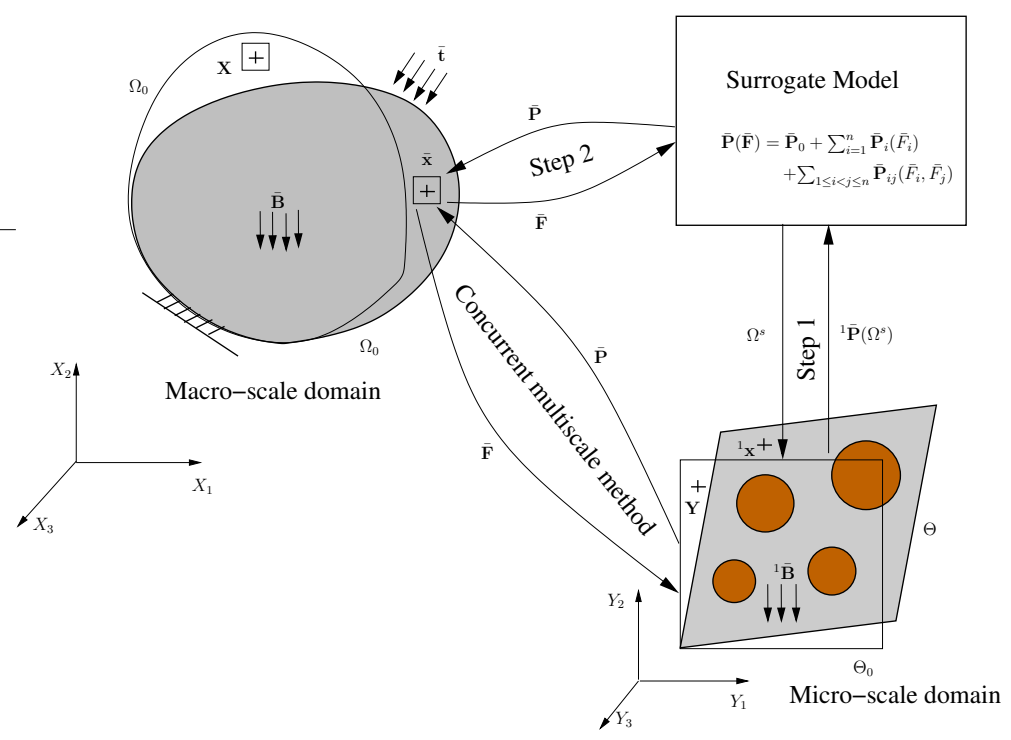

Figure 6.6: Schematics of Macro-and micro-scale boundary value problems and their coupling.

macroscopic deformation gradient tensor $\overline{\mathbf{F}}$ can be evaluated as $\overline{\mathbf{F}}=\nabla_{X} \overline{\mathbf{x}}$. The first Piola-Kirchhoff stress tensor (PK-I) at macro-scale $\overline{\mathbf{P}}$ is related to $\overline{\mathbf{F}}$ as $\overline{\mathbf{P}}=$ $\partial_{\overline{\mathbf{F}}} \bar{\Psi}$, where, $\bar{\Psi}$ represents the strain energy function of the homogenized material. Considering quasi-static deformation of the domain, the equilibrium conditions are expressed as

$$
\nabla \cdot \overline{\mathbf{P}}+\overline{\mathbf{B}}=\mathbf{0}, \quad \text { and } \quad \overline{\mathbf{P}} \overline{\mathbf{F}}^{T}=\left(\overline{\mathbf{P}} \overline{\mathbf{F}}^{T}\right)^{T}
$$

where, $\overline{\mathbf{B}}$ represent the body force in reference configuration. The boundary conditions are prescribed as $\mathbf{u}(\mathbf{X})=\overline{\mathbf{u}}(\mathbf{X}) \quad$ on $\quad \partial \Omega_{0 u}$ and $\overline{\mathbf{P}} \mathbf{N}=\overline{\mathbf{t}} \quad$ on $\quad \partial \Omega_{0 \sigma}$ where, $\mathbf{N}$ is outward normal at $\mathbf{X} \in \partial \Omega_{0 \sigma}$. The first Piola-Kirchhoff stress tensor $\overline{\mathbf{P}}$ can be related to Cauchy stress tensor $\boldsymbol{\sigma}$ by $\overline{\mathbf{P}}=J \boldsymbol{\sigma} \overline{\mathbf{F}}^{-1}$, where, $J=\operatorname{det}(\overline{\mathbf{F}})$, and the second Piola-Kirchhoff stress tensor $\overline{\mathbf{S}}$ as $\overline{\mathbf{P}}=\overline{\mathbf{F}} \overline{\mathbf{S}}$. For any admissible displacement field $\delta \mathbf{u} \in H_{0}^{1}\left(\Omega_{0}\right)$, the principle of virtual work states that

$$
\delta W_{\text {int }}(\mathbf{u}, \delta \mathbf{u})-\delta W_{\text {ext }}(\mathbf{u}, \delta \mathbf{u})=\mathbf{0}
$$


where internal virtual work $\delta W_{\text {int }}$ and external virtual work $\delta W_{\text {ext }}$ can be found in material description as

$$
\begin{aligned}
\delta W_{\text {int }}(\mathbf{u}, \delta \mathbf{u}) & =\int_{\Omega_{0}} \overline{\mathbf{S}}: \delta \overline{\mathbf{E}} \partial \Omega=\int_{\Omega_{0}} \overline{\mathbf{P}}: \nabla_{X}(\delta \mathbf{u}) \partial \Omega \\
\delta W_{\text {ext }}(\mathbf{u}, \delta \mathbf{u}) & =\int_{\Omega_{0}} \overline{\mathbf{B}} \cdot \delta \mathbf{u} \partial \Omega+\int_{\partial \Omega_{0}} \overline{\mathbf{t}} \cdot \delta \mathbf{u} \partial \Gamma
\end{aligned}
$$

In the above equation, $\overline{\mathbf{S}}, \overline{\mathbf{B}}$ and $\overline{\mathbf{t}}$ denote the second-Piola Kirchoff stress tensor, body force and traction applied at the boundary, respectively. The macroscopic Green-Lagrange strain tensor is given as $\overline{\mathbf{E}}=\frac{1}{2}(\overline{\mathbf{C}}-\mathbf{I})=\left(\overline{\mathbf{F}}^{T} \overline{\mathbf{F}}-\mathbf{I}\right) / 2$. An incremental/iterative solution technique is adopted to solve the above nonlinear equation. Linearization of the above equation offers

$$
\delta W_{\text {int }}(\mathbf{u}, \delta \mathbf{u})-\delta W_{\text {ext }}(\mathbf{u}, \delta \mathbf{u})+D_{\Delta \mathbf{u}} \delta W_{\text {int }}(\mathbf{u}, \delta \mathbf{u})-D_{\Delta \mathbf{u}} \delta W_{\text {ext }}(\mathbf{u}, \delta \mathbf{u})=0
$$

where, $D(\cdot)$ represents the Directional derivative (Gateaux operator). Using the variation of $\overline{\mathbf{E}}$ as

$$
\delta \overline{\mathbf{E}}=\frac{1}{2}\left[\overline{\mathbf{F}}^{T} \nabla_{X}(\delta \mathbf{u})+\nabla_{X}(\delta \mathbf{u})^{T} \overline{\mathbf{F}}\right]
$$

in Equation (6.34), the directional derivative of the internal virtual work can be obtained as

$$
D_{\Delta \mathbf{u}} \delta W_{\text {int }}(\mathbf{u}, \delta \mathbf{u})=\int_{\Omega_{0}} \nabla_{X}(\delta \mathbf{u}): \nabla_{X}(\Delta \mathbf{u}) \overline{\mathbf{S}} \partial \Omega+\overline{\mathbf{F}}^{T} \nabla_{X}(\delta \mathbf{u}): \overline{\mathbb{C}}: \overline{\mathbf{F}}^{T} \nabla_{X}(\Delta \mathbf{u}) \partial \Omega
$$

where $\overline{\mathbb{C}}$ is the fourth order homogenized material elasticity tensor. In case of a macroscopic heterogeneous material, $\bar{\Psi}$ is unknown in general. The tangent moduli (elasticity tensor) $\overline{\mathbb{C}}$ can not be expressed in closed form. Hence, we need to extract the homogenized macroscopic stress-deformation gradient $(\overline{\mathbf{P}}$ vs $\overline{\mathbf{F}})$ relationship. In a concurrent multiscale approach as indicated in Figure 6.6, we simulate the representative volume element for macroscopic deformation gradient at microlevel during 
each macroscopic Newton-Raphson iteration. As mentioned earlier, the macroscopic stress deformation gradient relationship at each Gauss point for each iteration will be extracted from a fully equivalent operational model (see Figure. 6.6). This surrogate model is generated through precomputation of the average response of the microstructure over a sampling space of $\overline{\mathbf{F}}$ indicated by $\Omega^{s}$ in Figure. 6.6. The detail formulation of surrogate model will be described in the next section.

\subsubsection{Surrogate model of RVE using HDMR formulation}

In this multiscale finite element framework, the HDMR based surrogate model is constructed to correlate the input-output relationship of a RVE, where the components of macroscopic deformation gradient tensor $\overline{\mathbf{F}}$ are considered as the input variables and components of first Piola-Kirchhoff stress tensor $\overline{\mathbf{P}}$ are considered as the output of the system. A HDMR based approach as described in Section 6.2 is applied to correlate the input-output (IO) relationship considering the RVE as a black-box. As mentioned earlier, HDMR expresses $\overline{\mathbf{P}}$ as a finite hierarchical correlated function expansion in terms of macroscopic deformation gradient $\overline{\mathbf{F}}$ :

$$
\overline{\mathbf{P}}(\overline{\mathbf{F}}) \approx \overline{\mathbf{P}}_{0}+\sum_{i=1}^{n} \overline{\mathbf{P}}_{i}\left(\bar{F}_{i}\right)+\sum_{1 \leq i<j \leq n} \overline{\mathbf{P}}_{i j}\left(\bar{F}_{i}, \bar{F}_{j}\right)
$$

where, the zeroth order component function $\overline{\mathbf{P}}_{0}$ is a constant representing the response of $\overline{\mathbf{P}}(\overline{\mathbf{F}})$ at the reference point denoted as $\overline{\mathbf{F}}^{R}$. The first order component function $\overline{\mathbf{P}}_{i}\left(\bar{F}_{i}\right)$ gives the independent contribution to $\overline{\mathbf{P}}(\overline{\mathbf{F}})$ when $i^{\text {th }}$ input variable or each component of deformation gradient acting alone. $\overline{\mathbf{P}}_{i j}$ gives the pair correlated contribution of the component of deformation gradient to the output $\overline{\mathbf{P}}(\overline{\mathbf{F}})$. Furthermore, it is assumed any residual higher order $(>2)$ correlated contribution of the components of deformation gradient to the output vanishes. There are two commonly used HDMR expansions namely Cut- and RS-HDMR corresponding to 
ordered and random sampling, respectively. Cut-HDMR expresses $\overline{\mathbf{P}}(\overline{\mathbf{F}})$ in reference to the specified cut point $\overline{\mathbf{F}}^{R}$ in desired domain of input variable represented by $\Omega^{s}$. Moreover, RS-HDMR depends on the average value of $\overline{\mathbf{P}}(\overline{\mathbf{F}})$ over the whole domain $\Omega^{s}$. Applying the Cut-HDMR, the component functions with respect to the reference point $\overline{\mathbf{F}}^{R}$ given as

$$
\begin{aligned}
\overline{\mathbf{P}}_{0} & ={ }^{1} \overline{\mathbf{P}}\left(\overline{\mathbf{F}}^{R}\right), \\
\overline{\mathbf{P}}_{i}\left(\bar{F}_{i}\right) & ={ }^{1} \overline{\mathbf{P}}\left(F_{i}, \overline{\mathbf{F}}_{i}^{R}\right)-\overline{\mathbf{P}}_{0}, \\
\overline{\mathbf{P}}_{i j}\left(\bar{F}_{i}, \bar{F}^{j}\right) & ={ }^{1} \overline{\mathbf{P}}\left(F_{i}, F_{j}, \overline{\mathbf{F}}_{i j}^{R}\right)-\overline{\mathbf{P}}_{i}\left(\bar{F}_{i}\right)-\overline{\mathbf{P}}_{j}\left(\bar{F}_{j}\right)-\overline{\mathbf{P}}_{0},
\end{aligned}
$$

where, ${ }^{1} \overline{\mathbf{P}}$ is the first Piola-Kirchhoff stress tensor need to be evaluated by performing RVE simulations over the sampling space $\Omega^{s}$. For a cut-HDMR the sampling space, $\Omega^{s}$ consists of the cut-point, cut-lines and cut planes as defined following

$$
\begin{aligned}
\overline{\mathbf{F}}^{R} & =\left(\bar{F}_{1}^{R}, \ldots, \bar{F}_{i-1}^{R}, \bar{F}_{i}^{R}, \bar{F}_{i+1}^{R}, \ldots, \bar{F}_{n}^{R}\right), \\
\left(F_{i}, \overline{\mathbf{F}}_{i}^{R}\right) & =\left(\bar{F}_{1}^{R}, \ldots, \bar{F}_{i-1}^{R}, \bar{F}_{i}, \bar{F}_{i+1}^{R}, \ldots, \bar{F}_{n}^{R}\right), \\
\left(F_{i}, F_{j}, \overline{\mathbf{F}}_{i j}^{R}\right) & =\left(\bar{F}_{1}^{R}, \ldots, \bar{F}_{i-1}^{R}, \bar{F}_{i}, \bar{F}_{i+1}^{R}, \ldots, \bar{F}_{j-1}^{R}, \bar{F}_{j}, \bar{F}_{j+1}^{R}, \ldots, \bar{F}_{n}^{R}\right) .
\end{aligned}
$$

In the above equations, $n$ representing the number of input variables. Numerical data tables are constructed for the component functions and the value of $\overline{\mathbf{P}}(\overline{\mathbf{F}})$ for any arbitrary point $\overline{\mathbf{F}}$ are determined through the low dimensional interpolation over $\overline{\mathbf{P}}_{i}\left(\bar{F}_{i}\right)$ and $\overline{\mathbf{P}}_{i j}\left(\bar{F}_{i}, \bar{F}_{j}\right)$. For a two dimensional finite strain analysis, there are four independent components of deformation gradient i.e., $n=\operatorname{dim}(\overline{\mathbf{F}})=4$. Consequently, it requires $3-8 s+6 s^{2}$ number of function evaluations $\left(N_{f}\right)$ for $s$ number of sampling point for each input variable. In contrast, traditional $s^{4}$ function evaluations required to construct a look-up table for performing multidimensional interpolation. Moreover, for a three dimensional analysis, $n=9$, which offers $N_{f}=28-63 s+36 s^{2}$ compared to $s^{9}$ model evaluation. Finally, to construct the look-up table for given input space $\Omega^{s}$, the 
average microscopic behavior of the RVE must be computed. Hence, a microscopic boundary problem is formulated in the next section.

\subsubsection{Microscale formulation}

Let $\Theta_{0} \subset \Re^{n}(n=1,2$ or 3$)$ be a RVE of the microstructure around a macroscopic point $\mathbf{X}$ (Figure. 6.6). Let $\mathbf{Y}$ be the microscale coordinate vector where, $\mathbf{Y}=\mathbf{X} / \epsilon$, with the parameter $\epsilon$ is very small compared to the macro-scale domain denoted by $\Omega_{0}$. In the microscale, we assume the existence of a strain energy function $\Psi^{(k)}$ of each phase $(k)$ in the microstructure. Correspondingly, the first Piola-Kirchhoff stress at each phases can be related to the microscopic deformation gradient as ${ }^{1} \mathbf{P}^{(k)}=\partial_{1} \mathbf{F} \Psi^{(k)}$, where, ${ }^{1} \mathbf{F}=\nabla_{X}{ }^{1} \mathbf{u}+1$, with ${ }^{1} \mathbf{u}$ is the microscopic displacement. The weak form at microscale can be formulated as; Find ${ }^{1} \mathbf{u} \in H^{1}\left(\Theta_{0}\right)$ satisfying ${ }^{1} \mathbf{u}={ }^{1} \overline{\mathbf{u}}$ on $\partial \Theta_{0 u}$ such that

$$
\int_{\Theta_{0}}{ }^{1} \mathbf{P}: \nabla_{X}(\delta \mathbf{u}) \partial \Theta=\int_{\Theta_{0}}{ }^{1} \mathbf{B} \cdot \delta \mathbf{u} \partial \Theta+\int_{\partial \Theta_{0}}{ }^{1} \mathbf{t} \cdot \delta \mathbf{u} \partial \Gamma, \quad \forall \delta \mathbf{u} \in H_{0}^{1}\left(\Theta_{0}\right),
$$

where ${ }^{1} \mathbf{B}$ are the local body forces and ${ }^{1} \mathbf{t}$ are the applied tractions. To solve this BVP, we need to specify appropriate boundary conditions. At microscopic length scale, we assume the current position of the material points is the superposition of an average field and a fluctuating field ${ }^{1} \tilde{\mathbf{u}}(\mathbf{Y})$ aroused due to the presence of heterogeneity as ${ }^{1} \mathbf{x}=\overline{\mathbf{F}} \mathbf{Y}+{ }^{1} \tilde{\mathbf{u}}(\mathbf{Y})$. Correspondingly, the deformation gradient at microscale given as ${ }^{1} \mathbf{F}=\overline{\mathbf{F}}+\nabla_{X}{ }^{1} \tilde{\mathbf{u}}(\mathbf{Y})$. A finite element method as described in Appendix $\mathrm{D}$ is used to solve the microscopic boundary value problem subjected to specific boundary conditions. After solving the microscopic boundary value problem, a homogenization procedure is performed to evaluated the average microscopic stress tensor for the given macroscopic deformation gradient. The average first Piola-Kirchhoff microstress ${ }^{1} \overline{\mathbf{P}}$ 
and average macro-deformation ${ }^{1} \overline{\mathbf{F}}$ of the microstructure are defined as

$$
{ }^{1} \overline{\mathbf{P}}:=\frac{1}{\Theta_{0}} \int_{\partial \Theta_{0}}{ }^{1} \mathbf{t} \otimes Y d \Gamma, \quad \text { and } \quad{ }^{1} \overline{\mathbf{F}}=\frac{1}{\Theta_{0}} \int_{\partial \Theta_{0}}{ }^{1} \mathbf{x} \otimes \mathbf{N} d \Gamma
$$

in terms of boundary data of tractions ${ }^{1} \mathbf{t}$ and deformation ${ }^{1} \mathbf{x}$ on the surface of the RVE. An averaging theorem as formulated by Hill (1972) requires the average of the microscopic stress power to be equal to the macroscopic stress power i.e., ${ }^{1} \overline{\mathbf{P}}$ : ${ }^{1} \dot{\overline{\mathbf{F}}}=\int_{d \Theta_{0}}{ }^{1} \mathbf{P}:{ }^{1} \dot{\mathbf{F}} d \Gamma$. Using the equilibrium condition and the identity, ${ }^{1} \mathbf{P}:{ }^{1} \mathbf{F}=$ $\operatorname{Div}\left[{ }^{1} \dot{\mathbf{x}} \cdot{ }^{1} \mathbf{P}\right]-\operatorname{Div}[\mathbf{P}] \cdot{ }^{1} \dot{\mathbf{x}}$ along with Gauss theorem, the above equation can be reformulated as

$$
{ }^{1} \overline{\mathbf{P}}:{ }^{1} \dot{\overline{\mathbf{F}}}=\frac{1}{\left|\Theta_{0}\right|} \int_{\partial \Theta_{0}}{ }^{1} \mathbf{t} \cdot{ }^{1} \dot{\mathbf{x}} d \Gamma
$$

in terms of the power of the tractions on the surface $\partial \Theta_{0}$. Moreover, one expects the property

$$
\left({ }^{1} \overline{\mathbf{P}}^{1} \overline{\mathbf{F}}^{T}\right)^{T}={ }^{1} \overline{\mathbf{P}}^{1} \overline{\mathbf{F}}^{T}
$$

for overall macroscopic stress in order to satisfy the Kirchhoff's macro-stress. The boundary conditions to be considered such that the averaging theorem and symmetric condition are satisfied. After mathematical manipulation, ${ }^{1} \overline{\mathbf{F}}$ can be written as

$$
{ }^{1} \overline{\mathbf{F}}=\overline{\mathbf{F}}+\frac{1}{\Theta_{0}} \int_{\partial \Theta_{0}}{ }^{1} \tilde{\mathbf{u}}(\mathbf{Y}) \otimes \mathbf{N} d \Gamma
$$

where $\overline{\mathbf{F}}$ is the macroscopic deformation gradient such that $\overline{\mathbf{F}}={ }^{1} \overline{\mathbf{F}}$, which leads to

$$
\frac{1}{\Theta_{0}} \int_{\partial \Theta_{0}}{ }^{1} \tilde{\mathbf{u}}(\mathbf{Y}) \otimes \mathbf{N} d \Gamma=0
$$

where, $\mathbf{N}$ is the unit outward normal on $\Theta_{0}$. The above equation can be satisfied for following boundary conditions at microscale

$$
{ }^{1} \tilde{\mathbf{u}}(\mathbf{Y})=\mathbf{0} \quad \text { on } \quad \partial \Theta_{0}, \text { and } \quad{ }^{1} \tilde{\mathbf{u}}^{+}(\mathbf{Y})={ }^{1} \tilde{\mathbf{u}}^{-}(\mathbf{Y}) \quad \text { on } \quad \partial \Theta_{0}
$$


The first condition offers the boundary constraint in terms of macroscopic deformation $\overline{\mathbf{F}}$ as

$$
{ }^{1} \mathbf{x}=\overline{\mathbf{F}} \mathbf{Y} \quad \text { on } \quad \partial \Theta_{0}
$$

which defines a linear deformation on the boundary $\partial \Theta_{0}$ of the representative volume. Substituting Equation (6.48) in Equation (6.43) confirms that the averaging theorem is a priori satisfied. Furthermore, Equation (6.42) and (6.48) along with equilibrium

of couples $\int_{\partial \Theta_{0}}{ }^{1} \mathbf{x} \otimes{ }^{1} \mathbf{t}=\mathbf{0}$, offers the expression which confirms the symmetry of the macroscopic Kirchhoff stresses (Miche, 2003; Yvonnet and He, 2007)

\subsubsection{Numerical examples}

Numerical examples are presented in this section to illustrate the performance of the proposed scheme. Particulate composite as well as short fiber composite made of nonlinear constituent materials are considered for the macroscopic domain. A perfect bonding between the different constitutive phases is assumed. The representative volume elements are discretized with four-noded quadrilateral elements.

\subsubsection{Particulate composites}

An example of heterogeneous material, a model particulate composite with a particle volume fraction of $24.5 \%$, is considered here. The particulate composite consists of a softer matrix and harder particles (indicated by darker regions) with different radii and stiffness. Figure. 6.7 represents a representative volume element (RVE) of this material that we will consider for our study. The constitutive response for the matrix as well as the particles is considered to be of compressible Neo-Hookean type, whose 


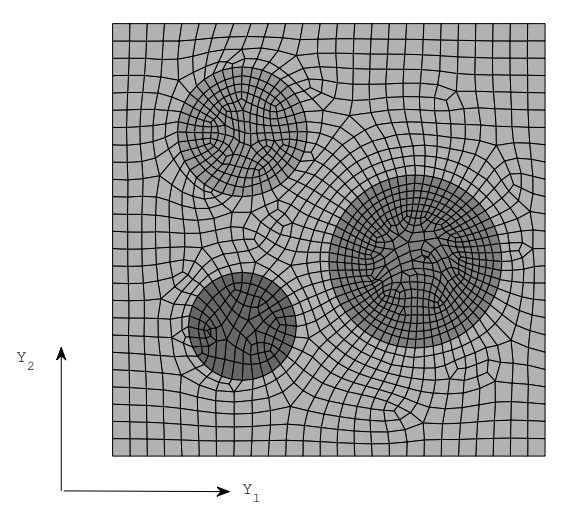

Figure 6.7: Representative volume element consisting softer matrix and harder particles of different radii as well as different stiffness. Particles are denoted by darker regions, darker shade denoting higher stiffness.

strain energy function can be represented as

$$
\Psi^{(k)}=\frac{\mu^{(k)}}{2}\left(I_{c}-3\right)-\mu^{(k)} \ln J+\frac{\lambda^{(k)}}{2}(\ln J)^{2}
$$

where, $\mu^{(k)}$ and $\lambda^{(k)}$ are the material constant for the $k^{t h}$ constituents, $I_{c}=\operatorname{tr}(\mathbf{C})$, is first invariant of $\mathbf{C}$ and $J^{2}=I I_{c}$. The material properties of the matrix and particles are listed in Table 6.1.

Table 6.1: Material properties

\begin{tabular}{|c|c|c|c|}
\hline \hline Constituents & Phase & $\lambda^{(k)}(M P a)$ & $\mu^{(k)}(M P a)$ \\
\hline Matrix & 1 & 12.1666 & 8 \\
Particle 1 & 2 & 1216.66 & 800 \\
Particle 2 & 3 & 6083.33 & 4000 \\
Particle 3 & 4 & 12166.66 & 8000 \\
\hline
\end{tabular}

The objective of the present study is to predict the macroscopic first-Piola Kirchhoff stress tensor at a Gauss point, that is represented by the above mentioned RVE at the microscale, for a given macroscopic deformation gradient resulting from an arbitrary loading on the structure. For the present example, the maximum macroscopic deformation gradient tensor is considered as $\overline{\mathbf{F}}=\left\{\begin{array}{llll}\bar{F}_{11} & \bar{F}_{22} & \bar{F}_{12} & \bar{F}_{21}\end{array}\right\}=\left\{\begin{array}{llll}1.3 & 1.3 & 0.1 & 0.1\end{array}\right\}$ resulting in a finite strain given as $\mathbf{E}=\left\{E_{11} E_{22} E_{12}\right\}=\left\{\begin{array}{ll}0.35 & 0.350 .13\end{array}\right.$ Resulting com- 
plex stress (first Piola-Kirchhoff stress) pattern at the microscale due to the presence of heterogeneity are illustrated in Figure. 6.8.

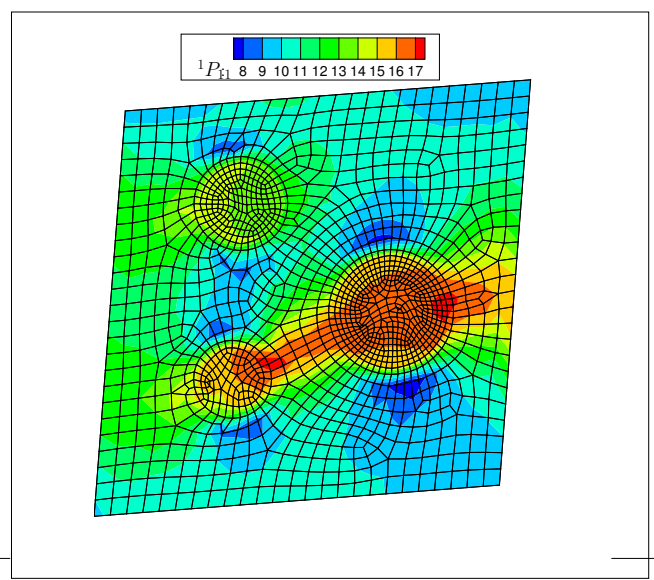

(a)

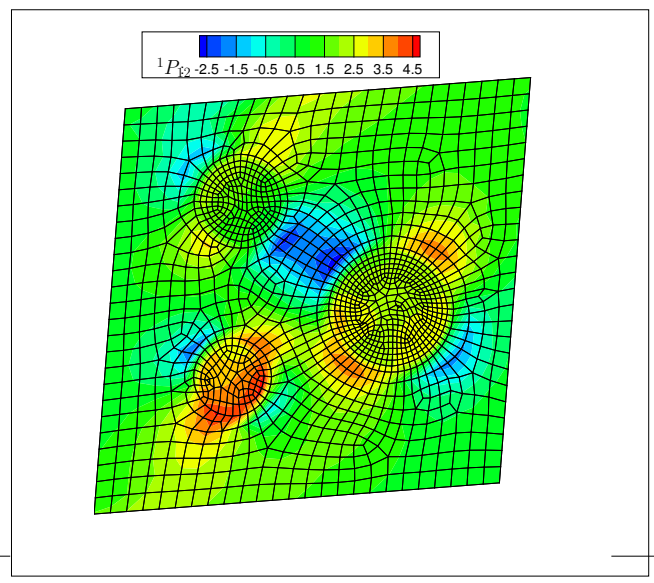

(c)

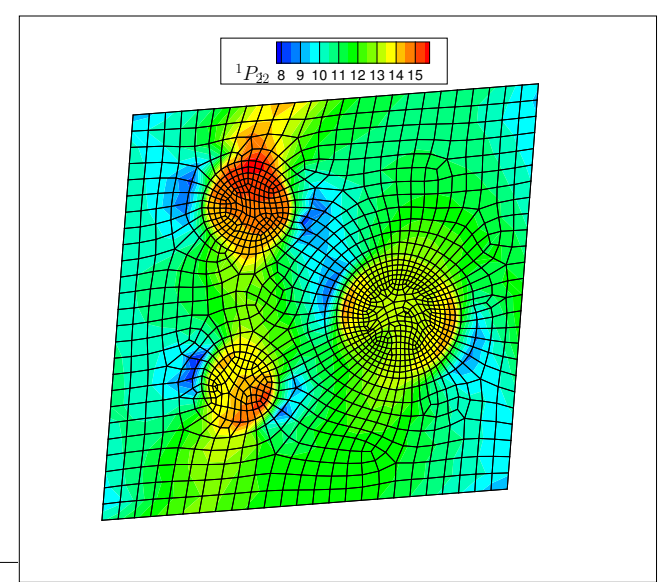

(b)

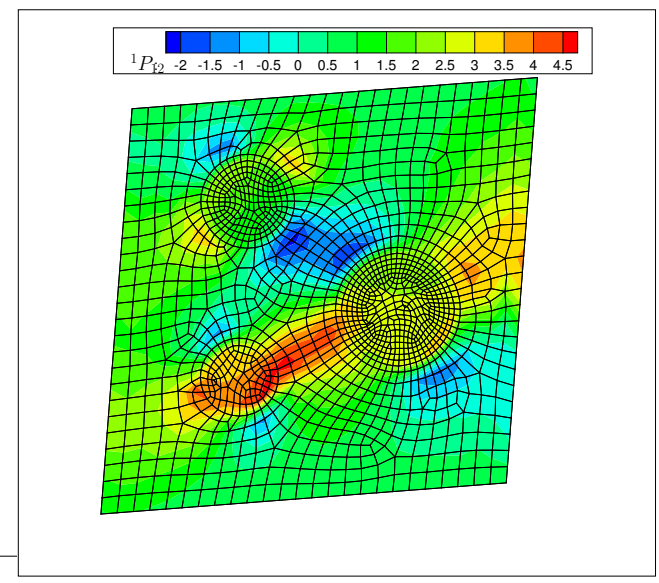

(d)

Figure 6.8: Stress contour of (a) ${ }^{1} P_{11}$, (b) ${ }^{1} P_{22}$, (c) ${ }^{1} P_{12}$, and (d) ${ }^{1} P_{21}$ for the RVE when subjected to macroscopic deformation gradient $\bar{F}_{11}=\bar{F}_{22}=1.3$, and $\bar{F}_{12}=\bar{F}_{21}=0.1$

In order to predict the macroscopic stress for any arbitrary deformation history, first the HDMR component functions are generated for the entire range of the considered deformation gradients. In this analysis, the undeformed state of the RVE is considered as the reference condition which is denoted as $\overline{\mathbf{F}}^{R}=\left\{\begin{array}{llll}1.0 & 1.0 & 0.0 & 0.0\end{array}\right\}$ (see Equation (6.40)). Correspondingly, the zeroth order stress tensor is given as ${ }^{1} \overline{\mathbf{P}}\left(\overline{\mathbf{F}}^{R}\right)=$ 
$\left\{\begin{array}{llll}0.0 & 0.0 & 0.0 & 0.0\end{array}\right\}$. Next, each component of the macroscopic deformation gradient is sampled over 11 equally spaced points, i.e., $s=11$. RVE simulations are performed over input parameter space as specified by Equation (6.40) resulting in 641 finite element runs to obtain zeroth, first and second order components of the microscopic stress tensor ${ }^{1} \overline{\mathbf{P}}$. Simulated first order and second order components of ${ }^{1} \bar{P}_{11}$ are illustrated in Figure. 6.9 and Figure. 6.10, respectively.
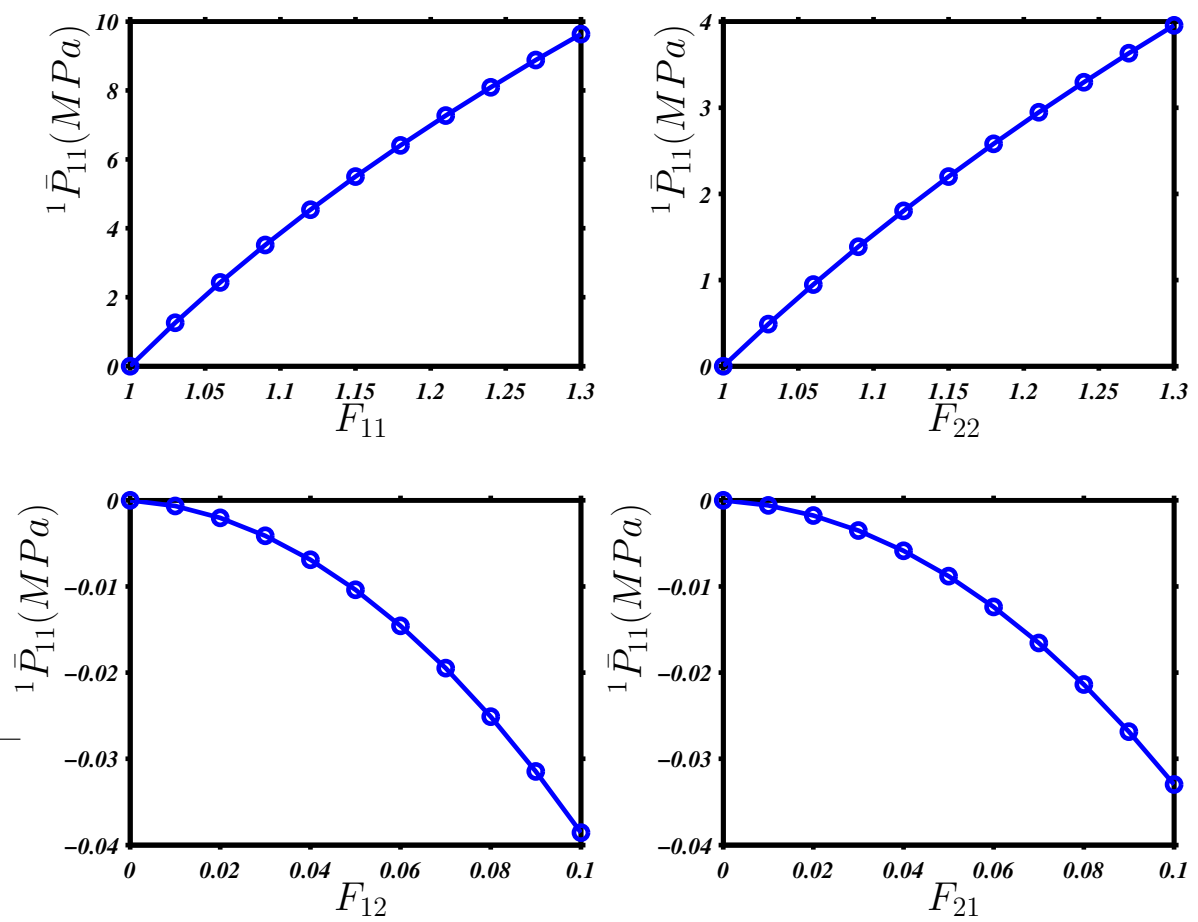

Figure 6.9: Simulated first order components of average microstress $\left({ }^{1} \bar{P}_{11}\right)$ with respect to the input variables.

The average macroscopic stress tensor resulting from an arbitrary macroscopic deformation gradient evolution can be obtained from Equation (6.38). The response from a full field finite element simulation performed for the similar evolution of macroscopic deformation gradient is taken as the reference case for the comparison purpose. Note, the loading paths that will be presented here do not belong to the sampling space (cut-lines and cut-surfaces) considered for the construction of surrogate model. In the 


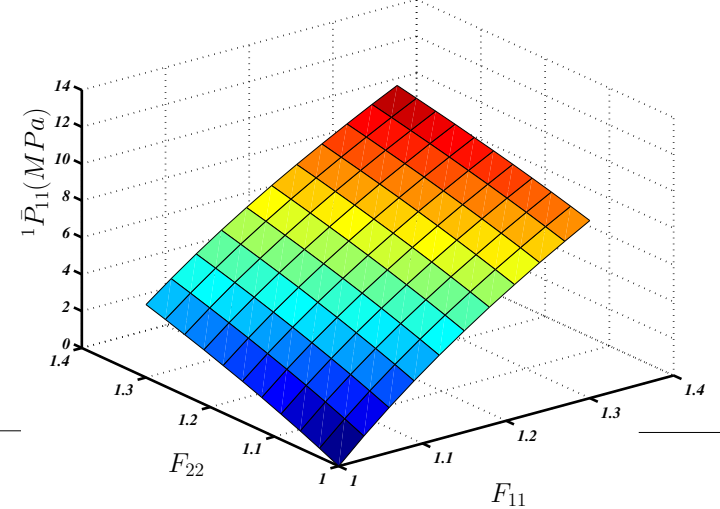

(a)

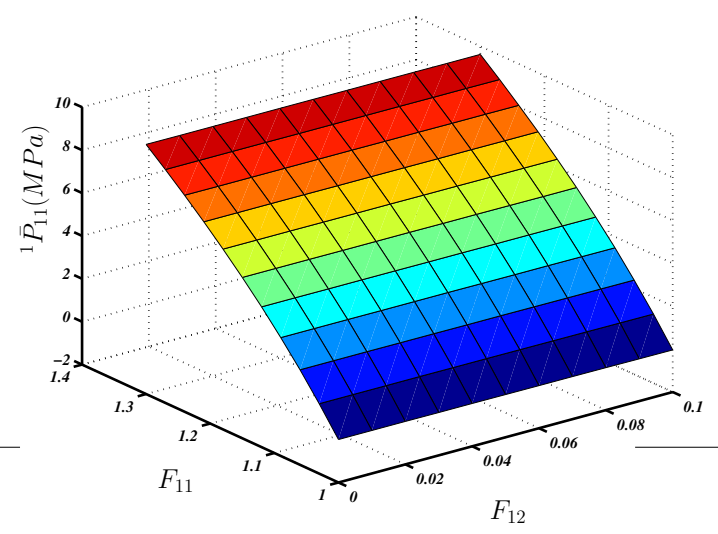

(c)

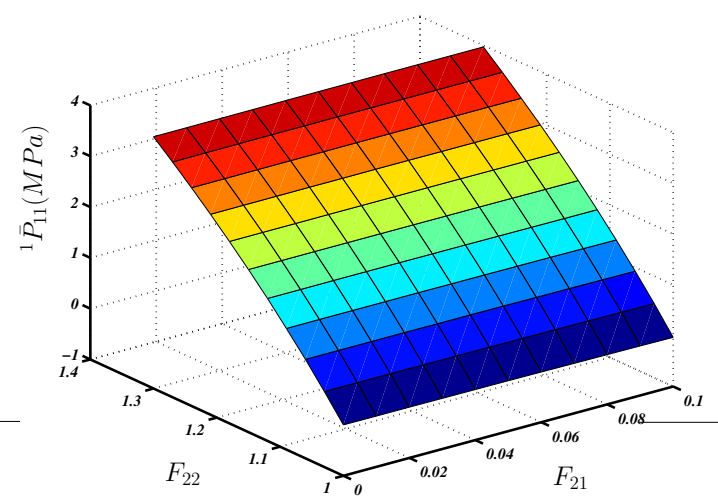

(e)

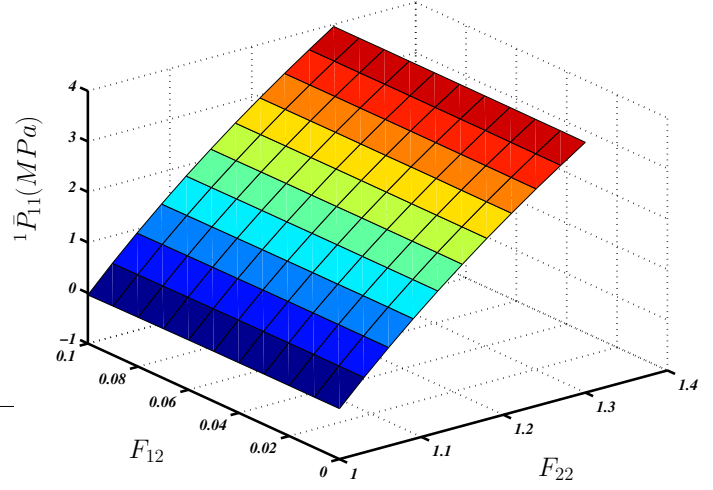

(b)

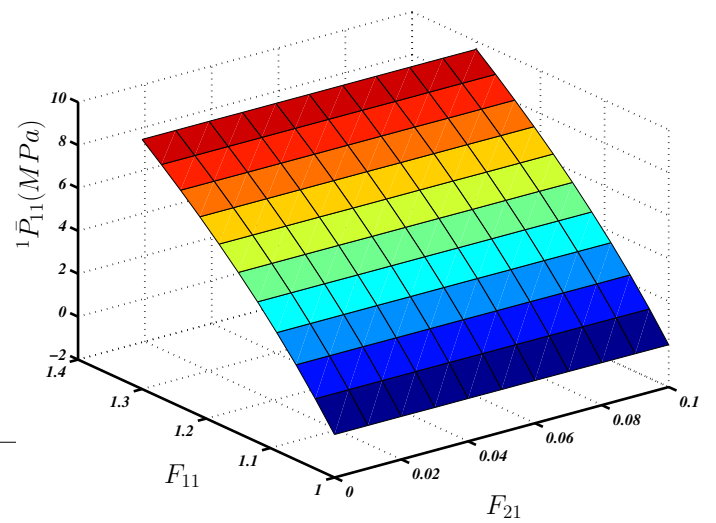

(d)

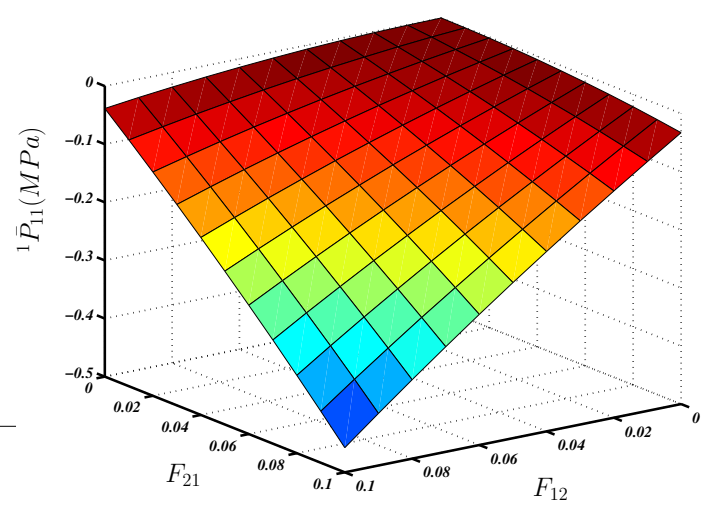

(f)

Figure 6.10: Simulated second order components of average microstress $\left({ }^{1} \bar{P}_{11}\right)$ with respect to the components of macroscopic deformation gradient tensor.

present study, three different deformation patterns that may arise at the macroscopic Gauss point are considered for the illustration purpose. 
First, a proportional type loading is considered where the components of $\overline{\mathbf{F}}$ as shown in Figure 6.11(a) increases in a proportionate manner as time proceeds. Figure 6.11(b) compares the components of average microscopic stress tensor with the full field finite element simulation.

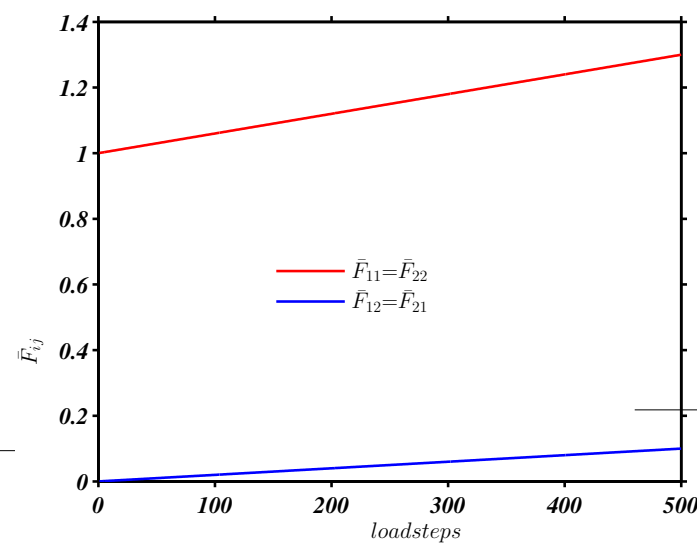

(a)

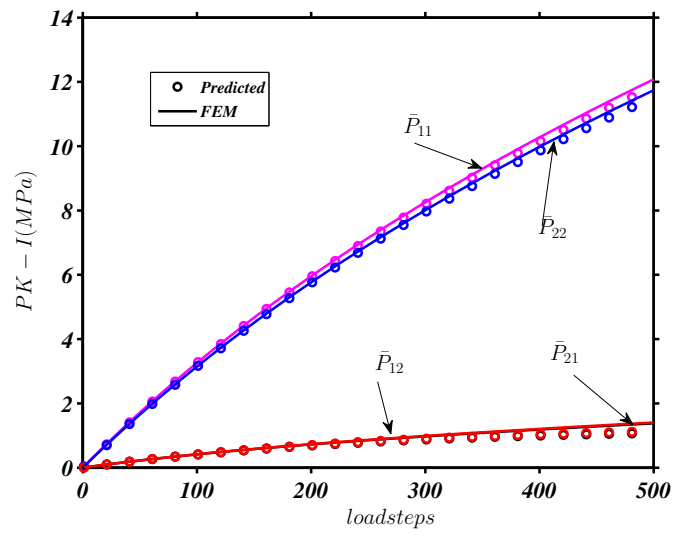

(b)

Figure 6.11: (a) Evolution of proportionally loaded macroscopic deformation gradient. (b) Comparison of predicted first Piola-Kirchhoff (PK-I) stresses with full field finite element simulation.

It can be found that the proposed scheme accurately predicted all the components of the average macroscopic stress tensor. Next, a nonproportional type loading is considered as as shown in Figure $6.12(\mathrm{a})$ where $\bar{F}_{22}$ and $\bar{F}_{21}$ increase linearly up to 500 load steps while $\bar{F}_{11}$ and $\bar{F}_{12}$ remain at the reference state (unloaded). At the next phase of loading (load steps $>500), \bar{F}_{22}$ and $\bar{F}_{21}$ remain constant at 1.2 and 0.08 , respectively, while $\bar{F}_{11}$ and $\bar{F}_{12}$ are varied linearly with load steps. For this loading scenario, it can be observed from Figure. 6.12(b) that the predicted components of the stress tensor follow their simulated counterparts almost exactly. Furthermore, a more complex loading situation consisting of loading-unloading of components of deformation gradient is considered (Figure. 6.13(a)). It can also be found from Figure. 6.13(b) that the predicted behaviors are similar to simulated responses. 


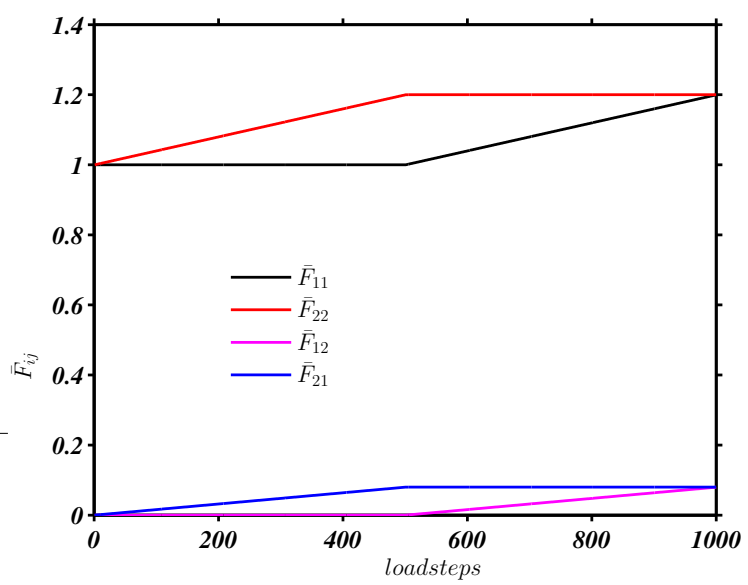

(a)

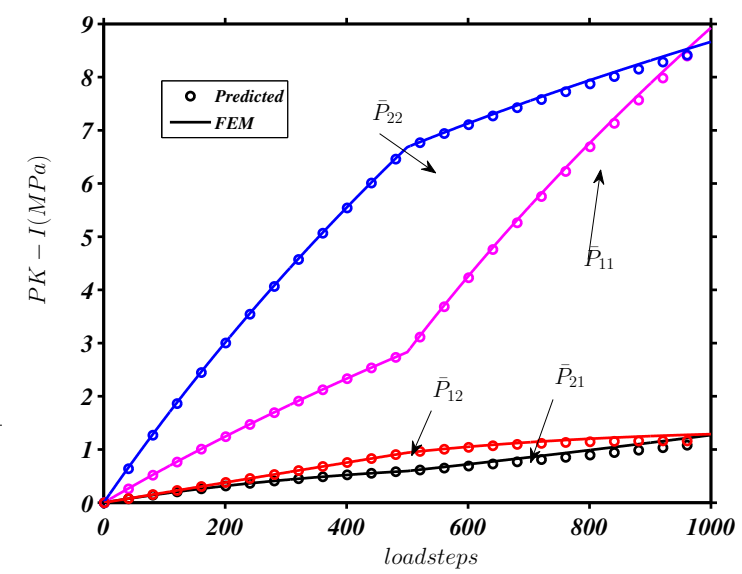

(b)

Figure 6.12: (a) Evolution of non-proportionally loaded macroscopic deformation gradient. (b) Comparison of predicted first Piola-Kirchhoff (PK-I) stresses with full field finite element simulation.

\subsubsection{Short fiber composite}

As a second example, a short fiber composite material similar is considered. The representative microstructure is given in the Figure. 6.14. The idea behind choosing such a RVE is to illustrate the effect of incorporation of material anisotropy on the prediction behavior by the proposed model. Similar to the particle composite, a compressible Neo-Hookean material is considered whose strain energy function is given in Equation (6.49). The material properties of the matrix is given as $\lambda^{(1)}=12.67$ 


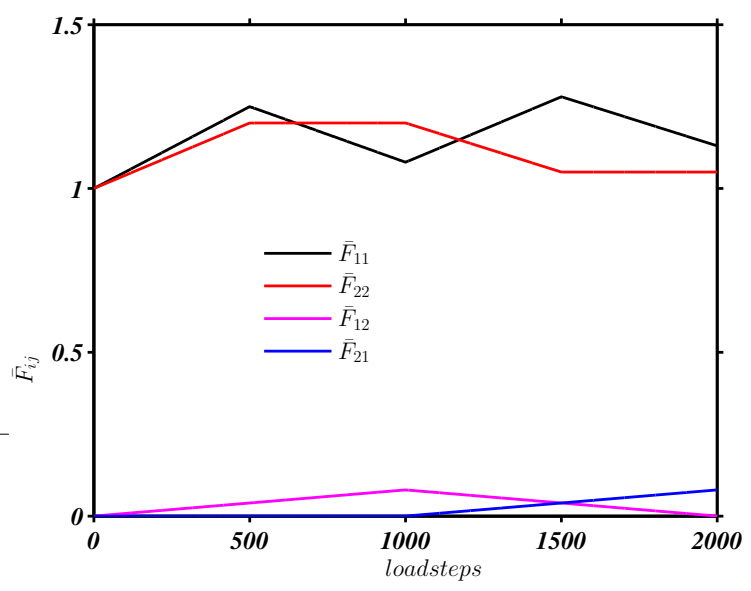

(a)

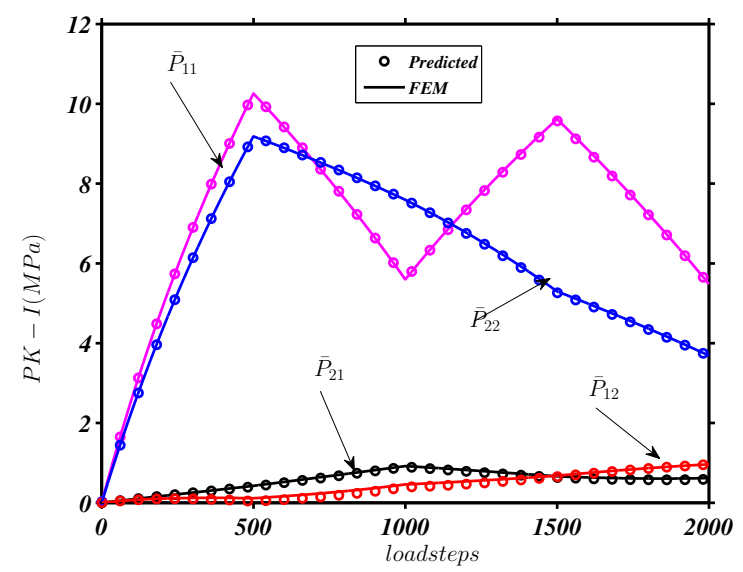

(b)

Figure 6.13: (a) Evolution of macroscopic deformation gradient with series of loading and unloading events. (b) Comparison of predicted first PiolaKirchhoff (PK-I) stresses with full field finite element simulation.

$M P a$ and $\mu^{(1)}=8.0 M P a$ whereas the fiber is considered highly stiffer and the material properties are given as $\lambda^{(2)}=500 \lambda^{(1)}$ and $\mu^{(2)}=500 \mu^{(1)}$. The similar magnitude of the maximum macroscopic deformation gradient tensor is also considered for this example. After constructing the surrogate model in a similar manner to the particulate composite, the average macroscopic stress is predicted. Three loading scenarios as given in Figure. 6.11(a), 6.12(a) and 6.13(a) are also considered for this RVE.

Predicted average microscopic stress for proportional type loading is compared with 


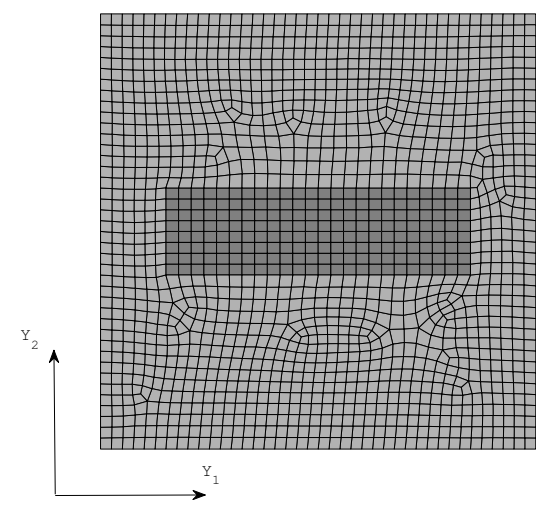

Figure 6.14: Representative volume element consisting softer matrix and short fiber. Fiber is denoted by darker regions, darker shade denoting higher stiffness.

full field finite element simulation in Figure 6.15(a). It can be found that, $\bar{P}_{11}$ and $\bar{P}_{22}$ are significantly different. Thus, insertion of short fiber imparts considerable anisotropy in the material response. However, predicted components of average microscopic tensors are in good agreement with the direct finite element simulation. Furthermore, the predicted behavior for the nonproportional type as well as the complex loading follow their simulated counterpart almost exactly.

\subsubsection{Three dimensional particulate composite}

To illustrate the efficiency of the proposed scheme in more complex material system, a three dimensional particulate composite is considered. Figure 6.16 shows a platelet embedded in a matrix of the composite. This particular geometry is considered to introduce anisotropy in the RVE. The compressible Neo-Hookean materials are considered for both matrix and platelet. The material properties of the matrix is given as $\lambda^{(1)}=12.67 \mathrm{MPa}$ and $\mu^{(1)}=8.0 \mathrm{MPa}$, whereas the platelet is considered stiffer and the material properties are given as $\lambda^{(2)}=200 \lambda^{(1)}$ and $\mu^{(2)}=200 \mu^{(1)}$. As mentioned earlier, the macroscopic deformation gradient tensor consists 9 independent components in three dimensions. Constructing the surrogate model with 9 input variable 


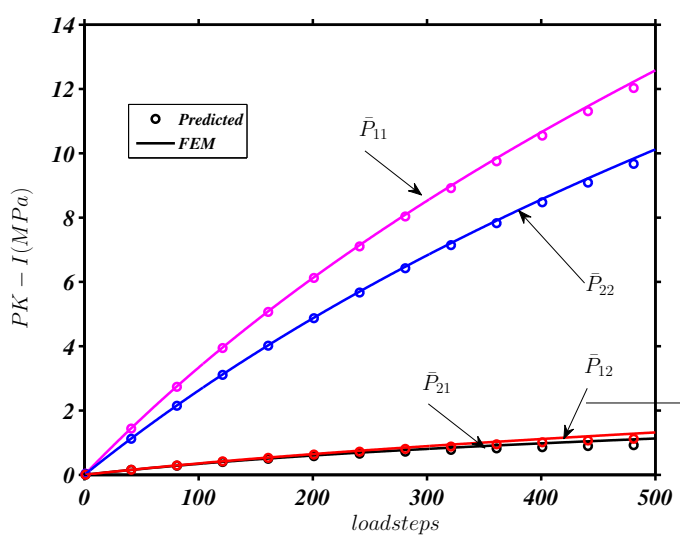

(a)

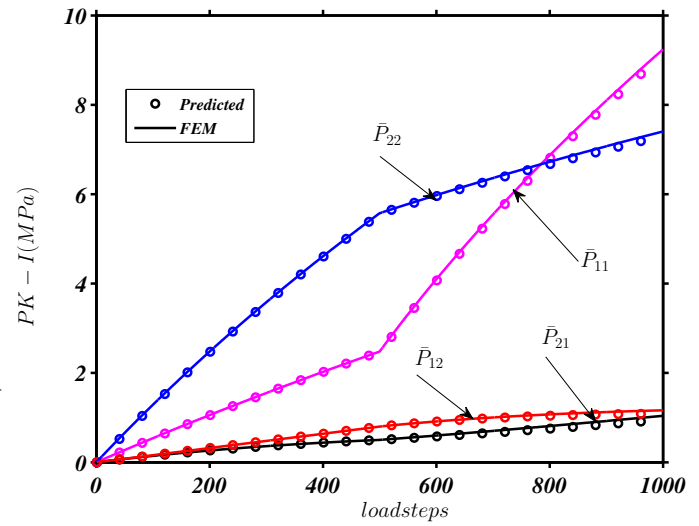

(b)

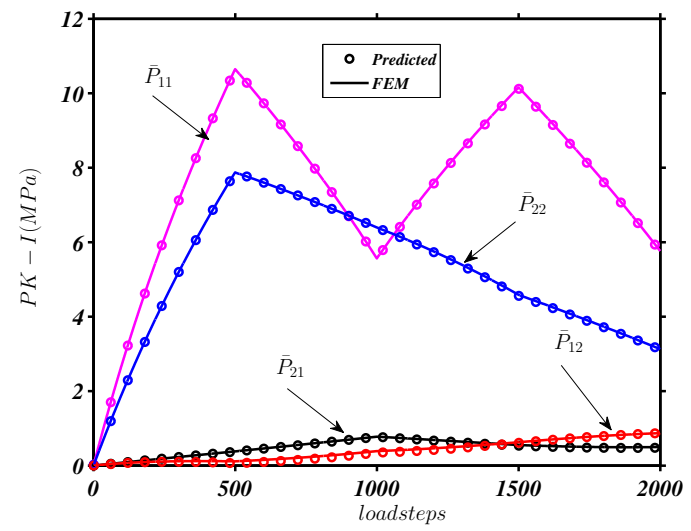

(c)

Figure 6.15: Comparison of predicted first Piola-Kirchhoff (PK-I) stresses with full field finite element simulation for (a) proportional loading, (b) nonproportional loading, and (c) complex loading-unloading.

is difficult. Hence, for the illustration purpose, we consider only 4 components of the macroscopic deformation gradient $\left(F_{11}, F_{22}, F_{33}\right.$, and $\left.F_{12}\right)$ offering a three dimensional loading situation. Similar to the previous examples, the surrogate component functions are generated assuming undeformed state as the reference state, and maximum deformation gradient as $\overline{\mathbf{F}}=\left\{\begin{array}{llll}\bar{F}_{11} & \bar{F}_{22} & \bar{F}_{33} & \bar{F}_{12}\end{array}\right\}=\left\{\begin{array}{lllll}1.3 & 1.3 & 1.3 & 0.1\end{array}\right\}$. The RVE is discretized with 10 noded tetrahedron, and homogeneous deformation gradient is applied as the boundary condition. 


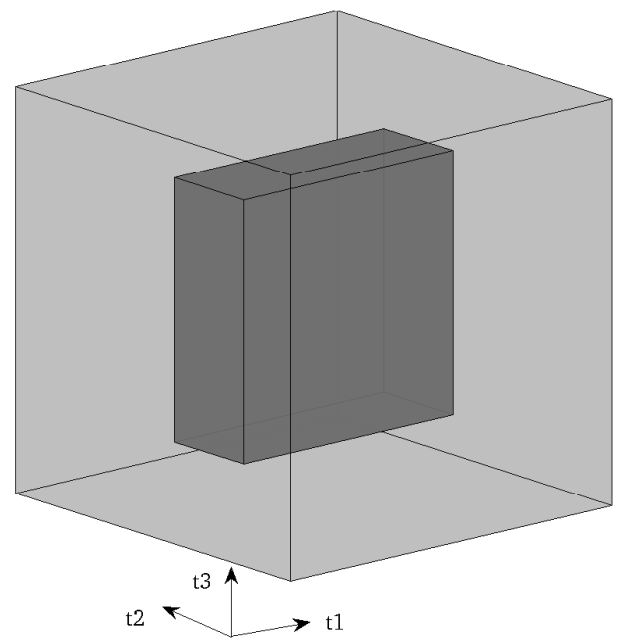

Figure 6.16: Three dimensional representative volume element consisting softer matrix and a platelet. Platelet is denoted by darker regions, darker shade denoting higher stiffness.

Initially, a proportional type load profile (Figure 6.17(a)) is considered for surrogate based prediction of the average macroscopic stress. Full field finite simulation is also performed along the load profile for the comparison purpose. It can be found from Figure 6.17(b) that the HDMR predicted response is similar to the actual finite element simulation. Similar to the previous example, the nonlinear hyperelastic composite is also subjected to a complex loading profile (Figure 6.18(a)). Predicted behavior of the composite and its simulated counterpart (Figure 6.18(b)) show a good agreement. Yvonnet et al. (2009) constructed a look-up table for the effective behavior of similar RVE made of compressible strain hardening material undergoing small deformation. Furthermore, they compared the predicted behavior obtained by direct interpolation with the full field finite element simulation. Construction of such lookup table requires $s^{3}$ sampling effort, i.e., 1331 finite element runs with 11 sampling points. The similar approach for large deformation analysis will require $s^{4}$, i.e., 14641 finite element simulations which is computationally expensive. Moreover, predicting the behavior for any arbitrary deformation gradient require higher dimensional interpolation which is also a difficult task. In contrast, the proposed method require only 


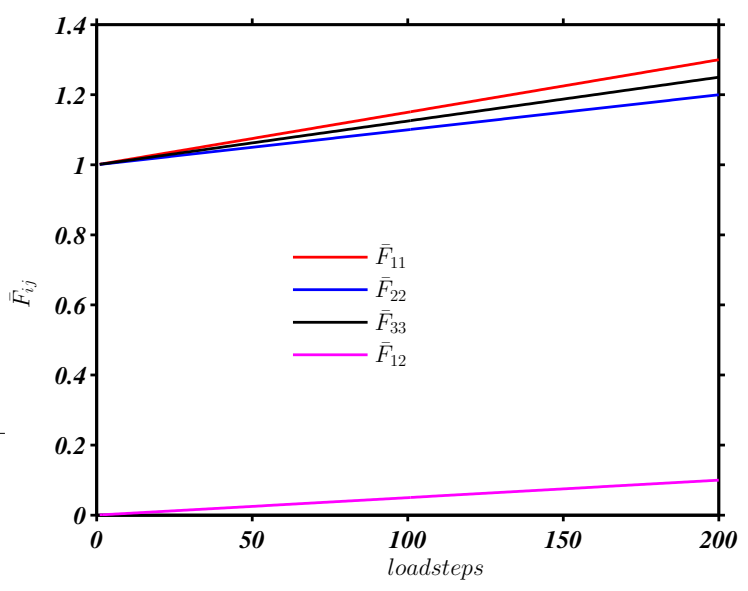

(a)

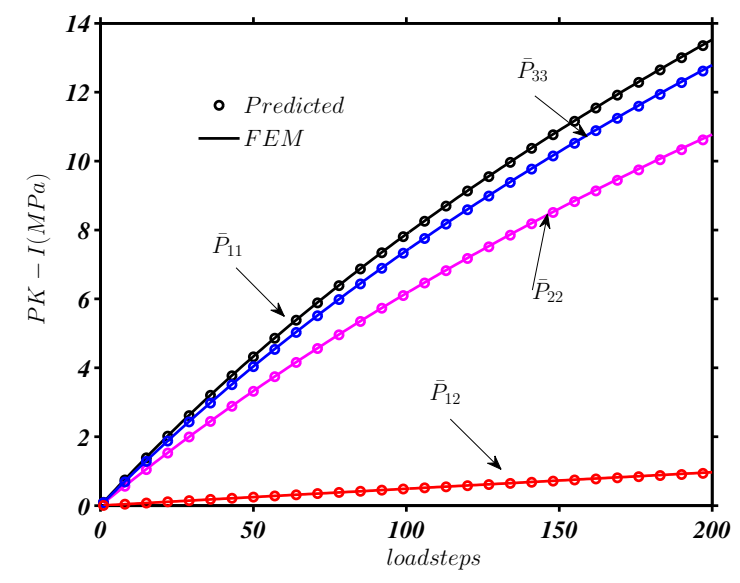

(b)

Figure 6.17: (a) Evolution of proportional type macroscopic deformation gradient. (b) Comparison of predicted first Piola-Kirchhoff (PK-I) stresses with full field finite element simulation.

641 finite element simulations for similar input sampling. Thus, the present scheme incur a significant amount of computational savings.

\subsubsection{Computational efficiency}

It should be noted here that the our predictions need not obey the temporal coherence. Once the surrogate model has been constructed, response prediction can be 


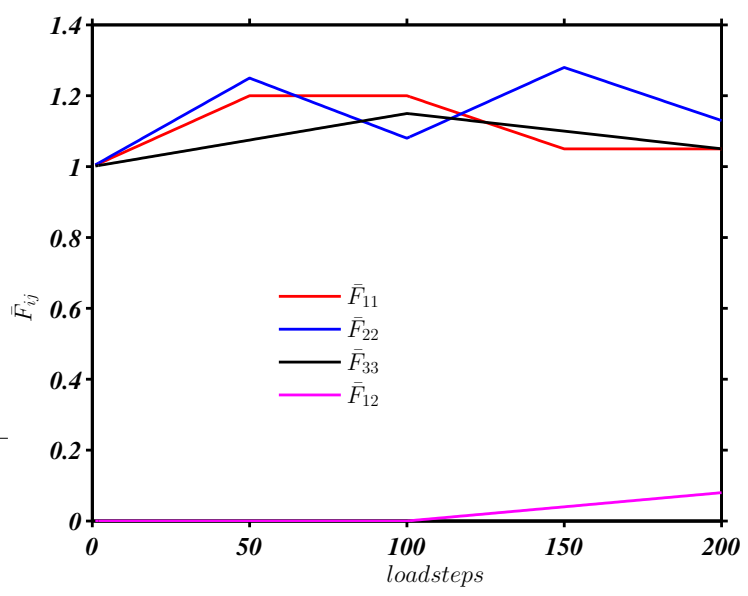

(a)

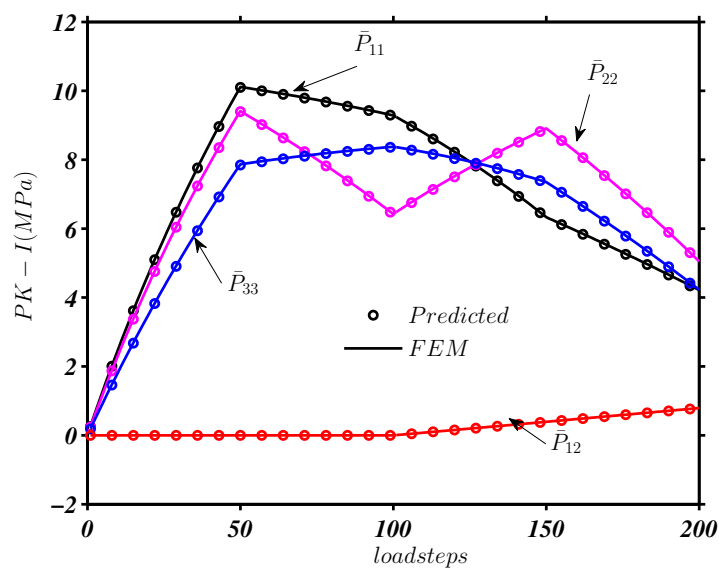

(b)

Figure 6.18: (a) Evolution of macroscopic deformation gradient with series of loading and unloading events. (b) Comparison of predicted first PiolaKirchhoff (PK-I) stresses with full field finite element simulation.

performed disregarding the evolution of the input deformation gradient. To indicate the computational efficiency of the proposed scheme, consider a macro-scale domain with $n_{e l}$ elements, $n_{g}$ Gauss points per element, $n_{l}$ load increments and $N_{I C}$ average number of macroscopic iterations. Let $t_{R V E}$ is the average time required to solve a RVE for each load step. Correspondingly, the total time to simulate the macroscopic $\mathrm{BVP}, t_{\text {Macro }} \sim n_{e l} n_{g} N_{I C} t_{R V E} n_{l}$. In the present study, $t_{R V E}$ for particulate composite, short fiber composite and 3D composite are given in Table 6.2. The construction of 
surrogate model requires solving of $N_{f}$ microscopic BVP problem with $n_{s}$ load steps which offers $t_{\text {surr }} \sim N_{f} n_{s} t_{R V E}$. This is the most costly step e.g., particulate composite offers $t_{\text {surr }}=534.16$ cpu hrs. However, finite element simulations required for surrogate model are independent from each other and can be performed over massively parallel computers. As the HDMR prediction is based on low-dimensional interpolation, it takes negligible amount of time which is order of $\mu s$ (see $t_{H D M R}$ in Table 6.2). Therefore, the computational efficiency of the proposed scheme can be defined as

$$
\eta=\left(1-\frac{t_{\text {surr }}}{t_{\text {Macro }}}\right) \sim 1-\frac{N_{f} n_{s}}{n_{e l} n_{g} N_{I C} n_{l}}
$$

It can be seen from the above equation that the proposed method is particularly advantageous when, in a large structure, a large number of similar RVEs are experiencing dissimilar deformation history. In the proposed scheme, stress predictions can be performed taking into account only the current combination of the components of the deformation gradient tensor. Thus, material response at any point on macroscale is readily available. Moreover, it requires only simulated zeroth, first and second order components of stress tensor. Thus, costly storage and retrieval of previous states is completely eliminated in our method.

Table 6.2: Comparison of computational cost for each load step

\begin{tabular}{|c|c|c|}
\hline \hline Type of composites & HDMR Prediction $\left(t_{H D M R}\right)$ & FEM simulation $\left(t_{R V E}\right)$ \\
\hline Particulate & $52 \mu \mathrm{s}$ & $5.96 \mathrm{~s}$ \\
Short fiber & $52 \mu \mathrm{s}$ & $6.34 \mathrm{~s}$ \\
3D composite & $90 \mu \mathrm{s}$ & $48.00 \mathrm{~s}$ \\
\hline
\end{tabular}




\subsection{Concluding remarks}

In this chapter, we have demonstrate the applicability of Higher Dimensional Model Representation (HDMR) based surrogate model to reduce computational burden. Construction of failure envelop of heterogeneous material and two scale finite element method have been considered as illustrative examples. First, a surrogate model based feasibility formulation has been developed and applied to construct the failure envelop of heterogeneous microstructure. Component functions of the surrogate model are generated using the Cut-HDMR technique which requires significantly reduced number of finite element simulations. To test the accuracy, direct finite element simulations have also been performed to determine the failure envelop. As the second example, a two scale finite element framework for heterogeneous material with nonlinear hyperelastic constituents undergoing finite strain has been choosen. The surrogate model has been applied to replace the lower scale simulations. After constructing the surrogate models for different RVEs, the average response is predicted for arbitrary deformation history and is compared with full field finite element simulations. The following conclusions can be drawn from two applications:

- The HDMR based surrogate model can accurately capture the range of applied input strain parameters over which the design retains feasibility. Thus, the proposed methodology can have promising application in predicting the failure envelop of complex materials systems.

- Accuracy of the proposed 2nd order HDMR approach is very high as shown by various examples. Complex nonproportional loading scenarios for a 3D highly nonlinear RVE yielded excellent match to the full field finite element simulation results at a fraction of the original computational cost. Thus, for two scale finite element, lower scale simulation can be substituted with the HDMR based 
surrogate model. Hence, concurrent multiscale simulation can be expedited significantly.

- The surrogate model requires only the component functions (zeroth, first and second order) to be stored which needs less amount of data storage. 


\section{Chapter 7}

\section{Conclusions and future work}

\subsection{Salient findings of the present work}

Heterogeneous materials possess significant challenges in characterizing their mechanical behavior. In the present study, we mainly focused on two types of heterogeneous materials: cellular materials and mussel byssus threads. Understanding mechanical behavior of cellular materials is extremely difficult during impact loading. However, an impact loading scenario is one of the most common operative environments for this class of materials. Although the split Hopkinson pressure bar (SHPB) experiment provides the dynamic response of a wide range of materials, it is difficult to extract the dynamic response for soft cellular materials. SHPB testing of cellular materials can be performed only through suitable modifications which require prior knowledge of the stress-state within the specimen. However, such detailed knowledge of stress-state with respect to cellular microstructural and experimental parameters is still lacking. Moreover, high strain rate energy absorption characteristics of cellular materials depend on a number of intertwined microscopic mechanisms (microinertia, 
microbuckling and microbending). Present literature lacks a complete description of the complex interactions of these mechanisms arising at the microstructural level.

We have also studied a second type of heterogeneous material: Mussel byssus thread. The design of these thread is the demonstration of intelligent use of heterogeneities at the microstructural level to achieve superior mechanical performance. Experimental observations offer the mechanical properties of mussel byssus threads and their microscopic origin. However, contribution of macroscopic heterogeneity in-terms of proximal and distal thread lengths towards its strength, toughness and extensibility has never been addressed. Such knowledge is required to exploit its potential towards development of biomimetic tough thread.

Furthermore, analysis of complex materials in several situations involves computational burden. For example, construction of failure envelope of a heterogeneous material requires failure criteria to be tested over entire input loading parameter space. Thus, it involves large number of finite element simulations. Similarly, in two scale finite element method, a representative volume element of heterogeneous materials needs to be solved for average mechanical response at the macroscopic point. Such concurrent simulations are expensive. Thus, computationally efficient predictive models are highly desirable in these situations.

To investigate the deformation characteristics of soft cellular solids and mussel byssus threads, a robust numerical framework has been developed in this thesis. An interconnected network structure has been considered for cellular solids, while a filament type model has been assumed for mussel byssus thread. To simulate the mechanical behavior for both the situations, a corotational beam formulation that can capture the finite deformation kinematics has been adopted. A modified corotational beam formulation has been proposed to evaluate the rotation quantities precisely. Based on the modified beam approach and a central difference scheme, an explicit dynamic 
finite element framework has been developed to simulate dynamic response of cellular materials. Furthermore, an implicit quasi-static corotational beam finite element formulation has been developed to simulate the mechanical behavior of mussel byssus thread. Based on the principle of Voronoi tessellation, a wide variety of cellular microstructures have been generated. To simulate the cellular microstructures, the struts have been discretized with two noded Timoshenko beam elements. The explicit dynamic finite element framework has been used to enumerate the microscopic mechanisms responsible for the macroscopic response of cellular materials. Furthermore, with the application of suitable boundary conditions, the stress-state of cellular specimens has been investigated during SHPB tests.

To reveal the intricacies involved in the smart design of mussel byssus thread, a Genetic Algorithm (GA) based optimization framework has been developed. The primary interest is to find the optimal distribution of mussel byssus thread components to achieve maximum toughness. The implicit quasi-static finite element formulation that can be used to find the toughness of byssus thread for a given set of geometric and material properties has been integrated within GA framework.

Finally, to reduce the computational complexity arising in analysis of heterogeneous materials, the surrogate modeling concept has been proposed. Such surrogate models are constructed based on Higher Dimensional Model Representation (HDMR) which requires a fewer number of numerical simulations. This methodology has been applied in construction of the failure envelope of a highly heterogeneous material. Furthermore, HDMR based surrogate methodology has also been implemented in multiscale framework to simulate nonlinear heterogeneous materials undergoing finite strain and nonproportional loading. From the present work, the following accomplishments have been achieved;

- Stress-state in cellular specimen during SHPB testing greatly depends on its 
intrinsic and extrinsic parameters. Larger cell size cellular specimens mostly offer nonequilibrium stress-states. The average number of cells in the specimen length does not affect the stress state but does affect the converged response. This analysis suggests that cellular specimen must contain large number of cells (>16) along the length direction to exhibit the representative dynamic response. Equilibrated stress state can not be achieved with higher loading rates. Higher impedance mismatch has been suggested for lower relative density cellular specimen. Therefore, such in-depth understanding of the stress-state will facilitate the experimentalist to incorporate suitable modifications to accurately obtain the constitutive response of soft cellular specimens.

- Present investigation also provides a thorough understanding of micromechanisms responsible behind the origin of macroscopic behavior of open cell soft cellular material under dynamic loading environments. It is observed that microinertia followed by microbuckling are responsible for early stress enhancement, and rapid drop in macroscopic stress strain response. These two micromechanisms also offer progressive collapse of cellular materials. In the absence of microinertial enhancement, microbending of the struts dominate resulting in uniform collapse of the cellular microstructures. These three micromechanisms behind the origin of macroscopic stress strain response and collapse patterns greatly depend on cell size, bulk material properties, cell irregularities, cell orientation and loading rates. Therefore, the knowledge of deformation characteristic of cellular materials will assist in designing cellular microstructure precisely to meet specific performance requirements.

- Similar to other tough biological materials, material heterogeneity also plays a significant role in achieving the toughness of mussel byssus thread. Present investigation on mussel byssus thread exhibits that different types of collagens are optimally distributed along the thread to accomplish maximum toughness 
along with high extensibility. Such optimal distribution highly depends on the ratio of failure strength to the Young's modulus of the proximal thread and ratio of Young's modulus of distal thread to proximal thread. However, optimal distribution remains unaffected by the higher value of the ratio of Young's modulus of distal thread to proximal thread. Therefore, this analysis will provide a suitable pathway to develop biomimetic tough threads.

- HDMR methodology based surrogate models are highly efficient in predicting the behavior of complex material systems. The failure envelope for a particulate composite can be achieved with significantly lesser computational burden. It is also found that the surrogate model can predict the mechanical response of representative volume elements with nonlinear hyperelastic constituents undergoing finite strain. Thus, in the two scale finite element technique, lower scale can be replaced by surrogate model. Therefore, a significant amount of the computational burden can be reduced. Hence, HDMR methodology can be

pursued as alternative strategy for constructing surrogate involving significantly lesser computational efforts.

\section{2 $\quad$ Future directions}

The explicit dynamic corotational framework along with cellular microstructure generation methodology provided an in-depth understanding of the micromechanisms associated with macroscopic response of soft cellular materials. The structure-property relationships under dynamic loading as found in the present analysis are of utmost importance for designing cellular microstructures. However, the effect of microstructural complexities need to be further investigated towards effective design of cellular solids. The present framework will assist to understand the mechanical response of 
a wide variety cellular specimens made of metals as well as ceramics. Such extensions can easily be incorporated by using different constitutive material models in the corotational beam formulation. Furthermore, the numerical framework can be used to fabricate defect tolerant cellular microstructures through analyzing the collapse behavior of foams with microstrcutural defects (fractured cell wall, waviness of strut and large holes). The effect of cellular microstructural anisotropy (elongated cell) can also be predicted. The present framework will also facilitate in designing graded and layered cellular microstructures towards ballistic applications. Moreover, several biological materials exhibit cellular type architecture containing biological fluids. Incorporation of viscous effect within the present framework will allow us to investigate the mechanical response of biological materials more precisely.

The Genetic Algorithm based optimization framework integrated with mechanical model of byssus thread established that material heterogeneities in the threads are optimally distributed to achieve maximum toughness. Though demonstrated on a relatively simple system, such methodology can be used to design complex material systems with specific performance requirements. By suitably defining the performance criterion (objective functions) and replacing the mechanical model of byssus thread with a complex numerical model (nonlinear finite element framework), the GA based optimization framework will serve as complex microstructure design tool.

The HDMR based surrogate models illustrated that mechanical response of complex nonlinear heterogeneous material can be predicted accurately over an arbitrary input loading profile at the fraction of the computational cost full set of simulations. Thus, the HDMR based surrogate model can offer broader impact in the areas of computational material design. Although several particulate composites with nonlinear hyperelastic constituents have been taken as representative volume elements in the present study, replacement of such material systems with cellular type materials can be performed. As found earlier, cellular materials offer localized deformation. Therefore, 
applicability of surrogate model in predicting the dynamic response of cellular materials needs to be investigated. Besides, predictions of macroscopic response history for any arbitrary set of input parameters requires surrogate model to be generated at each time steps. The HDMR based surrogate model can be extended to find the sensitivity of the macroscopic behavior of the material system on its input parameters. Thus, these surrogate models along with sensitivity information of microstructural components will provide cost effective design of heterogeneous materials. Moreover, it can be conjectured that the optimization framework for complex materials such as GA is computationally intensive because objective function evaluation will typically involve multiple finite element simulations. HDMR based surrogate models can substitute for the costly numerical simulations. Therefore, complex microstructure design can be possible with reduced computational burden. Furthermore, surrogate model can play significant role in the area of inverse parameter estimation. During inverse parameter estimation, the response of mechanical system must be evaluated. For a complex material system, numerical models must be simulated for arbitrary set of system parameters. Such numerical simulations can be substituted with the HDMR based surrogate model. Thus, HDMR based surrogate model will play significant role in reduction of computational complexity and will open a new paradigm in designing heterogeneous materials. 


\section{Appendix A}

\section{Cell level analysis of microinertia and microbuckling in soft cellular materials}

\section{A.1 Introduction}

Enhancement in peak stress with an increase of the rate of applied loading is especially severe for low strength and impedance materials (Song et al., 2007). Such peak stress must be lower than the failure stress of the supporting structure for fail safe design. Thus, understanding of stress enhancement mechanisms is a key for designing components during impact loading scenario. The rate sensitivity of a material can arise from two sources: the material properties itself can be rate dependent, or it can arise from the structural response of the dynamically loaded volume (Gary and Bailly, 1998). For bulk materials, the second source of rate sensitivity is variously explained to be arising from lateral inertia of the body (Forrestal et al., 2007; Song et al., 
2007; Gary and Bailly, 1998), Poisson effect (Chen and Ravichandran, 2000), confining pressure (Li and Meng, 2003; Nittur et al., 2008), or the nucleation and propagation of microcracks in the material (Klepaczko, 1988). However, for a cellular architecture, collective structural dynamic behavior of struts and/or cell walls determines the macroscopic materials response. A complex interplay among microinertia, microbending and microbuckling at the cell level has been envisaged as the contributing factor towards the overall dynamic behavior in this class of materials. These intertwined phenomena greatly depend on foam topology, material properties of the bulk material and imperfections in the microstructure, and it is often difficult to study the contribution of individual mechanisms on the overall material behavior. Zhao et al. (2006) reviewed the possible reasons for the macroscopic strength enhancement of cellular material which comprise of strain rate sensitivity of the bulk material. Effect of pressure of the gaseous medium entrapped in the cell in case of closed cell foam (Bouix et al., 2009), the microinertia of the cell wall (Zhao et al., 2005) and shock enhancement (Tan et al., 2005; Reid and Peng, 1997; Harrigan et al., 1999) are put forward as possible peak stress enhancement mechanisms.

While the bending and buckling phenomena at the cell level have enjoyed considerable attention in the study of quasi-static as well as dynamic behavior of cellular solids (Gibson and Ashby, 1997; Rosakis et al., 1993; Vural and Ravichandran, 2003; Zhao et al., 2005; Ruan et al., 2003), the effect of microinertia on the peak stress enhancement has not been discussed in great detail. However, recent experimental and numerical studies show that inertia can exhibit a substantial effect especially for low strength and impedance materials (Song et al., 2007; Pal et al., 2010). Analytical models as well as experimental observations are available in literature to analyze two types of plastically deforming structures under impact loading. Type I structure (transverse compression of circular tubes) having quite flat topped force displacement after yield point presents limited rate sensitivity. On 
the other hand Type II structure (buckling of straight beam) shows a sharp drop of crushing force after a critical loading point and significant rate sensitivity is observed (Calladine and English, 1984). The rate sensitivity characteristics of Type II structure drew considerable attention from the researchers to study the inherent mechanism theoretically or numerically (Su et al., 1995; Langseth and Hopperstad, 1996; Langseth et al., 1999; Karagiozova and Jones, 1996; Gao et al., 2005) and their result showed that lateral inertia contribute significant role in dynamic response for type II structure. Vural and Ravichandran (2003) presented a simplified model (similar to Type II) to understand and quantify the importance of microinertia on experimentally observed dynamic strength enhancement. In their analysis the effect of inertia was analyzed by modeling the buckling instability similar to the model developed by Tam and Calladine (1991) where two axially deformable rods freely hinged each other at connecting node subjected to a dynamic loading from one end. In order to address the microinertia induced strength enhancement of cellular material, Zhao and Abdennadher (2004) studied the crushing test of square tube of rate insensitive material under dynamic loading conditions. Similarly, Romero et al. (2008) developed a micromechanical model to understand the inertial and viscous strain rate effect on the behavior of dynamically loaded cellular material. All above modeling studies show that the microinertial enhancement originates from the motion of cell wall during dynamic loading which depends on cell wall properties and cell topologies. Although these studies provide a physical understanding of the origin of such enhancement, it has not been rigorously analyzed for different cellular material and microstructural properties. Furthermore, softening behavior of stress strain response after the inertial enhancement as well as the subsequent oscillations as found in several experimental observations (Zhao et al., 2005; Lee et al., 2006; Thomas et al., 2004) has never been addressed in great detail. Such an analysis is important in order to understand the contribution of cellular design parameters on the macroscopic rate sensitivity which stems from lateral inertial effect and associated 
mechanisms at cell level. The present study aims towards an in-depth understanding of the micromechanisms to address the dynamic response and associated deformation evolution of cellular material for different microstructural and material parameters at a wide range of loading scenario.

In the current study, we develop a mechanical model that will simulate collapse of a single cell in a dynamic loading environment. The model is based on the cell level deformation information of cellular solids obtained from a detailed micromechanical numerical analysis described elsewhere (Pal et al., 2010). We have considered an open cell configuration for the microstructure of the material. Bending of individual struts has not been taken into account, and the base material of the cellular specimen is considered to be rate insensitive. However, the model can simulate inertial as well as buckling response of the struts, and the transition of deformation regime governed by microinertia to that by microbuckling. Of special interest is the effect of various geometric and material properties on the inertial enhancement of stress along with softening response for these materials.

This chapter is organized as follows: the details of analytical formulations are presented in Section A.2. In Section A.3, a thorough discussion of the parametric study is illustrated. Of special interest is the effect of impact velocity, cell size (or strut length), bulk material properties, randomness in cell size and material properties on the deformation response at cell level. Finally, we end our discussion by drawing conclusions from the present study in Section A.4. 


\section{A.2 Analytical model}

An analytical model that represents collapse of a single cell has been developed to explain the effect of microinertia on the mechanical response of soft cellular specimens subjected to different microstructural, material and loading parameters. The model is inspired from the dynamic deformation behavior of a single cell. Fig. A.1(a) shows deformation of a foam specimen from the finite element simulation of polyethylene foam when subjected to an impact load (Pal et al., 2010). The magnified view of a particular cell is shown for both undeformed as well as deformed configuration. We are particularly interested in capturing the micromechanisms involved in the strut deformation, and consequently the displacement history of one half of a strut (segment ' $a$ ') is analyzed here. In this figure, the segment ' $a$ ' is connected to two other segments designated by ' $b$ ' and 'c'. The individual normalized lateral displacements $\left(d_{1} / l\right)$ of points $\mathrm{P}$ and $\mathrm{Q}$ as well as their normalized relative displacement are illustrated in the first figure in Fig. A.1(b). It can be observed that, the lateral displacements of $\mathrm{P}$ and $\mathrm{Q}$ undergo two distinct phases (as indicated by dotted line). In the first phase, both $\mathrm{P}$ and $\mathrm{Q}$ show negligible lateral displacement signifying a resistance on lateral motion. However, in the second phase, Q moves substantially in the lateral direction while the point $\mathrm{P}$ shows almost a negligible movement. Thus, $\mathrm{P}$ remains almost in the vertical plane during collapse of the cell. The normalized relative displacement of points $\mathrm{P}$ and $\mathrm{Q}$ in the vertical direction is also depicted in second figure in Fig. A.1(b). The vertical displacement history also offers two different phases of deformation as indicated by the dashed line. Furthermore, it can be noticed that at very beginning of first phase (indicated by dotted line), the normalized relative displacement of point $\mathrm{P}$ with respect to $\mathrm{Q}$ i.e., $\left(d_{2 P^{-}} d_{2 Q}\right) / l$ is almost zero which suggests a rigid body translation of the segment ' $a$ ' in vertical direction. Next, the

point $\mathrm{P}$ starts to move slightly with respect to $\mathrm{Q}$ which can be assumed that the segment 'a' undergoes axial compression $\left(d_{2 P}-d_{2 Q}=0.01 l\right)$ in the first phase. In the 
second phase, $d_{2 P^{-}} d_{2 Q}$ increases rapidly and becomes $O(l)$. This indicates that $\mathrm{P}$ reaches almost to the same level of $\mathrm{Q}$. The above observation clearly suggests that segment 'a' undergoes a transition from a vertical to a horizontal configuration during the collapse evolution. Thus, it can be concluded that beside an initial rigid body movement in vertical direction, the strut exhibits axial deformation and finally a rotation to accommodate the applied displacement. Moreover, microbending of the strut may be other mechanisms responsible for observed deformation characteristics. We envisage that high strain rate experiment on such an isolated cell wall will reveal the microscopic origin of the macroscopic stress enhancement mechanisms. Hence, to understand the micromechanisms involved in the cell level we develop the following model.

In the present analysis, we disregard the initial rigid body motion as well as microbending of the strut. These assumptions are reasonable as we are primarily interested in the effect of microinertia on the overall stress-strain response. Thus, we restrict our analysis to the interaction of microinertia (represented by axial shortening) and microbuckling (represented by rotation). The deformation of the strut is assumed to consist of Phase I associated with mainly axial deformation of the strut, whereas in phase II, rotation of the strut occurs. Therefore, the applied displacement is considered to be accommodated by axial shortening as well as the rotation of the strut. The dynamic deformation of strut ' $a$ ' is considered, whereas struts 'b' and 'c' are replaced by an equivalent spring that restricts the lateral motion (Fig. A.2 left). The half length of the strut ' $a$ ' is $l$ and $\theta_{0}$ is the initial misalignment angle while in Fig. A.2 (left) the current angle is $\theta$ and the overall length is $l-u$ (Fig. A.2 right). The axial shortening of the strut $u$ occurs due to applied vertical velocity at point P. As we are modeling the deformation of elastomeric cellular specimen; hence, no plastic deformation arises. Mass of the strut $m\left(=\rho_{s} A l\right)$ is lumped at the hinge 'Q' and $k$ represents the stiffness of the spring. Microbending of the strut ' $a$ ' is neglected here 


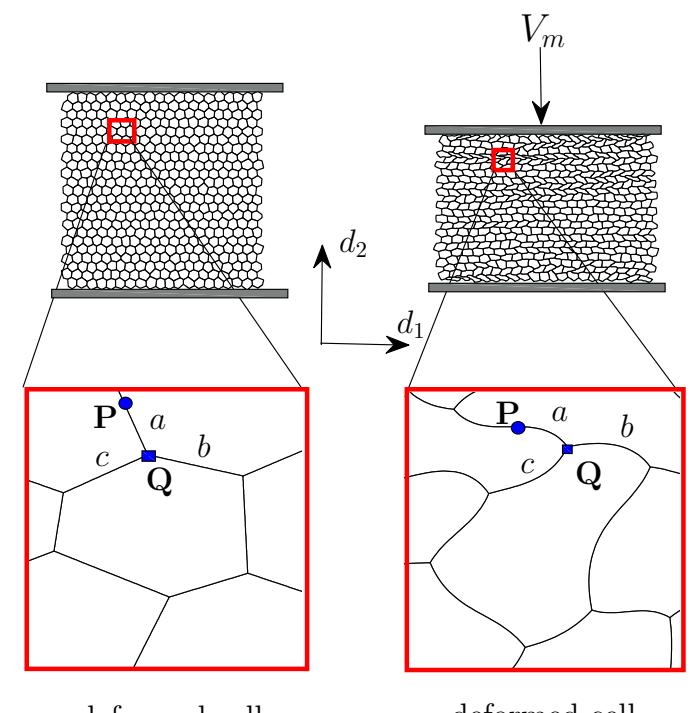

undeformed cell

deformed cell

(a)
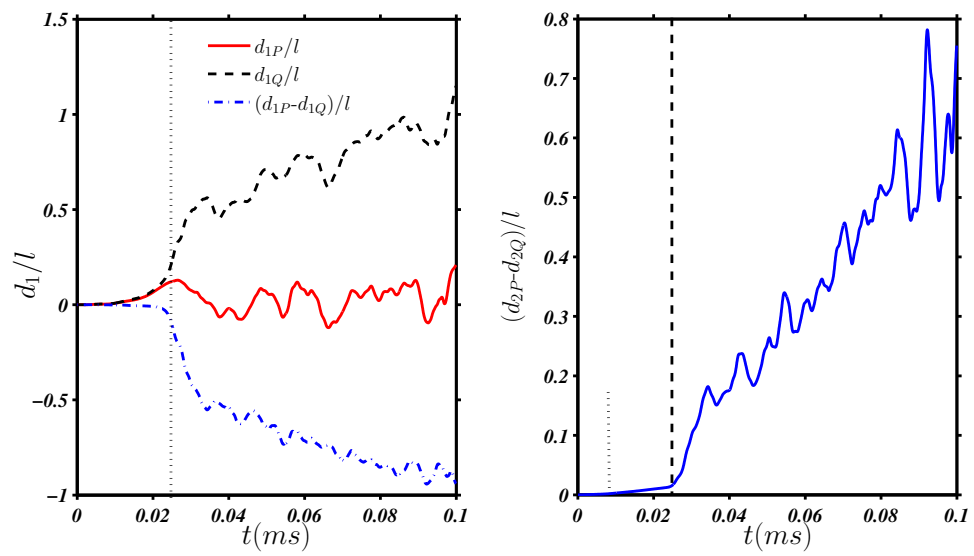

(b)

Figure A.1: (a) Magnified view of undeformed and deformed cell of a cellular specimen made of polyethylene when subject to an impact load from top (Pal et al., 2010). (b) Left figure shows normalized lateral displacement history of end points of segment 'a'. Right figure depicts the normalized relative vertical displacement history of point $\mathrm{P}$ with respect to $\mathrm{Q}$ of the strut segment. 

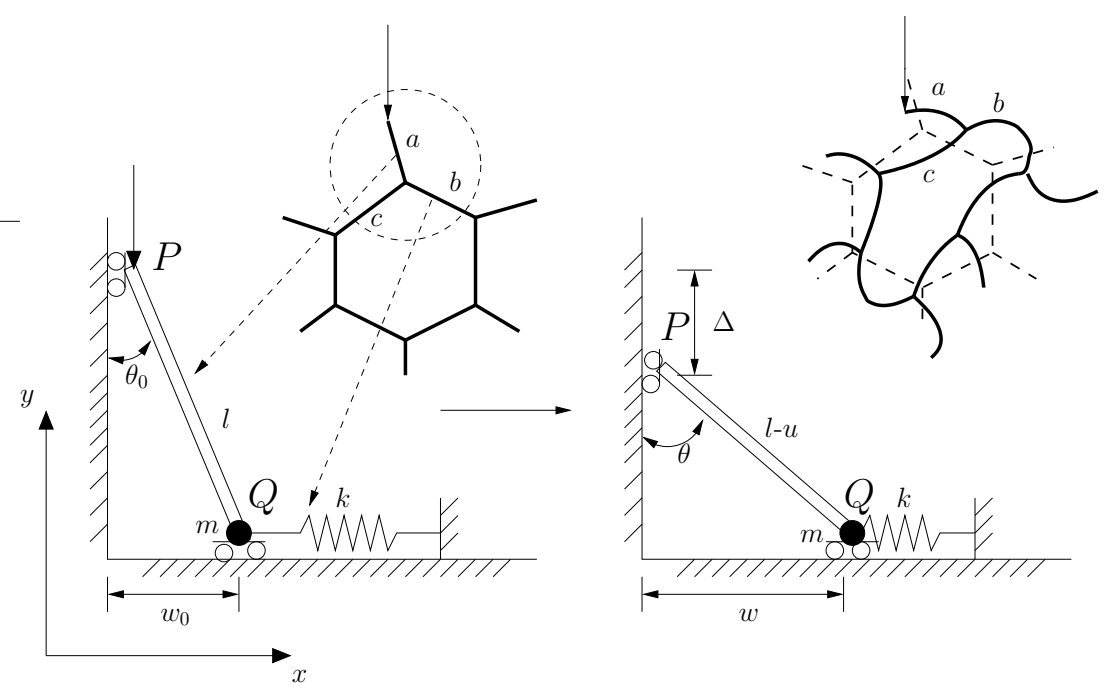

Figure A.2: Schematic representation of deformation of strut when subjected to a vertical impact velocity $v$.

for mathematical simplicity. The axial shortening of the bar gives rise to microinertial effect, whereas rigid body rotation of the bar ' $a$ ' is considered as microbuckling.

The reduced vertical length can be computed geometrically from Fig. A.2 as

$$
\Delta=l \cos \theta_{0}-(l-u) \cos \theta .
$$

The transverse displacement $w$ and current angle $\theta$ can be related as

$$
\cos \theta=\frac{\sqrt{l^{2}-w^{2}}}{l} .
$$

Substituting the above relation in Equation (A.1), the axial displacement of the strut $u$ can be found as

$$
u=\frac{l}{\sqrt{l^{2}-w^{2}}}\left(\Delta-l \cos \theta_{0}\right)+l .
$$

From the above equation, the transverse displacement can be expressed as function of axial shortening $u$ and reduced vertical length $\Delta$ as

$$
w=f(u, \Delta)=\left[l^{2}-\frac{l^{2}}{(l-u)^{2}}\left(l \cos \theta_{0}-\Delta\right)^{2}\right]^{1 / 2} .
$$


In the above equation $\Delta$ depends on the type of applied displacement. For a constant vertical shortening rate $\dot{\Delta}=v$ gives $\Delta=v t$ for $\Delta=0$ at $t=0$, whereas a linear velocity $\dot{\Delta}=C t$ which results $\Delta=C t^{2} / 2$. The axial force $F$ of the strut arising from the axial shortening $u$ offers a lateral force on the mass. Correspondingly, governing equation for lateral motion of the strut can be expressed in terms of the axial force and the resistance offered by the spring as

$$
m \ddot{w}=F \sin \theta-k\left(w-w_{0}\right) .
$$

As we are interested in elastomeric cellular materials, a linear elastic constitutive material law has been assumed as $\sigma=E \varepsilon$ and consequently, the axial force of the strut can be found as $F=\sigma A=E A u / l$. The spring stiffness $k$ is considered as the equivalent stiffness of the bars ' $b$ ' and 'c' in Fig. A.2 for horizontal displacement of point 'Q' and can be expressed as

$$
k=\xi_{1} \frac{E A}{2 l} f_{1}(\phi)+\xi_{2} \frac{12 E I}{(2 l)^{3}} f_{2}(\phi),
$$

where $f_{1}(\phi)=2 \sin ^{2}(\phi / 2), f_{2}(\phi)=2 \cos ^{2}(\phi / 2)$ with $\phi$ denotes the angle between the strut 'b' and 'c' (For a perfect honeycomb $\phi=120^{\circ}$ ). In the above equation $\xi_{1}$ as well as $\xi_{2}$ are two constants controlling the overall stiffness of the spring arising from axial and bending stiffness of the bars 'b' and 'c'. By substituting $\sin \theta=w /(l-u)$ in Equation (A.5) the lateral inertia can be rewritten as

$$
m \ddot{w}=\left(\frac{E A u}{l(l-u)}-k\right) w+k w_{0}
$$

Combining Equation (A.3) in the above equation of motion in phase I can be written as

$$
m \ddot{w}=\underbrace{\left[\frac{E A}{l^{2}}\left(\frac{l}{\sqrt{l^{2}-w^{2}}}\left(v t-l \cos \theta_{0}\right)+l\right)-k\right]}_{K_{s}} w+k w_{0} .
$$


In the above equation $K_{s}$ can be thought as an equivalent stiffness of the system which is a function of all geometric and material properties as well as the transverse displacement. The nature of transverse displacement can be predicted by investigating the roots of characteristic equation of above differential equation. A positive value of $K_{s}$ leads to distinct positive roots which give an unstable solution. Therefore, $w$ will increase rapidly with time. On the other hand, a negative $K_{s}$ offers distinct imaginary roots which denotes that $w$ will vary according to a sine or cosine function. Furthermore, the amplitude and frequency of the oscillation solely rely on the magnitude of $K_{s}$ which depends on all material, geometric and loading parameters.

There are two phases of the solutions that can be obtained from the above equation. The phase I is associated with inertial enhancement which is characterized by $\dot{u} \geq 0$ (deformation occurring due to axial compression). This phase is also accompanied by transverse displacement i.e., lateral inertia comes into play. After the inertial enhancement the further vertical shortening can be accommodated only by rotation of the strut which is denoted as phase II. The above equation clearly states that the lateral inertia is dependent on the density, modulus of the strut material as well as the resistance offered by the horizontal spring and the initial misalignment of the strut. Vural and Ravichandran (2003) also found the expression of lateral inertia in terms of the strut material and geometric parameters. The present study is significantly different from their model by introducing the resistance offered by the horizontal strut during the collapse evolution. Their model considered the plastic deformation of the strut which is neglected here as we are interested for deformation of elastomeric foam. The present study not only addresses the inertial enhancement but also explains the post inertial enhancement phenomenon i.e., phase II which has never been addressed in the previous literature.

The above equation is a second order nonlinear ordinary differential equation and can 
be solved numerically to evaluate $w$ and $\dot{w}$ with the following initial conditions,

$$
w=w_{0}, \dot{w}=0 \quad \text { at } \quad t=0 .
$$

The vertical force $F_{v}$ experienced by the strut at point $P$ (Fig. A.2) can be evaluated as

$$
F_{v}=F \cos \theta=\frac{E A u}{l} \cos \theta
$$

The inertial enhancement is restricted when axial shortening reaches to its maximum i.e.,

$$
\dot{u}=0
$$

and the associated time is called critical time and is denoted by $t_{c}$. After the inertial enhancement, the strut is subjected to partial unloading due to microbuckling. A competition between microinertia and microbuckling may lead to sudden drop of the force (or plateau stress) which will be discussed in detail. A complete collapse condition of the strut is achieved when the strut becomes completely horizontal $(w=l)$. At this stage the vertical force experienced by the strut $F_{v}$ vanishes (Equation A.10) as $\theta \approx 90^{\circ}$. Note that the present model explicitly accounts all the characteristic features of a cellular specimen. The half length $l$ signifies the cell size while irregularity of cell size can be quantified with the misalignment of the strut i.e., $\theta_{0}$, where $\theta_{0}=0$ refers a perfect honeycomb. Notice that the density of the strut is embedded in mass of the strut as $m=\rho_{s} A l$. The stiffness of the spring $k$ is also a function of geometric and material properties of strut. With an increase in cross-sectional area or decrease in strut length will offer higher stiffness resulting in lesser deformation of the spring. Thus, the model will enable us to explain the macroscopic dynamic response of cellular specimen arising from different micromechanisms involved at cell level for different cell size, Young's modulus, density and cell size irregularity for a wide range of loading. 


\section{A.3 Result and discussions}

The strut model described in last section has been subjected to an impact velocity at the top end and its dynamic response has been studied in detail. The strut is subjected to a dynamic loading using applied velocity. For the simulation, the material for the strut is chosen as polyethylene, a soft polymer with Young's modulus $E=0.15$ $G P a$ and density $\rho_{s}=910 \mathrm{~kg} / \mathrm{m}^{3}$, unless otherwise noted. The strut is square in crosssection with the thickness $h=2.5 \mu \mathrm{m}$ and half length $l=10 \mu \mathrm{m}$. Note that, length of the strut can be related to the cell size of cellular specimen. Initial misalignment is given by $\theta_{0} \approx 3^{\circ}$ that essentially denotes a moderate randomness in cell size of the cellular material. Mass of the strut $m$ for this configuration is $5.7 \times 10^{-14} \mathrm{Kg}$. The strut is subjected to an impact velocity $v=1.0 \mathrm{~m} / \mathrm{s}$ resulting a strain rate of $10^{5} / \mathrm{s}$. Fig. A.3 shows the simultaneous evolution of normalized axial compression $(u / l)$, normalized transverse displacement $(w / l)$ and the vertical force $\left(F_{v}\right)$ experienced by the strut as shown in Fig. A.2. As explained in previous section, the deformation of strut can be divided in to two phases, where phase I is corresponding to microinertial enhancement and phase II signifies the microbuckling (rotation) of the strut. The transition between the two phases is marked by a dashed line in Fig. A.3. The phase I can further be divided into two subregimes which are also indicated in Fig. A.3. In the regime I (R-I), there is no lateral movement of the strut due to lateral inertia imposed on the system by mass $m$. In this regime, the bar is compressed and the stress level of the strut rises rapidly. Once the lateral inertia is overcome, the strut starts to rotate and a significant amount of transverse displacement $w$ can be observed. This regime is denoted as regime II (R-II) of phase I in Fig. A.3. The axial compression of the strut $u$ further increases, albeit at a decreasing rate, and reaches to a maximum limit which is already defined as the end of phase I or microinertial strength enhancement phase. After this phase, the strut undergoes partial unloading which is denoted as phase II. It can be clearly seen that in this phase, further rotation of the strut takes place and the 


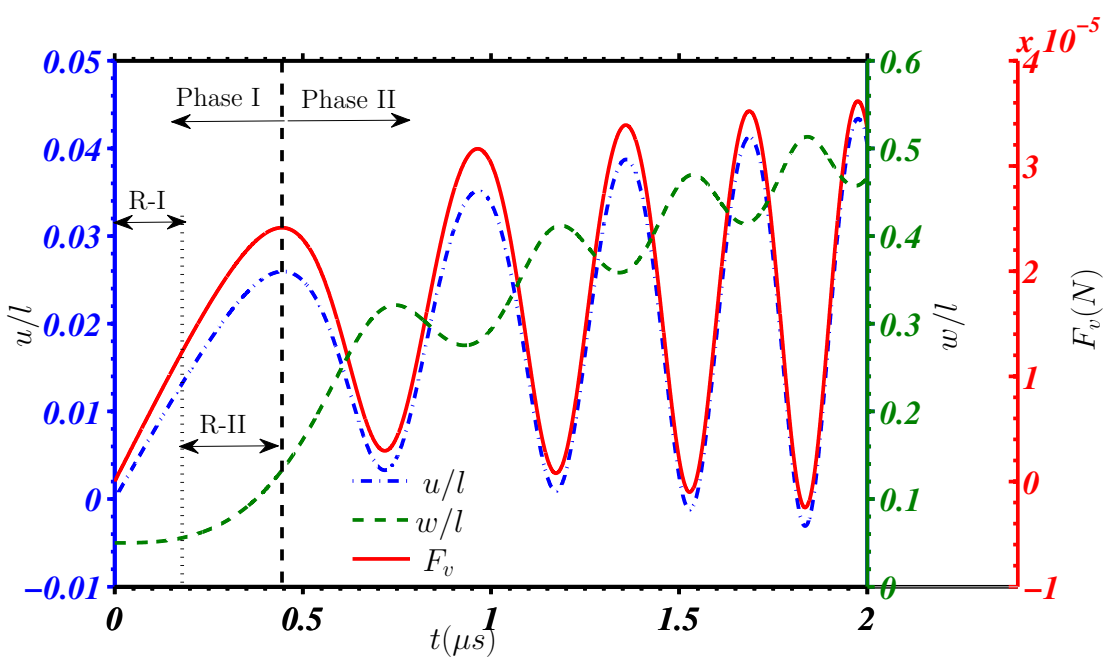

Figure A.3: History of normalized axial compression $(u / l)$, normalized transverse displacement $(w / l)$ and vertical force $\left(F_{v}\right)$ experienced by the strut. Phase I corresponds to the microinertial enhancement, whereas Phase II indicates the microbuckling of the strut.

axial force reduces rapidly. A competition between two opposing effects of microinertial enhancement and partial unloading is responsible for overall softening response of the strut. In a macroscopic sense, phase II corresponds to the post-peak response region in a typical stress strain curve of cellular specimen where microbuckling (and microbending) of cell wall is the only contributing micromechanism. Significant load drop at the cell level is responsible for the macroscale stress softening after the inertial strength enhancement as found in several experimental observations (Zhao et al., 2005; Lee et al., 2006). Note that in the later stage of microbuckling phase (phase II), the strut is subjected to a rotation along with an oscillatory compression. This oscillatory response at the cell level, when averaged over entire domain, gives rise to the macroscopic plateau behavior of the overall stress-strain response. Moreover, the macroscopic oscillation in the plateau region is a superposed behavior of all responses with different frequencies, amplitude and phase differences originating from individual struts. Presence of damping in the system reduces the oscillatory behavior drastically as will be explained in Section A.3.6. Experimentally observed oscillatory macroscopic plateau stress (Zhao et al., 2005; Lee et al., 2006; Thomas et al., 2004) 
during impact loading of cellular specimen can be considered as a result of multiple occurrence of such micromechanisms. As mentioned earlier, $K_{s}$ in Equation (A.8) which is a function $l$ as well $\theta_{0}$, controls oscillations with different frequencies and amplitudes in force-displacement response. Due to presence of heterogeneity in the cellular microstructure, each strut offers oscillatory response with different frequencies and amplitudes. This disparity in the cellular microstructure also alters the transition period between microinertial enhancement (phase I) to microbuckling (phase II); hence, phase difference prevails among the responses. In the current simulation, transverse displacement relates to the collapse of the cell, with $w \approx l$ referring to the complete collapse.

In order to understand the influence of various material, loading and geometric parameters on macroscopic deformation behavior of the cellular specimen, a wide range of design variables (microstructural, material properties and loading parameters) are selected. Next, a parametric study of these variables has been carried out to understand the contribution of various micromechanisms (microinertial enhancement, microbuckling) at the cell level. In the present study, we are specifically interested in studying the axial shortening $(u)$, transverse displacement $(w)$ and vertical force $\left(F_{v}\right)$ experienced by the strut at the cell level for a variety of impact velocities, strut lengths, material properties (Young's modulus and density) and initial misalignment.

\section{A.3.1 Effect of impact velocity}

To explain the dependency of macroscopic stress strain behavior on strain rates, the strut is subjected to a wide range of impact velocities. Remember that the impact velocity $v$ considered here is a local velocity of point $\mathrm{P}$ as shown in Fig. A.2, whereas the macroscopic applied velocity $\left(V_{m}\right.$ in Fig. A.1(a)) at the boundary may be much different from it due to dissipative nature of cellular materials. A large number of cells 
at different layers can deform at a given point of time giving rise to local gradients in the velocity field. A wide range of local impact velocity $v$ is considered here which will exploit a full spectrum of quasi-static to a dynamically deforming regime of the strut. We consider an impact velocity $v=0.01 \mathrm{~m} / \mathrm{s}$ to $1.0 \mathrm{~m} / \mathrm{s}$ keeping other material and geometrical parameters fixed as mentioned previously. Similar to the previous figure, the history of normalized axial shortening $(u / l)$, normalized transverse displacement $(w / l)$ as well as force-displacement $\left(F_{v^{-}} \Delta\right)$ responses are evaluated for different impact velocities in Fig. A.4. Different phases of deformation as mentioned in Fig. A.3 are indicated in following way in all the plots. The solid circle represents the end of microinertial enhancement phase i.e., phase I. The square mark at all the figure shows the transition between subregimes of phase I as discussed earlier.

Normalized axial shortening $(u / l)$, normalized transverse displacement $(w / l)$ and force-displacement $\left(F_{v}-\Delta\right)$ responses in Fig. A.4 simultaneously offer several interesting features of cellular deformation for a wide regime of loading scenario. At high strain rate, the strut results in a highly oscillatory axial compression with higher rate of compression. Such a rapid compression of strut results in higher peak load (Fig. A.4(c) due to microinertial enhancement. An increase of peak load contributes to significant microinertial strength enhancement as found in several experimental and numerical observation (Vural and Ravichandran, 2003). Furthermore, experimentally and numerically observed oscillations (Ma et al., 2009; Pal et al., 2010; Hönig and Stronge, 2002a) in plateau stress can be originated from highly oscillatory force-displacement behavior at strut level. It can also be found that higher impact velocity not only contributes to the strength enhancement of the strut but also offers higher stiffness. Several experimental observations (Tagarielli et al., 2008; Lee et al., 2006; Thomas et al., 2004) shows such enhancement of stiffness at high strain rates. Higher lateral inertia of the strut associated with higher impact velocity contributes to this enhancement. Moreover, at high strain rate the strut rotates faster. Hence, a quick collapse at cell level occurs. Thus, a progressive collapse of 


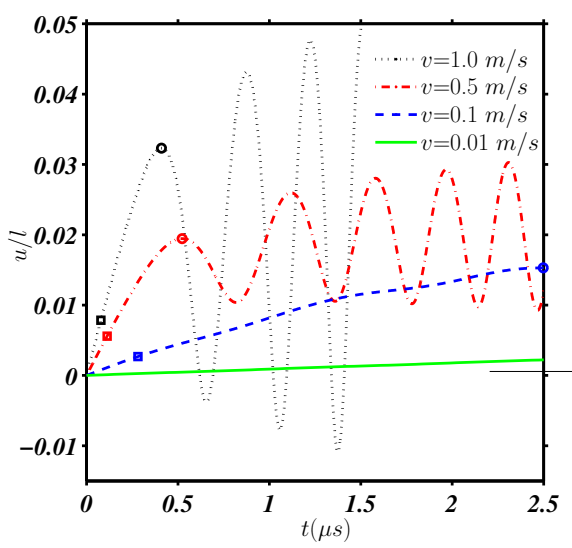

(a)

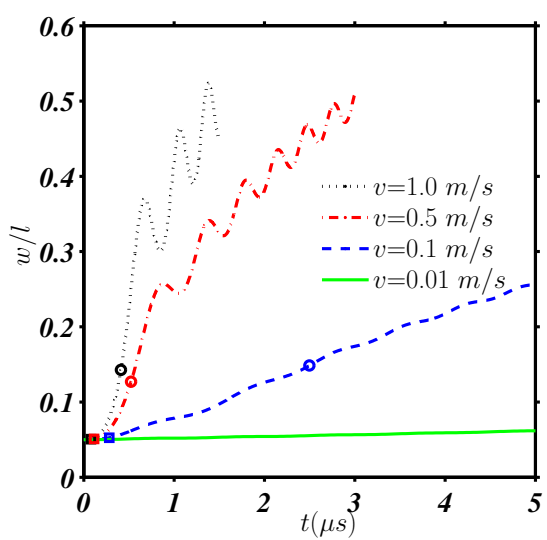

(b)

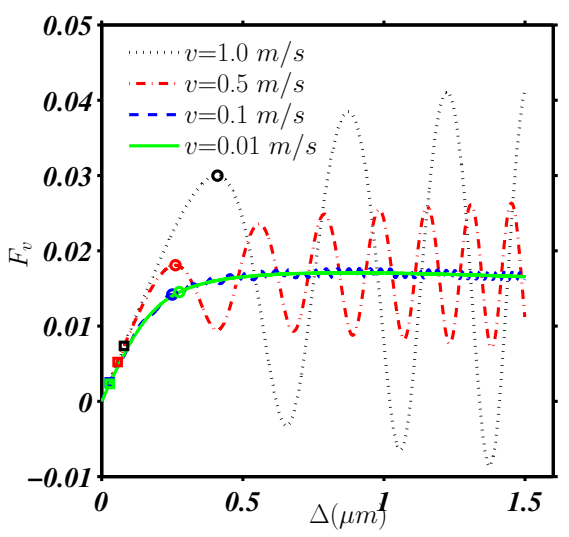

(c)

Figure A.4: (a) History of normalized axial shortening $(u / l)(b)$ normalized transverse displacement $(w / l)$, and (c) vertical force-displacement responses for different impact velocities. The transition from phase I (microinertial enhancement) to phase II (microbuckling) is indicated by solid circle. The subregimes of phase I is also marked by solid squares.

cellular material manifests at high strain rate. In contrast, at lower strain rates, the strut provides a relatively smoother axial compression. Therefore, it gives smooth plateau response. As the microinertial enhancement reduces significantly, it gives lower peak load. Furthermore, strut rotates at a slower rate which enables structure to adjust the applied deformation hence a low force transmission occurs which results in an uniform collapse of the cellular specimen. It can be found that the model is also capable of accurately capture the quasi-static response when the impact veloc- 
ity is reduced considerably $(v=0.01 \mathrm{~m} / \mathrm{s})$. The normalized axial shortening $(u / l)$, normalized transverse displacement $(w / l)$ and force-displacement response $\left(F_{v}-\Delta\right)$ corresponding to the quasi-static case is represented by the solid line. It can be seen that, in quasi-static limit the axial compression as well as transverse displacement are significantly lower. It can be noticed that strut offers a smooth flat response with no inertial enhancement. Note that, the quasi-static collapse force will be used to quantify microinertial strength enhancement of the strut in the subsequent discussions. Finally, it can be concluded that as the impact velocity increases from quasi-static to dynamic regime, a significant rate effect (enhancement of strength and stiffness) emerges at cell level due to lateral inertia offered by individual strut. In addition to the strength enhancement, higher strain rate develops a progressive collapse of the cellular material. In the subsequent analysis, microinertial enhancement and microbuckling will be characterized for different material and microstructural properties for a fully quasi-static to a dynamic regime. Furthermore, the type of macroscopic deformation (progressive or uniform collapse) will be envisaged.

\section{A.3.2 Effect of strut length}

To investigate the effect of cellular microstructural properties on the micromechanisms, the half strut length $l$ is varied. As mentioned earlier, strut length provides a relative measure of the cell size. A wide range of half length $l=5.0$ to $30 \mu \mathrm{m}$ is subjected to impact velocity from $v=0.01$ to $v=1.0 \mathrm{~m} / \mathrm{s}$ to examine the deformation characteristics from a fully quasi-static to dynamic loading regime. As found from the previous analysis, $v=0.01 \mathrm{~m} / \mathrm{s}$ exhibits a fully quasi-static deformation of the strut (no significant effect of lateral inertia can be observed), consequently, we obtain the quasi-static collapse force $F_{q s}$ of the strut for different half strut length $l$ in Fig. A.5(a). To find the exact dependence of the quasi-static collapse force $\left(F_{q s}\right)$ 


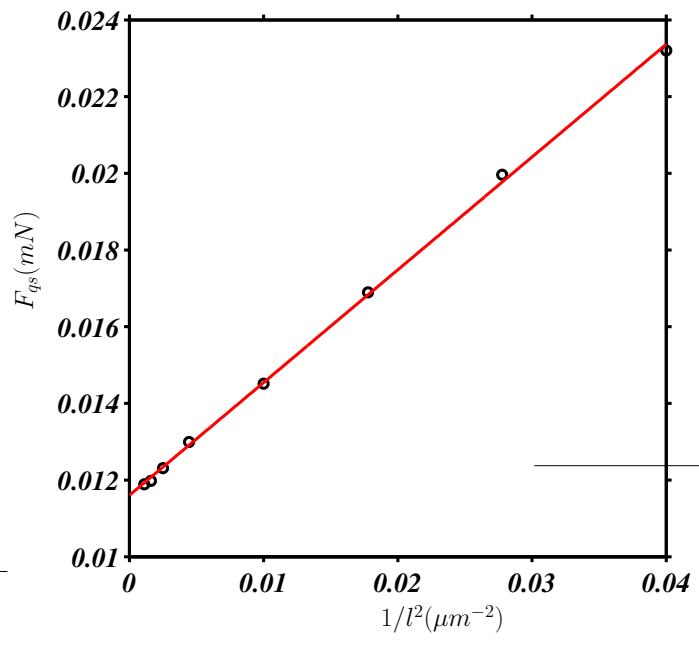

(a)

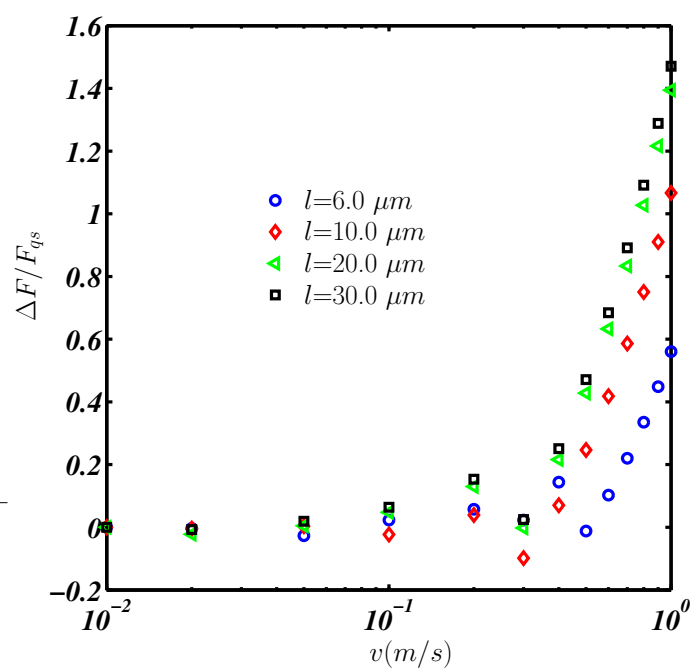

(b)

Figure A.5: (a) Quasi-static collapse force $F_{q s}$ for different half strut length l. (b) Microinertial enhancement of collapse force $\Delta F / F_{q s}$ for different impact velocities with different strut length.

with the strut length, we plot $F_{q s}$ with respect to $l^{-2}$. It can be found that $F_{q s}$ is proportional to $l^{-2}$. Therefore, quasi-static collapse force reduces rapidly with strut length $l$. With reduction of strut length, the stiffness of the strut as well as the spring (equivalent stiffness of the connecting strut) increases which provides higher resistance to the deformation. Consequently, the collapse force of the strut increases with strut length. As the cell size $d_{a v} \propto l$, consequently, $F_{q s} \propto d_{a v}^{-2}$; therefore, it can be inferred that cellular material with higher cell size offers a lower collapse stress. Such an observation is consistent with several numerical and experimental observations (Pal et al., 2010; Brezny and Green, 1990). Furthermore, for a honeycomb type cellular specimen, the density of cellular material $\rho$ can be assumed to be inversely proportional to $d_{a v}$. So, it can be conjectured that peak stress of cellular material depends directly with the square of its density i.e., $F_{q s} \propto \rho^{2}$. Such an observation was also confirmed by Chen et al. (2002d) for polymeric cellular materials.

In order to analyze the contribution of lateral inertia on the force displacement response, the impact velocity is varied from $v=0.01$ to $v=1.0 \mathrm{~m} / \mathrm{s}$. Fig. A.5(b) illustrates 
the microinertial enhancement of different struts for the previous range of impact velocity. In the present study, enhancement of the strength is quantified as $\Delta F / F_{q s}$ where, $\Delta F=F_{\text {peak }}-F_{q s}$, measures the increase of peak force $F_{\text {peak }}$ over its quasi-static collapse force $F_{q s}$. It can be found that for all strut lengths considered in the present study, no rate sensitivity can be observed below $v=0.1 \mathrm{~m} / \mathrm{s}$. Lateral inertia of the strut starts to play a significant role at a velocity $v>0.1 \mathrm{~m} / \mathrm{s}$. It can be found that for a larger strut length, the microinertial enhancement $\left(\Delta F / F_{q s}\right)$ increases rapidly with impact velocity ( $150 \%$ increase with $v=1.0 \mathrm{~m} / \mathrm{s}$ ). As the strut length increase the mass of strut increases which essentially offers a higher lateral inertia and subsequently an enhancement of the strength takes place. Moreover, as microbuckling increases with larger strut, it promotes collapse of strut at cell level. Therefore, a progressive collapse may arise at macroscale with larger strut. On the otherhand, a smaller strut length offers a lower enhancement of the strength over its quasi-static value ( $50 \%$ increment with $l=6 \mu \mathrm{m}$ ). Consequently, an uniform collapse of cellular material will take place with smaller cell size. It can also be inferred that a higher softening as well as the oscillation may arise in the macroscopic plateaus stress for higher strut length. Finally, it can be concluded that a larger cell size offers a higher lateral inertia and thus results in higher strength enhancement while offering a lower quasi-static plateau stress.

\section{A.3.3 Effect of cell size irregularity}

Due to fabrication limitations, cellular microstructure contains several degree of variability in its cell sizes. Designing of an efficient energy absorbing component require a thorough understanding of the effect of such variability in cell sizes on dynamic behavior of cellular specimen. As mentioned earlier, the model can explicitly account the cell size irregularity through the initial misalignment angle $\theta_{0}$ as indicated in 
Fig. A.2. A misalignment angle $\theta_{0} \approx 0^{\circ}$ corresponds to a perfect honeycomb structure, whereas a random structure offers a higher misalignment angle. To understand the deformation at cell level for cellular specimen with different cell size irregularities, the misalignment angle $\theta_{0}$ is varied from $0.04^{\circ}$ to $6^{\circ}$ while keeping other material and microstructural parameters constant. An impact velocity from $v=0.01$ to $1.0 \mathrm{~m} / \mathrm{s}$ is considered to investigate the contribution of micromechanisms in cell level for a fully quasi-static to a dynamically deforming regime. Fig. A.6(a) depicts the variation

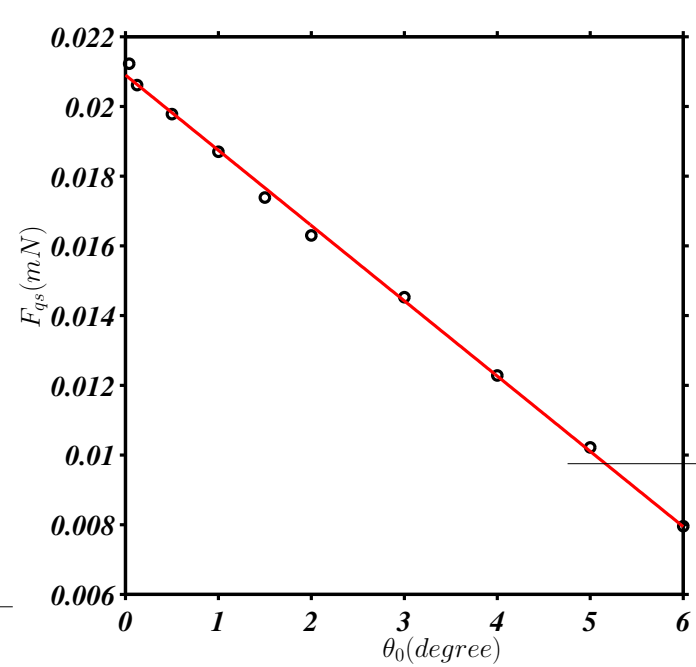

(a)

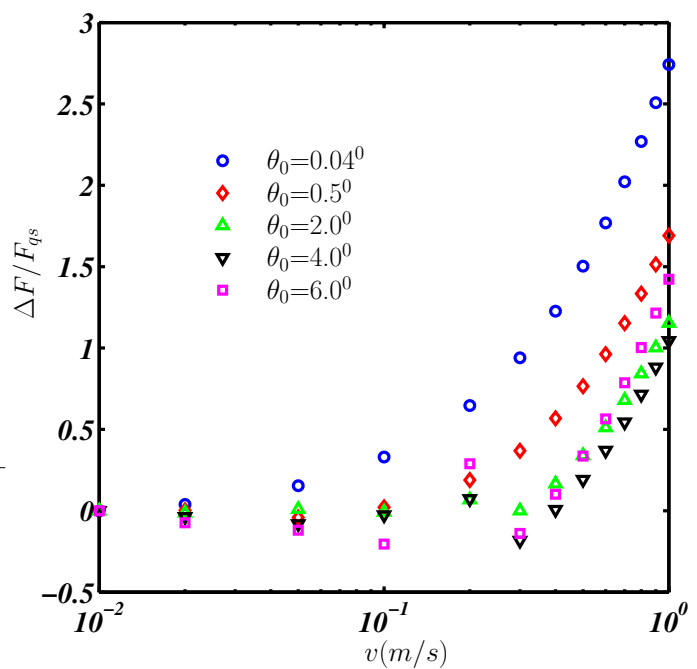

(b)

Figure A.6: (a) Quasi-static collapse force $F_{q s}$ for different misalignment angles $\theta_{0}$. (b) Microinertial enhancement of collapse force $\Delta F / F_{q s}$ for different impact velocities $v$ with different misalignment angles $\theta_{0}$.

of quasi-static collapse force for different initial misalignment angle of the strut. It can be found that collapse force $F_{q s}$ reduces linearly with misalignment angle $\theta_{0}$. In a macroscopic sense, a perfect honeycomb will show higher collapse stress and the stress will reduce with the cell irregularity. In order to analyze the contribution of lateral inertia at cell level associated with randomness in cell size, the microinertial force enhancement is obtained for different initial misalignment angles for a wide range of impact velocity. It can be noted that strut with a very less misalignment angle i.e., $\theta_{0}=0.04^{\circ}$ is much more sensitive to the impact velocity even if at a lower 
velocity $v=0.1 \mathrm{~m} / \mathrm{s}$. A further increase of velocity, the strut offers a significantly higher microinertial strength enhancement. Thus, it can be inferred that a perfect honeycomb structure is mostly sensitive to inertia induced strength enhancement due to higher applied strain rate. As the microbuckling of the strut increases with higher inertial enhancement, deformation localization takes place at cell level due to faster rotation of the strut. Therefore, a perfect honeycomb cellular structure offers early deformation localization and degree of deformation localization is higher compared to random cellular specimens. Hence, the perfect honeycomb structure will exhibit a progressive deformation at macroscopic level. Furthermore, the enhancement of the strength reduces rapidly with the misalignment angle. The strut with a higher misalignment angle $\theta_{0}=6^{\circ}$ corresponding to a highly irregular cell remains almost insensitive to the impact velocity $v=0.3 \mathrm{~m} / \mathrm{s}$. Thus, the extent of deformation localization rapidly reduces with randomness and cellular specimen offers uniform deformation. In a previous study, Su et al. (1995) showed that Type II structure with lower misalignment angle offers rapid enhancement of the strength. Finally, the current model qualitatively predicts that perfect honeycomb cellular material shows higher microinertial strength enhancement along with an oscillatory plateau response and provides progressive deformation localization.

\section{A.3.4 Effect of bulk material density}

In the previous investigations, we have elucidated the effect of geometric parameters on the dynamic response of cellular material. Apart from that, in the following analyses, we attempt to examine the effect of base material properties at the cell level deformation characteristic and its impact on the macroscopic deformation behavior. Therefore, we focus our attention on the density and Young's modulus of the base material. Initially, we vary the density of the bulk material of the strut from $\rho_{s}=600$ 


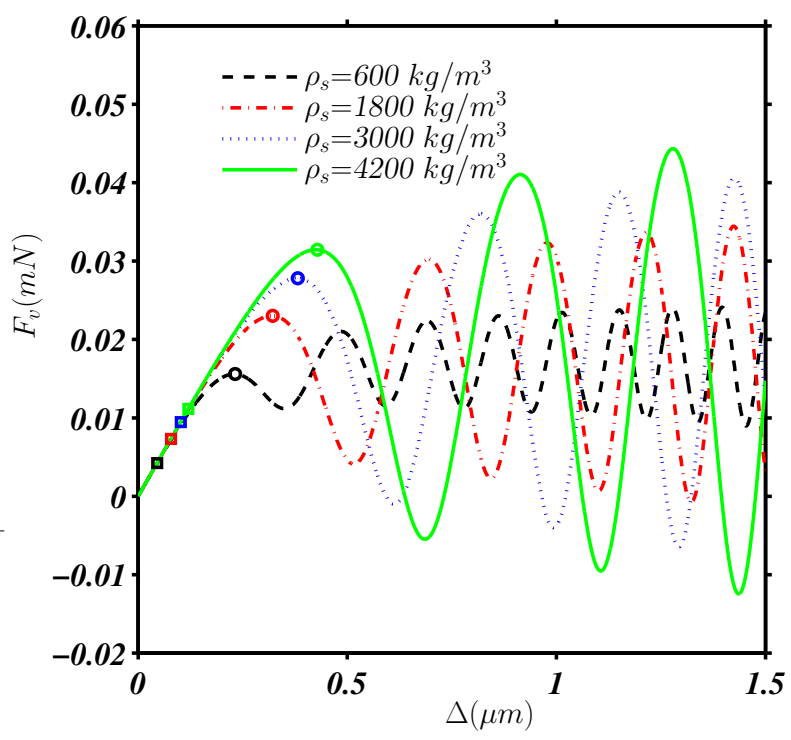

Figure A.7: Vertical force-displacement response for different bulk material densities.

to $6000 \mathrm{~kg} / \mathrm{m}^{3}$ keeping other material and microstructural parameters constant as mentioned earlier. Fig. A.7 shows the force-extension response of the strut with an impact velocity $v=0.5 \mathrm{~m} / \mathrm{s}$. It can found that as the density of the strut increases the inertial enhancement increases. As the density of the strut increases, it offers more inertial resistance to the movement in lateral direction results in such enhancement. A higher microinertial enhancement results in higher microbuckling of the strut at current level and thus it promotes the deformation localization. Moreover, it restricts the energy to flow to next level of strut and consequently, a progressive deformation can be conjectured at macroscopic level of cellular specimen. In order to capture the effect of bulk material density on the microinertial enhancement from a fully quasi-static to a dynamically deforming regime, a wide range of impact velocity is considered here. Fig. A.8(a) illustrates the variation of collapse force $\left(F_{v}\right)$ for different base material densities during quasi-static compression. As expected the bulk material density of strut does not play any role in the quasi-static regime, the peak force remains almost unchanged with density variation. Fig. A.8(b) characterizes the contribution of lateral inertia to the force enhancement associated with density variation 
for a fully quasi-static to a dynamically deforming regime. It can be seen that, upto velocity $v=0.1 \mathrm{~m} / \mathrm{s}$ no struts exhibit microinertial enhancement. Therefore, the cellular specimen will offer a homogeneous macroscopic deformation. At higher velocity $(v>0.1 \mathrm{~m} / \mathrm{s})$ the inertial enhancement becomes significant for higher density specimen. The strut with $\rho_{s}=5400 \mathrm{~kg} / \mathrm{m}^{3}$ with $v=1.0 \mathrm{~m} / \mathrm{s}$ results in a $300 \%$ increase of its collapse force from its quasi-static value while the enhancement is around $50 \%$ for a strut with lower material density $\rho_{s}=600 \mathrm{~kg} / \mathrm{m}^{3}$. As explained in Fig. A.3, a large amount of softening after the inertial enhancement can also be expected with higher density. ? experimentally observed a significant strength enhancement followed by a large amount of softening (load drop) in stress-strain response for a high density polyurethane foam. Similarly, Vural and Ravichandran (2003) experimentally found that the amount of softening in stress-stress response of balsa wood depends on its density. A higher density balsa wood offers a higher amount of softening after the enhancement of peak stress, whereas it is almost negligible for a lower density. As explained earlier higher microinertial enhancement is also followed by several oscillation in the force-displacement response. Consequently, a higher density cellular specimen also results in an oscillatory macroscopic plateau stress with higher amplitude. Thomas et al. (2004) experimentally found that as the density of the cellular core of a sandwich specimen increases during impact loading, the load drop after the inertial enhancement and oscillation becomes more severe and pronounced. Along with a significant amount of softening, an oscillatory stress-stress response for higher bulk material density cellular specimen is also evident in the numerical simulation performed by Pal et al. (2010). In conclusion, the model qualitatively predicts that higher bulk material density cellular specimen provides a significant microinertial strength enhancement as well as it promotes the progressive collapse. 


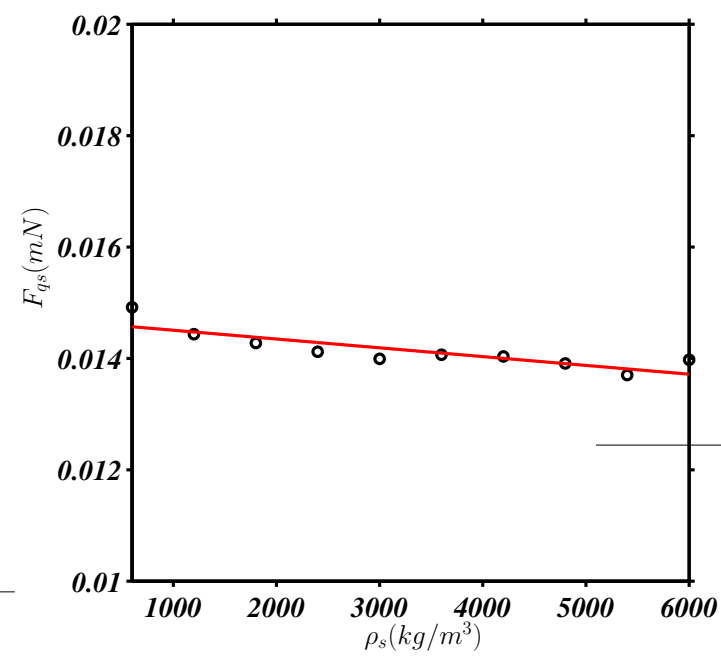

(a)

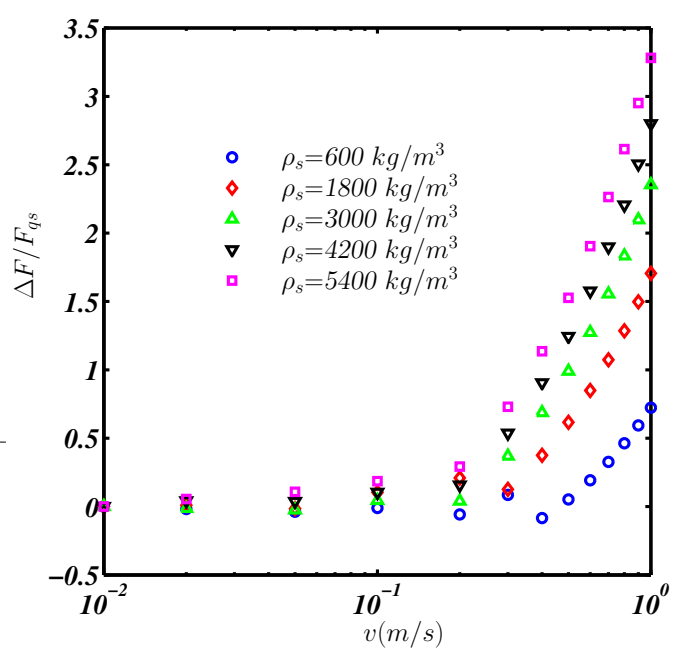

(b)

Figure A.8: (a) Quasi-static collapse force $F_{q s}$ for different bulk material density $\rho_{s}$. (b) Microinertial enhancement of collapse force $\Delta F / F_{q s}$ for different impact velocities $v$ with different bulk material density.

\section{A.3.5 Effect of Young's modulus}

Similar to the previous analysis, we also investigate the effect Young's modulus in microscopic deformation mechanisms of cellular specimen. In order to do that Young's modulus of the strut is varied from $E=0.05$ to $1.5 \mathrm{GPa}$ keeping other parameters as constant. Fig. A.9(a) presents the variation of collapse force of the strut for different Young's modulus in a fully quasi-static regime $(v=0.01 \mathrm{~m} / \mathrm{s})$. It can be seen that as the Young's modulus increases the collapse force increases. With a lower modulus the axial deformation of the strut reaches to its maximum quickly which leads to a lower quasi-static collapse force. Numerical simulation performed by Pal et al. (2010) shows that macroscopic collapse stress varies linearly with the Young's modulus of the bulk materials. To characterize the contribution of Young's modulus of the strut on the lateral inertia, we vary the impact velocity $v=0.01$ to $1.0 \mathrm{~m} / \mathrm{s}$. Fig. A.9(b) indicates that no enhancement can be noticed upto to a velocity $v=0.3 \mathrm{~m} / \mathrm{s}$. Therefore, no cell level collapse takes place and the energy will be transmitted to the next level of cell. 


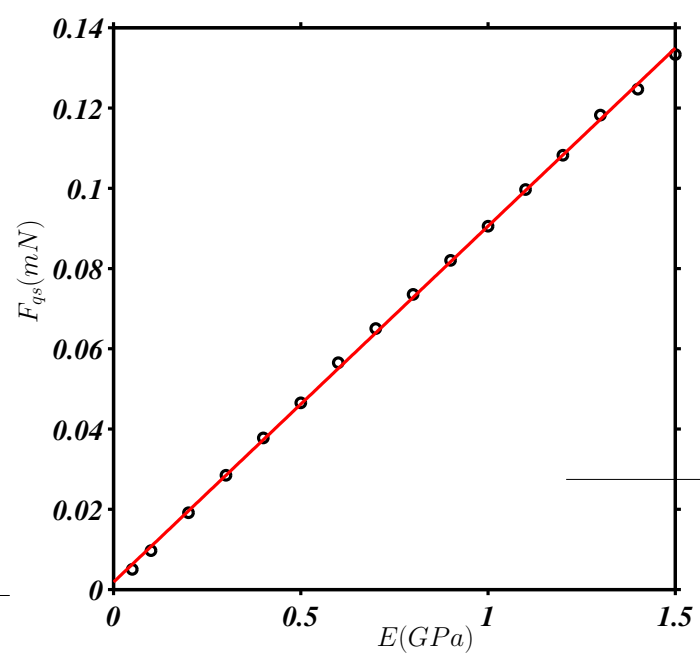

(a)

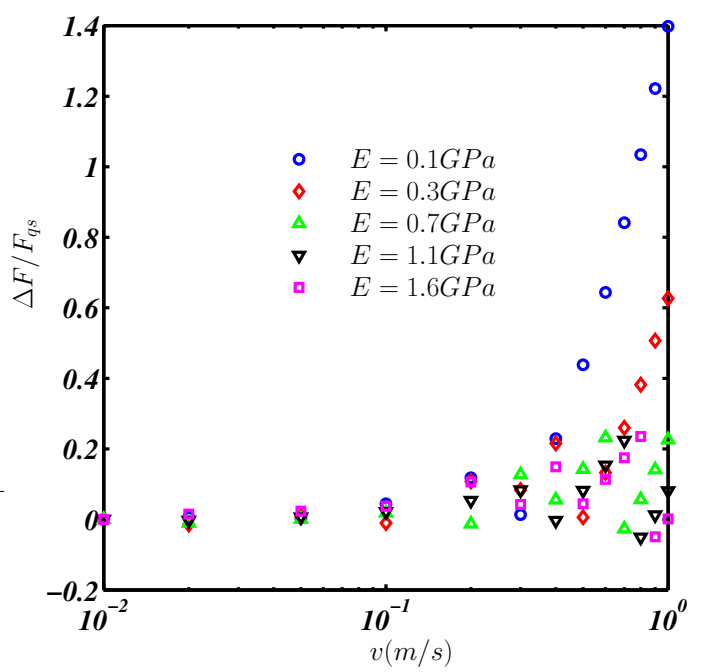

(b)

Figure A.9: (a) Quasi-static collapse force $F_{q s}$ for different Young's modulus of the strut $E$. (b) Microinertial enhancement of collapse force $\Delta F / F_{q s}$ for different impact velocities with different Young's modulus.

Thus, the cellular specimen will offer an uniform macroscopic deformation. Although, at a higher velocity $v>0.3 \mathrm{~m} / \mathrm{s}$, that strut with lower modulus ( $E=0.1 G P a)$ shows an inertial enhancement which is lesser compared to the material density. Moreover, with an increase of Young's modulus, the microinertial enhancement rapidly reduces. With $E>0.7 \mathrm{GPa}$ the strut shows an insignificant amount of microinertial enhancement $(<20 \%)$ as indicated by scattered data. Therefore, the present study signifies that lateral inertia plays a role only for a softer strut and promotes deformation localization, but in lesser extent. Consequently, it can be inferred that for a softer cellular solids, macroscopic response is more sensitive to the strain rates. It is also evident from the present study that a softer cellular material will offer a higher softening and an oscillatory stress-strain response. 


\section{A.3.6 Effect of viscosity}

Viscous dissipative nature of the cellular base material as well as viscous effect caused by the fluid environment in case of fluid filled porous material may play a significant role in the microinertial strength enhancement through offering a smooth plateau response. In order to investigate the effect of such external (due to fluid environment) and internal (inherent dissipation) viscous effects of cellular material during dynamic loading, we introduce the viscous dissipation term $F_{d}=\eta \dot{w}$ in Equation (A.5), where $\eta$ is the viscosity of the either base material or surrounding fluid. In this analysis, we vary the viscosity parameter $\eta=0.0$ to $0.3 \mathrm{e}-6 \mathrm{~Pa}-s$ while keeping the other material and microstructural parameters constant. We consider an impact velocity $v=1.0 \mathrm{~m} / \mathrm{s}$. Fig. A.10(a) shows the force displacement response for different viscosity parameter. In the figure the solid curve is corresponding no internal dissipation.

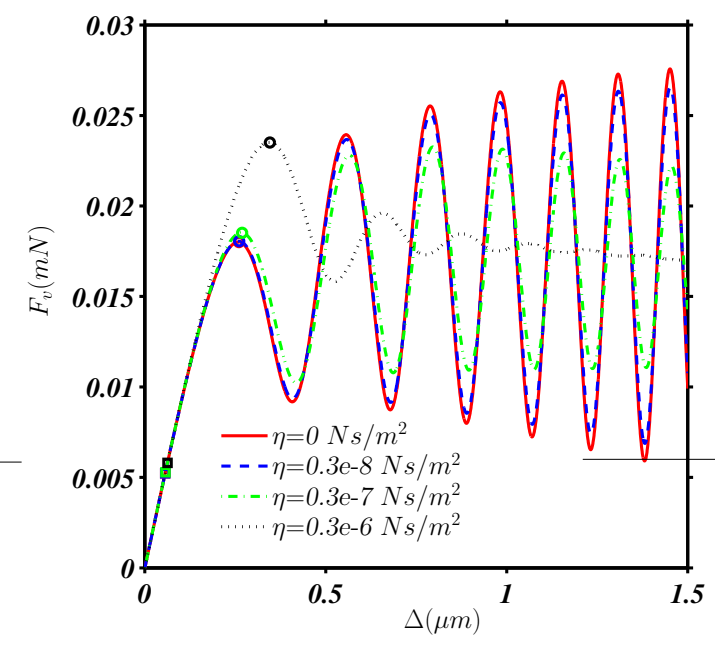

(a)

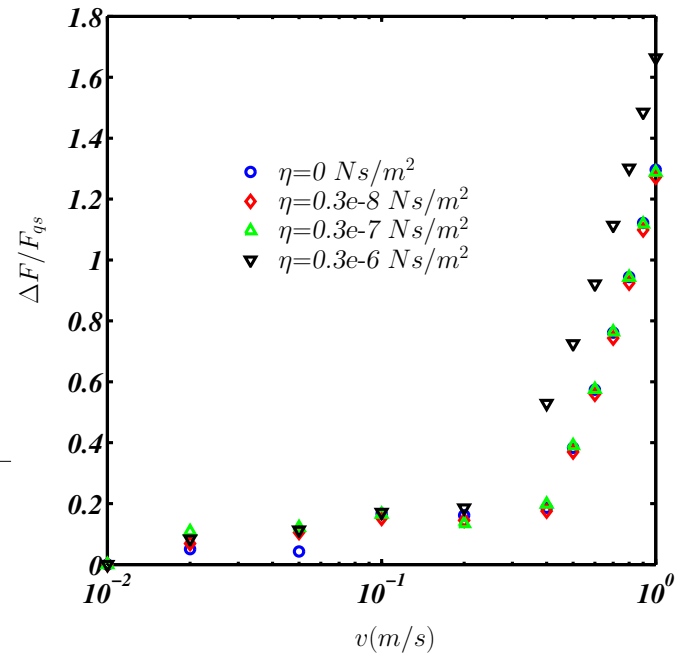

(b)

Figure A.10: (a) Effect of material viscosity $\eta$ on force-displacement response at higher impact velocity $v=1.0 \mathrm{~m} / \mathrm{s}$. (b) Microinertial enhancement of collapse force $\Delta F / F_{q s}$ for different viscosity $\eta$.

It can be seen that when the viscosity is limited to $0.3 \mathrm{e}-7 \mathrm{~Pa}-\mathrm{s}$ oscillations in the microbuckling phase reduce gradually while keeping the enhancement force almost 
unchanged. When a higher of viscosity is considered $\eta=0.3 \mathrm{e}-6 \mathrm{~Pa}-s$, the oscillation dampens out quickly. On the other hand, higher viscosity increases the enhancement strength significantly. In order to investigate the effect of viscosity from a fully quasistatic to dynamically deforming regime, we extract the microinertial enhancement in Fig. A.10(b). It can be found that viscosity does not play any significant role upto an impact velocity $v=0.1 \mathrm{~m} / \mathrm{s}$. A further increase of impact velocity results in significant force enhancement. As the impact velocity increases, the viscous resistance becomes significant which leads further microinertial enhancement. Romero et al. (2008) also showed that higher collapse stress can be achieved for higher viscosity of the cellular base material at high strain rates. Similarly, several biological materials such as bone and muscle are often considered as a cellular structure with a fluid environment (McElhaney, 1966) are sensitive to high strain rate. Carter and Hayes (1976) showed human bone with marrow offers higher compressive strength than a

bone without marrow. Sparks and Dupaix (2008) experimentally observed the stress enhancement in dynamic response of human liver under high strain rate impact. Such stress enhancement at high strain rate can be attributed from the viscous resistance offered by the fluid. From this analysis, it can be concluded that the viscosity may offer strength enhancement in the macroscopic response as well as a smooth plateau behavior during dynamic testing of cellular materials.

\section{A.4 Concluding remarks}

In this chapter, a simplified analytical model has been developed to analyze the deformation response of cellular structure at the strut level during dynamic loading conditions. The model is robust enough to capture the different regimes of response due to microinertia and microbuckling. Contribution of microinertia and microbuckling on the overall response of strut is explained. It is found that the model can 
predict dependence of macroscopic deformation response on cellular material design parameters as found in previous literature. Material and geometrical properties are systematically varied to understand the deformation mechanisms at cell level and to predict the overall response at specimen level. The following conclusions can be inferred from the present analysis:

1. Macroscopic dynamic stress-strain curve of cellular specimen can be considered as a superposed behavior of all responses arising from combined effect of microinertia and microbuckling at strut level.

2. A significant amount of dynamic strength enhancement as well as softening of cellular specimen takes place with higher impact velocity. A highly oscillatory plateau response can also be expected with higher impact velocity.

3. Cellular specimen with higher cell size ( larger strut length) offers higher inertial enhancement as well as stress softening. A perfect honeycomb cellular specimen is more sensitive to high strain rate than a randomized one. It is observed that quasi-static collapse stress is inverse proportional to the square of cell size.

4. Higher bulk material density of the cellular specimen offers a higher inertial enhancement as stress softening. Strut with lower Young's modulus are most sensitive to the inertial enhancement.

5. Perfect honeycomb structure shows higher inertial enhancement and highly oscillator response. The inertial enhancement reduces as the cellular structure is randomized.

6. Viscous resistance in cellular specimen offers higher microinertial enhancement as well as a smooth plateau response at high strain rate. Such viscous resistance 
may be responsible for strength enhancement in dynamic response of several biological materials under impact loading scenario. 


\section{Appendix B}

\section{Voronoi cell generation for cellular microstructure}

The cellular microstructure is constructed based on voronoi tessellation. The detail steps of voronoi microstructure generation with controlled randomness are given below:

- Step 1: A regular hexagonal lattice $\left(x_{i}, y_{i}\right)$ is generated on a plane as shown in Figure B.1(a) with a specified size $d_{0}$.

- Step 2: Hexagonal lattice is perturbed from their original position as

$$
\begin{array}{r}
x_{i}^{\prime}=x_{i}+\zeta d_{0} \cos \theta_{i} \phi_{i}, \\
y_{i}^{\prime}=y_{i}+\zeta d_{0} \sin \theta_{i} \phi_{i},
\end{array}
$$

where, $\theta_{i} \in\left[\begin{array}{ll}0 & \pi\end{array}\right]$ and $\phi_{i} \in\left[\begin{array}{ll}-1 & 1\end{array}\right]$ are two random parameters distributed uniformly. The amplitude of perturbation is controlled by the parameter $\zeta$. 


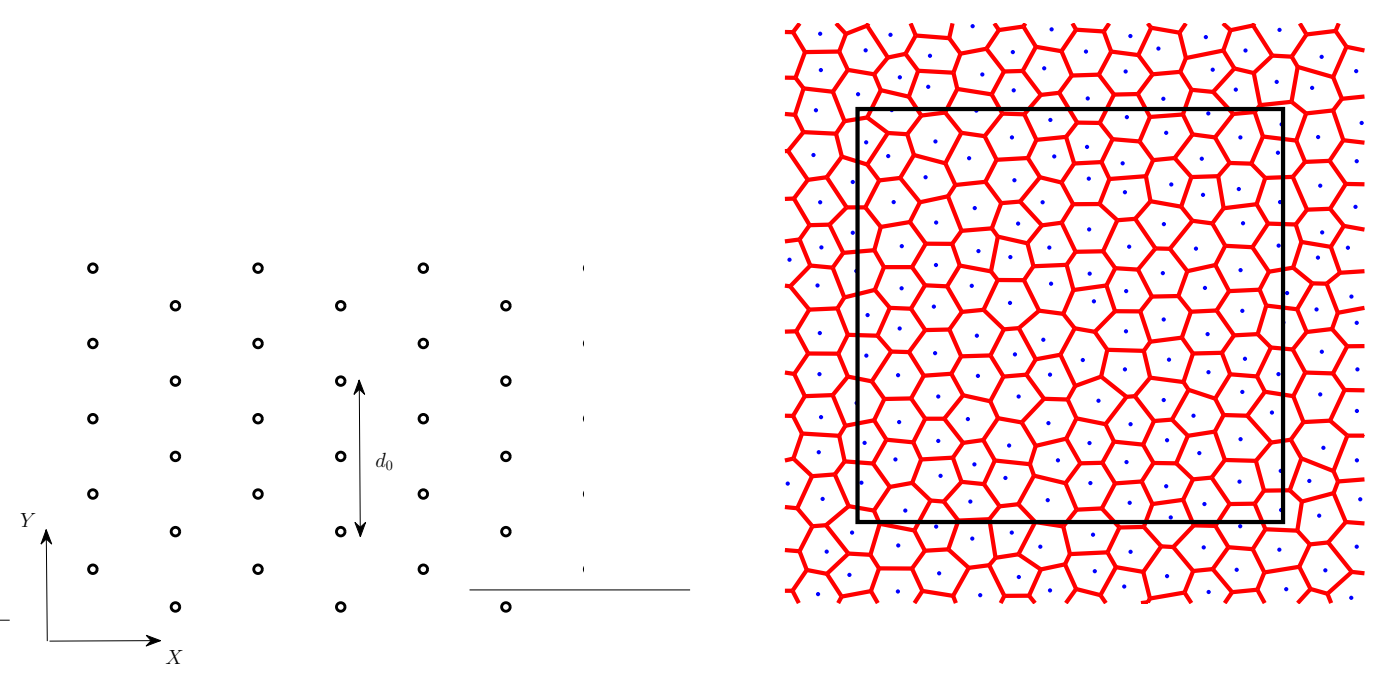

Figure B.1: (a) Hexagonal lattice (unperturbed) on x-y plane. (b) Constructed voronoi diagram on perturbed seeds. Window represents the actual cellular domain taken the diagram.

- Step 3: Using voronoi tessellation algorithm (convex hull), voronoi diagram is constructed on the perturbed seeds as shown Figure B.1(b). A honeycomb cellular structure can be generated by assigning $\zeta=0$. A higher value of $\zeta$ $(=0.25)$ will produce a highly irregular cellular microstructure.

- Step 4: Cellular microstructure with required size as shown in the Figure B.1(b) is obtained from the diagram. The randomness of cellular structure is evaluated through the distribution of individual cell area. 


\section{Appendix C}

\section{Plasticity algorithm}

\section{C.1 Governing equations}

By considering associated flow rule and the von Mises type yield criterion, rateindependent plastic response is characterized as follows:

$$
\begin{gathered}
f(\boldsymbol{\sigma}, \boldsymbol{\kappa}) \leq 0 \quad \text { (yield condition), } \\
\dot{\boldsymbol{\kappa}}=\dot{\lambda} \mathbf{h} \quad \text { (plastic hardening law), } \\
\dot{\boldsymbol{\varepsilon}}^{p}=\dot{\lambda} \frac{\partial f}{\partial \boldsymbol{\sigma}} \quad \text { (Prandtl-Reuss flow rule), }
\end{gathered}
$$

where $\dot{\lambda}$ is the plastic consistency parameter and $\mathbf{h}$ is the vectorial hardening function. Furthermore, the loading/unloading conditions can be expressed in a compact form by requiring that

$$
\dot{\lambda} \geq 0, \quad f(\boldsymbol{\sigma}, \boldsymbol{\kappa}) \leq 0 \text {, and } \quad \dot{\lambda} f(\boldsymbol{\sigma}, \boldsymbol{\kappa})=0 .
$$


The yield criteria can be stated as

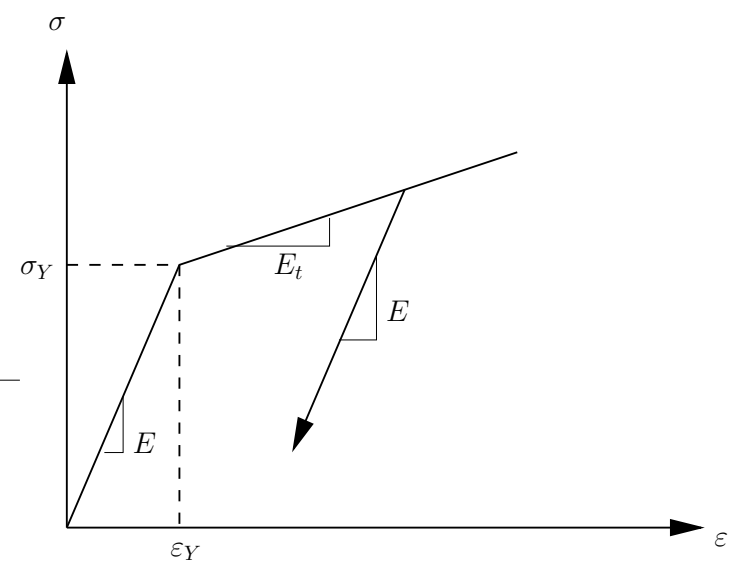

Figure C.1: Bilinear elastic-plastic constitutive law.

$$
f\left(\boldsymbol{\sigma}, \sigma_{y}\right) \equiv F(\boldsymbol{\sigma})-\sigma_{0}\left(\varepsilon_{p s}\right),
$$

where $\sigma_{0}=\sigma_{y}+H \varepsilon_{p s}$, the current yield stress, is a function of material parameter $\sigma_{y}$ and equivalent plastic strain:

$$
\varepsilon_{p s}=\int \dot{\varepsilon}_{p s} d t
$$

The hardening parameter $H$ can be calculated from the uniaxial stress-strain law from Figure C.1 as

$$
H=\frac{\partial \sigma_{0}}{\partial \varepsilon_{p s}}=\frac{E_{t}}{1-E_{t} / E}
$$

The Prandtl-Reuss flow rules associated with the yield function (Equation C.3) is

$$
\dot{\varepsilon}=\dot{\lambda} \frac{\partial f}{\partial \boldsymbol{\sigma}}=\dot{\lambda} \mathbf{a} .
$$

The rate of stress can be related to rate of strain as

$$
\dot{\boldsymbol{\sigma}}=\mathbf{C}\left(\dot{\boldsymbol{\varepsilon}}-\dot{\varepsilon_{p}}\right)=\mathbf{C}(\dot{\boldsymbol{\varepsilon}}-\dot{\lambda} \mathbf{a}) .
$$




\section{C.2 Numerical implementation}

A wide range of algorithm is available to integrated the above equation. The forwardEuler scheme which avoids the iteration is the simplest one. However, it leads spurious loading unloading as the stress does not lie on the yield surface. A back-ward Euler scheme which is an iterative procedure avoids the above difficulties. The detail formulation of back-ward Euler scheme are illustrated as following. The Backward-Euler

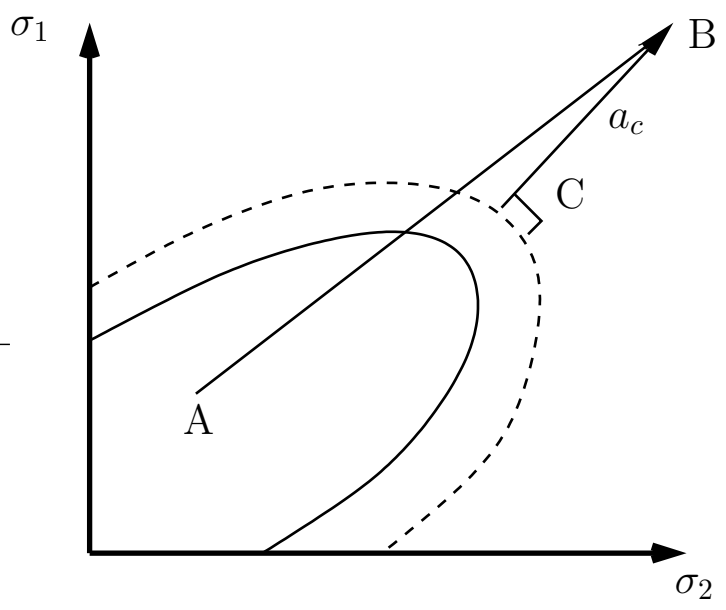

Figure C.2: Backward-Euler scheme. AB corresponds to elastic forward steps, and BC corresponds to the return map on the yield surface.

algorithm shown in the Figure C.2 consists of applying an elastic forward step (AB) followed by a return mapping (BC) on the updated yield surface. The first estimation of $\boldsymbol{\sigma}_{C}$ is calculated with

$$
\boldsymbol{\sigma}_{C}=\boldsymbol{\sigma}_{B}-\Delta \lambda \mathbf{C a}_{B}=\boldsymbol{\sigma}_{A}+\mathbf{C} \Delta \boldsymbol{\varepsilon}-\Delta \lambda \mathbf{C} \mathbf{a}_{B}
$$

where $\mathbf{a}_{B}$ is calculated from Equation (C.6) and $\Delta \lambda$ is derived as following. A first order Taylor expansion of the yield function around point B is given as

$$
f=f_{B}+\frac{\partial f^{T}}{\partial \boldsymbol{\sigma}} \Delta \boldsymbol{\sigma}_{B}+\frac{\partial f}{\partial \sigma_{0}} \frac{\partial \sigma_{0}}{\partial \varepsilon_{p s}} \Delta \varepsilon_{p s}=f_{B}+\mathbf{a}_{B}^{T} \Delta \boldsymbol{\sigma}_{B}-H \Delta \lambda .
$$


Equation (C.6) is then used to find $\Delta \boldsymbol{\sigma}_{B}$ with $\Delta \varepsilon=0$ because the total strain has already been applied in the elastic step (AB). Therefore, $\Delta \boldsymbol{\sigma}_{B}$ can be found as

$$
\Delta \sigma_{B}=\mathbf{C}\left(\Delta \varepsilon-\Delta \lambda \mathbf{a}_{B}\right)=-\Delta \lambda \mathbf{C} \mathbf{a}_{B} .
$$

If the new yield function value $f$, is to be zero, Equation (C.9) alongwith Equation (C.10) gives

$$
\Delta \lambda=\frac{f_{B}}{\mathbf{a}_{B}^{T} \mathbf{C a} \mathbf{a}_{B}+H} .
$$

However, this initial estimate will not satisfy the yield function. Hence, further iteration will be required because the normal at the trial position B will not generally equal to the final normal. In order to perform an iterative procedure to evaluate the final stress, a residual vector $\mathbf{R}$ is defined which represents the difference between the current stress and backward-Euler stress i.e.,

$$
\mathbf{R}=\boldsymbol{\sigma}_{c}-\left(\boldsymbol{\sigma}_{B}-\Delta \lambda \mathbf{C} \mathbf{a}_{C}\right),
$$

and iteration are performed to reduce $\mathbf{R}$ to zero while the final stresses must satisfy the yield criteria, $f=0$. Keeping the elastic trial stress $\boldsymbol{\sigma}_{B}$ as constant, a truncated Taylor series expansion can be applied to the above equation so as to produce a new residual, $\mathbf{R}_{n}$ where

$$
\mathbf{R}_{n}=\mathbf{R}_{0}+\dot{\boldsymbol{\sigma}_{C}}+\dot{\lambda} \mathbf{C} \mathbf{a}_{C}+\Delta \lambda \mathbf{C} \frac{\partial \mathbf{a}_{C}}{\partial \boldsymbol{\sigma}_{C}} \dot{\boldsymbol{\sigma}_{C}}
$$

In the above equation $\dot{\sigma}_{C}$ represents the change in $\sigma_{C}$ and $\dot{\lambda}$ denotes the change in $\Delta \lambda$. Setting $\mathbf{R}_{n}=0$ in Equation (C.13) offers

$$
\boldsymbol{\sigma}_{C}=-\left(\mathbf{I}+\Delta \lambda \mathbf{C} \frac{\partial \mathbf{a}_{C}}{\partial \boldsymbol{\sigma}_{C}}\right)^{-1}\left(\mathbf{R}_{0}+\dot{\lambda} \mathbf{C} \mathbf{a}_{C}\right)=-\mathbf{Q}^{-1}\left(\mathbf{R}_{0}+\dot{\lambda} \mathbf{C} \mathbf{a}_{C}\right)
$$


A truncated Taylor series expansion of the yield surface around point $\mathrm{C}$ gives

$$
f_{c}=f_{c 0}+\frac{\partial f}{\partial \boldsymbol{\sigma}_{c}} \dot{\boldsymbol{\sigma}}_{c}+\frac{\partial f}{\partial \sigma_{0}} \frac{\partial \sigma_{0}}{\partial \varepsilon_{p s}} \dot{\varepsilon}_{p s}=f_{c 0}+\mathbf{a}_{c}^{T} \dot{\boldsymbol{\sigma}}_{c}+H \dot{\lambda}
$$

Furthermore, by introducing $f_{c}=0$ in the above equation and using Equation (C.14) $\dot{\lambda}$ can be found as

$$
\dot{\lambda}=\frac{f_{c 0}-\mathbf{a}^{T} \mathbf{Q}^{-1} \mathbf{R}_{0}}{\mathbf{a}_{C}^{T} \mathbf{Q}^{-1} \mathbf{C} \mathbf{a}_{C}+H} .
$$

The numerical implementation of Backward-Euler scheme for rate independent plasticity can be performed through Algorithm 3 .

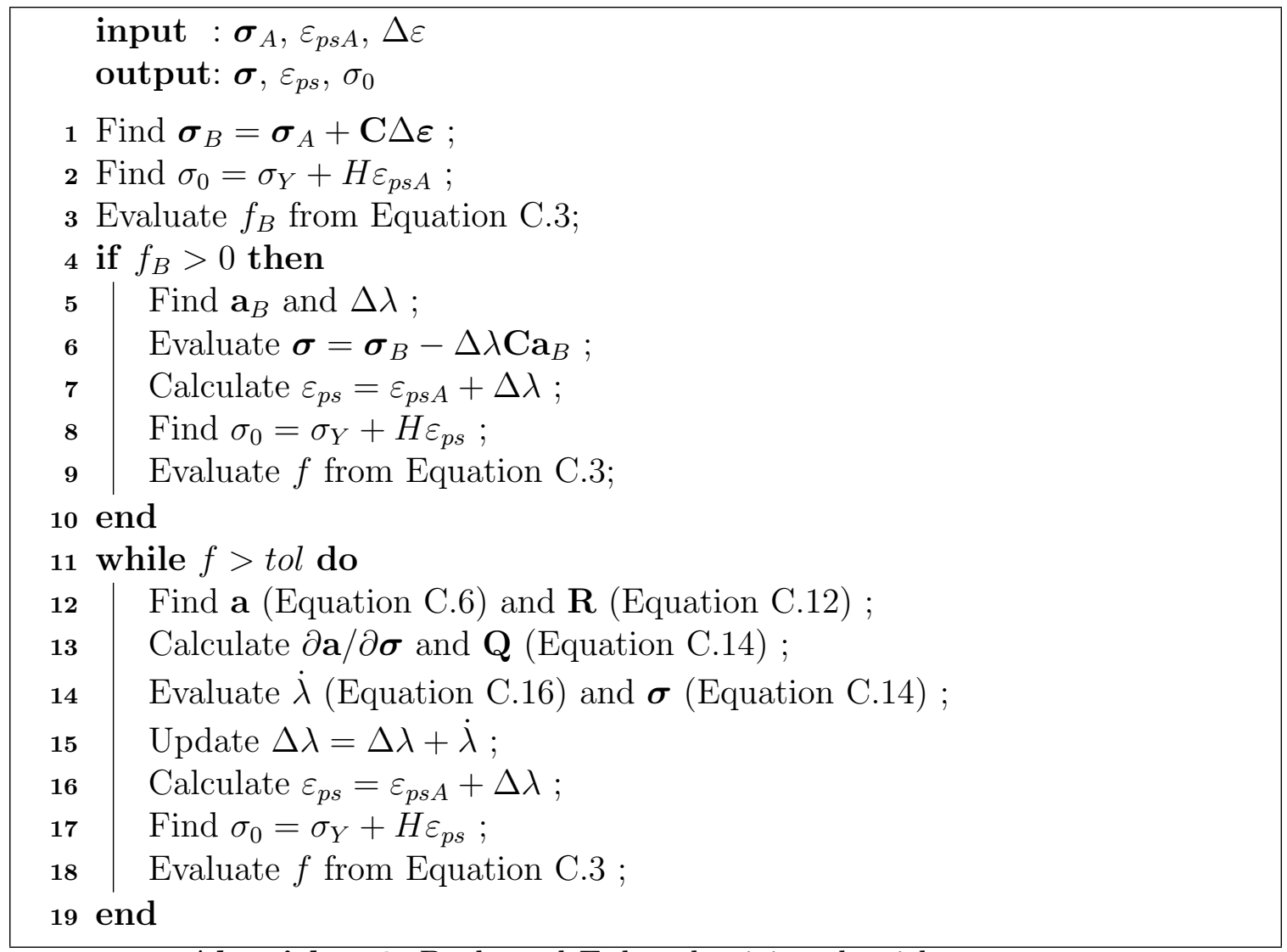

Algorithm 3: Backward-Euler plasticity algorithm. 


\section{Appendix D}

\section{Finite element formulation of large strain hyperelasticity}

\section{D.1 Linearization of equilibrium condition (Lagra- ngian formulation)}

For any admissible displacement field $\delta \mathbf{u} \in H_{0}^{1}\left(\Omega_{0}, t\right)$, the principle of virtual work states that

$$
\mathbf{R}:=\delta W_{i n t}(\mathbf{u}, \delta \mathbf{u})-\delta W_{e x t}(\mathbf{u}, \delta \mathbf{u})=\mathbf{0},
$$

where internal virtual work $\delta W_{i n t}$ and external virtual work $\delta W_{e x t}$ can be found in material description as

$$
\begin{aligned}
\delta W_{\text {int }}(\mathbf{u}, \delta \mathbf{u}) & =\int_{\Omega_{0}} \mathbf{S}: \delta \mathbf{E} \partial \Omega, \\
\delta W_{\text {ext }}(\mathbf{u}, \delta \mathbf{u}) & =\int_{\Omega_{0}} \mathbf{B} \cdot \delta \mathbf{u} \partial \Omega+\int_{\partial \Omega_{0}} \mathbf{t} \cdot \delta \mathbf{u} \partial \Gamma .
\end{aligned}
$$


In the above equation, $\mathbf{S}, \mathbf{B}$ and $\mathbf{t}$ denote second-Piola Kirchoff stress tensor, body force and traction applied at boundary, respectively. The macroscopic Green-Lagrange strain tensor given as $\mathbf{E}=\frac{1}{2}(\mathbf{C}-\mathbf{I})=\left(\mathbf{F}^{T} \mathbf{F}-\mathbf{I}\right) / 2$. An incremental/iterative solution technique is adopted to solve the above nonlinear equation. Linearization of Equation (D.1), i.e., $\mathbf{R}+D_{\Delta \mathbf{u}} \mathbf{R}=\mathbf{0}$, offers

$$
\delta W_{i n t}(\mathbf{u}, \delta \mathbf{u})-\delta W_{e x t}(\mathbf{u}, \delta \mathbf{u})+D_{\Delta \mathbf{u}} \delta W_{i n t}(\mathbf{u}, \delta \mathbf{u})-D_{\Delta \mathbf{u}} \delta W_{\text {ext }}(\mathbf{u}, \boldsymbol{\delta} \mathbf{u})=0
$$

where, $D(\cdot)$ represents the Directional derivative (Gateaux operator). Using the variation of $\mathbf{E}$ can be found as

$$
\delta \mathbf{E}=\frac{1}{2}\left[\mathbf{F}^{T} \nabla_{X}(\delta \mathbf{u})+\nabla_{X}(\delta \mathbf{u})^{T} \mathbf{F}\right] .
$$

in Equation (D.2), the directional derivative of the internal virtual work can be obtained as

$$
D_{\Delta \mathbf{u}} \delta W_{i n t}(\mathbf{u}, \boldsymbol{\delta} \mathbf{u})=\int_{\Omega_{0}} \nabla_{X}(\delta \mathbf{u}): \nabla_{X}(\Delta \mathbf{u}) \mathbf{S} \partial \Omega+\mathbf{F}^{T} \nabla_{X}(\delta \mathbf{u}): \mathbb{C}: \mathbf{F}^{T} \nabla_{X}(\Delta \mathbf{u}) \partial \Omega
$$

For a hyperelastic material, the second Piola-Kirchoff stress tensor and tangent moduli can be extracted from the stress energy potential $\Psi$ as

$$
\mathbf{S}=2 \frac{\partial \Psi}{\partial \mathbf{C}}, \quad \text { and } \quad \mathbb{C}=4 \frac{\partial^{2} \Psi}{\partial \mathbf{C} \partial \mathbf{C}}
$$

As an example, for a compressible Neo-Hookean material the strain energy is given as

$$
\Psi=\frac{\mu}{2}\left(I_{c}-3\right)-\mu \ln J+\frac{\lambda}{2}(\ln J)^{2},
$$

where, $\mu$ and $\lambda$ are the material constant, $I_{c}=\operatorname{tr}(\mathbf{C})$, is the first invariant of $\mathbf{C}$ and $J^{2}=I I_{c}$. Correspondingly, second-Piola Kirchhoff stress tensor $S_{i j}$ and tangent moduli 
$\mathbb{C}_{i j k l}$ can be derived as

$$
\begin{aligned}
S_{i j} & =\lambda \ln (J) C_{i j}^{-1}+\mu\left(\delta_{i j}-C_{i j}^{-1}\right), \\
\mathbb{C}_{i j k l} & =\lambda C_{i j}^{-1} C_{k l}^{-1}+\mu\left(C_{i k}^{-1} C_{j l}^{-1}+C_{i l}^{-1} C_{j k}^{-1}\right) .
\end{aligned}
$$

\section{D.2 Finite element implementation}

Finite element discretization is performed in the initial configuration using isoparametric element to interpolate the reference geometry in terms of nodal position vector $\mathbf{X}_{I}$ defining the initial position of the element nodes as

$$
\mathbf{X}=N_{I}(\boldsymbol{\xi}) \mathbf{X}_{I}
$$

where, $N_{I}(\boldsymbol{\xi})$ is the shape function of $I^{\text {th }}$ node and $\boldsymbol{\xi}=\left(\xi_{1}, \xi_{2}, \xi_{3}\right)$ are the volume (barycentric) coordinates. The position vector $\mathbf{x}$ can be described in terms of current nodal position $\mathbf{x}_{I}$ as $\mathbf{x}=N_{I} \mathbf{x}_{I}$. Correspondingly, the displacement field $\mathbf{u}$ can be written as $\mathbf{u}=\mathbf{N}_{I} \mathbf{u}_{I}$. The derivative of shape function with respect to material co-ordinates are given as

$$
\mathbf{N}_{, \mathbf{X}} \equiv \mathbf{B}^{0}=\mathbf{N}_{, \boldsymbol{\xi}} \mathbf{X}_{, \boldsymbol{\xi}}{ }^{-1}
$$

The jacobian matrix $\mathbf{X}_{, \boldsymbol{\xi}}=\mathbf{X}_{I} \mathbf{N}_{, \boldsymbol{\xi}}$. The deformation gradient tensor $\mathbf{F}$ can be obtained as

$$
\mathbf{F}=\mathbf{x}_{I} \mathbf{B}_{I}^{0^{T}}
$$

Substituting Equation (D.10) and (D.11) in Equation (D.3), it can be rewritten as

$$
\mathbf{K}_{T} \Delta \mathbf{u}=\mathbf{f}^{e x t}-\mathbf{f}^{i n t}
$$


The stiffness matrix can be written as

$$
\mathbf{K}_{T}=\int_{\Omega_{0}} \mathbf{B}^{0^{T}} \mathbf{S B}^{0} \partial \Omega+\int_{\Omega_{0}} \mathbf{F}^{T} \mathbf{B}^{0} \mathbb{C B}_{0}^{T} \mathbf{F} \partial \Omega
$$

The external and internal force vector can be written as

$$
\begin{aligned}
& \mathbf{f}^{i n t}=\int_{\Omega_{0}} \mathbf{B}^{0^{T}} \mathbf{S} \partial \Omega, \\
& \mathbf{f}^{e x t}=\int_{\Omega_{0}} \mathbf{N}^{T} \mathbf{B} \partial \Omega+\int_{\Gamma_{0}} \mathbf{N}^{T} \mathbf{t} \partial \Gamma,
\end{aligned}
$$

\section{D.3 Newton-Raphson solution algorithm and line search method}

The system of linearized equation (Equation D.12) is solved for series of load external load increment or prescribed displacements. For each external load or applied displacement step, the increment nodal displacement is obtained in Newton iterative procedure by solving equation as

$$
\mathbf{K}_{T} \Delta \mathbf{u}^{k+1}=\mathbf{f}_{e x t}^{k}-\mathbf{f}_{i n t}^{k},
$$

where, $k$ represents the iteration index. After obtaining the increments in the nodal displacements, the current displacement is updated as $\mathbf{u}^{k+1}=\mathbf{u}^{k}+\Delta \mathbf{u}^{k+1}$. This iteration procedures continues until it satisfies a convergence criteria. Three types of convergence criteria are used to terminate the iterations: (1) based on the magnitude

of the residual $\mathbf{R}$, (2) based on the magnitude of the displacement increments $\Delta \mathbf{u}^{k+1}$, and (3) based on the energy error criteria. For the present finite element formulation 
displacement increment error criteria is adopted given as

$$
\left\|\Delta \mathbf{u}^{k+1}\right\|_{L_{2}}=\left(\sum_{i=1}^{n}\left(\Delta u_{i}^{k}\right)^{2}\right)^{\frac{1}{2}} \leq \epsilon\left\|\mathbf{u}^{k}\right\|_{L_{2}}
$$

where, $\epsilon=0.001-0.005$.

\section{D.4 Line search algorithm}

During complex deformation, the straight application of Newton-Raphson method becomes insufficient lead to nonconvergence of the solution. Line search method

offers an effective way to improve the convergence. This approach assumes although the direction of $\Delta \mathbf{u}^{k+1}$ found by Newton method is an optimal direction, but the step size $\left\|\Delta \mathbf{u}^{k+1}\right\|$ is not optimal. Therefore, the solution is updated by a parameter $\eta$ as

$$
\mathbf{u}^{k+1}=\mathbf{u}^{k}+\eta \Delta \mathbf{u}^{k+1}
$$

The value of $\eta$ is found such that the total potential energy is minimized with respect to $\eta, d W(\eta) / d \eta=0$. Alternatively, it can be stated that residual $\mathbf{R}$ is orthogonal to the direction of advance $\Delta \mathbf{u}^{k+1}$ which leads

$$
R(\eta)=\Delta \mathbf{u}^{k+1^{T}} \mathbf{R}\left(\mathbf{u}^{k}+\eta \Delta \mathbf{u}^{k+1}\right)=0
$$

This is a nonlinear equation in terms of $\eta$. Therefore, the method of bisection or searches based on interpolation are used to find $\eta$. The most commonly used technique is based on quadratic interpolation (Bonet and Wood, 2008). In this approach, the residual at two point is evaluated, and then the residual measure is interpolated by a quadratic function of $\eta$. The quadratic approximation procedure is repeated until it has been minimized to a desired precision. 


\section{Appendix E}

\section{Genetic algorithm based optimization}

\section{E.1 Optimization formulation}

An optimization formulation can be stated as

$$
\begin{array}{r}
\text { Maximize } \quad f(\mathbf{X}) \\
\text { Subjected to: } \quad g_{i}(\mathbf{X}) \leq b_{i} \\
\mathbf{X}_{i}^{\min }<\mathbf{X}_{i}<\mathbf{X}_{i}^{\max }
\end{array}
$$

where, $f, g_{i}$ and $\mathbf{X}$ represent the objective function or cost function, constraints and system parameters, respectively. The above optimization problem can be solved using direct and gradient based methods. In case of gradient based approach first 
and/or second-order derivatives of the objective function and/or constraints are used to perform the search of solution space. In contrast, only the objective function and constraints are used for direct search method (e.g., Particle Swarm Optimization, Simulated Annealing, Differential Evolution, Genetic Algorithm). As no gradient information is used in case of direct search method, it requires large number of function evaluations. Moreover, gradient based techniques are highly inefficient for nonconvex problem.

\section{E.2 Genetic algorithm}

Genetic algorithm is a robust search and optimization procedures that are based on the underlying principles of natural genetics and natural selection. The terminology used in GA and their mathematical equivalence as well as construction are shown in Table E.1. In brief genetic algorithm starts with randomly chosen parent chromosomes from a pool of population (search space) to generate a new population. Next, genetic operator and genetic processes: selection, recombination and mutation are applied to evolve a better population.

Table E.1: Genetic algorithm terminology

\begin{tabular}{|c|c|c|}
\hline \hline Genetic representation & Mathematical terminology & Representation \\
\hline Generation & Iteration & $N_{\text {gen }}$ \\
Gene & Coded particular variable & $X_{i}:=\{1010101\}_{\text {nbits }}$ \\
Chromosome & Coded vectors of variables & $\Lambda_{i}:=\left\{X_{1} X_{2} . . X_{k} . . X_{N}\right\}$ \\
Population & Set of coded vectors & $\psi:=\left\{\Lambda_{1} \Lambda_{2} \cdots \Lambda_{\text {npop }}\right\}$ \\
$f\left(\Lambda_{i}\right)$ \\
Objective function & Model characteristic & $\bar{f}\left(\Lambda_{i}\right)$ \\
Fitness function & Normalized objective function & $\psi^{\prime}:=\left\{\Lambda_{1}^{\prime} \Lambda_{2}^{\prime} \cdots \Lambda_{\text {npop }}^{\prime}\right\}$ \\
Offspring & New population & $\psi^{\prime}$ \\
\hline
\end{tabular}




\section{E.3 Numerical implementation}

Basic features of GA procedures as given in flowcharts are implemented as following

- Initialization: The solution parameters $\mathbf{X}$ is encoded in a chromosome. First, each variable is represented a string of binary bits as $X_{i}:=\{1010101 \ldots\}_{\text {nbits }}$ with a chosen size of nbits. Next, the chromosome is formed by concatenating all the string to a single string as $\Lambda_{i}:=\left\{X_{1} X_{2} . . X_{k} . . X_{N}\right\}$. Furthermore, a population of candidate solutions $\psi:=\left\{\begin{array}{ll}\Lambda_{1} & \Lambda_{2} \cdots \Lambda_{N p o p}\end{array}\right\}$ are generated for a given number of chromosome Npop.

- Evaluation: The fitness, the value of objective function $\bar{f}\left(\Lambda_{i}\right)$, is obtained for each candidate solution from initial population or the off-springs in subsequent generation.

- Selection: This the first genetic operator that determines the sampling that will produce offspring. This operator provides more copies to the imposing survival of the fittest mechanisms on candidate solutions. A multitude of selection procedure has been proposed to prefer the better solutions such as roulettewheel selection, tournament selection and rank based selection.

- Recombination: Crossover or recombination is the second genetic operator that combines bits and pieces of two or more parental solution to produces new, possible better offspring. The fraction of parents undergoing the crossover is determined by the user defined crossover probability $p_{c}$ and, consequently, with $1-p_{c}$ the chromosome are directly copied to in the offspring. GA includes several recombination schemes: N-point crossover, uniform cross over, arithmetic crossover and blend crossover. 
- Mutation: This is the third evolutionary operator for GA aiming to create new individual from a given chromosome by exchanging one or more genes in it. Mutation performs a random walk in the neighborhood of a candidate solution, thus allows not to trap in local minima. This process is controlled by mutation probability $p_{m}$, an user defined parameter, determines the mutation frequency. A small number is recommended for mutation probability.

- Termination: Number of generations $N_{\text {gen }}$ is considered as the termination criteria of genetic algorithm. The number of generations is so chosen such that there is no change in the parents and offspring.

A flowchart showing the detail steps of GA procedure is given below. 


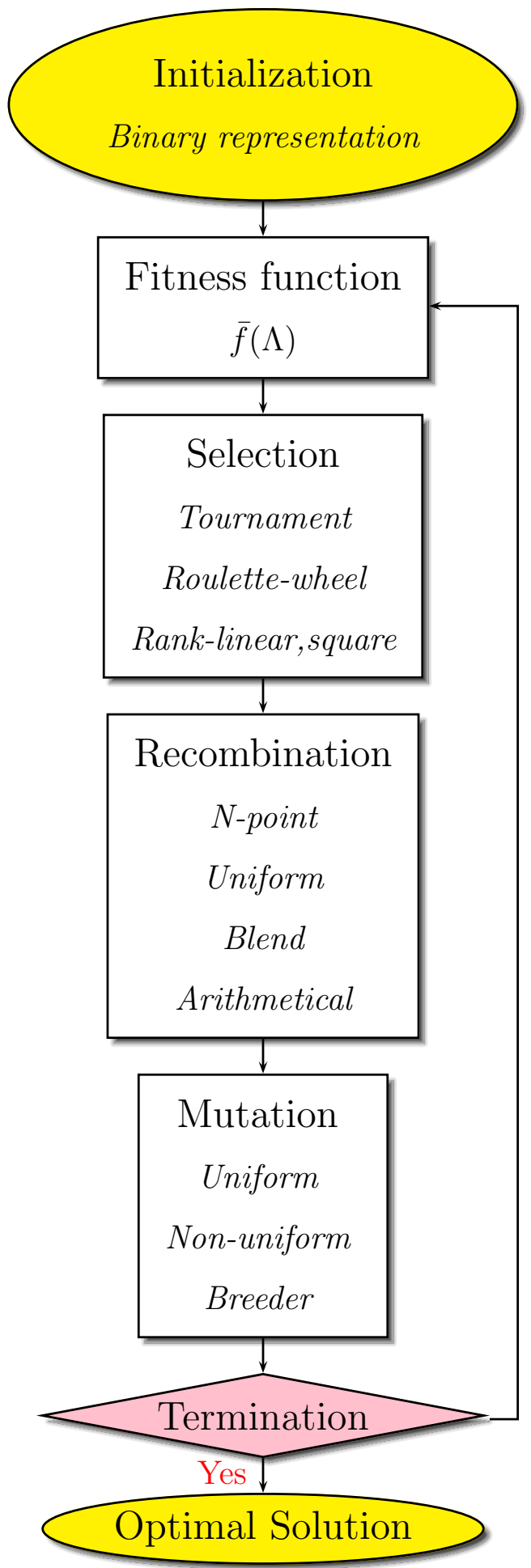




\section{Appendix F}

\section{Measurement of cellular deformation}

Cellular deformation of the entire specimen is characterized by a parameter $\varepsilon_{l}$ indicating the degree of collapse of individual cell. The degree of collapse is calculated by finding the change of cell area with respected to its original area and given as

$$
\varepsilon_{l}=1-A_{n} / A_{0}
$$

To map the collapse of whole cellular domain, a grey scale is assigned where ' 1 ' represents the undeformed state while '0' represents fully collapsed cell. 


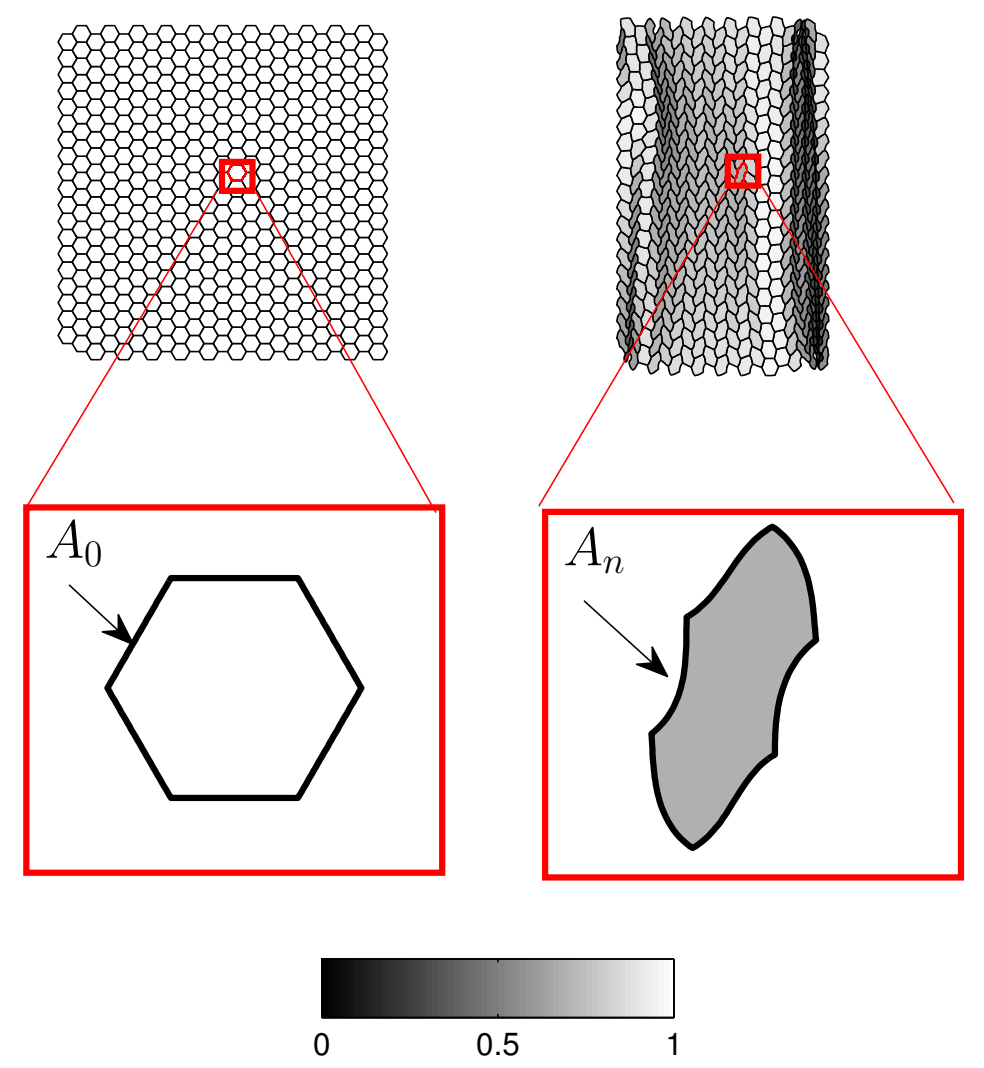

Figure F.1: Zoomed undeformed and deformed shape of a cell. 


\section{Appendix G}

\section{Copyrights}

\section{Copyright permission for Chapter 4}

This is a License Agreement between Siladitya Pal ("You") and Elsevier ("Elsevier") provided by Copyright Clearance Center ("CCC"). The license consists of your order details, the terms and conditions provided by Elsevier, and the payment terms and conditions. All payments must be made in full to CCC. For payment instructions, please see information listed at the bottom of this form.

$\begin{array}{ll}\text { Supplier } & \text { Elsevier Limited } \\ & \text { The Boulevard,Langford Lane } \\ & \text { Kidlington,Oxford,OX5 1GB,UK } \\ \text { Registered Company Number } & 1982084 \\ \text { Customer name:: } & \text { Siladitya Pal } \\ \text { Customer address } & 2011 \text { Woodmar Drive } \\ & \text { Houghton, MI 49931 } \\ \text { License number } & 2455991198046 \\ \text { License date } & \text { Jun25,2010 } \\ \text { Licensed content publisher } & \text { Elsevier }\end{array}$


Licensed content publication

Licensed content title

Licensed content author

Licensed content date

Licensed content volume number

Licensed content issue number

Number of pages

Type of Use

Portion::

Format

Are you the author of this Elsevier article?

Will you be translating?

Order reference number

Title of your thesis/dissertation

Expected completion date

Estimated size (number of pages)

Elsevier VAT number
Mechanics of Materials

Effect of microscopic deformation

mechanisms on the dynamic

response of soft cellular materials

Siladitya Pal, Spandan Maiti

Ghatu Subhash

February2010

42

2

16

reuse in a thesis/dissertation

full article

both print and electronic

Yes

No

Modeling mechanical response

of heterogeneous materials

Jul2010

250

GB494627212

\section{Copyright permission for Section 6.3}

This is a License Agreement between Siladitya Pal ("You") and Elsevier ("Elsevier") provided by Copyright Clearance Center ("CCC"). The license consists of your order details, the terms and conditions provided by Elsevier, and the payment terms and conditions. All payments must be made in full to CCC. For payment instructions, please see information listed at the bottom of this form. 
Supplier

Registered Company Number

Customer name::

Customer address

License number

License date

Licensed content publisher

Licensed content publication

Licensed content title

Licensed content author

Licensed content date

Licensed content volume number

Licensed content issue number

Number of pages

Type of Use

Requestor type

Intended publisher of new work

Portion::

Format

Are you the author of this Elsevier article?

Will you be translating?

Order reference number

Title of your thesis/dissertation
Elsevier Limited

The Boulevard,Langford Lane

Kidlington,Oxford,OX5 1GB,UK

1982084

Siladitya Pal

2011 Woodmar Drive

Houghton, MI 49931

2470850719512

Jul16, 2010

Elsevier

Computers \& Chemical Engineering Computationally efficient black-box modeling for feasibility analysis Ipsita Banerjee, Siladitya Pal, Spandan Maiti

20February2010

$\mathrm{n} / \mathrm{a}$

$\mathrm{n} / \mathrm{a}$

1

reuse in a thesis/dissertation

Not specified

other

full article

both print and electronic

Yes

No

Modeling mechanical response of heterogeneous materials 


$\begin{array}{ll}\text { Expected completion date } & \text { Jul2010 } \\ \text { Estimated size (number of pages) } & 250 \\ \text { Elsevier VAT number } & \text { GB494627212 }\end{array}$

\section{Terms and Conditions}

\section{INTRODUCTION}

1. The publisher for this copyrighted material is Elsevier. By clicking "accept" in connection with completing this licensing transaction, you agree that the following terms and conditions apply to this transaction (along with the Billing and Payment terms and conditions established by Copyright Clearance Center, Inc. ("CCC"), at the time that you opened your Rightslink account and that are available at any time at http://myaccount.copyright.com).

\section{GENERAL INTRODUCTION}

2. Elsevier hereby grants you permission to reproduce the aforementioned material subject to the terms and conditions indicated.

3. Acknowledgement: If any part of the material to be used (for example, figures) has appeared in our publication with credit or acknowledgement to another source, permission must also be sought from that source. If such permission is not obtained then that material may not be included in your publication/copies. Suitable acknowledgement to the source must be made, either as a footnote or in a reference list at the end of your publication, as follows Reprinted from Publication title, Vol /edition number, Author(s), Title of article / title of chapter, Pages No., Copyright (Year), with permission from Elsevier 
[OR APPLICABLE SOCIETY COPYRIGHT OWNER]. Also Lancet special credit - Reprinted from The Lancet, Vol. number, Author(s), Title of article, Pages No., Copyright (Year), with permission from Elsevier.

4. Reproduction of this material is confined to the purpose and/or media for which permission is hereby given.

5. Altering/Modifying Material: Not Permitted. However figures and illustrations may be altered/adapted minimally to serve your work. Any other abbreviations, additions, deletions and/or any other alterations shall be made only with prior written authorization of Elsevier Ltd. (Please contact Elsevier at permissions@elsevier.com)

6. If the permission fee for the requested use of our material is waived in this instance, please be advised that your future requests for Elsevier materials may attract a fee.

7. Reservation of Rights: Publisher reserves all rights not specifically granted in the combination of (i) the license details provided by you and accepted in the course of this licensing transaction, (ii) these terms and conditions and (iii) CCC's Billing and Payment terms and conditions.

8. License Contingent Upon Payment: While you may exercise the rights licensed immediately upon issuance of the license at the end of the licensing process for the transaction, provided that you have disclosed complete and accurate details of your proposed use, no license is finally effective unless and until full payment is received from you (either by publisher or by CCC) as provided in CCC's Billing and Payment terms and conditions. If full payment is not received on a timely basis, then any license preliminarily granted shall be deemed automatically 
revoked and shall be void as if never granted. Further, in the event that you breach any of these terms and conditions or any of CCC's. Billing and Payment terms and conditions, the license is automatically revoked and shall be void as if never granted. Use of materials as described in a revoked license, as well as any use of the materials beyond the scope of an unrevoked license, may constitute copyright infringement and publisher reserves the right to take any and all action to protect its copyright in the material.

9. Warranties: Publisher makes no representations or warranties with respect to the licensed material.

10. Indemnity: You hereby indemnify and agree to hold harmless publisher and CCC, and their respective officers, directors, employees and agents, from and against any and all claims arising out of your use of the licensed material other than as specifically authorized pursuant to this license.

11. No Transfer of License: This license is personal to you and may not be sublicensed, assigned, or transferred by you to any other person without publisher's written permission.

12. No Amendment Except in Writing: This license may not be amended except in a writing signed by both parties (or, in the case of publisher, by CCC on publisher's behalf).

13. Objection to Contrary Terms: Publisher hereby objects to any terms contained in any purchase order, acknowledgment, check endorsement or other writing prepared by you, which terms are inconsistent with these terms and conditions or CCC's Billing and Payment terms and conditions. These terms and conditions, together with CCC's Billing and Payment terms and conditions (which 
are incorporated herein), comprise the entire agreement between you and publisher (and CCC) concerning this licensing transaction. In the event of any conflict between your obligations established by these terms and conditions and those established by CCC's Billing and Payment terms and conditions, these terms and conditions shall control.

14. Revocation: Elsevier or Copyright Clearance Center may deny the permissions described in this License at their sole discretion, for any reason or no reason, with a full refund payable to you. Notice of such denial will be made using the contact information provided by you. Failure to receive such notice will not alter or invalidate the denial. In no event will Elsevier or Copyright Clearance Center be responsible or liable for any costs, expenses or damage incurred by you as a result of a denial of your permission request, other than a refund of the amount(s) paid by you to Elsevier and/or Copyright Clearance Center for denied permissions.

\section{LIMITED LICENSE}

15. Translation: This permission is granted for non-exclusive world English rights only unless your license was granted for translation rights. If you licensed translation rights you may only translate this content into the languages you requested. A professional translator must perform all translations and reproduce the content word for word preserving the integrity of the article. If this license is to re-use 1 or 2 figures then permission is granted for non-exclusive world rights in all languages.

16. Website: The following terms and conditions apply to electronic reserve and author websites:

17. Electronic reserve: If licensed material is to be posted to website, the web 
site is to be password-protected and made available only to bona fide students registered on a relevant course if: This license was made in connection with a course, This permission is granted for 1 year only. You may obtain a license for future website posting, All content posted to the web site must maintain the copyright information line on the bottom of each image, A hyper-text must be included to the Homepage of the journal from which you are licensing at http://www.sciencedirect.com/science/journal/xxxxx or the Elsevier homepage for books at http://www.elsevier.com, and Central Storage: This license does not include permission for a scanned version of the material to be stored in a central repository such as that provided by Heron/XanEdu.

18. Author website for journals with the following additional clauses: All content posted to the web site must maintain the copyright information line on the bottom of each image, and he permission granted is limited to the personal version of your paper. You are not allowed to download and post the published electronic version of your article (whether PDF or HTML, proof or final version), nor may you scan the printed edition to create an electronic version, A hyper-text must be included to the Homepage of the journal from which you are licensing at http://www.sciencedirect.com/science/journal/xxxxx , As part of our normal production process, you will receive an e-mail notice when your article appears on Elseviers online service ScienceDirect (www.sciencedirect.com). That e-mail will include the articles Digital Object Identifier (DOI). This number provides the electronic link to the published article and should be included in the posting of your personal version. We ask that you wait until you receive this e-mail and have the DOI to do any posting. Central Storage: This license does not include permission for a scanned version of the material to be stored in a central repository such as that provided by Heron/XanEdu.

19. Author website for books with the following additional clauses: Authors are 
permitted to place a brief summary of their work online only. A hyper-text must be included to the Elsevier homepage at http://www.elsevier.com All content posted to the web site must maintain the copyright information line on the bottom of each image You are not allowed to download and post the published electronic version of your chapter, nor may you scan the printed edition to create an electronic version. Central Storage: This license does not include permission for a scanned version of the material to be stored in a central repository such as that provided by Heron/XanEdu.

20. Website (regular and for author): A hyper-text must be included to the Homepage of the journal from which you are licensing at http://www.sciencedirect.com /science/journal/xxxxx. or for books to the Elsevier homepage at http://www.elsevier.com

21. Thesis/Dissertation: If your license is for use in a thesis/dissertation your thesis may be submitted to your institution in either print or electronic form. Should your thesis be published commercially, please reapply for permission. These requirements include permission for the Library and Archives of Canada to supply single copies, on demand, of the complete thesis and include permission for UMI to supply single copies, on demand, of the complete thesis. Should your thesis be published commercially, please reapply for permission.

22. Gratis licenses (referencing 0 in the Total field) are free. Please retain this printable license for your reference. No payment is required. If you would like to pay for this license now, please remit this license along with your payment made payable to "COPYRIGHT CLEARANCE CENTER" otherwise you will be invoiced within 48 hours of the license date. Payment should be in the form of a check or money order referencing your account number and this invoice number RLNK10805893. Once you receive your invoice for this order, 
you may pay your invoice by credit card. Please follow instructions provided at that time. Make Payment To: Copyright Clearance Center Dept 001 P.O. Box 843006 Boston, MA 02284-3006 If you find copyrighted material related to this license will not be used and wish to cancel, please contact us referencing this license number 2455991198046 and noting the reason for cancellation. Questions? customercare@copyright.com or +1-877-622-5543 (toll free in the US) or $+1-978-646-2777$. 


\section{References}

Aboudi, J., 1982. A continuum theory for fiber reinforced elastic-viscoplastic composites. Journal of Engineering Science 20 (55), 605-621.

Aldred, N., Wills, T., Williams, D. N., Clare, A. S., 2007. Tensile and dynamic mechanical analysis of the distal portion of mussel (mytilus edulis) byssal threads. J. R. Soc. Interface 4, 1159-1167.

Alper, J., 2002. Stretching the limits. Science 297, 329-331.

Andrade, J. E., Tu, X., 2009. Multiscale framework for behavior prediction in granular media. Mechanics of Materials 41, 652-669.

Avalle, M., Belingardi, G., Montanini, R., 2001. Characterization of polymeric structural foams under compressive impact loading by means of energy-absorption diagram. International Journal of Impact Engineering 25, 445-472.

Bahei-El-Din, Y., Rajendran, A., Zikry, M., 2004. A micromechanical model for damage progression in woven composite system. International Journal of Solids and Structures 41, 2307-2330.

Banerjee, I., Pal, S., Maiti, S., 2010. Computationally efficient black-box modeling for feasibility analysis. Computers \& Chemical Engineering Article in press.

Bell, E., Gosline, J. M., 1996. Mechanical design of mussel byssus: material yield 
enhances attachment strength. The Journal of Experimental Biology 199, 10051017.

Bell, E. C., Gosline, J. M., 1997. Strategies for life in flow: tenacity, morphometry, and probability of dislodgment of two mytilus species. Marine Ecology and Progress Series 159, 197-208.

Belytschko, T., Chiapetta, R., Bartel, H., 1976. Efficient large scale non-linear transient analysis by finite elements. International Journal for Numerical Methods in Engineering 10, 579-596.

Belytschko, T., Hsieh, B. J., 1973. Nonlinear transient finite element analysis with convected coordinates. International Journal for Numerical Methods in Engineering $7,255-271$.

Belytschko, T., Liu, W., Moran, B., 2000. Nonlinear finite elements for continua and structures. John Wiley \& Sons, Ltd., New York, USA.

Bertoldi, K., Boyce, M. C., 2007. Mechanics of the hysteretic large strain behavior of mussel byssus thread. Journal of Material Science 42, 8943-8956.

Beryland, L., Kolpakov, A., 2001. Network approximation in the limit of small interparticle distance of the effective properties of a high contrast random dispersed composite. Arch. Rational Mech. Anal 159, 179-227.

Bonet, J., Wood, R., 2008. Nonlinear continuum mechanics for finite element analyis. Cambridge University Press.

Bouix, R., Viot, P., Lataillade, J. L., 2009. Polypropylene foam behavior under dynamic loadings: Strain rate, density and microstructure effects. International Journal of Impact Engineering 36, 329-342.

Braze, S. L., Carrington, E., 2006. Interspecific comparison of the mechanical properties of mussel byssus. Biological Bulletine 211, 263-274. 
Brezny, R., Green, D. J., 1990. The effect of cell size on the mechanical behavior of cellular materials. Acta Metallurgica et Materialia 38 (12), 2517-2526.

Buryanchenko, V., 2007. Micromechanics of Heterogeneous Materials. Springer.

Calladine, C. R., English, R. W., 1984. Strain-rate and inertia effects in the collapse of two types of energy-absorbing structure. International Journal of Mechanical Sciences 26, 689-701.

Carrington, E., Gosline, J. M., 2004. Mechanical design of mussel byssus: load cycle and strain rate dependence. American Malacological Bulletin 18 (1-2), 135-142.

Carter, D. R., Hayes, W. C., 1976. The compressive behavior of bone as a two-phase porous structure. The Journal of Bone and Joint Surgery 59 (7), 954-962.

Castaneda, P., 1989. The overall constitutive behavior of nonlinearly elastic composites. Proc. R. Soc. London Ser. A 422, 147-171.

Castaneda, P., 1996. Exact second order estimates for the effective mechanical properties of nonlinear composite material. Journal of the Mechanics and Physics of Solids 44, 827-862.

Castaneda, P., Suquet, P., 1998. Nonlinear composites. Adv. Appl. Mech. 34, 171-302.

Castaneda, P., Tiberio, E., 2000. A second-order homogenization method in finite elasticity and applications to black-filled elastomers. Journal of the Mechanics and Physics of Solids 48, 1389-1411.

Chakravarty, U., Mahfuz, H., Saha, M., Jeelani, S., 2003. Strain rate effects on sandwich core materials: An experimental and analytical investigation. Acta Materialia 51, 1469-1479.

Chen, W., Lu, F., Frew, D., Forrestal, M., 2002a. Dynamic compression testing of soft materials. Trans. of the ASME 69, 214-223. 
Chen, W., Lu, F., Frew, D. J., Forrestal, M. J., 2002b. Dynamic compression testing of soft materials. Transaction of the ASME 69, 214-223.

Chen, W., Lu, F., Winfree, N., 2002c. High strain-rate compressive behavior of a rigid polyurethane foam with various densities. Experimental Mechanics 42 (1), 65-73.

Chen, W., Lu, F., Winfree, N., 2002d. High strain rate compressive behavior of a rigid polyurethane foam with various densities. Experimental Mechanics 42 (1), 65-73.

Chen, W., Ravichandran, G., 2000. Failure mode transition in ceramics under dynamic multiaxial compression. International Journal of Fracture 101, 141-159.

Chen, W., Wu, Q., Kang, J., Winfree, N., 2001. Compressive superelastic behavior of a NiTi Shape memory alloy at strain rates of 0.001 to $750 /$ s. International Journal of Solids and Structures 38, 8989-8998.

Chen, W., Zhang, B., Forrestal, M., 1999. A split Hopkinson bar technique for lowimpedance materials. Experimental Mechanics 39 (2), 81-85.

Chung, J., Waas, A. M., 2002a. Compressive response of cellular cell polycarbonate honeycomb under inplane biaxial static and dynamic loading. Part I: experiments. International Journal of Impact Engineering 27, 729-754.

Chung, J., Waas, A. M., 2002b. Compressive response of cellular cell polycarbonate honeycomb under inplane biaxial static and dynamic loading. Part II: simulations. International Journal of Impact Engineering 27, 1015-1047.

Cook, R., Malkus, D., Plesha, M., Witt, R., 2002. Concepts and applications of finite element analysis. John Wiley \& Sons, Ltd., New York, USA.

Coyne, K. J., Qin, X. X., Waite, J. H., 1997. Extensible collagen in mussel byssus: a natural block copolymer. Science 277, 1830-1832.

Crisfield, M., 1991. Non-linear finite element analysis of solids and structures. Vol. 1. John Wiley \& Sons, Ltd., New York, USA. 
Daxner, T., Bohm, H. J., Rammerstorfer, F. G., 1999. Mesoscopic simulation of inhomogeneous metallic foams with respect to energy absorption. Computational Material Science 16, 61-69.

Denny, M. W., 1988. Biology and mechanics of the wave swept environment. Princeton University Press, Princeton, NJ.

Deshpande, V. S., Fleck, N. A., 2000. High strain rate compressive behavior of aluminum. International Journal of Impact Engineering 24, 277-298.

Dvorak, G., 1992. Transformation field analysis of inelastic composite materials. Proc. R. Soc. London A 437, 311-327.

Elnasri, I., Pattofatto, S., Zhao, H., Tsitsiris, H., Hild, F., Girard, Y., 2007. Shock enhancement of cellular sreuctures under impact loading: Part i experiment. Journal of Mechanics and Physics of Solids 55, 2652-2671.

Espinosa, H., Rim, J., Barthelat, F., Buehler, M., 2009. Merger of structure and material in nacre and bone- perspective on de novo biomimetic materials. Progress in Material Science 54, 1059-1100.

Feyel, F., 2003. A multilevel finite element method (fe2) to describe the response of highly non-linear structures using generalized continua. Comput. Methods Appl. Mech. Engrg 192, 3233-3244.

Feyel, F., Chaboche, J., 2000. Fe2 multiscale approach for modelling the elastoviscoplastic behavior of long fiber sic/ti composite materials. Comput. Methods Appl. Mech. Engrg 183, 309-330.

Fish, J., Shek, K., 1999. Finite deformation plasticity of composite structures: computational models and adaptive strategies. Comput. Methods Appl. Mech. Engrg. 172, 145-174. 
Fish, J., Shek, K., Shepahrd, M., Pandheeradi, M., 1997. Computational plasticity for composite structure based on mathematical homogenization:theory and practice. Comput. Methods Appl. Mech. Engrg. 148, 53-73.

Fish, J., Yu, Q., Shek, K., 1999. Computational damage mechanics for composite material based on mathematical homogenization. International Journal of Numerical Methods in Engineering 45, 1657-1679.

Forrestal, M. J., Wright, T. W., Chen, W., 2007. The effect of radial inertia on brittle samples during the split hopkinson pressure bar test. International Journal of Impact Engineering 34, 405-411.

Frew, D. J., Forrestal, M. J., Chen, W., 2002. Pulse shaping techniques for testing brittle materials with a split hopkinson pressure bar. Experimental Mechanics 42, 93-106.

Frew, D. J., Forrestal, M. J., Chen, W., 2005. Pulse shaping techniques for testing elastic-plastic materials with a split Hopkinson pressure bar. Experimental Mechanics 45 (2), 186-195.

Frew, D. J., Forrestal, M. J., Chen, W., 2006. Pulse shaping techniques for testing brittle materials a split hopkinson pressure bar. Experimental Mechanics 42 (1), 93-106.

Gao, Z., Yu, T., Lu, G., 2005. A study on type ii structures. part i: a modified onedimensional mass-spring model. International Journal of Impact Engineering 31, 895-910.

Gary, G., Bailly, P., 1998. Behavior of quasi-brittle material at high strain rate. experiment and modelling. European Journal of Mechanics A/Solids 17 (3), 403420. 
Ghosh, S., Lee, K., Raghavan, P., 2001. A multi-level computational model for multiscale damage analysis in composite and porous material. International Journal of Solids and Structure 38, 2335-2385.

Ghosh, S., Moorthy, S., 1995. Elastic-plastic analysis of arbitary heterogeneous materials with the voronoi cell finite element method. Comput. Methods Appl. Mech. Engrg. 121, 373-409.

Gibson, L., Ashby, M., 1997. Cellular solids: Structure and properties. Cambridge University Press, Cambridge, UK.

Gosline, J., Lillie, M., Carrington, E., Guerette, P., Ortelepp, C., Savage, K., 2002. Elastic proteins: biological roles and mechanical properties. Phil. Trans. R. Soc. Lond B 357, 121-132.

Gray, G., 2000. Classic split Hopkinson pressure bar testing. Mechanical Testing and Evaluation, American Society for Metals 8, 462-472.

Guedes, J. M., Kikuchi, N., 1990. Prepocessing and postprocessing for materials based on the homogenization method with adaptive finite element method. Comput. Methods Appl. Mech. Engrg. 83, 143-198.

Hagenau, A., Hcheidt, H. A., Serpell, L., Huster, D., Scheibel, T., 2008. Structural analysis of proteinaceous components in byssal threads of the mussel mytilus galloprovincialis. Macromolecular Bioscience 8, 1-7.

Halemane, K., Grossmann, I., 1983. Optimal process design under uncertainty. AIChE Journal 29, 425.

Harrigan, J. J., Reid, S. R., Peng, C., 1999. Inertia effects in impact energy absorbing materials and structures. International Journal of Impact Engineering 22, 955-979. 
Harrington, M. J., Waite, J. H., 2007. Holdfast heroics: comparing the molecular and mechanical properties of mytilus californianus byssal threads. The Journal of Experimental Biology 201, 4307-4318.

Harrington, M. J., Waite, J. H., 2009. How nature modulates a fiber's mechanical properties: Mechanically distinct fibers drawn from natural mesogenic block copolymer variants. Advanced Materials 21, 440-444.

Hasin, Z., 1985. Large isotropic elastic deformations and porous media. International Journal of Solids and Structures 21, 711-720.

Hearle, J. W. S., 2007. Protein fibers: structural mechanics and future opportunities. Journal of Material Science 42, 8010-8019.

Hill, R., 1972. On constitutive macro-variables for heterogeneous solids at finite strain. Proceedings of the Royal Society of London. Series A, Mathematical and Physical Sciences 326 (1565), 131-147.

Hilyard, N., 1982. Mechanics of Cellular Plastics. Applied Science Publishers Ltd., London, UK.

Hönig, A., Stronge, W. J., 2002a. In-plane dynamic crushing of honeycomb. Part I: crush band initiation and wave trapping. International Journal of Mechanical Sciences 44 (8), 1665-96.

Hönig, A., Stronge, W. J., 2002b. In-plane dynamic crushing of honeycomb. Part II: application to impact. International Journal of Mechanical Sciences 44 (8), 1697-96.

Juntikka, R., Hallstron, S., 2004. Weight-balanced drop test method for characterization of dynamic properties of cellular materials. International Journal of Impact Engineering 30, 541-554.

Kanoute, P., Boso, D., Chaboche, J., Schrefler, B., 2009. Multiscale methods for composites: a review. Arch Comput Methods Eng 16, 31-75. 
Karagiozova, D., Jones, N., 1996. Dynamic elastic-plastic buckling phenomena in a rod due to axial impact. International Journal of Impact Engineering 18 (7-8), 919-947.

Klepaczko, J. R., 1988. On the role of microcracking inertia in rate sensitivity of coal at high strain rates. Archieves of Mechanics 40, 345-358.

Kolsky, H., 1949. An investigation of the mechanical properties of materials at very high strain rates of loading. Proceedings of the Royal Society of London B62, 676700.

Kouznetsova, V., Geers, M., Brekelmans, W., 2004. Multi-scale second-order computational homogenization of multi-phase materials: a nested finite element solution strategy. Comput. Methods Appl. Mech. Engrg 193, 5525-5550.

Krysl, P., Lall, S., Marsden, J., 2001. Dimensional model reduction in non-linear finite element dynamics of solids and structures. International Journal of Numerical Methods in Engineering 51, 479-504.

Lall, S., Krysl, P., Marsden, J., 2003. Structure preserving model reduction for mechanical systems. Physica D 184, 304-318.

Langseth, M., Hopperstad, O., 1996. Static and dynamic axial crushing of thin walled aluminum extrusions. International Journal of Impact Engineering 18, 949-968.

Langseth, M., Hopperstad, O., Berstad, T., 1999. Crashworthiness of aluminium extrusions: validation of numerical simulation, effect of mass ratio and impact velocity. International Journal of Impact Engineering 22, 829-854.

Lee, S., Barthelat, F., Moldovan, N., Espinosa, H. D., Wadley, H. N., 2006. Deformation rate effects on failure modes of open cell al foams and textile cellular materials. International Journal of Solids and Structures 43, 53-73. 
Li, K., Gao, X.-L., Wang, J., 2007. Dynamic crushing behavior of honeycomb structures with irregular cell shapes and non-uniform cell wall thickness. International Journal of Solids and Structures 44, 5003-5026.

Li, Q. M., Meng, H., 2003. About the dynamic strength enhancement of concrete-like materials in a split hopkinson pressure bar test. International Journal of Solids and Structures 40, 343-360.

Lin, Q., Gourdon, D., Sun, C., Andersen, N., Anderson, T. H., Waite, J. H., Israelachvili, J. N., 2007. Adhesion mechanisms of the mussel foot proteins mfp-1 and mfp-3. Procedings of National Academy of Science 104 (10), 3782-3786.

Liu, Q., Subhash, G., 2006. Characterization of viscoelastic properties of polymer bar using iterative deconvolution in the time domain. Mechanics of Materials 38 (12), $1105-1117$.

Lopez-Pamies, O., Castaneda, P., 2004. Second-order estimates for the macroscopic response and loss of ellipticity in porous rubber at large deformation. Journal of Elasticity 76, 247-287.

Lucas, J. M., Vaccaro, E., Waite, J. H., 2002. A molecular, morphometric and mechanical comparison of the structural element of byssus from mytilus edulis and mytilus galloprovincialis. The Journal of Experimental Biology 205, 1807-1817.

Ma, G. W., Ye, Z. Q., Shao, Z. S., 2009. Modeling loading rate effect on crushing stress of metallic cellular materials. International Journal of Impact Engineering doi:101016/j.ijimpend.2008.11.013, 1-8.

McElhaney, J. H., 1966. Dynamic response of bone and muscle tissue. Journal of Applied Physiology 21 (4), 1231-1236.

McVeigh, C., Vernerey, F., Liu, W. K., Brinson, L. C., 2006. Multiresolution analysis for material design. Comput. Methods Appl. Mech. Engrg 195, 5053-5076. 
Miche, C., 2003. Computational micro-to-macro transitions for discretized microstructures of heterogeneous materials at finite strains based on the minimization of averaged incremental energy. Comput. Methods Appl. Mech. Engrg 192, 559-591.

Miche, C., Schröder, J., Schotte, J., 1999. Computational homogenization analysis in finite plasticity simulation of texture development in polycrystalline materials. Comput. Methods Appl. Mech. Engrg. 171 (3-4), 387-418.

Michel, J., Moulinec, H., Suquet, P., 1999. Effective properties of coposite materials with periodic microstructure: a computational approach. Comput. Methods Appl. Mech. Engrg 172, 109-143.

Moeser, G. M., Carrington, E., 2006. Seasonal influence of wave action on thread production in mytilus edulis. The Journal of Experimental Biology 206, 1996-2003.

Moulinec, H., Suquet, P., 1994. A fast numerical method for computing the linear and nonlinear properties of composites. C.R. Acad. Sci Paris II 318, 1417-1423.

Moulinec, H., Suquet, P., 1998. A numerical method for coputing the overall response of nonlinear composites with complex microstrcuture. Comput. Methods Appl. Mech. Engrg 157, 69-94.

Mousavi, S., Welch, K., Valdek, U., Lundberg, B., 2005. Non-equilibrium split hopkinson pressure bar procedure for non-parametric identification of complex modlus. International Journal of Impact Engineering 31, 1133-1151.

Muller, S., 1987. Homogenization of nonconvex integral functionals and cellular elastic materials. Arch. Rational Mech. Anal 99, 189-212.

Nemat-Nasser, S., Hori, M., 1993. Micromechanics: overall properties of heterogeneous materials, North-Holland series in Applied Mathematics and Mechanics. Vol. 37. North-Holland, Amsterdam. 
Nemat-Nasser, S., Kang, W. J., McGee, J. D., Guo, W.-G., Issacs, J. B., 2007. Experimental investigation of energy absorption characteristics of components of sandwich structures. International Journal of Impact Engineering 34, 1119-1146.

Nie, X., Song, B., Ge, Y., Chen, W., Weerasooriya, T., 2009. Dynamic tensile tesing of soft materials. Experimental Mechanics 49, 451-458.

Niroomandi, S., Alfaro, I., Cueto, E., Chinesta, F., 2010. Model order reduction for hyperelastic materials. International Journal of Numerical Methods in Engineering $81,1180-1206$

Nittur, P. G., Maiti, S., Geubelle, P. H., 2008. Grain-level analysis of dynamic fragmentation of ceramics under multi-axial compression. Journal of Mechanics and Physics of Solids 56, 993-1017.

Ogden, R., 1978. Extermum priciples in nonlinear elastic and their application to composites- i, theory. International Journal of Solids and Structures 14, 265-282.

Oskay, C., 2009. Two-level multiscale enrichment methodology for modeling heterogeneous plates. International Journal of Numerical Methods in Engineering 80, $1143-1170$.

Oskay, C., Fish, J., 2007. Eigendeformation based reduced order homogenization for failure analysis of heterogeneous materials. Comput. Methods Appl. Mech. Engrg. $196,1216-1243$.

Pal, S., Maiti, S., Subhash, G., 2010. Effect of microscopic deformation mechanisms on the dynamic response of soft cellular materials. Mechanics of Materials 42, 118133.

Papov, V. V., Diamond, T. V., Biemann, K., Waite, J. H., 1995. Hydroxyargininecontaining polyphenolic proteins in the adhesive plaques of the marine mussel mytilus edulis. The Journal of Biological Chemistry 270, 20183-20192. 
Pattofatto, S., Elnasri, I., Zhao, H., Tsitsiris, H., Hild, F., Girard, Y., 2007. Shock enhancement of cellular sreuctures under impact loading: Part i experiment. Journal of Mechanics and Physics of Solids 55, 2672-2686.

Qin, X. X., Coyne, K. J., Waite, J. H., 1997. Tough tendons: mussel byssus has collagen with silk-like domain. The Journal of Biological Chemistry 272 (51), 3262332627.

Rabitz, H., Alis, O., 1999. General foundations of high dimensional model representations. Journal of Mathematical Chemistry 25, 197.

Rabitz, H., Alis, O., Shorter, J., Shim, K., 1998. Efficient input-output model representations. Computer Physics Communications 115, 1.

Ravichandran, G., Subhash, G., 1994. Critical appraisal of limiting strain rates for compression testing of ceramics in a split Hopkinson pressure bar. Journal of American Ceramic Society 77 (1), 263-267.

Reid, S. R., Peng, C., 1997. Dynamic uniaxial crushing of wood. International Journal of Impact Engineeing 19 (5-6), 531-570.

Romero, P. A., Zheng, S. F., Cuitino, A. M., 2008. Modeling the dynamic response of visco-elastic open-cell foams. Journal of the Mechanics and Physics of Solids 56, 1916-1943.

Rosakis, P., Ruina, A., Lakes, R. S., 1993. Microbuckling instability in elastomeric cellular solids. Journal of Material Science 28, 4667-4672.

Ross, C., Jerome, D., Tedesco, J., Hughes, M., 1996. Moisture and strain rates effects on concrete strength. ACI Mater. J. 93, 293-300.

Roux, W., Stander, N., Haftka, R., 1998. Response surface approximations for structural optimization. Int. J. Numer. Meth. Engrg. 42, 517. 
Ruan, D., Lu, G., Wang, B., Yu, T. X., 2003. Inplane dynamic crushing of honeycombs-a finite element study. International Journal of Impact Engineering $28,161-182$.

Ryckelynck, D., 2009. Hyper-reduction of mechanical models involving internal variables. International Journal of Numerical Methods in Engineering 77, 75-89.

Rzepecki, L. M., Hansen, K. M., Waite, J. H., 1992. Characterization of a cystinerich polyphenolic protein family from the blue mussel mytilus edulis l. Biological Bulletin 183, 123-137.

Saha, M. C., Mahfuz, H., Chakravarty, U. K., Uddin, U., Kabir, M. E., Jeelani, S., 2005. Effect of density, microstructure, and strain rate on compression behavior of polymeric foams. Material Science and Engineering A 406, 328-336.

Scheibel, T., 2005. Protein fibers as performance proteins: new technologies and applications. Current Opinion in Biotechnology 16, 427-433.

Shergold, O., Fleck, N., Radford, D., 2006. The uniaxial stress versus strain response of pig skin and silicone rubber at low and high strain rates. International Journal of Impact Engineering 32, 1384-1402.

Shim, J., Mohr, D., 2008. Using split Hopkinson pressure bars to perform large strain compression tests on polyure. International Journal of Impact Engineering doi:10.1016/j.ijimpeng.2008.12.010, 1-12.

Silverman, H. G., Roberto, F. F., 2007. Understanding the marine mussel adhesion. Marine biotechnology 9, 661-681.

Smit, R., Brekelmans, W., Meijer, H., 1998. Prediction of the mechanical behavior of nonlinearly heterogeneous systems by multi-level finite element modeling. Comput. Methods Appl. Mech. Engrg. 155, 181-192.

Smith, B., 2003. The boeing 777. Advanced Materials \& Processes 43, 41-44. 
Song, B., Chen, W., 2003. Dynamic compressive constitutive behavior of EPDM rubber. Trans of ASME, Journal of Engineering Materials and Technology 125, 294-301.

Song, B., Chen, W., 2004. Dynamic stress equilibration in split Hopkinson pressure bar tests on soft materials. Experimental Mechanics 44 (3), 300-312.

Song, B., Chen, W., Weerasooriya, T., 2003. Quasistatic and dynamic compressive behavior of a S-2 Glass/SC15 composite. Journal of Composite Materials 37, 17231743.

Song, B., Ge, Y., Chen, W. W., Weerasooriya, T., 2007. Radial inertia effects in kolsky bar testing of extra-soft specimen. Experimental Mechanics 47, 659-670.

Song, B., Syn, C. J., Grupido, C. L., Chen, W., Lu, W. Y., 2008. A long spli hopkinson pressure bar (lshpb) for intermediate-rate characterization of soft materials. Experimental Mechanics 48, 809-815.

Sparks, J. L., Dupaix, R. B., 2008. Constitutive modeling of rate-dependent stressstrain behavior of human liver in blunt impact loading. Annals of Biomedical Engineering 36 (11), 1883-1892.

Su, X., Yu, T., Reid, S., 1995. Inertia-sensitive impact energy absorbing structures part i: Effects of inertia and elasticity. International Journal of Impact Engineering $16(4), 651-672$.

Subhash, G., Liu, Q., 2004. Crushability maps for structural polymeric foams in uniaxial loading under rigid confinement. Experimental Mechanics 44, 289-294.

Subhash, G., Liu, Q., Gao, X.-L., 2006a. Quasistatic and high strain rate uniaxial compressive response of polymeric structural foam. International Journal of Impact Engineering 32, 1113-1126. 
Subhash, G., Liu, Q., Gao, X. L., 2006b. Quasistatic and high strain rate uniaxial compressive response of polymeric structural foam. International Journal of Impact Engineering 32, 1113-1126.

Subhash, G., Ravichandran, G., 2000a. Split Hopkinson pressure bar testing of ceramics. Mechanical Testing and Evaluation, American Society for Metals 8, 497-504.

Subhash, G., Ravichandran, G., 2000b. Split Hopkinson pressure bar testing of ceramics. Mechanical Testing and Evaluation, American Society for Metals 8, 497-504.

Tagarielli, V. L., Deshpande, V. S., Fleck, N. A., 2008. The high strain rate response of PVC foams and end-grain balsa wood. Composite Part B 39, 83-91.

Tam, L. L., Calladine, C. R., 1991. Inertia and strain rate effects in a simple platestructure under impact loading. International Journal of Impact Engineeing 11 (3), 349-377.

Tan, P. J., Harrigan, J. J., Reid, S. R., 2002. Inertia effects in uniaxial dynamic compression of a closed cell aluminum alloy foam. Materials Science and Technology $18(5), 480-488$.

Tan, P. J., Reid, S. R., Harrigan, J. J., ans S. Li, Z. Z., 2005. Dynamic compressive strength properties of aluminum foams. part ii-'shock'theory and comparison with experimental data and numerical models. Journal of the Mechanics and Physics of Solids 53, 2206-2230.

Tedesco, J. W., Ross, C. A., Kuennen, S. T., 1993. Strain rate effects on the compressive strength of shock mitigating foams. Journal of Sounds and Vibrations 165 (2), $376-384$.

Terada, K., Kikuchi, N., 2001. A class of general algorithms for multi-scale analyses of heterogeneous media. Comput. Methods Appl. Mech. Engrg 190, 5427-5464. 
Thomas, T., Mahfuz, H., Carlsson, L., Kanny, K., Jeelani, S., 2002. Dynamic compression of cellular cores: temperature and strain rate effects. Composite Structures $58,505-512$.

Thomas, T., Mahfuz, H., Kanny, K., 2004. Dynamic compression of sandwich composites at sub-ambient temperatures. Journal of Composite Materials 38 (8), 641-653.

Torquato, S., 2001. Random Heterogeneous materials. Springer.

Troncoso, O. P., Torres, F. G., Grande, C. J., 2008. Characterization of the mechanical properties of tough biopolymer fibers from the mussel byssus of aulacomya ater. Acta Biomaterialia.

Vaccaro, E., Waite, J. H., 2001. Yield and post-yield behavior of mussel byssal thread: a self-healing biomolecular material. Biomacromolecules 2, 906-911.

van Hest, J. C. M., Tirrel, D. A., 2001. Protein based materials, towards a new level of structural control. Chemical communication, 1897-1904.

Vecchio, K., Jiang, F., 2007. Improved pulse shaping to achieve constant strain rate and stress equlibrium in split Hopkinson pressure bar testing. Metallurgical and Materials Transactions A 38a, 2655-2665.

Viot, P., Shankar, K., Bernard, D., 2008. Effect of strain rate and density on dynamic behavior of syntactic foam. Composite Structures 86, 314-327.

Voit, P., Bernard, D., Plougonven, E., 2007. Polymeric foam deformation under dynamic loading by the use of the microtomographic technique. Journal of Material Science 42, 7202-7213.

Vreeland, V., Waite, J. H., Epstein, L., 1998. Polyphenols and oxidases in substratum adhesion by marine algae and mussel. Journal of Phycology 34, 1-8. 
Vural, M., Ravichandran, G., 2003. Dynamic response and energy dissipation characteristics of balsa wood: experiment and analysis. International Journal of Solids and Structures 40, 2147-2170.

Waite, J. H., Andersen, N. H., Jewhurst, S., Sun, C., 2005. Mussel adhesion: finding the tricks worth mimicking. The Journal of Adhesion 81, 297-317.

Waite, J. H., Qin, X., 2001. Polyphosphoprotein from the adhesive pads of mytilus edulis. Biochemistry 40, 2887-2893.

Waite, J. H., Vaccaro, E., Sun, C., Lucas, J. M., 2002. Elastomeric gradients: a hedge against stress concentration in marine holdfast. Phil. Trans. R. Soc. Lond B 357, $143-153$.

Warburton, W., 1976. The dynamical behavior of structures. Pergamon Press.

Warner, S. C., Waite, J. H., 1999. Expression of multiple forms of an adhesive plaque protein in an individual mussel, mytilus edulis. Marine Biology 134, 729-734.

Weickum, G., Eldred, M., Maute, K., 2009. A multi-point reduced-order modeling approach of transient strcutural dynamics with application to robust design optimization. Struct. Multidisc. Optim 38, 599-611.

Wiegemann, M., 2005. Adhesion in blue mussel (mytilus edulis) and barnacles (genus balanus): Mechanisms and technical applications. Aquatic Science 67, 166-176.

Wills, J., 1981. Variational and related methods for the overall properties of composites,Advances in Applied Mechanics. Vol. 21. Academic Press.

Wu, E., Jiang, W., 1997. Axial crush of metallic honeycombs. International Journal of Impact Engineering 19 (5-6), 439-456.

Yang, L. M., Shim, V. P. W., 2005. An analysis of stress uniformity in split Hopkinson bar test specimen. International Journal of Impact Engineering 31, 129-150. 
Yi, F., Zhu, Z., Zu, F., Hu, S., Pan, Y., 2001. Strain rate effects on the compressive properties and the energy-absorbing capacity of aluminum alloy foams. Materials Characterization 47, 417-422.

Yvonnet, J., Gonzalez, D., He, Q., 2009. Numerically explicit potential for the homogenization of nonlinear elastic heterogeneous materials. Comput. Methods Appl. Mech. Engrg. 198, 2723.

Yvonnet, J., He, Q., 2007. The reduced model multiscale method (r3m) for non-linear homogenization of hyperelastic media at finite strains. Journal of Computational Physics 223, 314 .

Zhao, H., Abdennadher, S., 2004. On the strength enhancement under impact loading of square tubes made from rate insensitive metals. International Journal of Solid and Structures 41, 6677-6697.

Zhao, H., Elnasri, I., Li, H., 2006. The mechanisms of strength enhancement under impact loading of cellular materials. Advanced Engineering Materials 8 (9), 877893.

Zhao, H., I.Elnasri, Abdennadher, S., 2005. An experimental study on the behavior under impact loading of metallic cellular materials. International Journal of Mechanical Science 47, 757-774.

Zheng, Z., Yu, J., Li, J., 2005a. Dynamic crushing of 2D cellular structures:. International Journal of Impact Engineering 32, 650-644.

Zheng, Z., Yu, J., Li, J., 2005b. Dynamic crushing of 2D cellular structures: A finite element study. International Journal of Impact Engineering 32, 650-664. 
